\title{
OPTICALLY DETECTED MAGNETIC RESONANCE OF MOLECULES IN EXCITED TRIPLET STATF.S
}

\author{
Michael J. Buckley \\ (Ph. D. Thesis) \\ February 1971
}

AEC Contract No. W-7405-eng-48
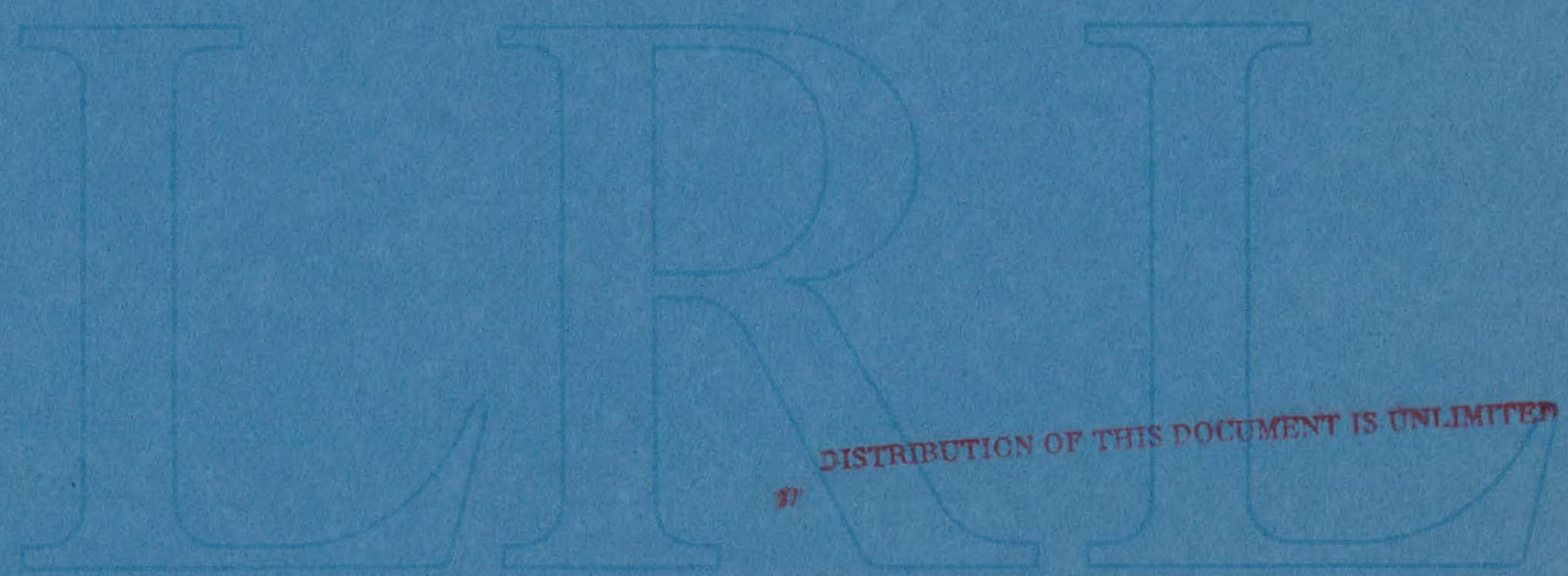

\section{LAWRENCE RADIATION LABORATORY UNIVERSITY of CALIFORNIA BERKELEY}




\section{DISCLAIMER}

This report was prepared as an account of work sponsored by an agency of the United States Government. Neither the United States Government nor any agency Thereof, nor any of their employees, makes any warranty, express or implied, or assumes any legal liability or responsibility for the accuracy, completeness, or usefulness of any information, apparatus, product, or process disclosed, or represents that its use would not infringe privately owned rights. Reference herein to any specific commercial product, process, or service by trade name, trademark, manufacturer, or otherwise does not necessarily constitute or imply its endorsement, recommendation, or favoring by the United States Government or any agency thereof. The views and opinions of authors expressed herein do not necessarily state or reflect those of the United States Government or any agency thereof. 


\section{DISCLAIMER}

Portions of this document may be illegible in electronic image products. Images are produced from the best available original document. 


\section{PAGES $\mathrm{i}$ to $\mathrm{ii}$ WERE INTENTIONALLY LEFT BLANK}


TABLE OF CONTENTS

\section{ABSTRACT}

I. Introduction

A. The Excited Triplet State in Organic Molecules..............

B. The Historical Development of ODMR ..................

II. Sensitivity Considerations in Optical Detection..............15

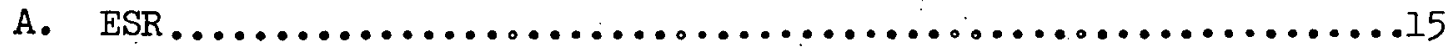

1. The Effect of the Radiative Rate Constants.............22

2. The Effect of Spin Lattice Relaxation..................25

3. The Effect of Non-Radiative Relaxation...............28

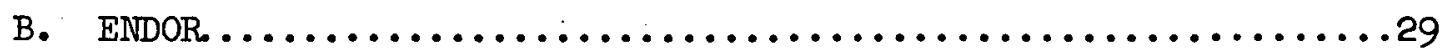

III. The Spin Hamiltonian

A. $\mathrm{H}_{\mathrm{SS}}$ - The Spin-Spin or Zero Field Splitting Hamiltonian $\cdots . . .33$

B. $\mathrm{H}_{\mathrm{Q}}$ - The Nuclear Quadmupole Hamiltonian.....................

C. $\mathrm{H}_{\mathrm{HF}}$ - The Nuclear-Electron Hyperfine Hamiltonian...........48

D. The Total Hamiltonian - Energy Levels and Transition

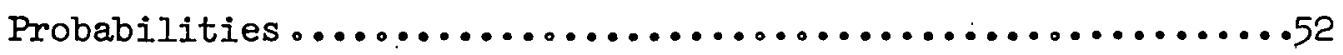

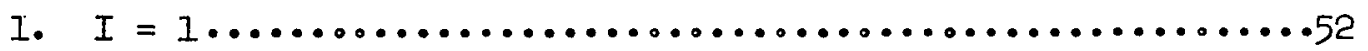

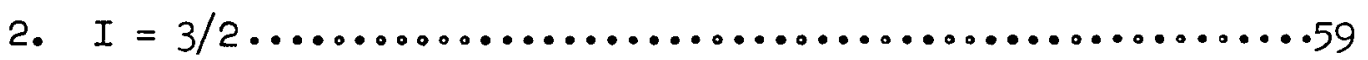

IV. Experimental Equipment and Procedure $\ldots \ldots \ldots \ldots \ldots \ldots \ldots \ldots \ldots \ldots \ldots$

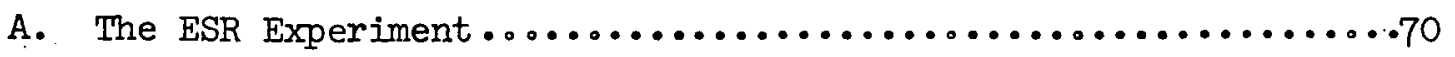

B. The ENDOR Experiment............................72

C. Variations of the Basic Experiments $\ldots \ldots \ldots \ldots \ldots \ldots \ldots \ldots \ldots . . . . . . .83$ 
V. Results and Discussion............................ 88

A. The $\pi^{*} \pi^{*}$ State of 8 -Chloroquinoline..................... 88

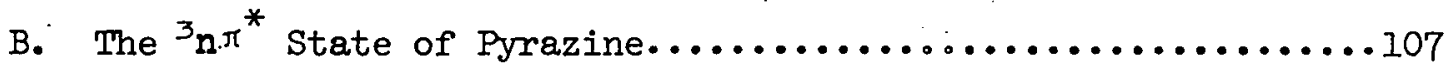

C. The ${ }^{3} \pi \pi^{*}$ State of Paradichlorobenzene...................120

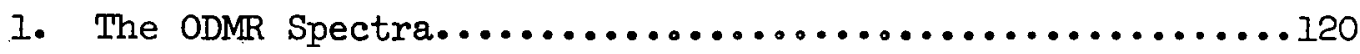

2. The PMDR spectra.................................

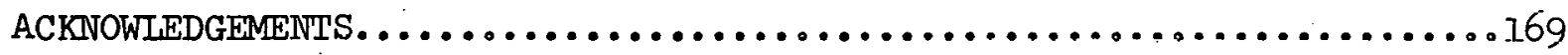

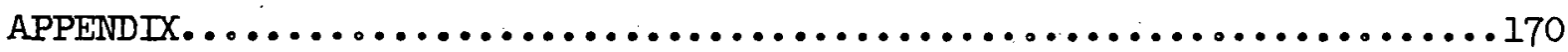

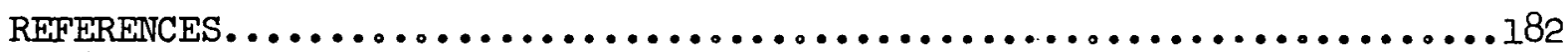


OPTICAILY DETECTED MAGNETIC RESONANCE OF MOLECULES

IN EXCITED TRIPLET STATES

Michael J. Buckley

Department of Chemistry, University of California, and Inorganic Materials Research Division, Lawrence Radiation Laboratory, Berkeley, California 94720

ABSTRACT

The sensitivity of optically detected magnetic resonance (ODMR) as a function of the intramolecular energy transfer processes is developed along with the explicit form of the spin Hamiltonian used in ODMR with zero external magnetic field. The main features of the optically detected ESR and ENDOR spectra in zero field for molecules with one $I=1$ or $I=3 / 2$ nuclear spin is presented.

The optically detected ESR spectra of the ${ }^{3} \pi \pi^{*}$ state of 8-chloroquinolint, the ${ }^{3} n \pi^{*}$ state of pyrazine and the ${ }^{3} \pi_{\pi^{*}}$ state of paradichlorobenzene are reported. In addition the ${ }^{35} \mathrm{Cl}$ and ${ }^{37} \mathrm{Cl}$ ENDOR spectra are reported for the excited triplet states of 8-chloroquinoline and paradichlorobenzene. The observed ODMR spectra are interpreted in terms of a spin Hamiltonian incorporating the electron spin-spin, nuclear quadrupole and nuclear-electron hyperfine interactions.

The ${ }^{3} \pi^{*}$ state of 8 -chloroquinoline is found to have essentially the same values for the electron spin-spin interaction as the ${ }^{3} \pi \pi^{*}$ state of 
quinoline. The chlorine nuclear quadrupole coupling constant of the ${ }^{3} \pi \pi^{*}$ state of 8-chloroquinoline is approximately the same as the values reported for the ground state of similar molecules, indicating that the chlorine electric field gradient is not significantly changed upon excitation.

The large electron spin-spin interaction measured for the ${ }^{3} n{ }^{*}$ state of pyrazine is explained in terms of the one-center contribution to the interaction. Analysis of the nitrogen hyperfine interaction gives the approximate values for the spin density of 0.28 and 0.35 for the nitrogen $\pi^{*}$ and $n$ orbitals respectively.

The ODMR and phosphorescence microwave double resonance (PMDR) spectra of paradichlorobenzene are consistent with the hypothesis that the excited state is a ${ }^{3} \pi \pi^{*}$ state and that the symmetry of the excited state is ${ }_{2 u}{ }^{\circ}$ Frum lise assigred urlentation of the electron spin-spin tensor, the spin density appears to be localized primarily on the four carbon atoms that are not bonded to the chlorines. The smald, value of the chlorine out-of-plane hyperfine element leads to the conclusion that the chlorines do not participate significantly in the excitation. On the basis of the PMDR spectra and the reduced value of the chlorine nuclear quadrupole coupling constant as compared to the value for the ground state, it is further hypothesized that the $\mathrm{C}-\mathrm{Cl}$ bonds are bent and that the molecule possesses $\mathrm{C}_{2 h}$ rather than. Dah spacial symmetry in the excited state. 


\section{INTRODUCTION}

Since the success of optically detected magnetic resonance (ODMR) of the lowest triplet state of organic molecules is highly dependent on the nature of the triplet state, a short review of some of the most important properties of the triplet state is given in this section. There are several very good review articles on the triplet state to which the reader is referred for a more complete discussion. ${ }^{1-3}$ The second half of this section is devoted to the historical development of ODMR and a survey of previous experimental results.

\section{A. The Excited Triplet State in Organic Molecules}

The ground state of most organic molecules consists of a singlet electron configuration in which all the electrons have their spins paired. The molecule may be excited to a higher energy electron configuration by the application of electromagnetic radiation of the appropriate energy. We will primarily be concerned with the excited electron configurations produced when one electron in the highest bonding molecular orbital $\left(\phi_{A}\right)$ is promoted to the lowest non-bonding molecular orbital $\left(\phi_{B}\right)$. Since electrons have a spin of $\frac{1}{2}$, there are four possible orientations for the two unpaired electrons, which, if we let $\alpha$ equal spin up and $\beta$ equal spin down, may be represented as,

$$
\begin{array}{lll}
\alpha(1) \alpha(2) & S_{z}=1 & S^{2}=1 \\
\alpha(1) \beta(2) & S_{z}=0 & S^{2}=0 \\
\beta(1) \alpha(2) & S_{z}=0 & S^{2}=0 \\
\beta(1) \quad \beta(2) & S_{z}=-1 & S^{2}=1
\end{array}
$$


This representation, however, is not satisfactory since the electrons obey Fermi-Dirac statistics and therefore the total wave function (orbital times spin) must be antisymetric with respect to electron exchange. In addition, we would like the spin functions to be eigenstates of $\mathrm{s}^{2}$ and $\mathrm{Sz}$. The spin functions $\alpha(1) \alpha(2)$ and $\beta(1) \beta(2)$ are clearly eigenstates of $\mathrm{S}^{2}$ and $\mathrm{Sz}$ since $\mathrm{S}^{2}=1$ for both and $\mathrm{Sz}=+1$ and -1 respectively. We can generate the $\mathrm{Sz}=0$ component of the triplet spin state by applying the lowering operator to the $\alpha(1) \alpha(2)$ state which gives us the desired spin function,

$$
{ }^{3} \psi_{0}=[1 / \sqrt{2}][\alpha(1) \beta(2)+\beta(1) \alpha(2)]
$$

The remaining spin function is a singlet

$$
I_{\psi}=[1 / \sqrt{2}][\alpha(1) \cdot \beta(2)-\beta(1) \alpha(2)]
$$

and, in cuntrast to the triplet spin funstions, is antioymmethrio will respect to electron exchange.

The spacial part of the excitcd state eleclron wävetunction may be respeserited as a symetric $(+)$ and antisymetric $(-)$ linear combination of $\phi_{A}$ and $\phi_{B}$ as:

$$
\psi_{ \pm}=[1 / \sqrt{2}]\left[\phi_{A}(1) \phi_{B}(2) \pm \phi_{A}(2) \phi_{B}(1)\right]
$$

Since the total wavefunction must be antisymetric, there are only four allowed representations of the total wavefunction; a singlet state with a symetric spacial function and an antisymetric spin function,

$$
{ }^{1} \bar{\Psi}=\left([1 / \sqrt{2}]\left[\phi_{A}(1) \phi_{B}(2)+\phi_{A}(2) \phi_{B}(1)\right]\right)([1 / \sqrt{2}][\alpha(1) \alpha(2)-\beta(1) \beta(2)])
$$


and a triplet state with an antisymetric spacial function and a symmetric spin function

$$
{ }^{\bar{\Psi}}=[1 / \sqrt{2}]\left[\phi_{A}(1) \phi_{B}(2)-\phi_{A}(2) \phi_{B}(1)\right] \cdot\left\{\begin{array}{l}
\alpha(1) \alpha(2) \\
{[1 / \sqrt{2}][\alpha(1) \beta(2)+\beta(1) \alpha(2)]} \\
\beta(1) \beta(2)
\end{array}\right\}
$$

The repulsive electrostatic-interaction between the two unpaired electrons gives rise to a term in the total Hamiltonian equal to $\mathrm{e}^{2} / \mathrm{r}_{12}$, where $e$ is the electron charge and $r_{12}$ is the vector connecting the two electrons. This term removes the degeneracy of the singlet and triplet states and results in the singlet state going to higher energy while the triplet state is shifted to lower energy with an energy separation between the two states of

$$
{ }^{1} E-{ }^{3} E=2 \delta_{12}
$$

where $\delta_{12}$ is the exchange integral given by

$$
\delta_{12}=\left\langle\phi_{A}(1) \phi_{B}(2)\left|\cdot e^{2} / r_{12}\right| \phi_{A}(2) \phi_{B}(1)\right\rangle
$$

For most organic molecules $2 \delta_{12}$ is 5000 to $10000 \mathrm{~cm}^{-. t}$. As will be discussed in the section on the spin Hamiltonian, the inclusion of the magnetic dipole-dipole interaction in the Hamiltonian removes the three fold degeneracy of the triplet state. This splitting is usually referred to as the zero field splitting and is on the order of $0.1 \mathrm{~cm}^{-1}$.

An additional contribution to the zero field splitting arises from the coupling of the spin and orbital electron angular momentum and is of the form $A(I \cdot s)$ where $I$ and $s$ are the spin and orbital angular momentum quantum numbers and $\mathrm{A}$ is a constant that depends on 
the particular molecule being considered. The effect of the spin orbit Hamiltonian is to mix states of different multiplicity and, therefore, to give singlet character to triplet states and vice versa. The most important effect of this is to permit the triplet state to undergo weak electric dipole radiation to the ground state (phosphorescence), the intensity from each of the three triplet sublevels being a function of the spin orbit coupling to both the excited and the ground singlet states.

Since the sensitivity of ODMR depends upon the number of molecules in their triplet state, an important consideration is intramolecular energy transfer processes. Following excitation, a molecule may lose energy by radiative or non-radiative pathways as shown in Figure 1. Phosphorescence $\left(T_{1} \rightarrow S_{0}\right)$ and fluorescence $\left(S_{1} \rightarrow S_{0}\right)$ comprise the radiative pathways and proceed with rate constants on the order if $10^{4}$ to $10^{-2} 000^{-1}$ and $10^{6}$ to $10^{9} \mathrm{sec}^{-1}$, respectively. The longer lifetime for phosphorescence resulto from the fact that. the triplet state is spin-forbidden for electric dipole radiation to the ground state.

The molecule may also lose energy through three non-radiative pathways.

1). Vibrational Relaxation -- or passage from a non-equilibrium vibrational. energy distribution in a given electronic state to the Boltzmann energy distribution relative to the zero point energy of that same state. This proceeds primarily by a non-radiative mechanism with a rate constant of approximately $1012 \mathrm{sec}^{-1}$

2) Internal Conversion -- or radiationless passage between two electronic states of the same spin multiplicity. The pathway also has a fast rate constant of approximately $10^{12} \mathrm{sec} .^{-1}$ 


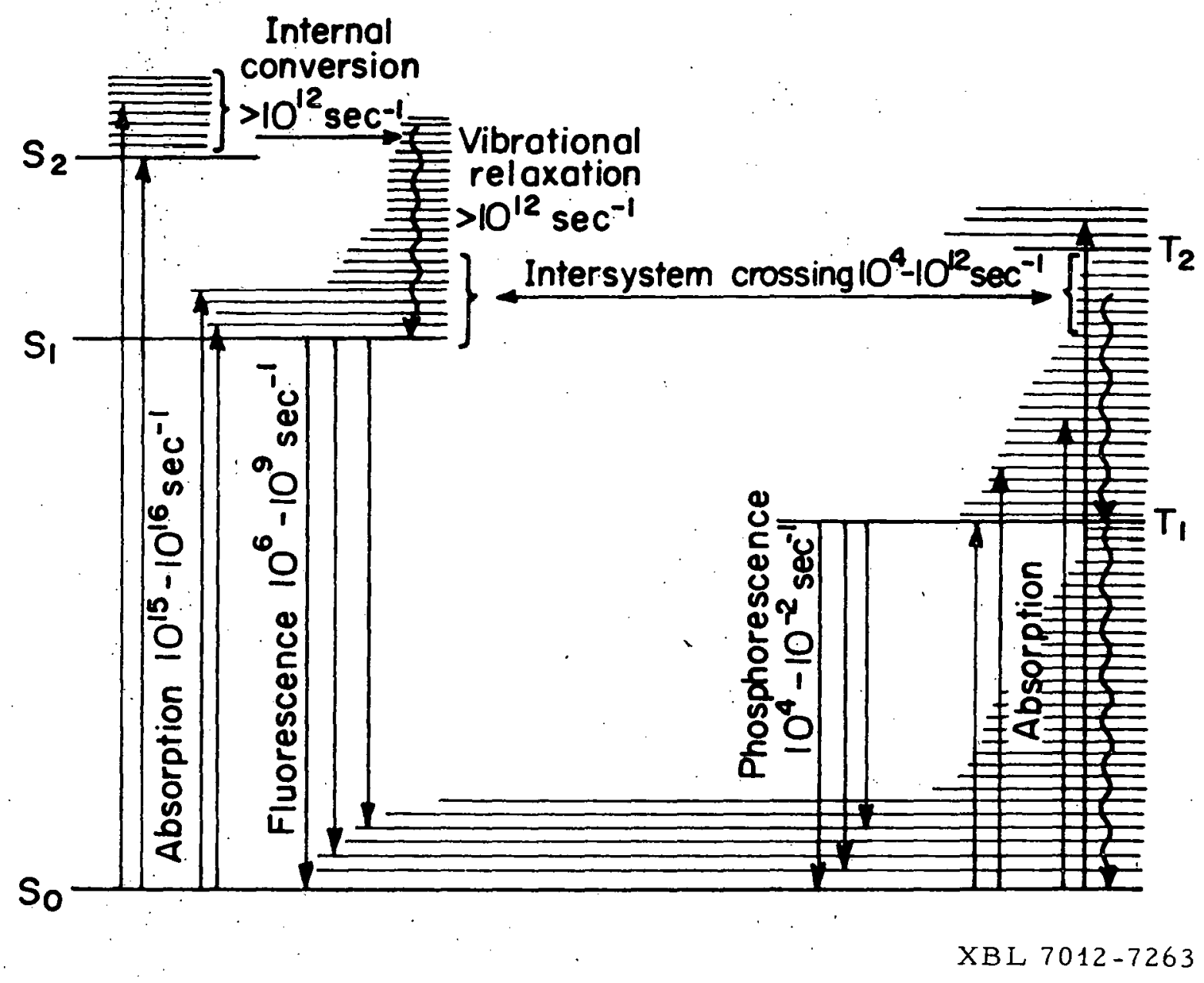

Figure 1

Intramolecular Energy Transfer Pathways and Rate Constants 
3) Intersystem Crossing -- or radiationless passage from an electronic state in the singlet manifold to an electronic state in the triplet manifold or vice versa. This pathway is slower than the other two and is on the order of $10^{4}$ to $10^{12}$ sec..$^{-1}$

The loweat triplet state may be populated either hy direct excitation into the triplet manifold followed by vibrational relaxation to the lowest vibrational level of $\mathrm{T}_{1}$ or by excitation into the singlet manifold followed by intersystem crossing into the triplet manifold. The latter is usually more efficient due to its larger cross section.

Although the exact mechanisms of intersystem crossing are not completely understood, it is generally found that at liquid helium temperatures $\left(4.20^{\circ} \mathrm{K}\right)$ the triplet sublevels of the lowest triplet state have unequal populations and consequently, a state of alignment exists for the electron spins. 4

'l'he various rate constants for energy lransfer, llue existence of spin allgnment, and the spin lattice relaxation rate between the triplet spin sublevels are all important factors in determining the sensitivity of ODMR as will be shown in section II.

\section{B. The Historical Development of ODMR}

The development of any field of science is difficult to trace since every advancement is dependent on the work of many previous researchers; however, we will choose for the starting point of this discussion the extensive study of the phosphorescence of organic molecules by Lewis and $\mathrm{Kasha}^{5,6}$ in 1944. In their series of papers it was proposed that the 
phosphorescent state of these molecules corresponded to their lowest triplet state. This hypothesis was strongly supported shortly thereafter by magnetic susceptibility measurements ${ }^{7,8}$ which showed that small changes in the susceptibility were observed upon irradiation of the samples.

As with any major change in the existing paradigm of science, this hypothesis was not universally accepted. 9 The most distressing aspect of the hypothesis was the failure to observe the predicted electron spin resonance (ESR) of the phosphorescent state. The problem was resolved in 1958 when Hut:hison and Mangum 10,11. succeeded in observing the ESR of naphthalene in its phosphorescent state and showed conclusively that the phosphorescent state was a triplet state. The experiment was performed by using conventional techniques in that the absorption of the microwave energy was monitored while varying the applied magnetic field. The sample, consisting of a single crystal of durene containing 2 to 5 mole percent naphthalene, was maintained at liquid nitrogen temperature while irradiating with a mercury arc lamp. The resonance signal was observed to decay with the same lifetime as the phosphorescence upon extinguishing the exciting light, proving that the phosphorescent state was being detected. The observed resonance spectra showed that the triplet state was already split into three levels in the absence of a magnetic field which explained the failure of previous experiments using randomly oriented samples to detect the resonance.

Subsequently, the triplet state ESR of many organic compounds was observed; however, most of the work was done on randomly oriented samples. Since only one parameter can usually be measured with randomly oriented 
samples, the separation of the three levels of the triplet could not be determined. In certain cases ${ }^{12,13}$ the three levels can be assigned but the assignment is difficult and the method has not been used often.

The limited sensitivity of ESR and the difficulty of preparing single crystal samples has restricted the number of molecules investigated. Only 14 molecules have been reported to date using conventional methods and they are all characterized by relatively long lived $\pi_{-\pi^{*}}$ triplet states (see Table I).

The next major change in the existing paradigm occurred in 1965 when Geschwind, Devlin, Cohen and Chinn ${ }^{14}$ reported the optical detection of the ESR of the excited metastable $\bar{E}(2 \mathrm{E})$ state of $\mathrm{Cr}^{+3}$ in $\mathrm{Al}_{2} \mathrm{O}_{3}$. In this classic experiment they showed that the optical rf double resonance techniques first suggested by Brossel and Kastler ${ }^{15}$ and widely used in gases $^{16}$ could also be applied to solids. The experiment was performed using a high resolution optical opectrometer to monitor the change in intensity of one of the Zeeman components of the fluorescent light $\left[\overline{\mathrm{E}}(2 \mathrm{E}) \rightarrow{ }^{4} \mathrm{~A}_{2}\right]$ as $\overline{\mathrm{E}}$ was saturated with microwaves when the magnetic field was swept through resonance. The resonance signal was observed by modulating the microwave field and detecting the resultant modulation of the optical emission, Since optical rather than microwave photons are detected, the sensitivity may be increased several orders of magnitude over conventional techniques. As an example, at temperatures below the $\lambda$ point of helium the resonance could be observed directly on an oscilloscope without the need for phase sensitive detection. The success in optically detecting the electron spin resonance of a metastable state 
led several research groups to attempt to apply the same principles to the optical detection of the ESR of organic molecules in their lowest triplet state.

In 1967 the first successful experiment was reported by Sharnoff for the $\Delta \mathrm{M}=2$ transition of naphthalene. ${ }^{17}$ In this experiment a single crystal of biphenyl containing 0.1 mole percent naphthalene was placed in a microwave cavity where it was inmersed in liquid helium maintained at $1.8^{\circ} \mathrm{K}$. The crystal was irradiated with the appropriately filtered light from a mercury arc lamp and the phosphorescence isolated with a detector consisting of a linear polarizer and a low resolution spectrometer. The microwave field was modulated at $40 \mathrm{~Hz}$ and the signal detected by feeding the output of the photomultiplier into a phase sensitive amplifier. In this experiment it was shown that the radiative matrix elements connecting any triplet sublevel with the ground singlet electronic level are functions of the magnetic quantum numbers of that sublevel.

At this point the development of ODMR of the lowest triplet state of organic molecules entered a new phase. Now that this new method was shown to be applicable to these molecules the research centered around improving the basic techniques and using this new tool to gain information on the various phenomena associated with the triplet state.

Shortly after Sharnoff's paper, Kwiram ${ }^{18}$ reported the optical detection of the $\Delta M=1$ and $\Delta M=2$ transitions of phenanthrene in its triplet state. In this investigation the experimental methods were the same as those used by Sharnoff except that the microwave field was not modulated while the exciting and emitted light was chopped antisynchronously at $50 \mathrm{~Hz}$. 
The $50 \mathrm{~Hz}$ output of the photomultiplier was converted to DC by a phase sensitive detector and fed into a signal averager. The observed change in intensity of the phosphorescence at the three transition frequencies was used to assign the spacial symetry of the triplet state.

Schmidt, Hesselmann, De Groot and van der Waals also reported the optical detection of quinoxaline $\left(d_{6}\right)$ in 1967. Their experimental procedure was basically the same as that used by Sharnoff, except that they modulated the magnetic field with and without amplitude modulation of the microwave field. They were able to show (1) that the emission originates from the top spin component (out-of-plane), and (2) from phosphorescence decay studies, that entry into the triplet state by intersystem crossing is also to the top spin component.

In 1968 Schmidt and van der Waals ${ }^{20}$ extended the almost zero fleld work (3G) of Hutchison's group ${ }^{2 l}$ by optically detecting the zero-field transitions of molecules in their triplet state at zero external magnetic field. Since it is necessary to vary the microwave frequency in order to observe the resonance in zero external magnetic field, a helix was used to couple the microwave power to the sample. The observed signals were extremely sharp and in the case of quinoxaline $\left(d_{6}\right)$, showed fine structure which was tentatively explained on the basis of a first order nitrogen nuclear quadrupole and second order nitrogen hyperfine interactions. The structure was explained quantitatively in a later paper ${ }^{22}$ in terms of a Hamiltonian incorporating these interactions.

Tint1, El-Sayed, Maki and Harris ${ }^{23}$ extended the method of optical detection in zero field by incorporating a high resolution spectrometer 
and studying the effect of the microwave field on the individual lines of the phosphorescence spectrum of 2,3-dichloroquinoxaline. They showed that the use of a high resolution spectrometer will give better sensitivity in cases where there is mixed polarization of the phosphorescence, since if the total emission is monitored, the change in intensity due to the microwave field may be partially cancelled. The sensitivity was excellent, and in fact, a very strong signal was observed using $C$. W. conditions for both the microwave and optical radiations. The observed structure of the zero-field transitions was explained quantitatively in a later paper 24 in which the first optically detected electron nuclear double resonance (ENDOR) for nitrogen was also reported. Several other papers followed on the observation and interpretation of nitrogen ENDOR in zero field 25,26 and was extended to ${ }^{35} \mathrm{Cl}$ and ${ }^{37} \mathrm{Cl}$ by Buckley and Harris ${ }^{27}$. Optical detection of electron-electron double resonance (EEDOR) was reported by Kuan, Tinti and El-Sayed 28 and was demonstrated to be a method of improving the signal strength of weak zero-field transitions if emission is from only one of the triplet sublevels.

Several interesting physical phenomena have been reported recently, including such areas as level anticrossing, 51 transferred hyperfine and nuclear quadrupole interactions from host to guest molecules ${ }^{52}$ and the prediction of microwave modulation of the phosphorescence. 53

As a consequence of the newness of this field most of the ODMR studies to date have been on molecules previously reported using conventional techniques (see Table 2). However, molecules with short triplet lifetimes which cunnot be observed by conventional methods have received considerable 
attention and the resonances of several new molecules have been reported (see Tabie 3).

One of the most promising new applications of magnetic resonance is the investigation of exciton interactions in crystals. Wolf and his coworkers using conventional ESR techniques have observed energy exchange between pairs of naphthalene ( $h_{8}$ ) molecules as nearest neighbors in an 1sotoplcally dilute system, ${ }^{29}$ and triplet excitons in pure crystals of naphthalene and anthracene single crystals. ${ }^{\text {th }}$ sharnor has reported the ODMR of triplet exitons in a single crystal of benzophenone ${ }^{34-35}$; however, his results have been questioned ${ }^{51}$ and to date no other reports have been published of the ODMR of excitons in molecular crystals.*

In conclusion, ODMR has developed into three basic areas: 1) the study of the electron distribullun of. oiganic molecules in their triplet. state by analysis of the zero field, nuclear quadrupole and hyperfine interactions, 2) investigations into the intramolecular as well as intermolecular pathways and rates of energy transfer in trap molecules by analysis of the ODMR signal as a function of time for various vibronic bands in the phosphorescence spectrum, and 3) as a tool to investigate the energy levels and dynamic properties of exciton bands in molecular crystals.

* Recently, however, Francis and Harris ${ }^{76}$ have used this technique to measure the density of states functions of triplet Frenkel excitons in molecular crystals and have observed coherent migration. 
Table 1

Single Crystal ESR Studies

Molecule

Anthracene

Benzene

Diphenylmethylene

Diphenylmethylene

Fluorene

Fluorenylidene

Isoquinoline

Mesitylene

Naphthalene-hs

Naphthalene- $h_{8}$

Naphthalene-da

Phenanthrene

Phenanthrene

Phenazine

Fyrene-hio

Pyrene-dio

Quinoxaline

Quinoline

Tetramethylpyrazine
Host Crystal

$$
\mathrm{T}\left({ }^{\circ} \mathrm{K}\right) \quad \mathrm{D}(\mathrm{GHz})^{*} \mathrm{E}(\mathrm{GHz})^{*} \quad \operatorname{Ref} .
$$

Diphenyl

$$
77+2.1453-.253 \quad 55
$$

Benzene- $d_{6}$.

$1.95+4.740-.192$

93

Benzophenone

$77 \pm 12.1430 \mp .5750 \quad 46-48$

1,1-Diphenylethylene

77

Fluroene

Diazafluorene

Durene

B-Trimethylborazole

Durene

Biphenyl

$\pm 2.9140 \mp 1.3341$

$46-48$

$77 \pm 12.2683 \mp .8478 \quad 47,48$

$77 \pm 3.0099 \mp .3508 \quad 43$

$77 \pm 1.8586 \mp .7195 \quad 78$

$77+3.0069-.410710,11,40,41$

$77+2.9739-.463210,11,40,41$

$+2.9799-.461710,11,40,41$

Durene- $d_{14}$

$77+3.03807-.417610,11,40,41$

$+3.0279-.401710,11,40,41$

Biphenyl

$78 \pm 3.01079 \mp 1.396321$

$\pm 3.0111 \mp 1.396110,11,40,41$

$\pm 3.0219 \mp 1.400010,11,40,41$

Fluorene

$\pm 3.0129$

71.4000

$10,11,40,41$

Diphenyl

$90+2.2304-.329849$

F'luorene

$100 \pm 2.0326 \mp .9479 \quad 44$

Fluorene

room $\pm 1.9717 \mp .9479 \quad 45$

temp

Durene

$77 \pm 3.0189 \mp .5456 \quad 42$

Durene

$77 \pm 3.0878 \mp .4857 \quad 43$

$77 \pm 2.9679 \quad \mp .1289 \quad 50$

* The zero field splitting parameters $D$ and $E$ are discussed in Section III, pp. 33-40. 
Table 2

ODMR of Molecules Previously Observed by Conventional Techniques

\begin{tabular}{lc}
\multicolumn{1}{c}{ Molecule } & Reference \\
Naphthalene & 17 \\
Quinoxaline & 19 \\
Quinoline & 39 \\
Phenanthrene & 18 \\
Isoquinoline & 39 \\
Tetramethylpyrazine & 20
\end{tabular}

Table 3

New Molemules Observed using ODMR

Molecule

Benzophenone ${ }^{a}$

2,3 dichloroquinoxaline

8-chloroquinoline

Pyrazine $-\mathrm{h}_{4}$

Pyrazine $-d_{4}$

a Pure crystal

b

For the second trap
Host

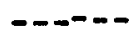

Durene

DDurene

Paradichlorobenzene Paradichlorobenzene
$\mathrm{T}\left({ }^{\circ} \mathrm{K}\right) \quad \mathrm{D}(\mathrm{GHz}) \quad \mathrm{E}(\mathrm{GHz}) \quad$ Ref.

$4.2+4.557-.630 \quad 34$

$+4.4309-.4977 \quad 35$

$1.6+2.9849-.527124$

$1.7+2.9550-.4295 b 26$

$1.6+2.9526^{b}-.4248^{b} 36$

$1.3+9.264-.180 \quad 37$

$4.2+10.170-.216 \quad 38$

$4.2+10.204-.206 \quad 38$ 
II. SENSITIVITY CONSIDERATIONS IN OPTICAL DETECTION

In this section the basic proceduresused in the optical detection of ESR and ENDOR in zero magnetic field are reviewed. Quantitative expressions are derived for the change in phosphorescence intensity as a function of the various relaxation rate constants for the triplet state and the strength of the applied microwave field.

\section{A. ESR}

The experiments were all performed under conditions of continuous optical excitation while monitoring the change in intensity of the phosphorescence as a function of the applied microwave fieid. Only the case in which the triplet state is populated by excitation of the sample into the first excited singlet state followed by intersystem crossing into the triplet state will be considered. For molecules with reasonably high symmetry (i.e., $D_{2 h}, C_{2 h}$, and $C_{2 v}$ ) different methods of populating the triplet state may produce different spin alignments; however, the same considerations apply in calculating the sensitivity achieved using ODMR. $63,66,67$

The radiative and non-radiative pathways for energy transfer are depicted in Figure 2, where $S_{1}$ is the population of the lowest excited singlet state, $N_{\chi}(x=x, y, z)$ is the steady state population of the corresponding triplet levels, $\mathrm{K}_{\mathbf{I} X}$ is the non-radiative relaxation rate constant for relaxation to $S_{0}, K_{\chi}$ is the radiative or phosphorescence rate constant for relaxation to $S_{0}, W_{x_{1} x_{2}}\left(x_{1} \neq x_{2}\right)$ is the spin lattice relaxation rate constant and $P_{x_{2} x_{2}}\left(x_{1} \neq \neq_{2}\right)$ is the induced rate constant due 


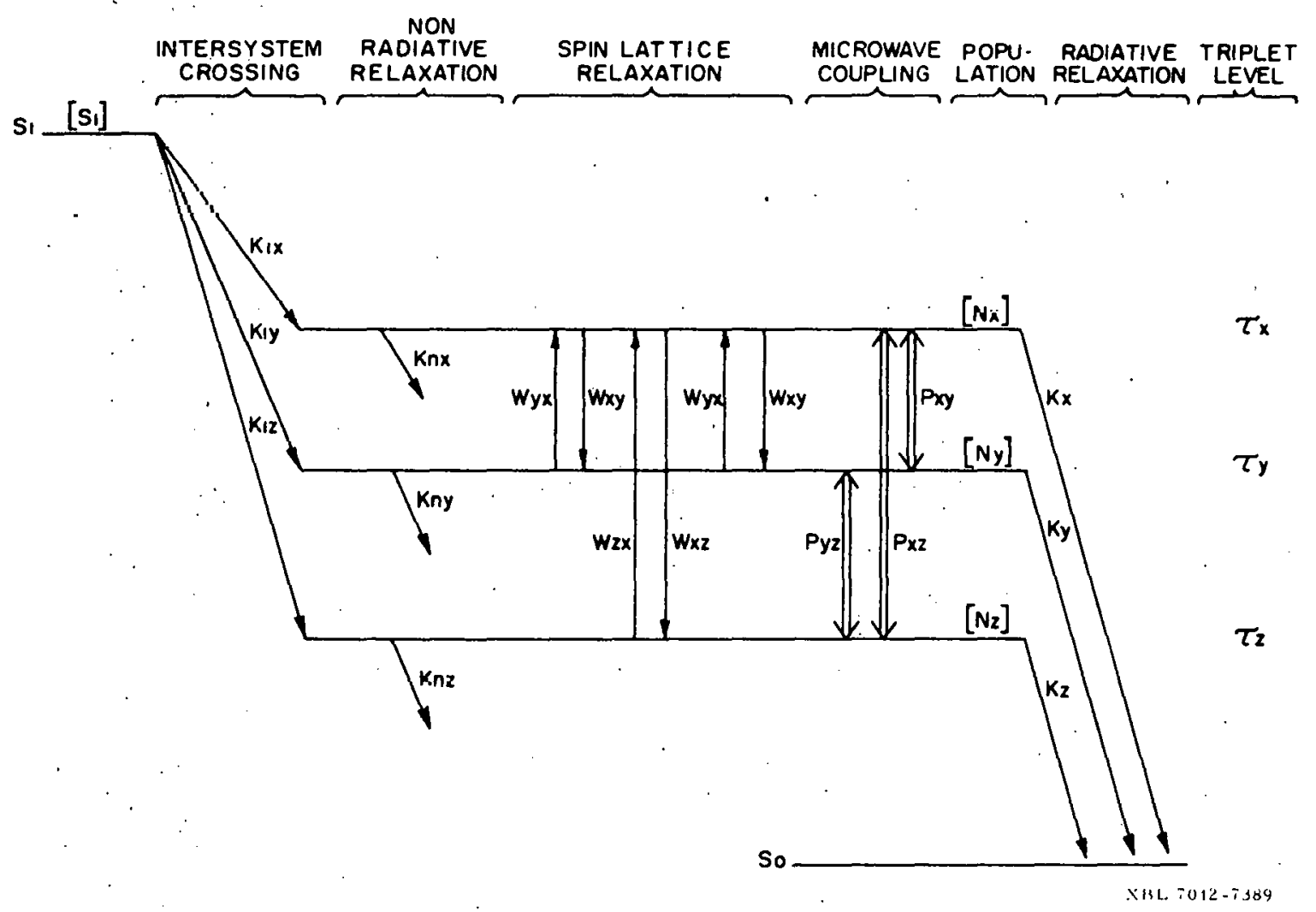

Figure 2

Relaxation Pathways and Rate Constants for the Triplet State 
to the applied microwave field $\left(\mathrm{H}_{2}\right)$.

When the microwave field does not connect any two of the zero field levels of the triplet, the steady state population is given by setting $P_{\chi_{1} \chi_{2}}=0$. The application of the microwave field at a frequency corresponding to the energy separation of two of the levels (i.e., $\nu=E_{x}-E_{y} / h$ ) will introduce a new pathway for relaxation causing redistribution of the population which in most cases results in a change in the phosphorescence intensity.

Since optical, rather than microwave, photons are detected, one would expect the sensitivity to be improved in proportion to the ratio of the energies of the photons, which, for a typical molecule, is approximately $3 \times 10^{5}$. The detual change in the phosphorescence intensity, however, is a complex function of the various relaxation rate constants. Therefore, the actual improvement in sensitivity (if there is an improvement) will depend on the molecule under study.

In order to derive a reasonably simple quantitative expression for the change in intensity of the phosphorescence, the three following assumptions will be made:

1) The splitting of the three triplet zero field levels by nuclear quadrupole and nuclear hyperfine interactions will be neglected,

2) Only the two levels connected by the $\mathrm{H}_{2}$ field ( $\tau_{x}$ and $\left.\tau_{y}\right)$ will be considered, and

3) Only the steady state condition $d N_{x} / d t=d N_{y} / d t=0$ will be considered for both the case when $\mathrm{H}_{2}=0$ and $\mathrm{H}_{1} \neq 0$.

The first assumption will predict too great a change in intensity if the individual triplet levels are split by more than the frequency 
width of the $\mathrm{H}_{1}$ field, since in this case the $\mathrm{H}_{2}$ field will induce an additional relaxation pathway for only a fraction of the population of each. triplet level at any given frequency.

The second assumption will introduce an error in the expression fur the percentage change in intensity since the intensity contribution from the level not connected by the $\mathrm{H}_{1}$ field $\left(\tau_{z}\right)$ is neglected. I'his assumption also requires that the spin lattice relaxation rate between $\tau_{z}$ and ${ }^{\top} \mathrm{x}$ and belween $\tau_{\mathrm{L}}$ and ${ }_{{ }^{\prime}}$ be neglected. This is uoually valid since the experiments are performed at or below $4.20^{\circ} \mathrm{K}$.

The third assumption requires that the experiment be performcd uaing C. W. microwave conditions or modulating the microwave field with a frequency lower than the total rate constant of the system.

The differential equations describing the population of the levels shown in Figure 3 are

$$
\begin{aligned}
& \frac{d N_{x}}{d t}=S_{1} K_{1 x}-N_{x}\left[K_{n x}+K_{x}+W_{x y}+P_{x y}\right]+N_{y}\left[W_{y x}+P_{x y}\right] \\
& \frac{d N_{y}}{d t}=S_{1} K_{1 y}-N_{y}\left[K_{1 l y}+K_{y}+W_{y x}+P_{x y}\right]+N_{x}\left[W_{x y}+P_{x y}\right] \ldots
\end{aligned}
$$

With the definitions

$$
\begin{aligned}
& \Lambda=K_{n x}+K_{x}+W_{x y}+P_{x y} \\
& B=W_{y x}+P_{x y} \\
& C=K_{n y}+K_{y}+W_{y x}+P_{x y} \\
& D=W_{x y}+P_{x y}
\end{aligned}
$$

Equations 1 and 2 may be rewritten 


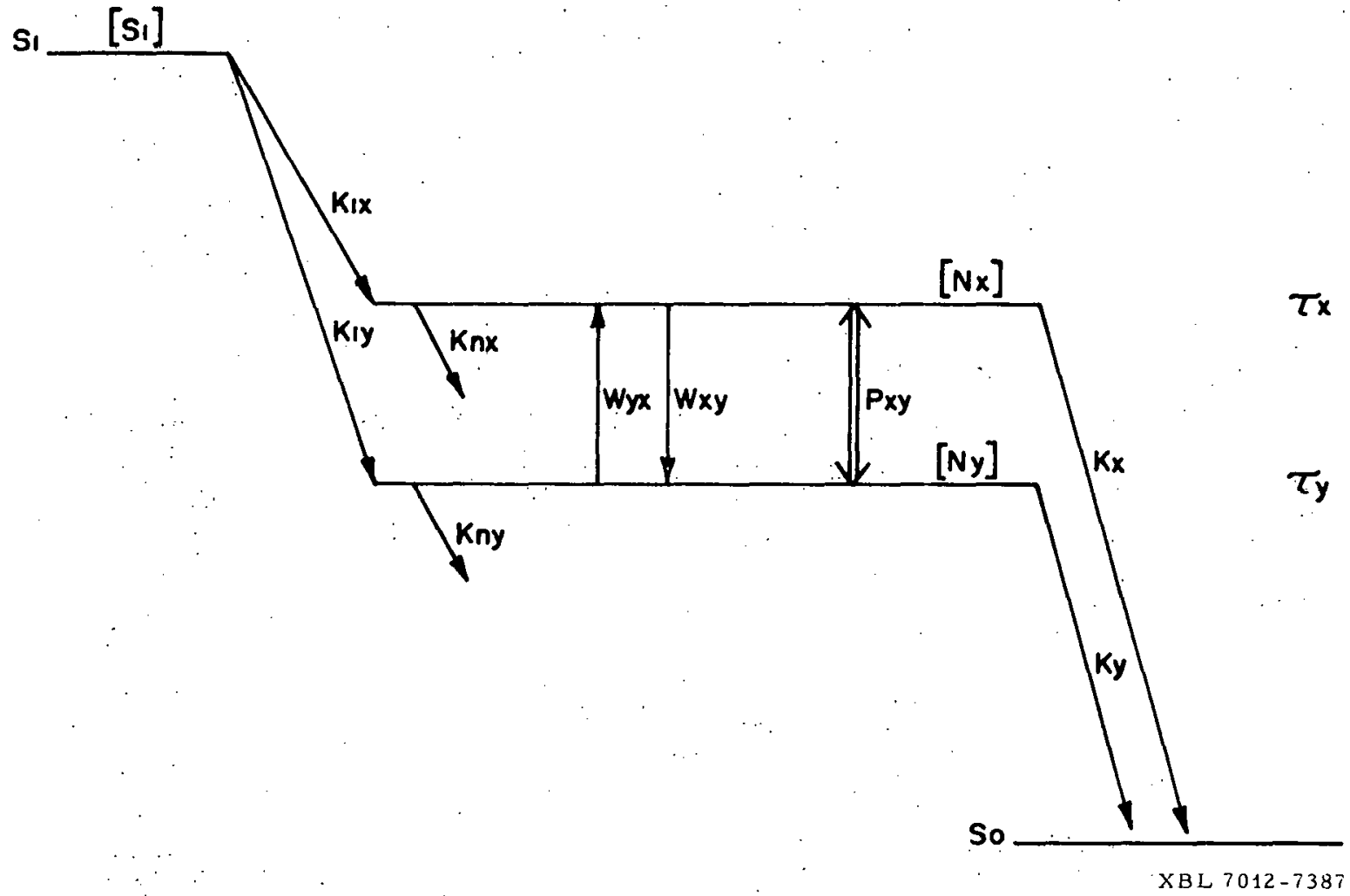

Figure 3

Relaxation Pathways and Rate Constants for only 'Two of the Three Triplet Levels (See Text) 


$$
\begin{aligned}
& \frac{d N}{d t}=S_{1} K_{1 x}-N_{x} A+N_{y} B \\
& \frac{d N}{d t}=S_{1} K_{1 y}-N_{y} C+N_{x} D
\end{aligned}
$$

The steady state assumption allows us to write

$$
\begin{aligned}
& \frac{d N_{x}}{d t}=S_{I} K_{I x}-N_{x} A+N_{y} B=0 \\
& \frac{d N_{y}}{d t}=S_{I} K_{I y}-N_{y} C+N_{x} D=0
\end{aligned}
$$

Upon solving Equations (6) and (7) for the population of the triplet levels, we have

$$
N_{x}=\frac{S_{1}\left[C_{1 x}+B K_{1 y}\right]}{A C-B D}
$$

and

$$
N_{y}=\frac{S_{1}\left[A K_{1 y}+D K_{1 x}\right]}{A C-B D}
$$

The intensity of the phosphorescence detected with an optical spectrometer may be written

$$
I:-a_{1} N_{x} K_{x}+a_{2} N_{y} K_{y}
$$

where $a_{1}$ and $a_{2}$ are constants that depend on the polarization of the emission, the orientation of the sample, and the efficiency of the detection system. The assumption will be made that $a_{1}=a_{2}$, which allows the fractional change in the intensity of the phosphorescence upon application of the $\mathrm{H}_{1}$ field to be written 


$$
\Delta I=\frac{I-I_{0}}{I_{0}}=\frac{I}{I_{0}}-I
$$

where $I_{0}$ is the intensity of the phosphorescence when $P_{x y}=0$. With this condition, it is convenient to define the parameters given in Equation

3 as

$$
\begin{aligned}
& a=K_{n x}+K_{x}+w_{x y} \\
& b=w_{y x} \\
& c=K_{n y}+K_{y}+w_{y x} \\
& d=w_{x y}
\end{aligned}
$$

If both of the triplet levels are monitored, the fractional change in intensity of the emission is given by

$$
\Delta I=\frac{\left[K_{1 y}\left(A K_{y}+B K_{x}\right)+K_{1 x}\left(\mathrm{CK}_{x}+D K_{y}\right)\right][a c-b d]}{\left[K_{1 y}\left(a K_{y}+b K_{x}\right)+K_{1 x}\left(c K_{x}+d K_{y}\right)\right][A C-B D]}-1 \text {. }
$$

In some cases it is possible to monitor only one of the triplet levels connected by the $\mathrm{H}_{1}$ field, in which case the changes in intensity of emmission from the $\tau_{\mathrm{x}}$ and $\tau_{\mathrm{y}}$ levels are given by

$$
\Delta I_{x}=\frac{\left[C K_{1 x}+B K_{1 y}\right][a c-b d]}{\left[c K_{1 x}+b K_{1 y}\right][A C-B D]}-1
$$

and $\quad \Delta I_{y}=\frac{\left[A K_{1 y}+D K_{1 x}\right][a c-b d]}{\left[a K_{1 y}+d K_{1 x}\right][A C-B D]}-1$ 
Three limiting cases will now be discussed in order to examine the effect of the magnitude of the various rate constants on the sensitivity of the experiment.

Case \#l, The Effect of the Radiative Rate Constants

For this case the additional assumption is made that the nonradiative and spin lattice relaxation may be neglected. The parameters defined in Equations 3 and 12 become
$A=K_{x}+P_{x y}$
$\mathrm{a}=\mathrm{K}_{\mathrm{x}}$
$B=P_{x y}$
$\mathrm{b}=0$
$C=K_{y}+P_{x y}$
$c=\mathrm{K}_{\mathrm{y}}$
$D=P_{y x}$
$d=0$

In the absence of the $\mathrm{H}_{1}$ field the steady state populations are given by

$$
\begin{aligned}
& \mathrm{N}_{\mathbf{x}}^{0}=\mathrm{S}_{2}\left(\mathrm{~K}_{1 \mathrm{x}} / \mathrm{K}_{\mathrm{x}}\right) \\
& \mathrm{N}_{\mathrm{y}}^{0}=\mathrm{S}_{1}\left(\mathrm{~K}_{1 \mathrm{y}} / \mathrm{K}_{\mathrm{y}}\right)
\end{aligned}
$$

The steady state population of ${ }^{\tau} \mathrm{X}$ is given by Equation 8 which for this example becomes

$$
N_{x}=\frac{S_{1}\left[K_{1 x} K_{y}+P_{x y}\left(K_{1 x}+K_{1 y}\right]\right.}{\left[K_{x} K_{y}+P_{x y}\left(K_{x}+K_{y}\right)\right]}
$$

In the limit that $\mathrm{P}_{x y}$ is much larger than any of the relaxation rate constants, the populations of $\tau^{\top} \mathrm{x}$ and ${ }^{\top} \mathrm{y}$ are equalized and the 
transition is saturated. Clearly, the power required to equalize the populations is directly proportional to the relaxation rate of the system and inversely proportional to the lifetime of the excited state.

The population of ${ }^{\tau} \mathbf{x}$ at saturation is given by

$$
N_{x}^{s}=\frac{s_{1}\left[K_{1 x}+K_{2 y}\right]}{\left[K_{x}+K_{y}\right]}
$$

and the corresponding population of ${ }^{\prime}{ }^{y}$ is given by

$$
\mathrm{N}_{\mathrm{y}}^{\mathrm{s}}=\frac{\mathrm{S}_{1}\left[\mathrm{~K}_{1 \mathrm{x}}+\mathrm{K}_{1 \mathrm{y}}\right]}{\left[\mathrm{K}_{\mathrm{x}}+\mathrm{K}_{\mathrm{y}}\right]}
$$

and therefore, $\mathrm{N}_{\mathrm{x}}^{\mathrm{S}}=\mathrm{N}_{\mathrm{y}}^{\mathrm{S}}$.

The change in population of ${ }^{T}{ }_{x}$ upon saturation is given by

$$
\Delta N_{x}=N_{x}^{s}-N_{x}^{0}=\frac{K_{x} K_{y y}-K_{y} K_{l x}}{K_{l x}\left(K_{x}+K_{y}\right)} .
$$

Therefore, if $\mathrm{K}_{x} \mathrm{~K}_{\mathrm{ly}}=\mathrm{K}_{\mathrm{y}} \mathrm{K}_{2 x}$, there is no change in population.

If the emissions from $\tau_{\mathrm{x}}$ and $\tau_{\mathrm{y}}$ are monitored simultaneously, ' the fractional change in intensity is given by Equation 13 which, for this example, reduces to

$$
\begin{aligned}
\Delta I & =\frac{\left[K_{l y}\left(A K_{y}+B K_{x}\right)+K_{l x}\left(C K_{x}+D K_{y}\right)\right]}{\left[K_{l y}(A C-B D)\right]}-1 \\
& =\frac{\left[K_{l x}+K_{l y}\right]\left[P_{x y}\left(K_{x}+K_{y}\right)+K_{x} K_{y}\right]}{\left[K_{l x}+K_{l y}\right]\left[P_{x y}\left(K_{x}+K_{y}\right)+K_{x} K_{y}\right]}-1
\end{aligned}
$$


And therefore, $\Delta I=0$ and no change in the intensity of emission will be observed.

However, if a high resolution optical spectrometer is used, it is often possible to monitor the emission from just one of the triplet levels via its selective emission to the origin or a vibration of the ground state singlet manifold. Consider for example, ${ }^{\prime} \mathrm{x}$, in which case, the change in intensity given by Equation 14 becomes.

$$
\Delta I_{x}=\frac{K_{x}}{K_{l x}}\left[\frac{P_{x y}\left(K_{l x}+K_{l y}\right)+K_{x} K}{P_{x y}\left(K_{x}+K_{y}\right)+K_{x} K_{y}}\right]-I \text {. }
$$

In the limiting case where intersystem crossing proceeds primarily to $T_{\mathrm{x}}\left(\mathrm{K}_{2 \mathrm{x}} \gg \mathrm{K}_{2 \mathrm{y}}\right)$ Equation 24 reduces to

$$
\Delta I_{x}=\frac{P_{x y x} K_{x}+K_{x} K}{P_{x y}\left(K_{x}+K_{y}\right)+K_{x} K_{y}}-1
$$

At saturation we have

$$
\Delta I_{x}^{s}=\frac{K_{x}}{K_{x}+K_{y}}-I
$$

The effect of the ratio of the radiative rate constanto $\left(\mathrm{K}_{\mathrm{x}} / \mathrm{K}_{\mathrm{y}}\right)$ on the maximum change in intensity of the emission may be illustrated with the following examples:

$\begin{array}{cr}\mathrm{K}_{\mathrm{x}} / \mathrm{K}_{\mathrm{y}} & \Delta I(\%) \\ 0.1 & 91 \\ 1 & 50 \\ 10 & 9\end{array}$


Therefore, the maximum sensitivity is achieved if the level with the fast intersystem crossing rate constant has the slower phosphorescence rate constant. Unfortunately, the opposite is generally found to be the case.

Case \#2, The Effect of Spin Lattice Relaxation

The two rate constants for spin lattice relaxation are not independent and may be related directly to the spin lattice relaxation time $\mathrm{T}_{1}$ for any given temperature. 64,65

The interaction between the energy and the lattice may be represented schematicallỳ as

SPINS

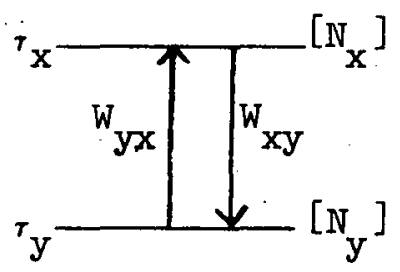

LATTICE

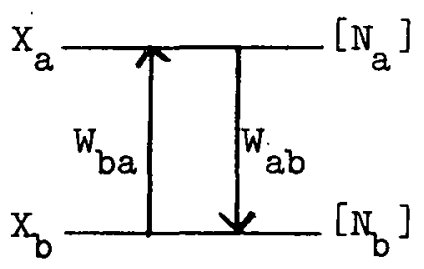

The conservation of energy requires that for each transition from ${ }^{\top} \mathbf{x}$ to $\tau_{\mathrm{y}}$ there be a corresponding lattice transition from $\mathrm{X}_{\mathrm{b}}$ to $\mathrm{x}_{\mathrm{a}}$ and vice versa. The transition rate for the lattice may be written

$$
\begin{aligned}
& \mathrm{w}_{\mathrm{ab}}=\mathrm{N}_{\mathrm{a}} \mathrm{A} \\
& \mathrm{w}_{\mathrm{ba}}=\mathrm{N}_{\mathrm{b}} \mathrm{A}
\end{aligned}
$$

where $A$ is the transition probability and $N_{a}$ and $N_{b}$ are the populations of $\mathrm{X}_{\mathrm{a}}$ and $\mathrm{X}_{\mathrm{b}}$ respectively. 
The spin lattice relaxation rate constants may be written in terms of the population of the lattice as

$$
\begin{aligned}
& \mathrm{w}_{x y}=\mathrm{w}_{b a}=\mathrm{N}_{b} \mathrm{~A} \\
& \mathrm{w}_{\mathrm{yz}}=\mathrm{w}_{\mathrm{ab}}=\mathrm{N}_{a} \mathrm{~A}
\end{aligned}
$$

Since the lattice is at the temperature of the bath (Iiquid helium), the nomallzed. pupulation of the lattice i. given by

$$
\begin{aligned}
& N_{a}=\frac{e^{-\delta / 2 k t}}{e^{-\delta / 2 k t}+e^{\delta / 2 k t}-f} \\
& N_{b}=\frac{e^{\delta / 2 k t}}{e^{-\delta / 2 k t}+e^{\delta / 2 k t}}=1-f
\end{aligned}
$$

where $\delta=(E x-E y) / 2$ and $E x$ and Ey are the energies of the $\tau_{\mathbf{x}}$, and $\tau \mathrm{y}$ levels respectively. The spin lattice relaxation rates may now be written

$$
\begin{aligned}
w_{x y} & =(1-f) A \\
w_{y x} & =(f) A
\end{aligned}
$$

The opin lattice relaxation time is defined by the expression

$$
\mathrm{T}_{1}=\frac{1}{\mathrm{~W}_{\mathrm{xy}}+\mathrm{W}_{\mathrm{yx}}}=\frac{i}{\mathrm{~A}} \text {. }
$$

Therefore, $W_{x y}$ and $W_{y x}$ may be expressed in terms of $T_{1}$ and $f$, as

$$
\begin{aligned}
& \mathrm{w}_{x y}=\frac{1-f}{T_{1}} \\
& w_{y x}=\frac{f}{T_{1}}
\end{aligned}
$$


In the derivation of equation 32 it is assumed that only a direct process of energy transfer between the spin system and the lattice exists which is usually the case at the temperatures of the experiments $\left(4.2^{\circ}\right.$ to $\left.1.3^{\circ} \mathrm{K}\right)$. In the case that Raman or Orbach processes are present, only the explicit temperature dependence of the relaxation must be corrected so that the spin lattice relaxation may always be defined for a two level system in terms of only $T_{1}$ at a given temperature.

A short $\mathrm{T}_{1}$ relaxation time will tend to produce a Boltzmann population distribution between the spin sublevels and will therefore generally reduce the spin alignment. This can be seen by considering the simple case where there is only intersystem crossing to $\tau_{\mathrm{y}}$ and emission from ${ }^{\top} \mathrm{x}$ and ${ }^{\top} \mathrm{y}$. Again the non-radiative relaxation rate constants $\mathrm{K}_{1 \mathrm{x}}$ and $\mathrm{K}_{\mathrm{ly}}$ are assumed to be negligible.

The parameters defining this model are

$$
\begin{array}{ll}
A=K_{x}+W_{x y}+P_{x y} & a=K_{x}+W_{x y} \\
B=W_{y x}+P_{x y} & b=W_{y x} \\
C=K_{y}+W_{y x}+P_{x y} & c=K_{y}+W_{y x} \\
D=W_{x y}+P_{x y} & d=W_{x y}
\end{array}
$$

and the populations of $r_{x}$ and $\tau_{y}$ when $P_{x y}=0$ are given by

and

$$
\begin{aligned}
N_{x}^{\circ} & =\frac{S_{1}\left[\left(K_{y}+W_{y x}\right) K_{l x}\right]}{K_{x y}+K_{y} W_{x y}+K_{x} W_{y x}} \\
N_{y}^{\circ} & =\frac{S_{1}\left[\left(W_{x y}\right) K_{1 x}\right]}{K_{x} K_{y}+K_{y} W_{x y}+K_{x} W_{y x}}
\end{aligned}
$$


In the limit that. $W_{x y}=W_{y x}=0$ this reduces to

$$
\begin{aligned}
& \mathrm{N}_{\mathrm{x}}^{0}=\frac{\mathrm{S}_{1}\left[\mathrm{~K}_{1 \mathrm{x}}\right]}{\mathrm{K}_{\mathrm{x}}} \\
& \mathrm{N}_{\mathrm{y}}^{0}=0
\end{aligned}
$$

At high temperatures when $\mathrm{w}_{\mathrm{xy}} \approx \mathrm{w}_{\mathrm{yx}} \gg \mathrm{K}_{\mathrm{x}}, \mathrm{K}_{\mathrm{y}}, \mathrm{K}_{\mathrm{lx}}$, Equation 34 becomes

$$
\begin{aligned}
& N_{x}^{n}=\frac{S_{1}\left[K_{1 x}\right]}{K_{x}+K_{y}} \\
& N_{y}^{O}=\frac{S_{1}\left[K_{1 x}\right]}{K_{x}+K_{y}}
\end{aligned}
$$

Since the change in population is monitored, it is clearly advantageous to perform the experiments at the lowest possible temperature in order to decrease the thermalization of the spin levels and the resulting loss in sensitivity.

Case \#3, The Effect of Non-Radiative Relaxation

The final case to be considered is the effect of the non-radiative relaxation rate constants $K_{l x}$ and $K_{l y}$ on the sensitivity of the experiment. It is obvious that since only the radiative emission is detected; a large rate of depopulation by non-radiative relaxation is not desirable. In the case of a sample that rclaxco primarily through non-radiative pathways, the sensitivity may be improved by using conventional ESR techniques and monitoring the absorption of microwave power, or in extreme cases by monitoring the change in 
temperature of the sample. A quantitative measure of the decrease in sensitivity may be calculated by substituting the appropriate rate constants into Equations 13, 14 and 15; however the expressions are rather complex and therefore not particularly useful.

\section{B. ENDOR}

The sensitivity of this experiment may be simply estimated if the assumption is made that there is no nuclear polarization. Since this assumption has yet to be thoroughly investigated, it is reasonable to expect that in some cases it will not be valid. Nuclear polarization may arise through cross relaxation between the electron and nuclear spin systems (the Overhauser effect), or it may be.induced by saturation of "forbidden" transitions (simultaneous electron nuclear flips). It is also possible that selective intersystem crossing may preferentially populate a particular nuclear spin level if there is strong hyperfine coupling of the electron and nuclear wavefunctions.

In the absence of nuclear polarization, the sensitivity of the optically detected ENDOR signal may be understood by referring to Figure 4 in which the ${ }^{\tau_{x}}$ and ${ }^{\tau_{y}}$ triplet levels are now each composed of two levels. This splitting of the triplet levels is due to nuclear quadrupole and hyperfine interactions as will be discussed in the section on the spin Hamiltonian.

The results obtained by considering the triplet levels as being split into only two nuclear sublevels are independent of the number of sublevels if the ESR transition connects only one nuclear sublevel in 


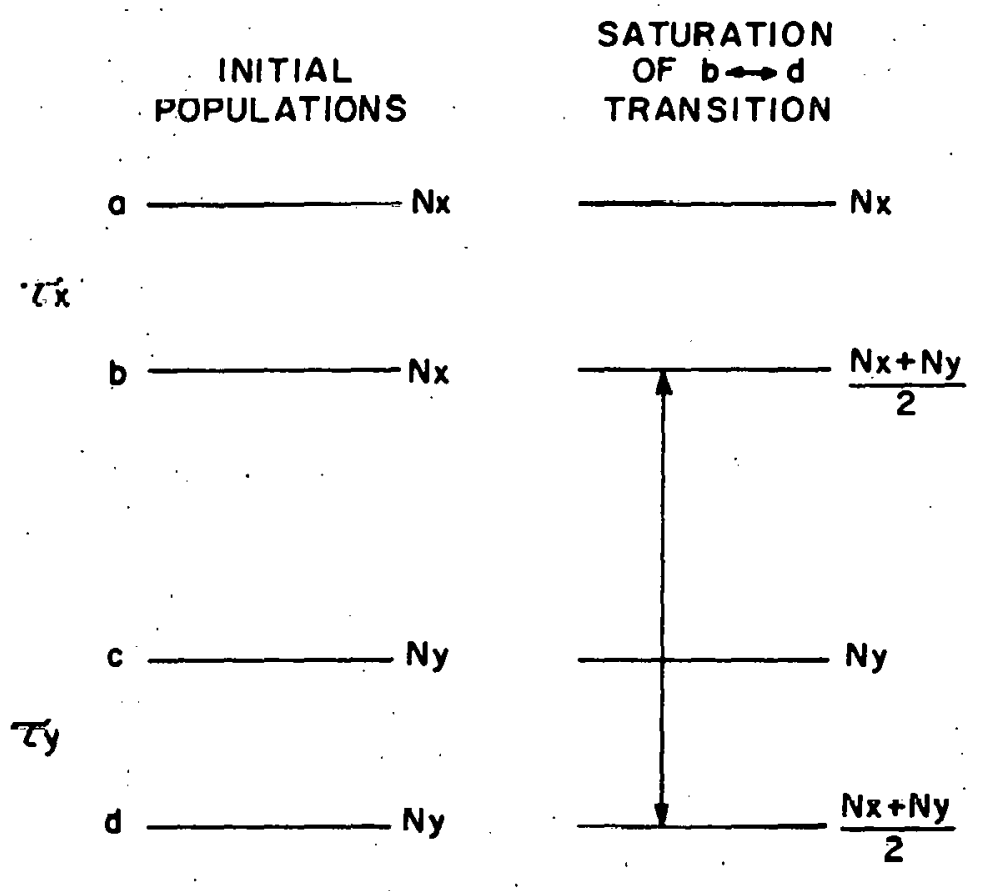

SATURATION OF $h \leftrightarrow d$ and $a \leftrightarrow b$

TRANSITIONS

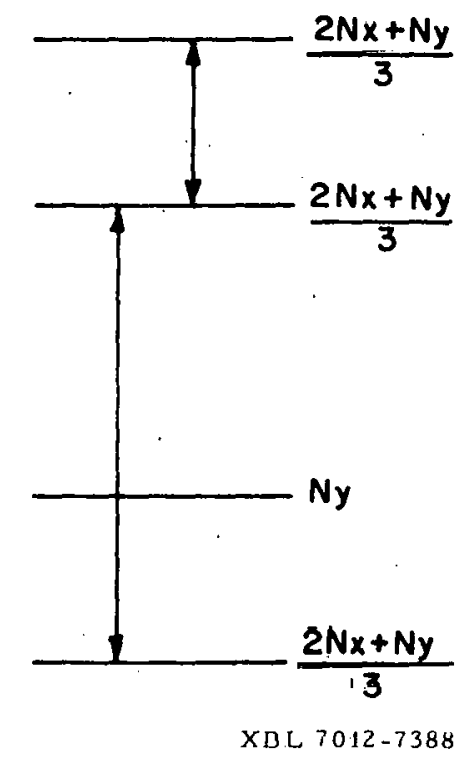

Figure 4

Population Change Predicted for ESR $(b \leftrightarrow d)$ and ENDOZ $(a \leftrightarrow b)$ Transitions 
each of the two triplet levels, and the ENDOR transition connects only two nuclear sublevels in one of the triplet levels.

As has already been discussed, the sensitivity of the optical detection technique is dependent on the various relaxation pathways from the triplet state. The same considerations apply in an ENDOR experiment. Since the sensitivity of the ENDOR experiment will be referenced to the sensitivity of the ESR experiment, the explicit dependence of the triplet state populations on the various rate constants need not be specified. For the system shown in Figure 4 , the phosphorescence intensity may then be written

$$
I_{0}=2\left(N_{x} K_{x}+N_{y} K_{y}\right)
$$

Upon saturation of the electron spin transition (b↔d), this becomes

$$
I_{s}=\left(\frac{3 N_{x}+N_{y}}{2}\right) K_{x}+\left(\frac{3 N_{y}+N_{x}}{2}\right) K_{y}
$$

with the change in intensity given by

$$
\Delta I=I_{s}-I_{0}=\frac{1}{2}\left(N_{x}-N_{y}\right)\left(K_{y}-K_{x}\right)
$$

If the ENDOR transition $(a \leftrightarrow b)$ is also saturated, the intensity is given by

$$
I_{E}=\frac{2}{3}\left[\left(2 N_{x}+N_{y}\right) K_{x}+\left(2 N_{y}+N_{x}\right) K_{y}\right]
$$

Since the ENDOR signal is detected by monitoring the change in intensity of the ESR transition, the signal strength is given by 


$$
\begin{aligned}
\Delta I_{E} & =I_{E}-I_{S} \\
& =\frac{1}{6}\left[\left(N_{x}-N_{y}\right)\left(K_{y}-K_{x}\right)\right]
\end{aligned}
$$

and the fractional change in intensity of the ESR signal upon saturation of the ENDOR transition is

$$
\delta I=\Delta I_{E} / \Delta I_{S}=\frac{1}{3}
$$

If the ENDOR transition ( $c \leftrightarrow d$ ) . is saturated instead of the transition from $(a \leftrightarrow b)$, the same expression is obtained for the change in intensity (Equations 42 and 43).

It is interesting to note from Equations 39 and 42 that the ESR signal and the ENDOR signal always affect the intensity of the phosphorescence in the same direction.

If the forbidden ESR transition from $(b \leftrightarrow c)$ is saturated and If lie twu ENDOR transitiuns ( $\Leftrightarrow$ ) and ( $c \leftrightarrow d$ ) occur at the same frequency, the change in phosphorescence intensity is given by

$$
\Delta I_{E}=\frac{I}{2}\left[\left(N_{x}-N_{y}\right)\left(K_{y}-K_{x}\right)\right]
$$

and the fractional change in intensity of the ESR signal is unity.

As a final note, if the ISR transition from ( $A \leftrightarrow c)$ and (b↔d) occur at the same frequency, the ENDOR transitions from $(a \leftrightarrow b)$ and $(c \leftrightarrow d)$ must also occur at the same frequency causing the change in intensity of the ESR signal to be twice as large (Equation 39).

$$
\Delta I=\left(N_{x}-N_{y}\right)\left(K_{y}-K_{x}\right)
$$

while the ENDOR transitions will not be observed since the populations of the nuclear sublevels are already equal. 
III. The Spin Hamiltonian

The observed magnetic resonance spectra of the excited triplet state of organic molecules in zero external magnetic field may be understood in terms of a Hamiltonian of the form,

$$
\mathrm{H}=\mathrm{H}_{\mathrm{SS}}+\mathrm{H}_{\mathrm{Q}}+\mathrm{H}_{\mathrm{HF}}
$$

where $\mathrm{H}_{\mathrm{SS}}$ is the spin-spin or zero field interaction between the two unpaired electrons, $\mathrm{H}_{Q}$ is the nuclear quadrupole interaction, and $\mathrm{H}_{\mathrm{HF}}$ is the nuclear electron hyperfine interaction.

This section is devoted to a review of. the explicit form of each term of the Hamiltonian and the resulting energy levels and transition probabilities.

A. $\quad \mathrm{H}_{\mathrm{SS}}--$ The Spin-Spin or Zero Field Splitting Hamiltonian

$\mathrm{H}_{\mathrm{SS}}$ is primarily due to the magnetic dipole-dipole interaction between the unpaired electrons in the excited triplet state. There can also be a contribution from the spin-orbit coupling between the lowest triplet and other excited states; however, the contribution from the interaction between other excited triplet states will shift the three levels equally, and may therefore be neglected. 56

If the radiative lifetime for fluorescence and phosphorescence is known, the magnitude of the spin-orbit contribution to the zero field splitting may be estimated by choosing a simple model in which the transition probability for phosphorescence is due only to the spinorbit coupling of one spiri sublevel with the nearest excited singlet state. In the framework of this model the transition probability for phosphorescence may be expressed as 


$$
\left.P_{P} \approx\left|<^{3} \psi_{1}\right| \text { er }\left.\right|^{2} \psi_{0}\right\rangle\left.\right|^{2}=\frac{1}{{ }^{\top} P}
$$

where $\mathrm{er}$ is the electron dipole moment transition operator, ${ }^{3} \psi_{1}$ is the first triplet state, ${ }^{1} \psi_{0}$ is the ground singlet state, and ${ }^{r_{P}}$ is the phosphorescence radiative lifetime. The wave function for the phosphorescent triplet state is actually a linear combination of the pure triplet state, which is spin forbidden for electric dipole radiation to the ground state, and an admixture of singlet character due to spin orbit coupling. $3 \Psi_{1}$ may therefore be represented as a linear combination of $3 \psi_{2}$ and ${ }^{1} \psi_{1}$ as

$$
{ }^{3} \Psi_{2}=C_{1} \cdot{ }^{3} \psi_{1}+C_{2}{ }^{2} \psi_{1}
$$

where ${ }^{3} \psi_{1}$ and ${ }^{1} \psi_{1}$ are the wave functions for the first excited singlet and triplet states respectively in the absence of spin-orbit coupling.

In organic molecules the spin orbit matrix element is generally sma $\perp$ so $C_{1} \approx 1$ while $C_{2}$ is given t'rom second order perturbation theory by;

$$
C_{2}=\frac{\left\langle{ }^{1} \psi_{1}\left|{ }^{\mathrm{H}_{\mathrm{SO}}}\right|^{3} \psi_{2}\right\rangle}{\left|{ }^{1} \mathbb{E}_{1}-{ }^{3} \mathrm{E}_{0}\right|}=\frac{\delta}{\left|{ }^{1} \mathbb{E}_{1}-{ }^{3} \mathbb{E}_{1}\right|} .
$$

where ${ }^{I_{E}} E_{1}$ is the energy of ${ }^{1} \psi_{1}$ and ${ }^{3} E_{0}$ is the energy of ${ }^{3} \psi_{1}$. The phosphorescence transition probability (Equation 1) may now be written

$$
\begin{aligned}
\frac{\perp}{{ }^{\tau_{P}}} & =\mid\left\langle\mathrm{C}_{1}{ }^{3} \psi_{0}+\mathrm{C}_{2}{ }^{1} \psi_{1}|e \overrightarrow{\mathrm{r}}|^{1} \psi_{0}>\left.\right|^{2}\right. \\
& =\left.\left.\mathrm{C}_{2}^{2}\left|<^{2} \psi_{2}\right| \mathrm{e} \overrightarrow{\mathrm{r}}\right|^{1} \psi_{0}\right|^{2}
\end{aligned}
$$

while the fluorescence transition probability is given by 


$$
\left.\mathrm{P}_{\mathrm{F}} \approx\left|<^{2} \psi_{2}\right| \mathrm{e} \overrightarrow{\mathrm{r}}\right|^{2} \psi_{\mathrm{O}}>\left.\right|^{2}=\frac{1}{\tau_{\mathrm{F}}}
$$

Substituting Equation 5 into Equation 4 , we have

$$
C_{2}^{2}=\frac{\tau_{F}}{\tau_{P}}=\frac{\delta^{2}}{\left|{ }^{2} E_{1}-{ }^{3} E_{0}\right|^{2}} \cdot
$$

Within the limits of the model, the spin-orbit matrix element is given by

$$
\delta=\left(\frac{{ }^{\tau} F}{{ }^{\top} P}\right)^{\frac{1}{2}}\left({ }^{2} E_{2}-{ }^{3} E_{O}\right)
$$

Also from second order perturbation theory the shift in energy of the triplet zero field level coupled to ${ }^{1} \psi_{i}$ may be written.

$$
\Delta=\frac{\varepsilon^{2}}{\left|{ }^{1} E_{1}-{ }^{3} E_{O}\right|}=\left(\frac{r_{F}}{r_{P}}\right)\left({ }^{2} E_{1}-{ }^{3} E_{O}\right) \text {. }
$$

As an example, for benzene, ${ }^{68} \tau_{P}=30 \mathrm{sec},,_{F}=3 \times 10^{-8} \mathrm{sec}$. , and $\left|{ }^{1} E_{1}-{ }^{3} E_{0}\right|=6000 \mathrm{~cm}^{-1}$. Therefore,

$$
\begin{aligned}
\Delta & =\frac{3 \times 10^{-8} \mathrm{sec} .}{30 \mathrm{sec}} \cdot\left(6000 \mathrm{~cm}^{-1}\right) \\
& =6 \times 10^{-5} \mathrm{~cm}^{-1}
\end{aligned}
$$

Compared to the measured zero field splittings of benzene of $0.1644 \mathrm{~cm}^{-1}$, $0.1516 \mathrm{~cm}^{-1}$, and $0.0128 \mathrm{~cm}^{-1}$, the spin-orbit coupling contribution to the zero field splitting is clearly negligible.

The adiition of a heavy atom will increase the spin orbit coupling 69,70 matrix element. An example of the magnitude of the effect is given by 
paradichlorobenzene for which $\tau_{P}^{70,71}=16 \mathrm{~ms} .,{ }_{F}=3 \times 10^{-8} \mathrm{sec}$, and $\left|{ }^{1} \dot{E}_{1}-{ }^{3} E_{0}\right|=7800 \mathrm{~cm}^{-1}$. Substituting these values into Equation 8 , we find that $\Delta=1.5 \times 10^{-2} \mathrm{~cm}^{=1}$. This is still small compared to the observed zero field splittings of $0.1787 \mathrm{~cm}^{-1}, 0.1201 \mathrm{~cm}^{-1}$, and 0.0584 $\mathrm{cm}^{-1}$. In addition, since we used the measured lifetime of the phosphorescence which includes both the radiative and non-radiative transition probabilities, the actual contribution of spin orbit coupling to the zero field splitting is certainly smaller.

For organic molecules in their excited triplet state, the splitting of the zero f'ield levels due to spin orbit coupling accounts for only a small percentage of the observed zero field splitting and therefore, we will consider only the magnetic dipole-dipole interaction in explaining the observed spectra.

The Hamiltonian for the magnetic dipole-dipole interaction between two unpaired olectrons may be written a.3'

$$
H_{S S}=g_{e}{ }^{2} \beta_{e}{ }^{2}\left\{\frac{S_{j} \cdot S_{2}}{r^{3}}-\frac{3\left(S_{j} \cdot r\right)\left(S_{2} \cdot r\right)}{r^{5}}\right\}
$$

where $g_{c}$ is the anomalous electron $g$ factor, which has been found to be basically isotropic for aromatic triplet states and equal to the free electron value of $2.0023 \varepsilon, \quad \beta_{e}$ is the Dohr magneton (eli/2me), and $x$ is the vector connecting the two electron spins $S_{1}$ and $S_{2}$.

The Hamiltonian is of the same form as any dipole-dipole interaction, and in the case of the interaction between the two triplet state electrons may be expressed as

$$
\mathrm{H}_{\mathrm{SS}}=\mathrm{S} \cdot \mathrm{D} \cdot \mathrm{S}
$$


which may be written in a Cartesian axis system as

$$
\begin{aligned}
H_{S S}= & D_{x x} S_{x}{ }^{2}+D_{x y} S_{x} D_{y}+D_{x z}{ }^{S} x_{z}+ \\
& D_{y x} S_{y} S_{x}+D_{x y} S_{y}{ }^{2}+D_{y z} S_{y} S_{z}+ \\
& D_{z x} S_{z} S_{x}+D_{z y} S_{z} S_{y}+D_{z z} S_{z}{ }^{2}
\end{aligned}
$$

The values of the $D_{i j}(i, j=x, y, z)$ are given by averages over the triplet state electronic wave function 57

$$
\begin{aligned}
& D_{x x}=\frac{1}{2} g_{e}{ }^{2}{ }^{2}\left\langle\frac{r^{2}-3 x^{2}}{r^{5}}\right\rangle \\
& D_{x y}=\frac{1}{2} g_{e}{ }^{2}{ }^{2}\left\langle\frac{-3 x y}{r^{5}}\right\rangle
\end{aligned}
$$

and so on. $D$ is a symetrical tensor. ( $D_{x y}=D_{y x}$, etc.); therefore, in the principal axis system which diagonalizes the zero field tensor, the Hamiltonian becomes

$$
H_{S S}=-X S_{x}^{2}-Y S_{y}^{2}-Z S_{z}^{2}
$$

where

$$
X=-D_{x x}, \quad Y=-D_{y y}, \text { and } Z=-D_{z z}
$$

Since the Hamiltonian satisfies LaPlace's equation, $X+Y+Z=0$, only two independent parameters are needed to describe the interaction. In conventional ESR the Hamiltonian in the principal axis system is usualiy rewritten by defining

$$
D=\frac{1}{2}(X+Y)-Z \text { and } E=-\frac{1}{2}(X-Y)
$$


with the axis convention that $|X| \leq|Y| \leq|Z|$. Therefore, the three components of the Hamiltonian are given by

$$
\begin{aligned}
& X=D / 3-E \\
& Y=D / 3+E \\
& Z=-2 / 3 D
\end{aligned}
$$

The Hamiltonian may be rewritten by substituting the definitions of $D$ and $E$ (Equation 14) into the Hamiltonian (Equation 13),

$$
\begin{aligned}
H_{S S} & =-(D / 3-E) S_{x}{ }^{2}-(D / 3+E) S_{y}{ }^{2}+2 / 3 D S_{z}{ }^{2} \\
& =D\left(2 / 3 S_{z}{ }^{2}-1 / 3\left(S_{x}{ }^{2}+S_{y}{ }^{2}\right)\right)+E\left(S_{x i}{ }^{2}-S_{y}{ }^{2}\right) .
\end{aligned}
$$

since $S_{x}{ }^{2}+S_{y}{ }^{2}=S^{2}-S_{z}{ }^{2}$,

we may write

$$
H_{S S}=D\left(S_{z}^{2}-1 / 3 S^{2}\right)+E\left(S_{x}^{2}-S_{y}^{2}\right)
$$

However, for the triplet state $S^{2}=2$; therefore,

$$
H_{S S}=D\left(S_{z}{ }^{2}-2 / 3\right)+E\left(E_{x}{ }^{2}-S_{y}{ }^{2}\right) \cdot
$$

The Hamiltonian may also be expressed in equivalent form using the raising and lowering operators as

$$
\mathrm{H}_{\mathrm{SS}}=\mathrm{D}\left(\mathrm{S}_{\mathrm{z}}{ }^{2}-2 / 3\right)+\mathrm{E} / 2\left(\mathrm{~S}+^{2}-\mathrm{S}_{-}{ }^{2}\right)
$$

The $D$ and $E$ parameters are defined by the average over the triplet state wave function

$$
\mathrm{D}=3 / 4 \mathrm{~g}_{\mathrm{e}}^{2} \beta^{2}\left\langle\frac{r^{2}-3 z^{2}}{r^{5}}\right\rangle
$$




$$
E=1 / 4 g_{e}^{2} \beta^{2}\left\langle\frac{3 y^{2}-3 x^{2}}{r^{5}}\right\rangle
$$

The Hamiltonian in matrix form with the basis states chosen to be eigenvalues of $\mathrm{Sz}$ may now be expressed as

$\mathrm{H}_{\mathrm{SS}}=$\begin{tabular}{c|c|c}
$|I\rangle$ & $|0\rangle$ & $|-1\rangle$ \\
\hline $\mathrm{D} / 3$ & 0 & $E$ \\
\hline $\mathrm{E}$ & $-2 / 3 \mathrm{D}$ & 0 \\
\hline & 0 & $D / 3$
\end{tabular}

This form is generally applicable and may be used with any interacting nuclear spin. In certain cases the simpler form may be used in which the basis states are eigenfunctions for both the Hamiltonian and $\mathrm{Sx}, \mathrm{Sy}$ and $\mathrm{Sz}$.

\begin{tabular}{|c|c|c|c|}
\hline \multirow{3}{*}{$\mathrm{H}_{\mathrm{SS}}=$} & $-X$ & 0 & 0 \\
\hline & 0 & $-Y$ & 0 \\
\hline & 0 & 0 & $-Z$ \\
\hline
\end{tabular}

where

$$
\begin{aligned}
& \mid x>=1 / \sqrt{2} \quad(|-1>-| 1>) \\
& \mid y>=i / \sqrt{2} \quad(|-1>+| 1>) \\
& \mid x>=10>
\end{aligned}
$$

This form of the Hamiltonian is directly related to the chosen axis system of the molecule and presents a clear picture of the orientational dependence of the energy. 
The resulting energy level diagram and nomenclature for both representations are shown in Figure 5 .

The usual selection rule in ESR of $\Delta S_{z}= \pm 1$ is not valid in zero magnetic field since the triplet sublevels are not eigenfuctions of $\mathrm{Sz}$. The nonvanishing matrix elements for magnetic dipole transitions between the triplet magnetic sublevels are given by

$$
\begin{aligned}
& P_{y \rightarrow z}=|<y| S_{x}|z>|^{2}=1 \\
& P_{x \rightarrow z}=|<x| S_{y}|z>|^{2}=1 \\
& P_{x \rightarrow y}=|<x| S_{z}|Y>|^{2}=1
\end{aligned}
$$

Therefore the three microwave transitions are polarized along the $x, y$ and $z$ directions as shown in Figure 2 .

\section{B. $\mathrm{H}_{\mathrm{Q}}-$ - The Iuc Leàr Quadrupole Hamiltonian}

A nucleus with a spin $\geq 1$ will have a non-spherical charge distribution and therefore an electric quadrupole moment. The quadrupole moment of the nucleus may be positive or negative depending on whether the charge distribution is elongated or flattened along the spin axis. Each allowed nuclear orientation along the spin axis will have associated with it a potential energy due to the surrounding electric field. In the case of a free molecule, the electric field is due to non-s electrons which produce a field gradient at the nucleus defined by

$$
V_{i, j}=\frac{\partial^{2} V}{\partial_{i}, \partial \cdot j} \quad(i, j=x, y, z)
$$

The Hamiltonian is derived by expanding the expression for the 


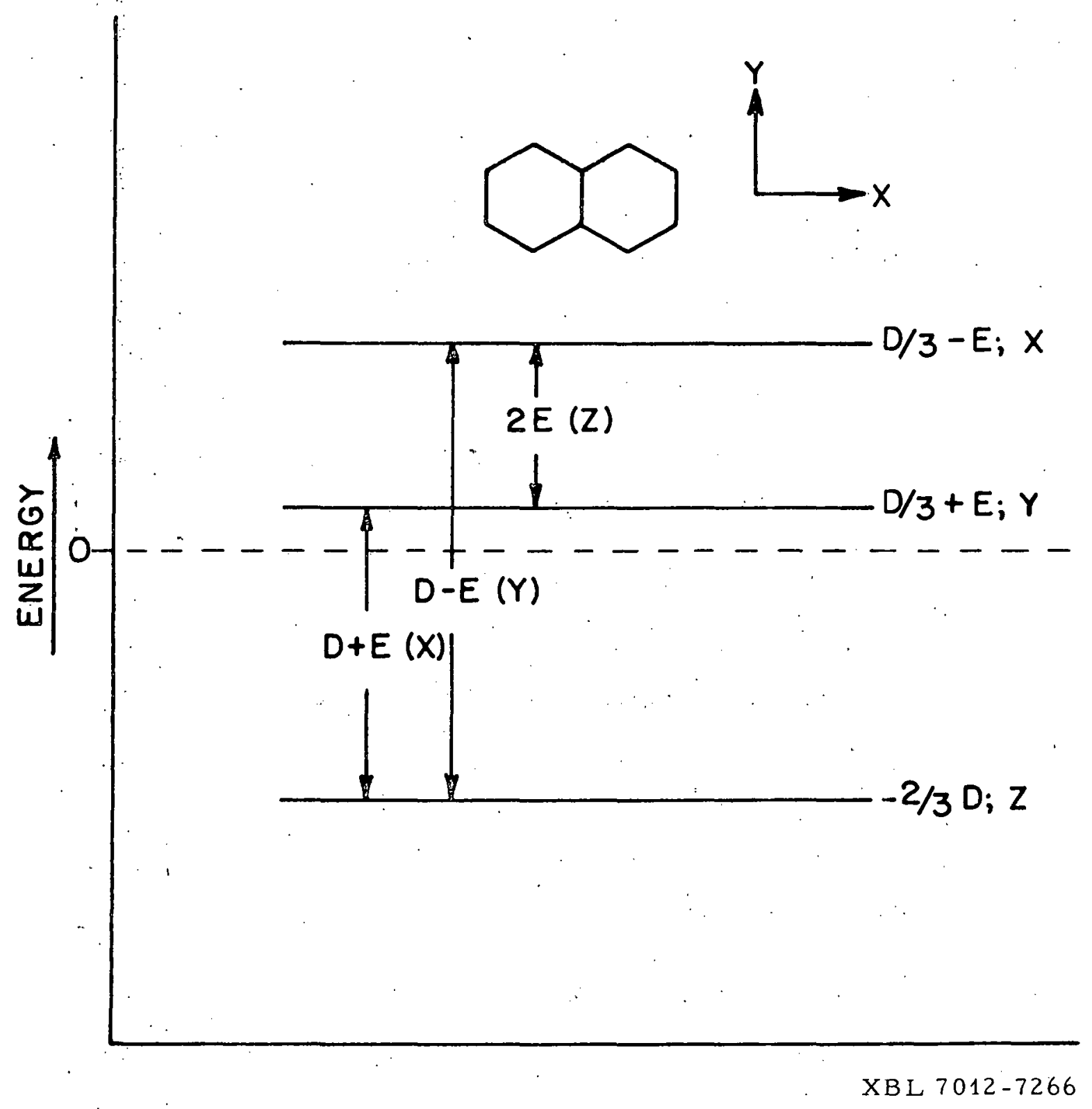

Figure 5

Energy Levesl and ESR Transitions for the Triplet State 
electrostatic potential energy of a nucleus of finite dimensions in the field of its surrounding electrons in terms of the nuclear multipole moments. The electrostatic dipole moment vanishes by symmetry and the first term in the expansion giving rise to deviations from a simple point charge interaction is the quadrupole term. The explicit derivation of the Hamiltonian is given in several sources and will not be repeated here. $58-62$.

In an arbitrary axio oystcm the Hamiltonian may be written as

$$
\begin{aligned}
H_{Q} & =B\left\{V_{z z}\left(3 I_{z}{ }^{2}-I^{2}\right)+\left(V_{z x}+i V_{z y}\right)\left(I_{-} I_{z}+I_{z} I_{-}\right)\right. \\
& +\left(V_{z x}-i V_{z y}\right)\left(I_{+} I_{z}+I_{z} I_{+}\right)+\left[I / 2\left(V_{x x}-V_{y y}\right)\right. \\
& \left.\left.+i V_{x y}\right] \cdot I_{+}^{2}+\left[I / 2\left(V_{x x}-V_{y y}\right)-i V_{x y}\right] I_{-}^{2}\right\}
\end{aligned}
$$

$$
\text { where } \begin{aligned}
B & =\frac{e Q}{4 \perp(2 \perp-1)} \\
e & =\text { the electron charge (esu) } \\
Q & =\text { the quadrupole mement }\left(\mathrm{cm}^{2}\right)
\end{aligned}
$$

and $I=$ the nuclear spin quantum number.

The Hamiltonian is a symmetric tensor and by transforming to an axis system such that $v_{i, j}=0$ for $i \neq \cdot j$, the Hamiltonian may be rewritten as:

$$
\dot{\mathrm{H}}_{\mathrm{Q}}=\mathrm{B}\left\{\mathrm{V}_{\mathrm{zz}}\left(3 \mathrm{I}_{\mathrm{z}}{ }^{2}-\mathrm{I}^{2}\right)+\left[I / \mathrm{P}\left(\mathrm{V}_{\mathrm{xx}}-\mathrm{V}_{\mathrm{yy}}\right)\left(I_{+^{2}}{ }^{2}+I_{-}{ }^{2}\right)\right]\right\}
$$

Since the Hamiltonian only includes interactions due to charges external to the nucleus, the LaPlace equation is satisfied and therefore:

$$
\mathrm{v}_{\mathrm{xx}}+\mathrm{v}_{\mathrm{yy}}+\mathrm{v}_{\mathrm{zz}}=0
$$


and it is only necessary to specify two independent parameters to describe the interaction. The conventional nomenclature in nuclear quadrupole resonance spectroscopy defines the field gradient, $q$, and the asymmetry parameter $\dot{\eta}$ by the relations

$$
\begin{aligned}
& \text { eq }=v_{z z} \\
& \eta \equiv \frac{v_{x x}-v_{y y}}{v_{z z}}
\end{aligned}
$$

with the convention

$$
\left|v_{x x}\right| \leq\left|v_{y y}\right| \leq\left|v_{z z}\right|
$$

The three components of the field gradient are then given by

$$
\begin{aligned}
\mathrm{v}_{\mathrm{zz}} & =\mathrm{eq} \\
\mathrm{v}_{\mathrm{xx}} & =\frac{-\mathrm{v}_{\mathrm{zz}}(1-\mathrm{n})}{2} \\
\text { and } \mathrm{v}_{\mathrm{yy}} & =\frac{-\mathrm{v}_{\mathrm{zz}}(1+\mathrm{n})}{2}
\end{aligned}
$$

The asymmetry parameter $\eta$ may take on values from 0 to 1 and in the case where $\eta=0$, the field gradient has axial symmetry and,

$$
\mathrm{v}_{\mathrm{xx}}=\mathrm{V}_{\mathrm{yy}}=-I / 2 \mathrm{v}_{\mathrm{zz}} \cdot
$$

When $\eta$ has its maximum value of 1 ,

$$
\begin{aligned}
& v_{x x}=0 \\
& v_{y y}=-v_{z z}
\end{aligned}
$$


The asymmetry perameter will vanish by symmetry whenever the nucleus is at a sight of three-fold or higher symmetry since this requires that $\mathrm{V}_{\mathrm{xx}}=\mathrm{V}_{\mathrm{yy}}$

The standard form of the Hamiltonian, Equation 28 , may now be written as

$$
\dot{H}_{Q}=A\left[\left(3 I_{Z}^{2}-I^{2}\right)+\eta / 2\left(I_{+}^{2}+I_{-}^{2}\right)\right]
$$

where $A=\frac{e^{2} a Q}{4 I(2 I-I)}$

This may also be written in the completely equivalent form

$$
H_{Q}=A\left[\left(3 I_{z}^{2}-I^{2}\right)+\eta\left(I_{x}^{2}-I_{y}^{2}\right)\right] \text {. }
$$

The Hamiltonian matrix therefore consists of diagonal terms and off diagonal terms connecting states differing in $I_{z}$ by \pm 2 .

At this point we will consider the explicit form of the Hamiltonian matrix for $I=I$ and $I=3 / 2$ since interactions due to both spins were observed in the course of this work.

The Hamiltonian for an $I=I$ nucleus may be expressed in matrix form as

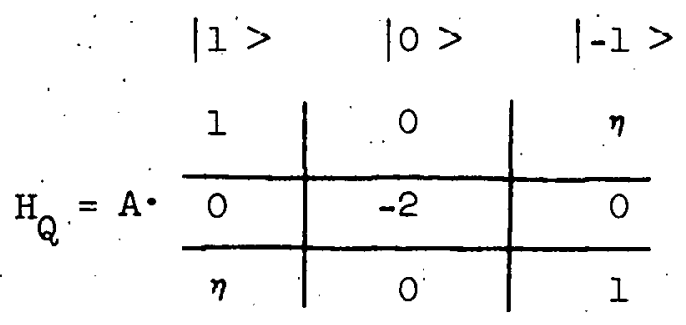

where $A=\frac{e^{2} q Q}{4}$

A more convenient form of the Hamiltonian is obtained by transforming the Hamiltonian to the representation in which the energy is diagonal. In this 
representation the Hamiltonian is in the same form as the spin-spin Hamiltonian, Equation 13 , and in matrix form, may be written;

\begin{tabular}{c|c|c}
\multicolumn{1}{c}{$|\mathrm{X}\rangle$} & $|\mathrm{Y}\rangle$ & $|\mathrm{Z}\rangle$ \\
$\mathrm{H}_{\mathrm{Q}}=\mathrm{A} \cdot \mathrm{-X}$ & 0 & 0 \\
\hline$\therefore$ & $-\mathrm{Y}$ & 0 \\
\hline 0 & 0 & $-\mathrm{Z}$
\end{tabular}

The energy is given by

$$
\begin{aligned}
& X=-A(I+\eta) \\
& Y=-A(I-\eta) \\
& Z=-2 A
\end{aligned}
$$

and the eigenstates are

$$
\begin{aligned}
& \mid X>=(I / \sqrt{2})(|1>+|-1>) \\
& \mid Y>=(i / \sqrt{2})(|1>-|-1>) \\
& |Z>=| 0>.
\end{aligned}
$$

This form of the Hamiltonian is particularly convenient since it may be written in terms of the nuclear angular momentum operators as

$$
H_{Q}=-x I_{x}^{2}-y I_{y}^{2}-z I_{z}^{2}
$$

which is in the same form as the zero field Hamiltonian.

In Figure $6 \mathrm{a}$, the energies and polarizations of the pure nuclear quadrupole transitions, for a spin 1 nucleus, are given. The three magnet1c dipole transitions are polarized along the $X, Y$ and $Z$ axis as are the zero field transitions. 
$-46-$

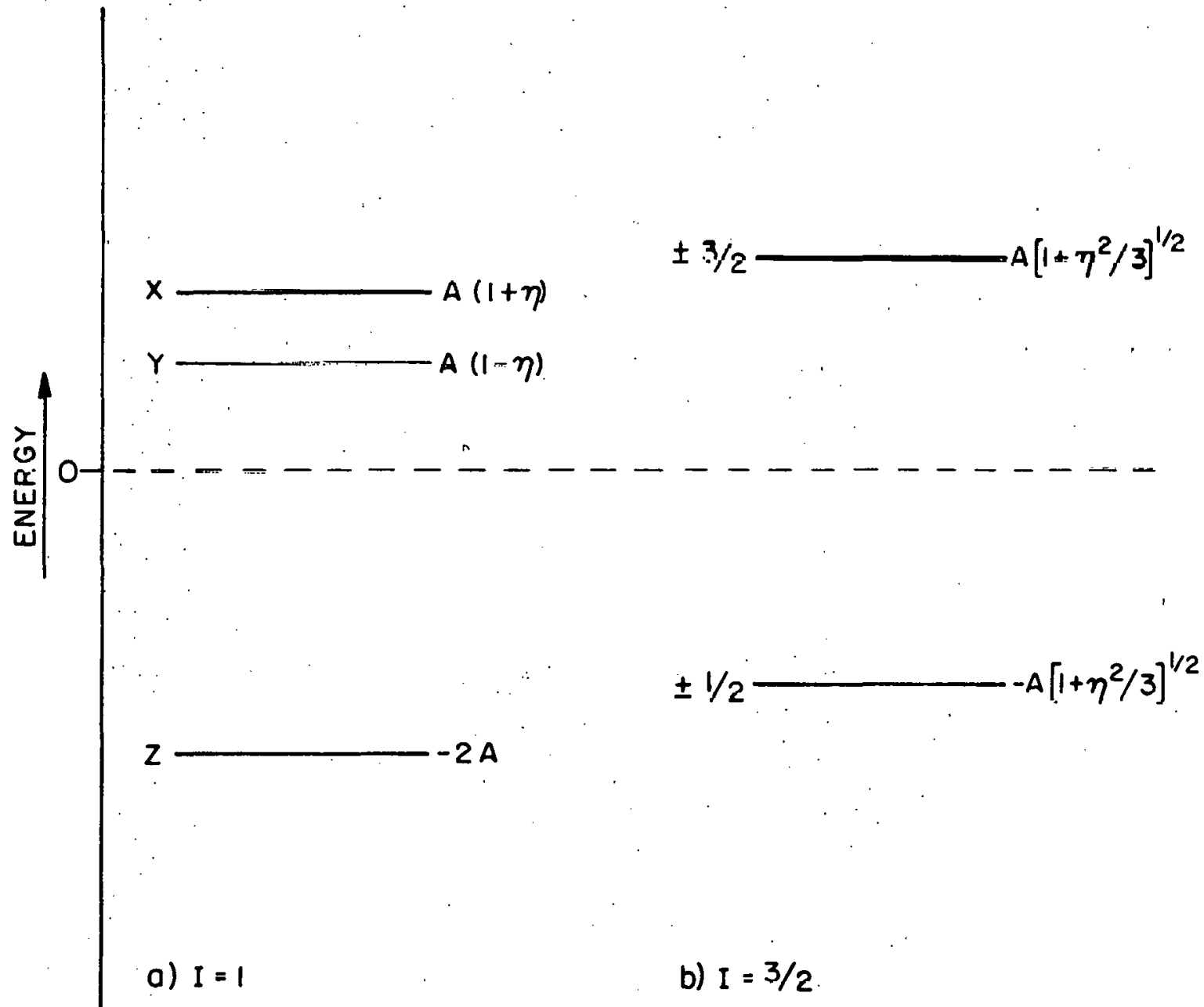

XBL $7012-7265$

F1gure 6

Nuclear Quadrupole Energy Level Diagram for a) $I=I$

and b) $I=3 / 2$ rhaclear Spins 
The Hamiltonian matrix for $I=3 / 2$ may be written as

\begin{tabular}{|c|c|c|c|c|}
\hline & 1 & 0 & $\eta / \sqrt{3}$ & 0 \\
\hline & O & -1 & 0 & $7 / \sqrt{3}$ \\
\hline $\mathrm{H}_{\mathrm{Q}}=\frac{\mathrm{e}^{c} \mathrm{qQ}}{4}$ & $\eta / \sqrt{3}$ & 0 & -1 & 0 \\
\hline 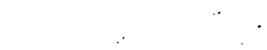 & 0 & $\eta / \sqrt{3}$ & 0 & 1 \\
\hline
\end{tabular}

The matrix may be rewritten as two separate $2 \times 2$ matrices by. rearranging the order of the basis states as

\begin{tabular}{c|c|c|c|c}
$|3 / 2\rangle$ & $\mid-1 / 2>$ & $1 / 2\rangle$ & $|-3 / 2\rangle$ \\
$H_{Q}=\frac{e^{2} q Q}{4}$ & $\eta / \sqrt{3}$ & 0 & 0 \\
\hline$\eta / \sqrt{3}$ & -1 & 0 & 0 \\
\hline 0 & 0 & -1 & $\eta / \sqrt{3}$ \\
\hline 0 & 0 & $\eta / \sqrt{3}$ & $\ddots 1$
\end{tabular}

The eigenvalues of the Hamiltonian may now be obtained by diagonalizing each of the $2 \times 2$ matrices with the result that there are only two energy levels, both of which are doubly degenerate (see Figure 6b).

$$
\begin{aligned}
& E_{ \pm 3 / 2}=\frac{e^{2} q Q}{4}(1+\eta / 3)^{1 / 2} \\
& E_{ \pm} I / 2-\frac{-e^{2} q Q}{4}(1+\eta / 3)^{1 / 2}
\end{aligned}
$$

The eigenstates are

$$
\begin{aligned}
& \left|3 / 2>^{\prime}=a\right| 3 / 2>+b \mid-1 / 2> \\
& \left|-1 / 2>^{\prime}=a\right|-1 / 2>-b \mid 3 / 2> \\
& |1 / 2>\prime=a| 1 / 2>-b \mid-3 / 2> \\
& \left|-3 / 2>^{\prime}=a\right|-3 / 2>+b \mid 1 / 2>
\end{aligned}
$$


where

and

$$
\begin{aligned}
& a=\frac{1+\sqrt{1+x^{2}}}{\left[2\left(1+x^{2}+\sqrt{1+x^{2}}\right)\right]^{1 / 2}} \\
& b=x /\left[2\left(1+x^{2}+\sqrt{1+x^{2}}\right]^{1 / 2}\right.
\end{aligned}
$$

In contrast to a nucleus with spin $I=1$, we cannot determine both $\mathrm{e}^{2} \mathrm{qQ}$ and $\eta$ by measuring only the transition energy since the levels are twofold degenerate. It is therefore necessary to apply a perturbation such as a Zeeman field to romove the degeneracy of the \pm levels in order to completely measure the nuclear quadrupole interaction: It should be noted, however, that the transition frequency is not particularly sensitive to $\eta$. The assumption that $\eta=0$ and therefore that the transition energy is equal to (1./?) $e^{2} q Q$ will produce only a small error for small values of $\eta$ as shown in Figure 7. Furthermore, an oscillating magnetic field along the $\mathrm{Z}$ axis will not induce magnetic dipole transitions between the $\pm 3 / 2$ and $\pm 1 / 2$ nuclear levels if $\eta=0$.

\section{C. $\mathrm{H}_{\mathrm{HP}}=$ - The Nuclear Electron Hyperfine Interaction}

A nucleus with a spin $\geq 1 / 2$, like an electron, will have a magnetic moment. The interaction of this nuclear magnetic moment, with the electron magnetic moment, will lead to both an anisotropic dipole-dipole interaction and the Fermi contact interaction due to a finite electron spin density at the nucleus.

The component of the hyperfine interaction, due to the interaction of the nuclear and electron magnetic moments, is entirely analogous to the 
$-49-$

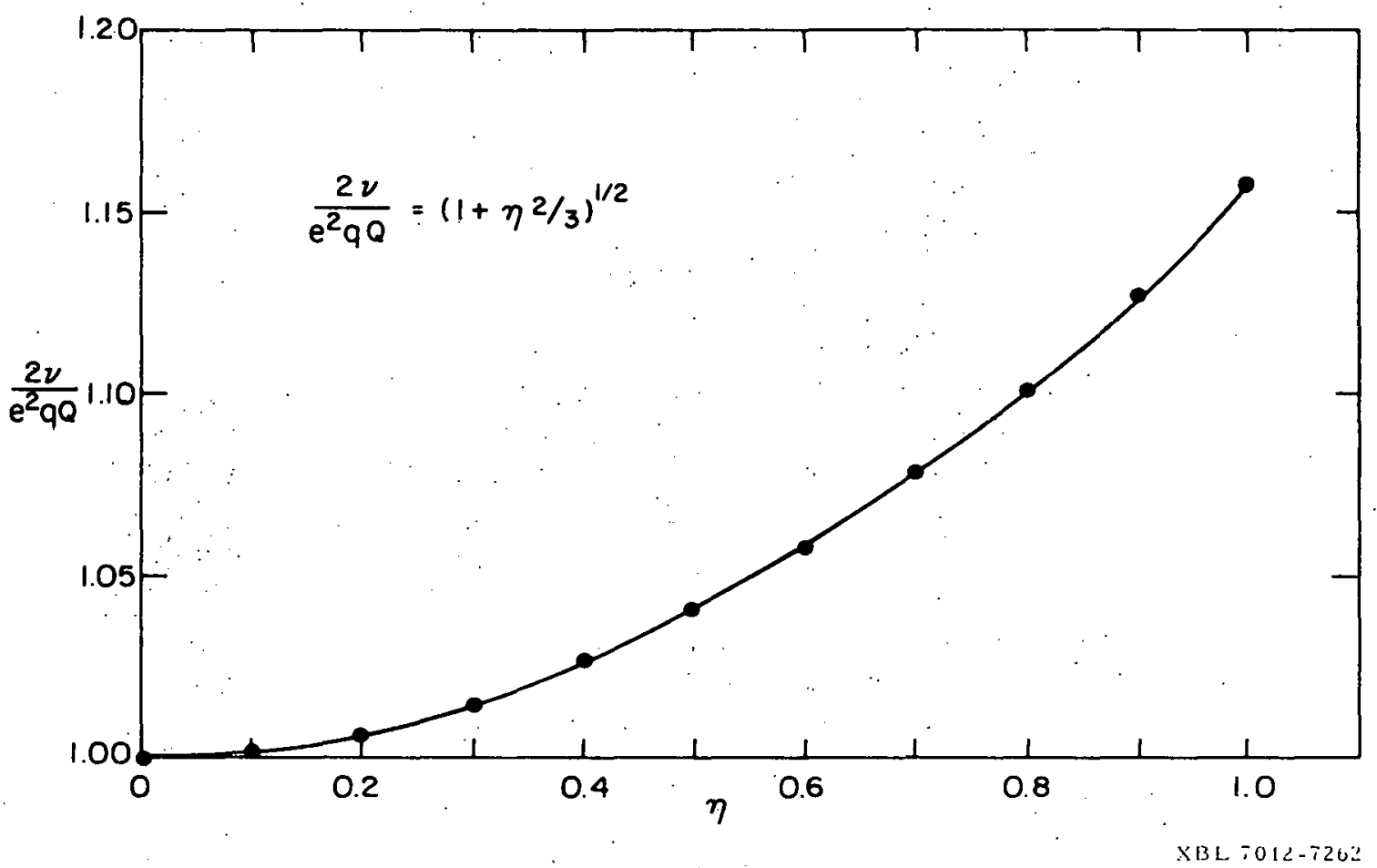

Figure 7

Plot of the Ratio of the Observed NRR Transition Frequency to the Nuclear Quadrupole Coupling Constant versus the Asymetry Parameter 
zero field Hamiltonian with the replacement of one of the electron spins with a nuclear spin and the appropriate change of constants. The Hamiltonian may be written as

$$
\dot{H}_{H F}^{D D}=-g_{e} \beta_{e} g_{n} \beta_{n}\left[\frac{I \cdot S}{r^{3}}-\frac{3(I \cdot r)(S \cdot r)}{r^{5}}\right]
$$

where $g_{n}$ is the nuclear $g$ factor and $\beta_{n}$ is the nuclear magneton.

Since this is identical in form to Equation 9 for the zero field Hamiltonian, Equation 47 may be expressed as :

$$
\mathrm{H}_{\mathrm{HF}}^{\mathrm{DD}}=\mathrm{S} \cdot \mathrm{A} \cdot \mathrm{I}
$$

which may be expanded in tha same manner as Equation 11 . The A matrix is symmetric and therefore, in its principle axis system, may be written as,

$$
\mathrm{H}_{\mathrm{HF}}^{\mathrm{DD}}=\mathrm{A}_{\mathrm{xx}} \mathrm{S}_{\mathrm{x}} I_{x}+\mathrm{A}_{\mathrm{yyy}} \mathrm{S}_{\mathrm{y}} I_{y}+A_{z z} S_{z} I_{z}
$$

where the hyperfine elements are given by the average over the spatial distribution of the unpaired spins.

$$
A_{x x}=-g_{e} e_{n} \beta_{e} \beta_{n}\left\langle\frac{r^{2}-3 x^{2}}{r^{5}}\right\rangle
$$

where $x=x, y, z$.

The LaPlace equation is again satisfied and therefore,

$$
A_{x x}+A_{y y}+A_{z z}=0
$$

The unpaired spin density at the nucleus will produce an additional 
contribution to the hyperfine Hamiltonian called the Fermi contact term. This will arise only from spin density in $s$ orbitals since the other orbitals have a vanishing probability of being at the nucleus. The Fermi contact contribution is usualiy considered to be isotropic and may therefore be written as

$$
H_{H F}^{F}=: C\left(S_{x} I_{x}+S_{y} I_{y}+S_{z} I_{z}\right)
$$

where

$$
C=(8 \pi / 3) \gamma_{e} \gamma_{n} n^{2}\left|\cdot \psi_{s}(0)\right|^{2}
$$

and $\left|\psi_{S}(0)\right|^{2}$ is the $s$ electron spin density at the nucleus.

The total hyperfine Hamiltonian may now be written as

$$
H_{H F}=A_{x x}^{\prime} S_{x} I_{x}+A_{y y}^{\prime} S_{y} I_{y}+A_{z z}^{\prime} S_{z} I_{z}
$$

where

$$
A_{x x}^{\prime}=A_{X x}+\dot{C}, \text { etc. }
$$

Therefore, if the three components of the total hyperfine Hamiltonian are measured, the contribution due to the anisotropic and isotropic components can be separated; however, the absolute signs will not generally be obtained. It should be pointed out that since the nuclei in which we are interested also have quadrupole moments, the Fermi contact term will not be strictly isotropic since the nuclei are distorted, and consequently, the dipole-dipole and contact terms are not completely separable. 
D. The Total Hamiltonian, Energy Levels and Transition Probabilities

In this section the total Hamiltonian for two molecules which are examples of the triplet state electrons interacting with an $\mathrm{I}=1$ and an $I=3 / 2$ nuclear spin will be considered. In order to simplify the discussion we will make the following assumptions for both cases:

1) The principal axis system of $\mathrm{H}_{\mathrm{SS}}, \mathrm{H}_{\mathrm{Q}}$ and $\mathrm{H}_{\mathrm{HF}}$ are coincident,

2). Only the out-of-plane component of the hyperfine Hamiltonian need be considered, and

3). The hyperfine interaction due to protons may be neglected.

Assumptions 1 and 2 can be, in many cases, justified on the basis of the single crystal ESR spectra, 43 and

assumption 3 on the fact that resolved proton hyperfine splitting has not been observed in zero field ESR.

Example $\# \perp, I=1$

An example of a molecule which is characterized by the interaction of one $(I=1)$ nuclear spin with the triplet electrons is the $\pi \pi^{*}$ state of quinoline (l-azanaphthalene). The spin Hamiltonian for this molecule may be written for the axis system given in Fig. 8 as 22,24

$$
\mathrm{H}=\mathrm{H}_{\mathrm{SS}}+\mathrm{H}_{\mathrm{Q}}+\mathrm{H}_{\mathrm{HF}}
$$

where

$$
\begin{aligned}
& H_{Z F}=-X S_{X}{ }^{2}-Y S_{y}{ }^{2}-{Z S_{z}{ }^{2}}^{H_{Q}=-X I_{X}{ }^{2}-y I_{y}{ }^{2}-Z I_{z}{ }^{2}}
\end{aligned}
$$

and

$$
\mathrm{H}_{\mathrm{HF}}=\mathrm{A}_{\mathrm{xx}} \mathrm{S}_{\mathrm{x}} \mathrm{I}_{\mathrm{x}}
$$



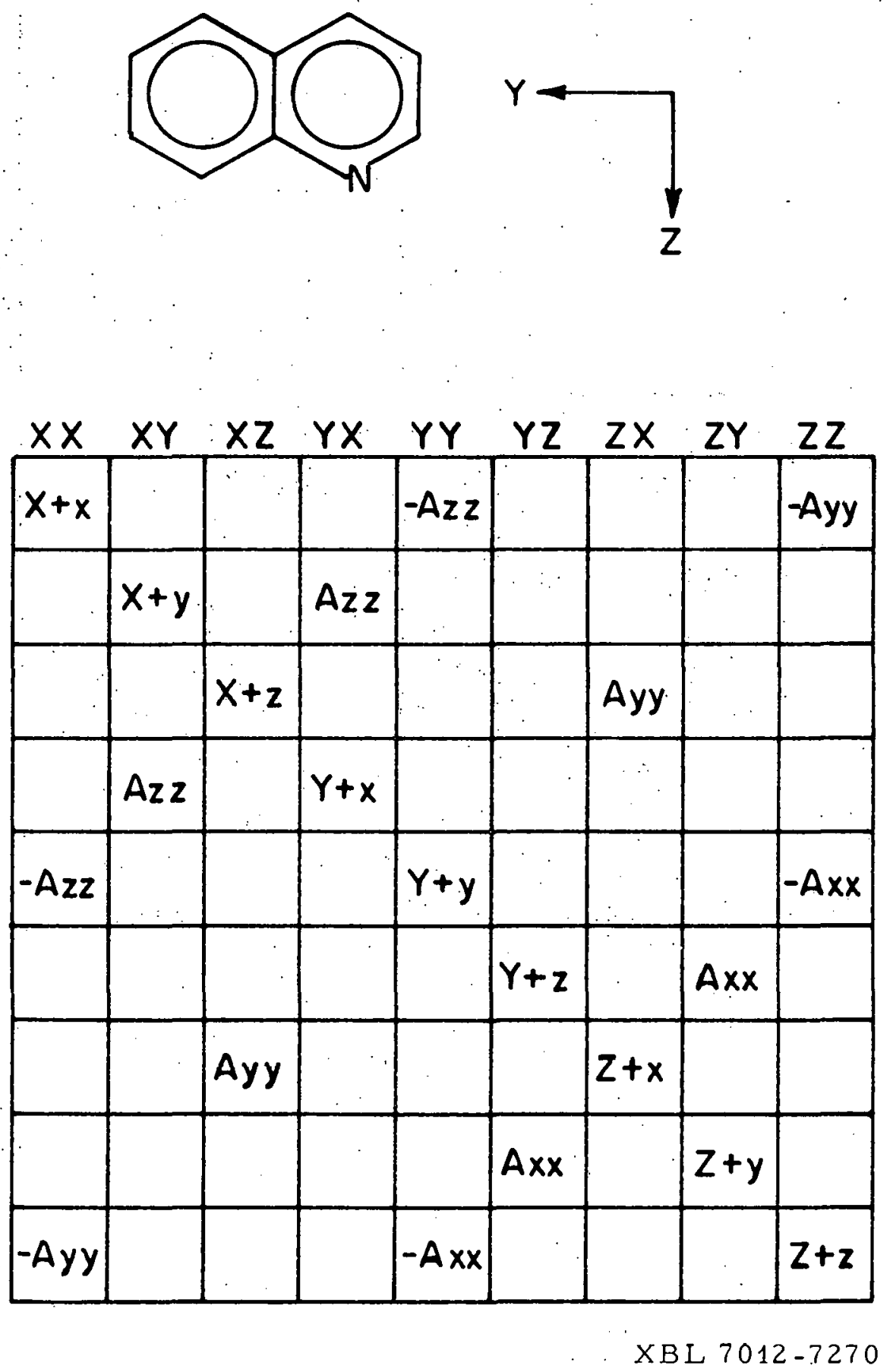

Figure 8

Hamiltonian Matrix for Triplet Plus One $I=1$ Nuclear Spin 
We will use for the basis states the product functions $|\mu \nu\rangle=\tau_{\mu}$ $x_{\nu}$, which form a set of eigenfunctions that diagonalize $\mathrm{H}_{\mathrm{SS}}$ and $\mathrm{H}_{\mathrm{Q}^{*}}{ }^{\boldsymbol{r}_{\mu}}$ and $x_{\nu}$ are the electron and nuclear spin function while $\mu$ and $\nu$ correspond to $x, y$ and $z$.

The complete Hamiltonian is a $9 \times 9$ matrix as shown in Figure 8, where we have also included the in-plane hyperfire elemerls for completeness. Since we are only considering the $A_{x x}$ element of the hyperfine interaction, wc can oolve the energies exast.ly hy dia.gnnalizing only two $2 \mathrm{x} \cdot 2$ matrices. However, a satisfactory solution is obtained by second order perturbation thcroy. $\Lambda o$ is shown in Figure 9 , the pnergy of the states $|\mathrm{Zz}\rangle$ and $|\mathrm{Zy}\rangle$ are shifted by an amount $\beta$, where

$$
\beta=\frac{A x{ }^{2}}{E_{y}-E_{z}}
$$

while the states $|\mathrm{Yz}\rangle$ and $|\mathrm{Yy}\rangle$ are shifted by an amount $\beta$.

Th our axis system the triplet state energy levels would be ordered $\mathrm{Z}>\mathrm{Y}>\mathrm{X}$ and the nuclear quadrupole energy levels ordered $\mathrm{x}>\mathrm{z}>\mathrm{y}$. The eigenvectors of the states which are coupled by $A_{x x}$ are

$$
\begin{aligned}
& \left|\mathrm{Z}_{\mathrm{z}}>^{\prime}=(1-\beta)\right| \mathrm{Zz}>-\beta \mid \mathrm{Yy}> \\
& \left|\mathrm{Zy}>^{\prime}=(1-\beta)\right| \mathrm{Zy}>-\beta \mid \mathrm{Yz}_{\mathrm{z}}> \\
& \left|\mathrm{Y}_{1}>^{\prime}=(1-\beta)\right| \mathrm{Yz}>+\beta \mid \mathrm{Zy}> \\
& \left|\mathrm{Yy}_{\mathrm{y}}>^{\prime}=(1-\beta)\right| \mathrm{Yy}>+\beta \mid \mathrm{Zz}>.
\end{aligned}
$$

The probability for microwave transitions between the triplet state magnetic sublevels is given by

$$
I \approx\left|<\mu_{2} \nu_{2}\right| H_{R F(t)}\left|\mu_{2} \nu_{2}>\right|^{2}
$$




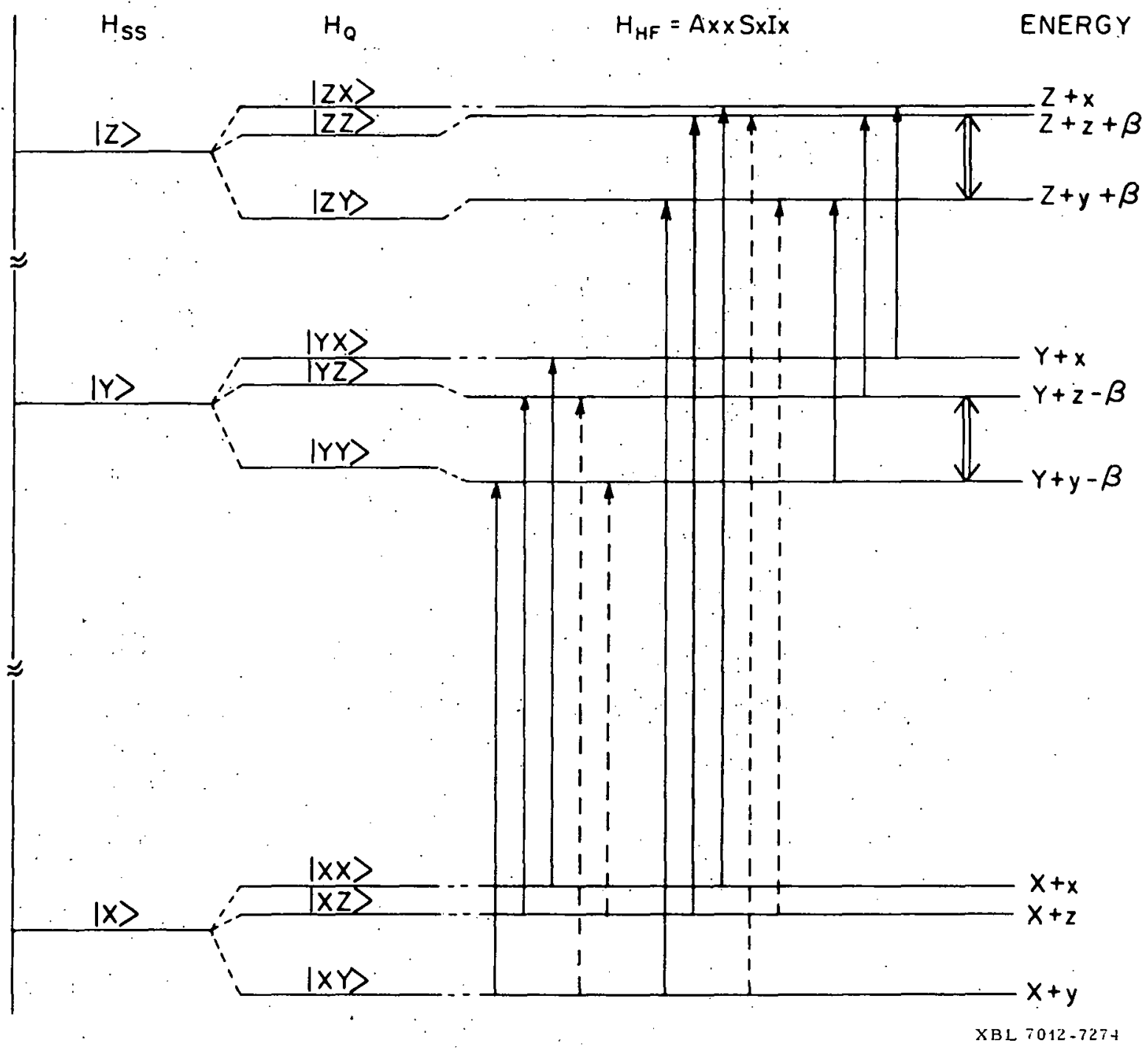

Figure 9

Energy Level Diagram for the 'l'riplet and One $I=I$ Nuclear Spin Considering only the $A_{x x}$ Hyperfine Component 
where $\mathrm{H}_{\mathrm{RF}}(t)$ is the magnetic dipole transition operator defined by;

$$
H_{R F(t)}=H_{1}(t) \hbar\left(\gamma_{n} \cdot I+\gamma_{e} \cdot S\right)
$$

and $\mathrm{H}_{2}(t)$ is the magnitude of the time dependent magnetic field, $\gamma_{n}$ is the nuclear gyromagnetic ratio $\left(\frac{g_{n} \beta_{n}}{n}\right)$ and $\gamma_{e}$ is the electron gyromagnetic $\operatorname{ratio}\left(\frac{g_{e} \beta_{e}}{\hbar}\right)$.

The electron spin magnetic dipole transition operator will connect states with $\mu_{2} \neq \mu_{2}$ and $\nu_{2}=\nu_{2}$, while the nuclear spin operator wili connect states with $\dot{\mu}_{1}=\mu_{2}$ and $\nu_{1} \neq \nu_{2}$. However, the mixing of the basis function by $A_{x x}$ will allow the observation of "forbidden" simultaneous electron and nuclear transitions. This is clearly shown by considering the transition from $|\mathrm{Xz}\rangle$ ! to $|\mathrm{Yy}\rangle$ '. The intensity of the transition is given by

$$
\begin{aligned}
& I \approx|<\mathrm{Xz}| \gamma_{\mathrm{e}^{\mathrm{H}}}(\mathrm{t})\left|\left[(\mathrm{I}-\beta)|\mathrm{Yy}>+\beta| \mathrm{Z}_{\mathrm{z}}>\right]\right|^{2} \\
& \mathrm{I} \approx \beta^{2} \gamma_{\mathrm{e}}^{2} \mathrm{H}_{1}(\mathrm{t})^{2}
\end{aligned}
$$

It should be noted that it is necessary to have a hyperfine interaction in order to observe the nuclear quadrupole satellites since the hyperfine term is the only method of coupling the electron and nuclear Hamiltonians.

In Figure 10, the spectra expected for the three zero field transitions are shown in terms of the components of the total Hamiltonian. It is clear that the separation of the quadrupole satellites for both the $\tau_{\mathrm{x}} \rightarrow \tau_{\mathrm{z}}$ and $\tau_{\mathrm{x}} \rightarrow \tau_{\mathrm{y}}$ transitions are separated by $2(\mathrm{z}-\mathrm{y})$ and therefore only one of the three possible nuclear quadrupole transitions equal 
$-57-$

a)

$$
\begin{aligned}
& \tau_{x} \rightarrow \tau_{z} \\
& \nu_{0}=z-x
\end{aligned}
$$

b)

$$
\begin{aligned}
& \tau_{x} \rightarrow \tau_{y} \\
& \nu_{0}=Y-X
\end{aligned}
$$

c)

$$
\begin{aligned}
& \tau \cdot y \rightarrow \tau z \\
& \nu_{0}=Z-Y
\end{aligned}
$$

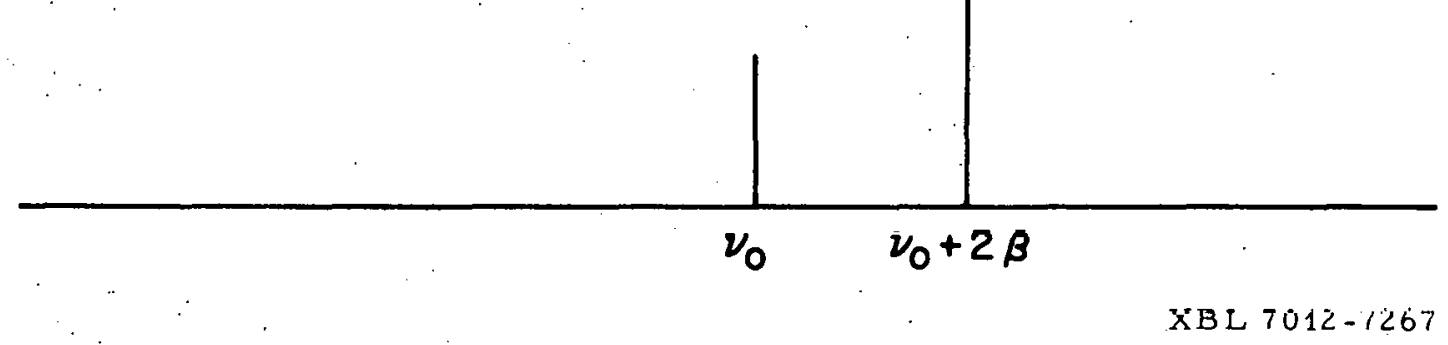

Figure 10 .

ODMR Spectra Predicted for the Energy Level Diagram Shown in Figure 9 
to $(3 / 4) e^{2} q Q(1-\eta / 3)$ is observed. ${ }^{24}$ The value of the hyperfine coupling constant $A_{X X}$ is easily obtained from the separation of the two allowed components of each of the three transitions. If we had chosen to use $A_{y y}$ or $A_{z z}$ as the only hyperfine interaction instead of $A_{x x}$, the spectra would be the same as that shown in Figure 10 if a cyclic perturbation is applied to our labeling.

Although in this simple example all the parameters in the Hamiltonian can be determined from the three zero t'ield transitions, in practice this is usually not the case. This can be due to such problems as poor resolution of the spectra or the failure to include enough terms in the Hamiltonian to adequately describe the interactions. Therefore, it is usually advantageous to also perform an electron nuclear double resonance (ENDOR) experiment to improve the resolution and confirm the assignment of the spectra. The ENDOR transitions are shown in Figure 9 by lise duuble arruws. However, speetra have been assigned incorrcctly because of a failure to consider the proper form of the magnetic dipole transition operator. 26 Therefore, let us consider, as an example, the intensity of the ENDOR transition from $\mid \mathrm{Yy}>$ ' to $\mid \mathrm{Yz}_{\mathrm{z}}>^{\prime}$;

$\mathrm{I} \approx|[(\mathrm{I}-\beta)<\mathrm{Yy}|+\beta<\mathrm{Zy}|]| \mathrm{H}_{\mathrm{RF}(\mathrm{t})}||(\mathrm{I}-\beta)|\mathrm{Yz}>+\beta| \mathrm{Zy}>||^{2}$
$\mathrm{I} \approx\left[(1-\beta)^{2} \gamma_{\mathrm{n}} \mathrm{H}_{1}+2 \beta(\mathrm{I}-\beta) \gamma_{\mathrm{e}} \mathrm{H}_{1}+\beta^{2} \gamma_{\mathrm{n}} \mathrm{H}_{1}\right]^{2}$.

Since $\mathrm{H}_{2}$ is a constant, we will drop it and may now write

$$
\begin{aligned}
& I \approx 4 \gamma_{e}^{2}\left[\beta^{2}(1-\beta)\right]+4 \gamma_{\mathrm{e}} \gamma_{\mathrm{n}}\left[\beta(1-\beta)^{3}+\beta^{3}(1-\beta)\right] \\
& \quad+\gamma_{\mathrm{n}}^{2}\left[(1-\beta)^{4}+\beta^{4}+2 \beta^{2}(1-\beta)^{2}\right]
\end{aligned}
$$


Since $\beta$ is usually on the order of $1 \times 10^{-2}$ for $\pi \pi^{*}$ triplets, we can reasonably approximate $\mathrm{Eq}$. 11 by

$$
I \approx 4 \beta^{2} \gamma_{e}^{2}+4 \beta \gamma_{e} \gamma_{n}+\gamma_{n}^{2}
$$

In contrast, if there were no hyperfine coupling as in the $\tau_{x}$ manifold in our example, the intensity would be given by

$$
I \approx Y_{n}^{2}
$$

Therefore, the ratio of the intensity of the ENDOR transitions due to the electron magnetic dipole operator to those due to the nuclear magnetic dipole operator is approximately $4 \beta^{2} \gamma_{e}^{2} / \gamma_{n}^{2}$ and therefore, unless $4 B^{2} / \gamma_{n}^{2}$ is greater than $I / \gamma_{e}^{2}$, the electron dipole moment transition operator will be the major source of the intensity in ENDOR transitions.

An example, for ${ }^{14} \mathrm{~N}$ the ratio of $\gamma_{e} / \gamma_{n}=8.6 \times 10^{6}$ and therefore, $B$ must be less than $1.57 \times 10^{-3}$ for the nuclear magnetic dipole transition operator to be comparable to the electron magnetic dipole transition operator in producing intensity in the ENDOR transitions. For a typical separation of $\tau_{z}-\tau_{y}$ of $1000 \mathrm{MHz}$ this would correspond to an extremely small hyperfine element, $A_{x x}$ of only $1.5 \mathrm{HMz}$, which is much smaller than any out-of-plane hyperfine elements reported for aza-aromatics

Example \#2, I=3/2

An example of a molecule characterized by the interaction of the unpaired electrons with one $I=3 / 2$ nuclear spin is the $\pi \pi^{*}$ state of chlorobenzene. The ESR spectrum in this case is somewhat more difficult to calculate due to the lack of a basis set that diagonalizes both $\mathrm{H}_{\mathrm{SS}}$ and $\mathrm{H}_{\mathrm{Q}}$ for an arbitrary value of the assymetry parameter. This provlem may be avoided by making the reasonable assumption that the chlorine assymetry 
parameter equals zero. In this case both $\mathrm{H}_{\mathrm{SS}}$ and $\mathrm{H}_{\dot{Q}}$ are diagonal with the basis set $\mid \mu v>=\tau_{\mu} \chi_{\nu}$ where $\tau$ and $\chi$ represent the electron and nuclear spin functions respectively, while $\mu$ corresponds to $X, Y$, and $\mathrm{Z}$ and $v$ to $3 / 2,1 / 2,-1 / 2$ and $-3 / 2$.

For this example, again only the out-of-plane component of the hyperfine tensor $\left(A_{x x}\right)$ will be considered and therefore the spin Hamiltonian may be written

$$
H=H_{S S}+\dot{H}_{\dot{Q}}+H_{H F}
$$

Since only the $A_{x x}$ component of the hyperfine tensor is being considered, $\mathrm{H}_{\mathrm{H} F}$ may be written as:

$$
\mathrm{H}_{\mathrm{HF}}=1 / 2\left[\mathrm{~A}_{\mathrm{Xx}} \mathrm{S}_{\mathrm{x}}\left(\mathrm{I}_{+}+\mathrm{I}_{-}\right)\right]
$$

Therefore, the basis states in the $\tau_{z}$ manifold are coupled to those in the $\tau_{y}$ manifold that differ in their nuclear spin quantum number ( $I_{z}$ ) by \pm 1 .

In order to make the Hamlltonlan: real it is cunvenient to redefine the electron spin basis states as,

$$
\begin{aligned}
& \left|\tau_{\mathrm{x}}\right\rangle=(1 / \sqrt{2})(|-1>-|+1>) \\
& \left|\tau_{\mathrm{y}}\right\rangle=(1 / \sqrt{2})(|-1>+|+1>) \\
& \left|\tau_{\mathrm{z}}\right\rangle=\mid 0>
\end{aligned}
$$

The complete spin Hamiltonian including both the $A_{x x}$ and $A_{g z}$ hyperfine elements is shown in Fig. 11. The hyperfine Hamiltonian may be treated as a perturbation of the eigenstates of $\mathrm{H}_{S S}$ and $\mathrm{H}_{Q}$ by nun-degenerate perturbation theory since the degenerate nuclear levels are not coupled by the same hyperfine element. 


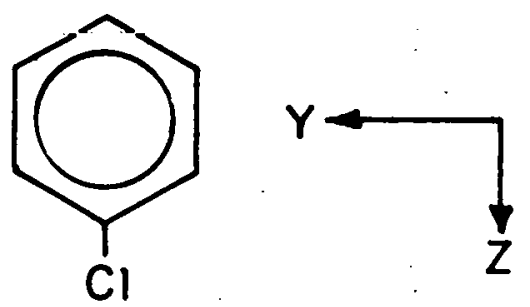

$\begin{array}{llllllllllll}X & X & X & X & Y & Y & Y & Y & Z & Z & Z & Z\end{array}$

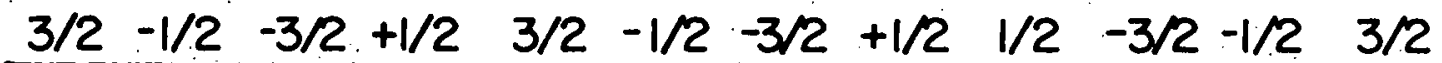

\begin{tabular}{|c|c|c|c|c|c|c|c|c|c|c|c|}
\hline \multirow[t]{2}{*}{$x+A$} & & & $\cdots$ & $\begin{array}{r}-3 / 2 \\
\text { Az2 } \\
\end{array}$ & & & & & & & \\
\hline & $x-A$ & & & & $\begin{array}{l}+1 / 2 \\
A Z Z\end{array}$ & & & & & & \\
\hline & & $X+A$ & & & & $\begin{array}{l}+3 / 2 \\
A z Z\end{array}$ & & & & & \\
\hline & & & $x-A$ & & & & $\begin{array}{r}-1 / 2 \\
A \geq 2\end{array}$ & & & & \\
\hline $\begin{array}{r}-3 / 2 \\
A Z Z\end{array}$ & & & & $Y+A$ & & $\therefore$ & & $\begin{array}{l}\sqrt{3 / 2} \\
A \times x\end{array}$ & & & \\
\hline & $\begin{array}{c}+1 / 2 \\
A Z Z\end{array}$ & & & & $Y-A$ & & & $A x x$ & $\begin{array}{l}\sqrt{3} / 2 \\
A \times x\end{array}$ & & \\
\hline & & $\begin{array}{l}+3 / 2 \\
A z Z \\
\end{array}$ & & & & $Y+A$ & & & & $\begin{array}{l}\sqrt{3} / 2 \\
A \times x \\
\end{array}$ & \\
\hline & & & $\begin{array}{r}-1 / 2 \\
A \geq 2 \\
\end{array}$ & & & & $Y-A$ & & & $A x x$ & $\begin{array}{l}\sqrt{3} / 2 \\
A \times x\end{array}$ \\
\hline & & & & $\begin{array}{l}\sqrt{3} / 2 \\
A x x\end{array}$ & $A \times x$ & & & $Z-A$ & & & \\
\hline & & & & & $\begin{array}{l}\sqrt{3} / 2 \\
\text { Axx }\end{array}$ & & & & $Z+A$ & & \\
\hline & & & & & & $\begin{array}{l}\sqrt{3} / 2 \\
\mathrm{~A} \times \mathrm{x}\end{array}$ & $A x x$ & & & $Z-A$ & \\
\hline & & & & & & & $\begin{array}{l}\sqrt{3 / 2} \\
A \times x\end{array}$ & & & & $Z+A$ \\
\hline
\end{tabular}

XBL $7012-7269$

Figure 11

Hamiltonian Matrix for the Triplet Plus one $I=3 / 2$. Nuclear Spin Considering only the $A_{x x}$ and $A_{z z}$ Hyperfine Components 


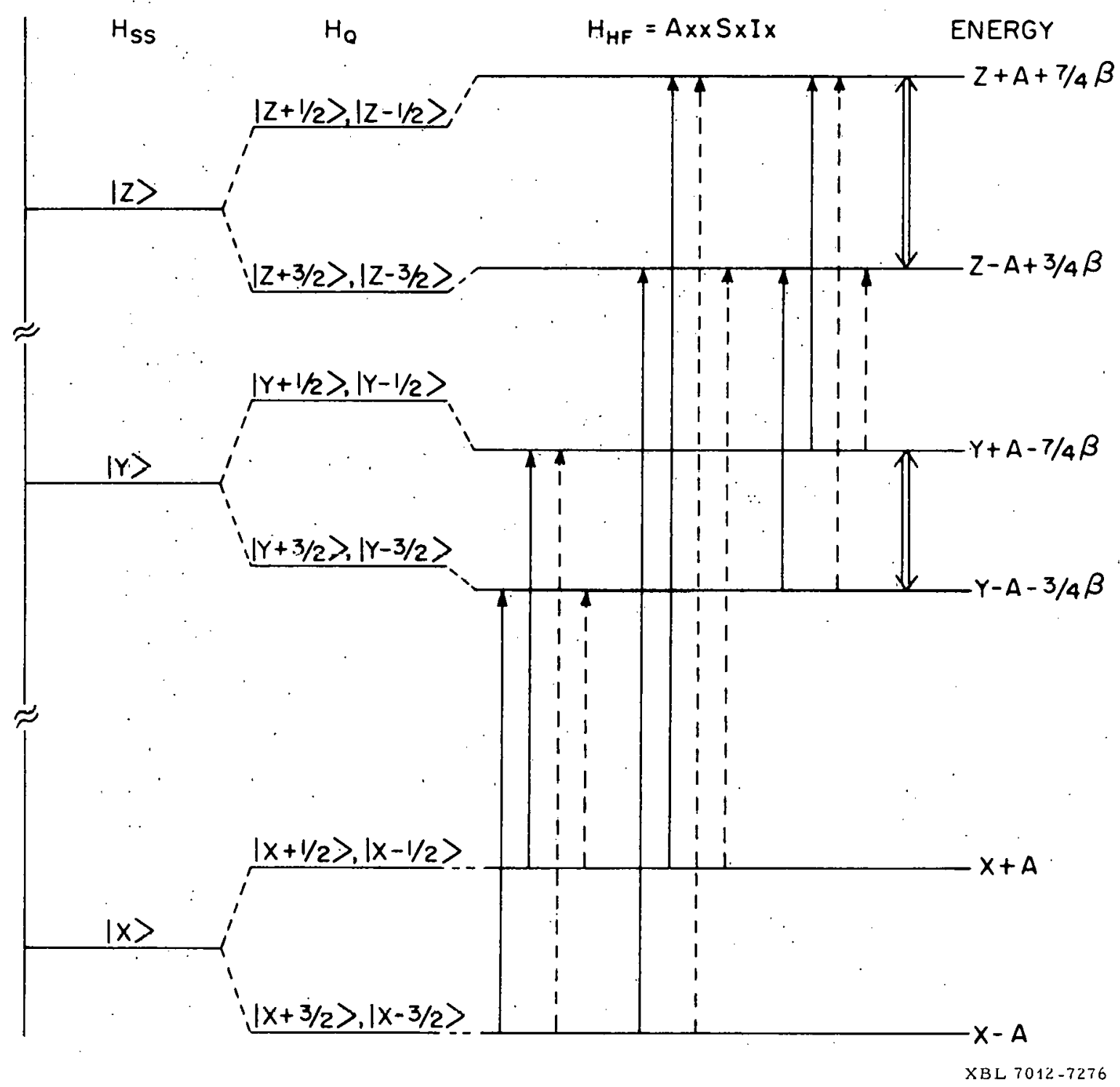

Figure 12

Energy Level Diagram for the Triplet and One $I=3 / 2$ Nuclear Spin Considering only the $A_{x x}$ Hyperfine Component 
a) $\tau_{x} \rightarrow \tau_{z}$

$\nu_{0}=z-x$

b) $\tau_{x} \rightarrow \tau_{y}$

$\nu_{0}=y-X$

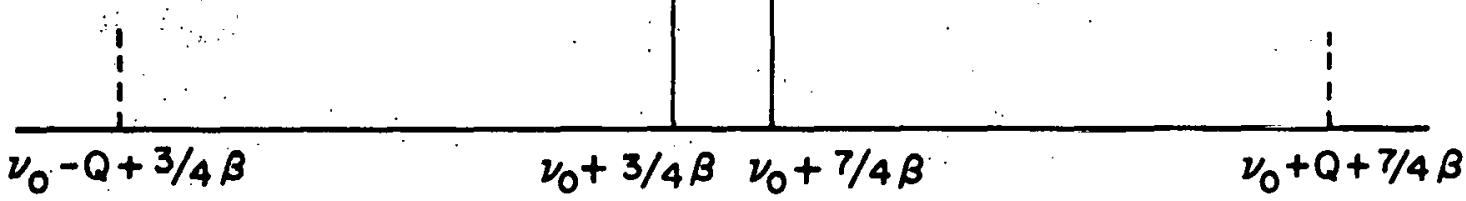

c) $\tau_{y} \rightarrow \tau_{z}$

$\nu_{0}=Z-Y$

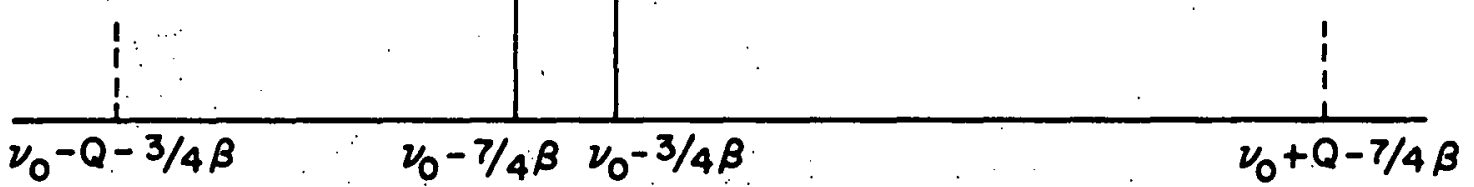

Figure 13

ODMR Spectra Predicted for the Fnergy Level Diagram Shown in Figure 12 


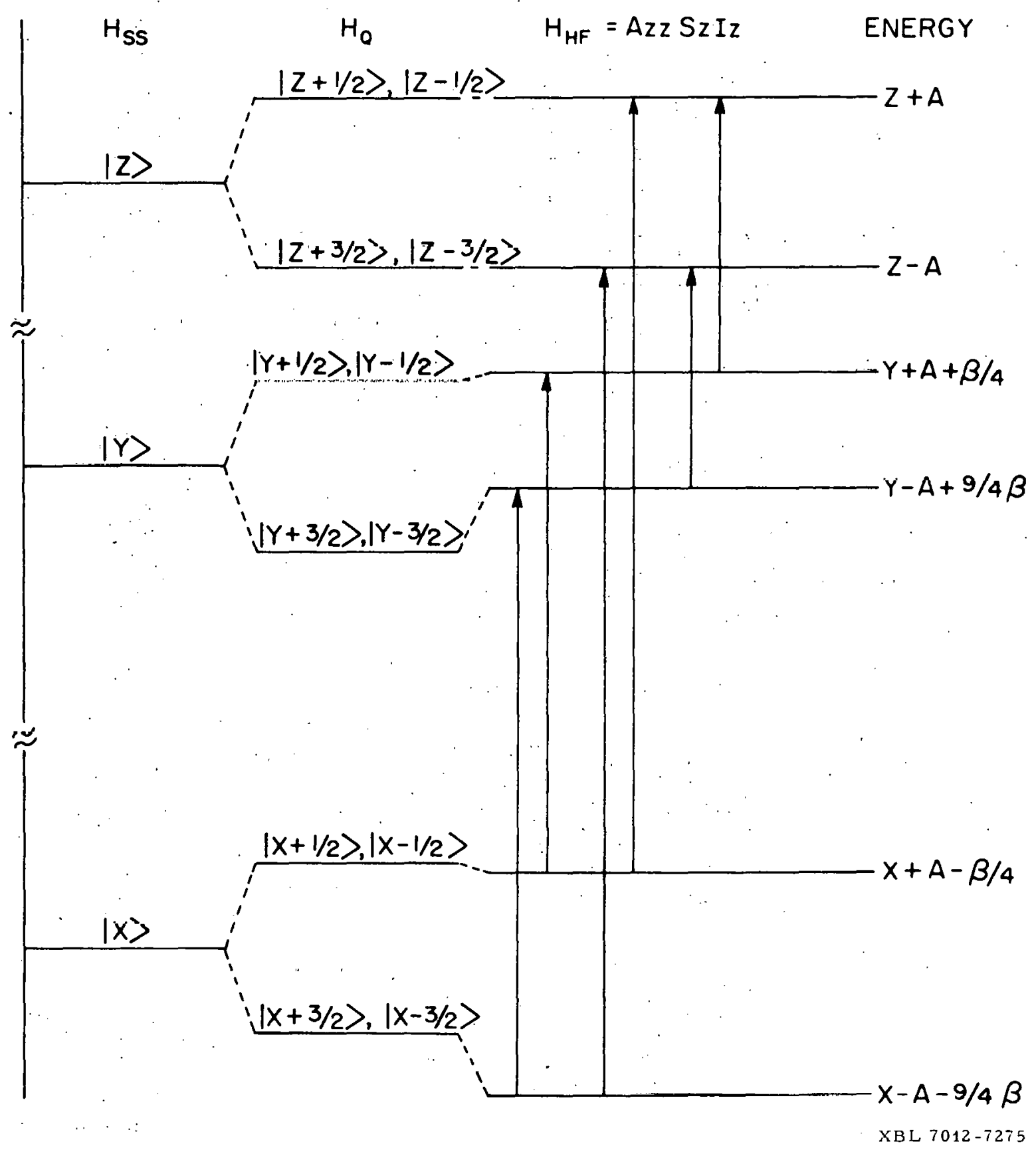

Figure 14

Energy Level Diagram for the Triplet and One $I=3 / 2$. Nuclear Spin Considering only the $\mathrm{A}_{\mathbf{z z}}$ Hyperfine Component 

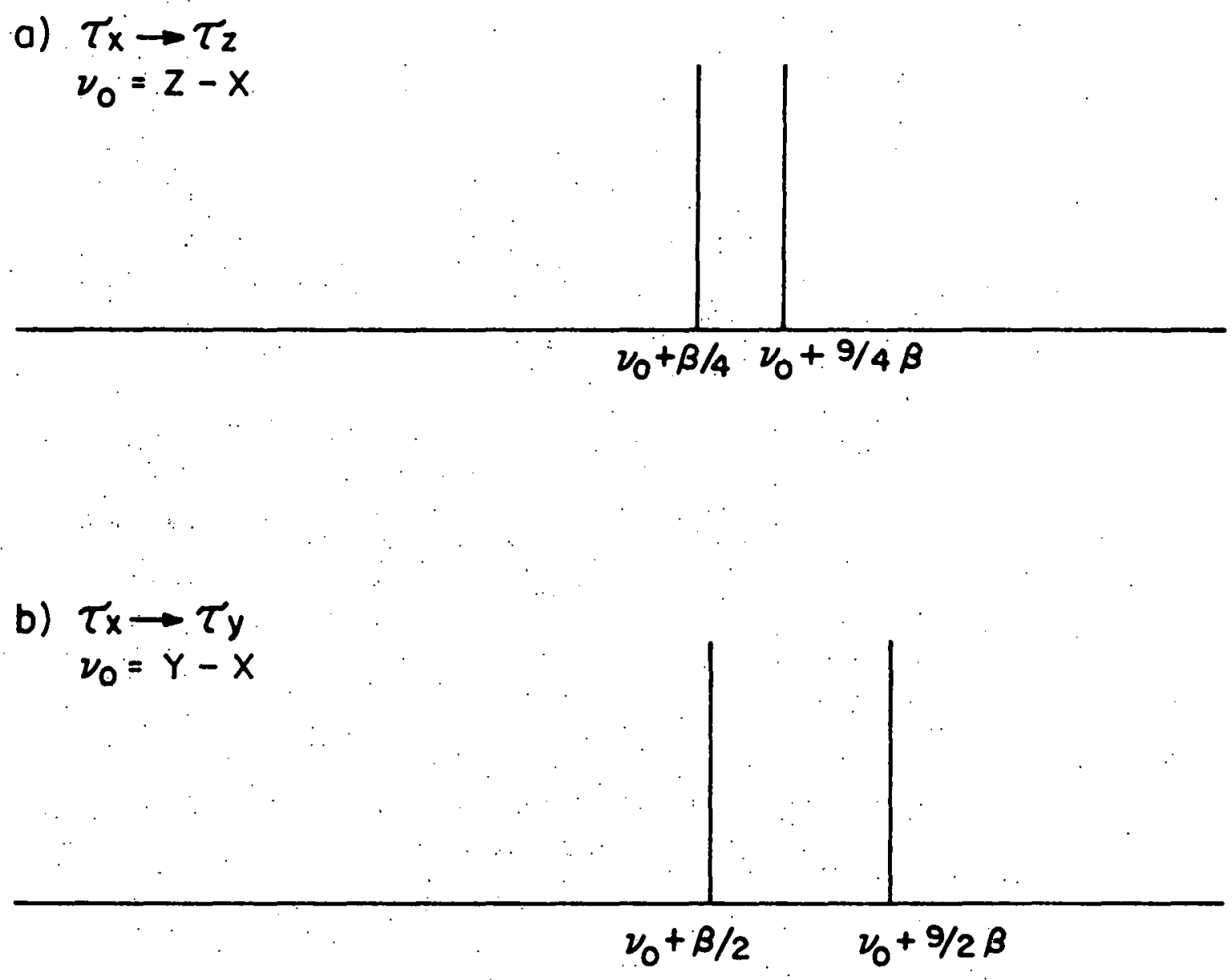

c) $\tau_{y} \rightarrow \tau_{z}$

$$
\nu_{0}=Z-Y
$$

Figure 15

ODMR Spectra Predicted for the Energy Levcl Diagram Shown in Figure 14 
The basis states that are coupled by $A_{x x}$ may be represented graphically as

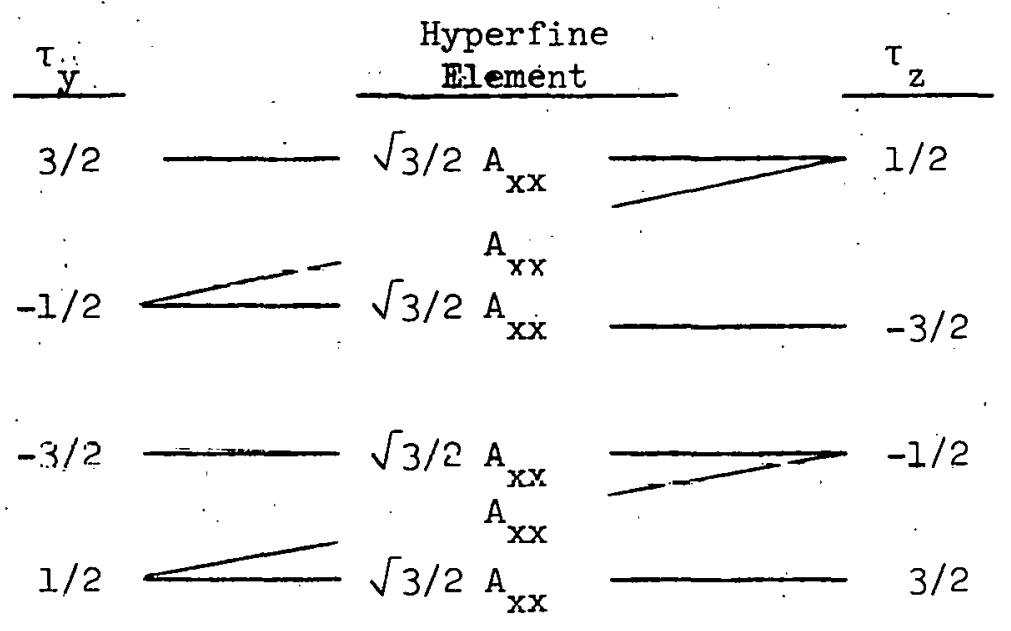

The effect of the perterbation treatment is that, to second order, the energy of the $\pm 3 / 2$ nuclear levels in the $\tau_{y}$ manifold are shifted to higher energy by an amount $3 / 4 \beta$, where $\beta$ is given by

$$
\beta=\frac{A x^{2}}{E y-E z}
$$

and the $\pm 3 / 2$ level in the $\tau_{z}$ manifold is shifted to lower energy by an equal amount.

The $\pm 1 / 2$ Jevels of the $\tau_{y}$ manifold are shifted to higher energy by an amount $7 / 4 B$ while the $11 / 2$ levels in the $\tau_{z}$ manifold are shifted an equal amount to lower energy. The resulting energy level diagram. is shown in Fig. 12. Since this spin system has a total spin that is a half integer (5/2), it is a Kramers doublet, and therefore all the energy levels are twofold degenerate in the absence of an external magnetic field. 
The hyperfine coupling therefore will never remove the degeneracy of the \pm nuclear levels and consequently there are only six energy levels to consider.

In Fig. 13 the spectra corresponding to the three ESR transitions is shown. The "allowed" structure on each transition is split into two components separated by $\beta$ for both the $\tau_{x}+\tau$ and the $\tau_{y}+\tau_{z}$ transitions and $2 \beta$ for the $\tau_{y} \rightarrow \tau_{z}$ transitions.

The "forbidden" transitions, shown by the dotted lines in Fig. 13, correspond to simultaneous electron nuclear flips. The separation of the two forbidden satellites in each ESR transition is equal to $e^{2} q Q+B$, $e^{2} q Q-B$ and $e^{2} q Q$ for the $\tau_{x} \rightarrow \tau_{z}, \tau_{x} \rightarrow \tau$ and $\tau_{y} \rightarrow \tau$ transitions respectively.

It is interesting to note that if only the perturbation due to the Ay component of the hyperfine tensor is considered, the results may be obtained by an appropriate relabeling of Figs. 12 and 13. However, if only the $A_{z z}$ component is considered, the nuclear sublevels in the $\tau_{x}$ manifold-are mixed with those in the $\tau_{y}$ manifold having the same value of the $I_{z}$ quantum number;

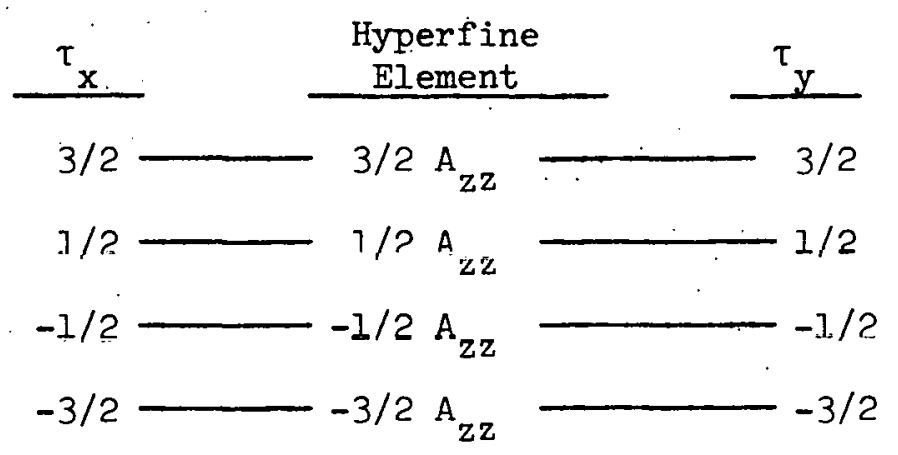

therefore, no shtel, ites due to nuclear transitions are observed. The resulting energy level diagram, considering only the $A_{z z}$ component of the hyperfine tensor is given in Fig. 14 and the resulting spectra in Fig. 15. 
The ENDOR transitions permitted by the electron dipole moment transition operator, for the case with only the $A_{x x}$ hyperfine element, are shown by the double arrows in Figure 12. The analysis of the ENDOR spectra follows the same method as that for a spin one nucleus, with the same expression for the intensity of the transitions induced by the electron magnetic dipole moment transition operator and the nuclear mak;netic dipole moment transition operator. When only the $A_{2 z}$ hyperfine element is present, the electron magnetic dipole transition operator $1:$ ineffective in producing ENDOR transitions and consequently the intensi.ty of any observed ENDOR signal is due solely to the nuclear magnetic dipole transition operator.

Some generalizations can be made at this point concerning the appearance of "f'orbldden" sateliftes whose separallun is in zero th order the pure nuclear quadmpole transition frequency of the molecule in an excited triplet state. (a) For a nuclear $\operatorname{spin} \pm=1$ (e.g. ${ }^{14} \mathrm{~N}$ ) a hyperfine element associated with a direction $i, A_{i i}$, gives intensity into a simultaneous electron-nuclear flip in the plane normal to $i$. Thus, at least two nuclear hyperfine elements must be finite to oblain independently both $e^{2} q Q$ and $\eta$. (b) For a nuclear spin $I=3 / 2$ (e.g. ${ }^{35} \mathrm{Cl}$ ), a nuclear hyperfine element parallel to the principal axis. of the field gradient (i.e., $A_{z z}$ ) does not introduce mixing between electron-nuclear states that admit intensity into forbidden satellites. (c) For a nuclear spin $I=3 / 2$, a nuclear hyperfine element perpendicular to the principal axis of the field gradient introduces intensity into forbidden sateliites whose separation in zero ${ }^{\text {th }}$. order is the pure 
nuclear quadrupole transition frequency; however, $e^{2} q Q$ and $\eta$. can never be obtained independently in the absence of an external magnetic field.

Although we have not treated explicitly the case where two nuclei are present on the same molecule, both having nuclear spin $I \geq 1$, the generalizations (a)-(c) hold with one additional feature being manifested, that is the possibility of simultaneous multiple nuclear-electron spin flips. In addition, simultaneous multiple nuclear ENDOR transitions are expected and, indeed, observed. 


\section{EXPERTMENTAL EQUIPMENT AND PROCEDURE}

- In this section the procedures and equipment used in performing both the ESR and ENDOR experiments are given. In addition, some variations of the basic experiments are listed and their advantages discussed.

\section{A. The ESR Experiment}

l'he basic experimental arrarigement is shown in Figurs lí. 'l'he sample is mounted inside a helical slow wave structure ${ }^{72}$ which is attached to a rigia stainless steel coaxial line suspended in a liquid helium dewar. The exciting light is supplied by a PEK 100-watt mercury short arc lamp mounted in a PEK M912 lamp housing. The output of the lamp is flltered by either an interference filter centered at $2500 \AA, 2800 \AA$ or $3100 \AA$; or a combination of Corning glass and solution filters. ${ }^{73}$

The phosphorescence is collected at a 90 degret angle to thc crciting light and focused through an appropriate Corning filter (to remove scattered light) and onto the entrance slit of a Jarrel-Ash model 48-490, $3 / 4$ meter spectrometer. The spectrometer has on $I$ number of 6.5 and is equipped with adjustable curved slits. The resolving power of the spectrometer is greater than 30,000 with a dispersion of' 8 . ' $2 \AA / \mathrm{mm}$ in 11 rst order. The spectral range in air is from 1900 to $16,000 \AA$.

The light at the exit slit is detected with an FMI $6256 \mathrm{~s}$ photomultiplier mounted in an EMI dry-ice cooled housing. A Fluke 4ljB power supply is used to maintain the cathode of the photomultiplier at $-1800 \mathrm{~V}$. The output of the photomultiplier is connected to a Keithly model $610 \mathrm{CR}$ 


\section{ESR EXPERIMENT}

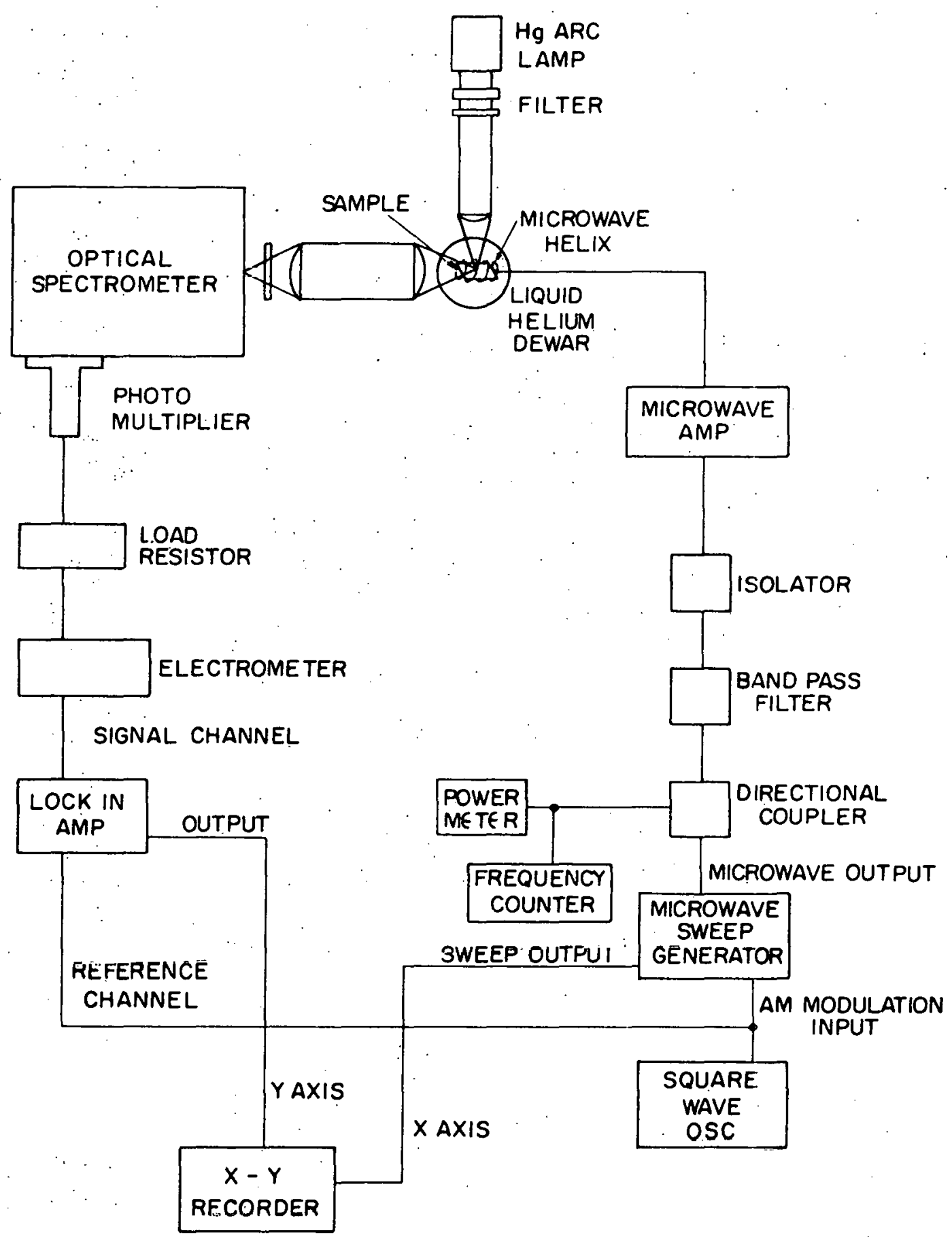

XBL $7012-7367$

Figure 16

Experimental Arrangement for Optically Detected Electron Spin Resnnanre in 7ero Magnotio Ficld 


\section{B. The ENDOR Experiment}

The experimental arrangement usually employed is shown in Figure 17 . An enlarged view of the sample, helix, and ENDOR coils is shown in Figure 18. The optical and microwave equipment is the same as that used in the ESR experiment with the exception that the microwave field $\left(\mathrm{H}_{l}\right)$ is not modulated: The radiofrequency field $\left(\mathrm{H}_{2}\right)$ is supplied by a Hewlett Packard model $8601 \mathrm{~A}$ sweep osciliator that covers the region from 0.1 to $110 \mathrm{MHz}$. The output is modulated by an E G \& G model LGiOl/N Inear yate. The gatc is driven by the square wave generator which also drives the reference channel of the lock-in amplifier.

The RF is then amplified by two broad-band distributed amplifiers, ${ }^{74}$ a four watt unit (see Figure 19) and a 20 watt unit (Figure 20), and connected to the ENDO $\bar{R}$ coils. I'hese amplifiers lave the advantagc that thay operate over the range of 1 to $50 \mathrm{MHz}$ without the need of adjustment. A series of thcoc amplifiers was designed and constructed in collaboration with Bob Smith and Bill Gagnon of Lawrence Radiation Laboratory for use in pulsed nuclear quadrupole resonance experiments. Since these amplifiers are a significant advancement in high power broad-band umplifiers, the schematics are shown in Figures 21-26. The largest unit will produce 20,000 watt. pulses of RF with a 20 percent duty cycle.

The ENDOR coil consists of a "bridge $T$ " constant resistance network 75 in a Helmholtz arrangement as shown in Figure 18.

The $x$ axis of the recorder is driven by the ramp voltage from the RF sweep oscillator and the $y$ axis from the output of the lock-in amplifier. 


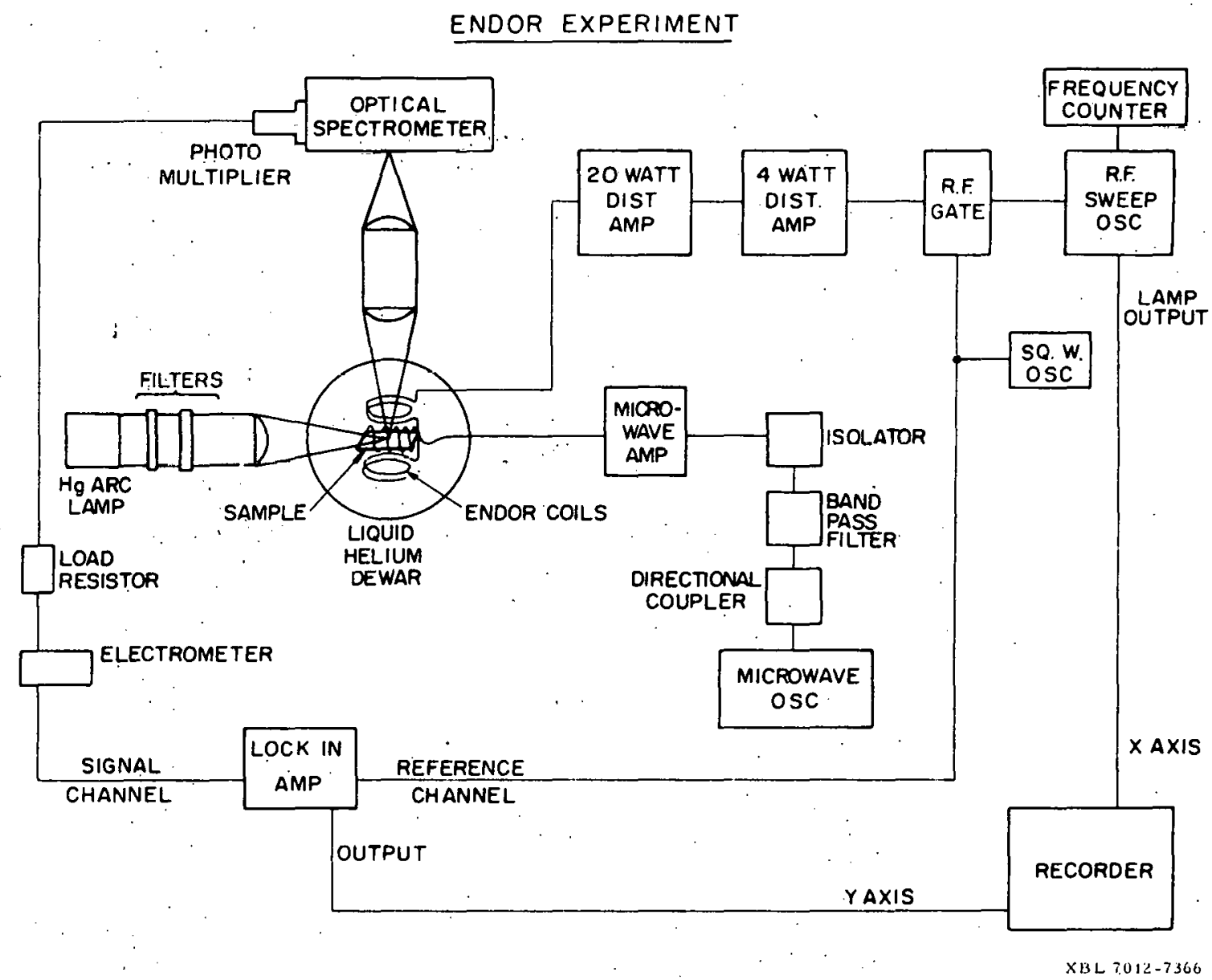

Figure 17

Experimental Arrangement for Optically Detected ENDOR in Zero Magnetic Field 


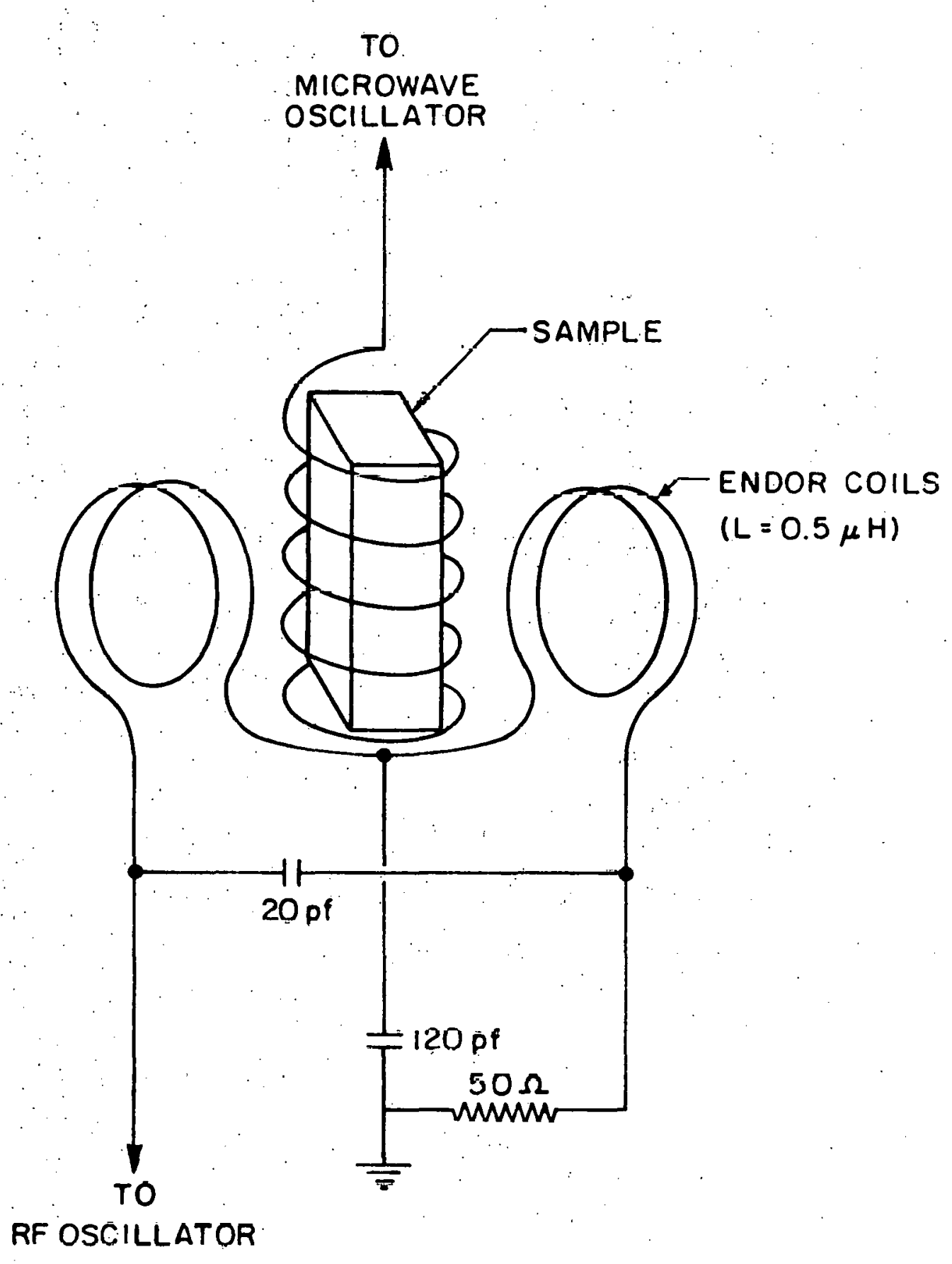

Figure 18

Sample Arrangement and ENDOR Coil Schematic 


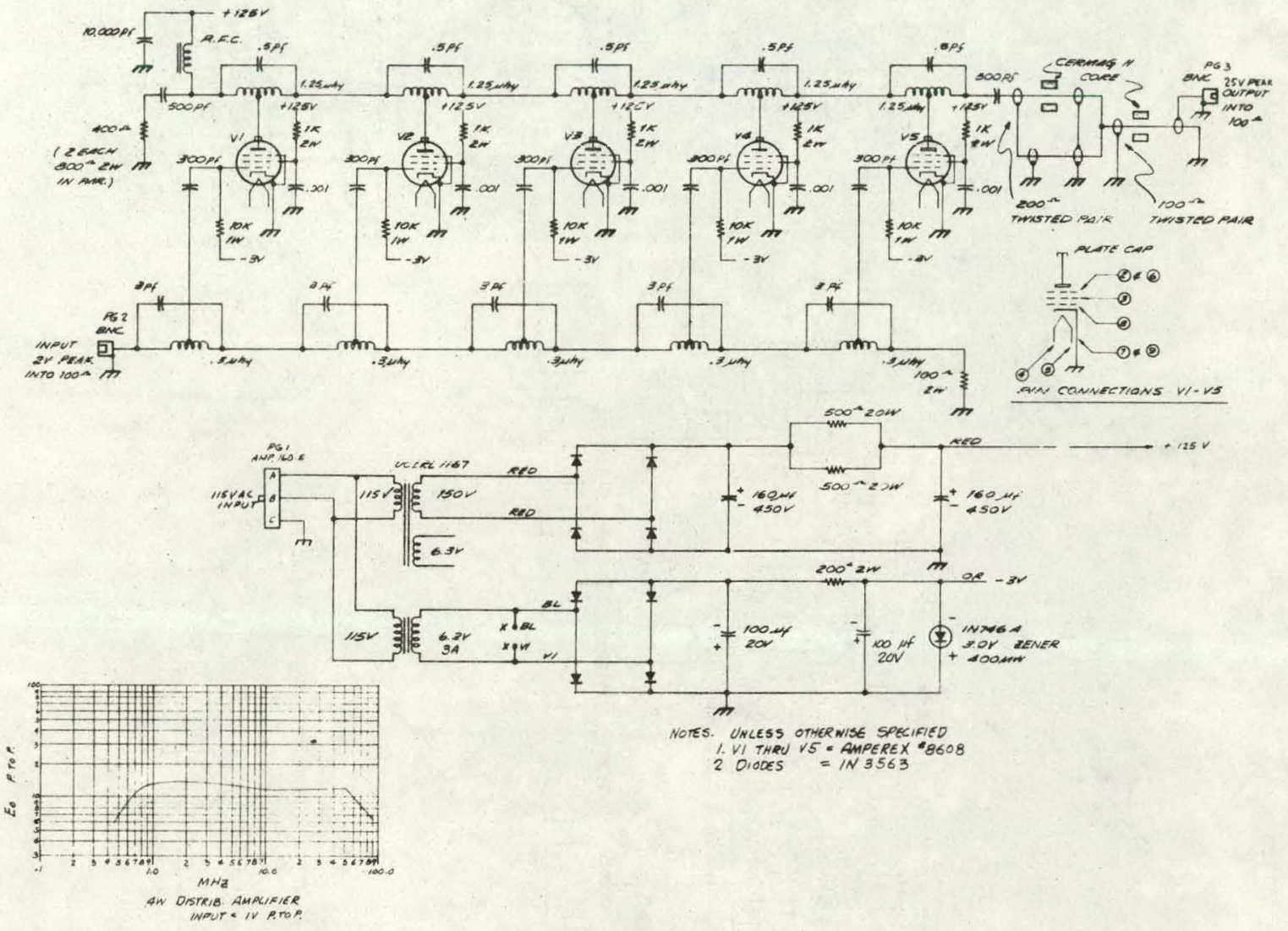

XBL $7012-7278$

Figure 19

1 Watt Distributive Amplifier Schematic 


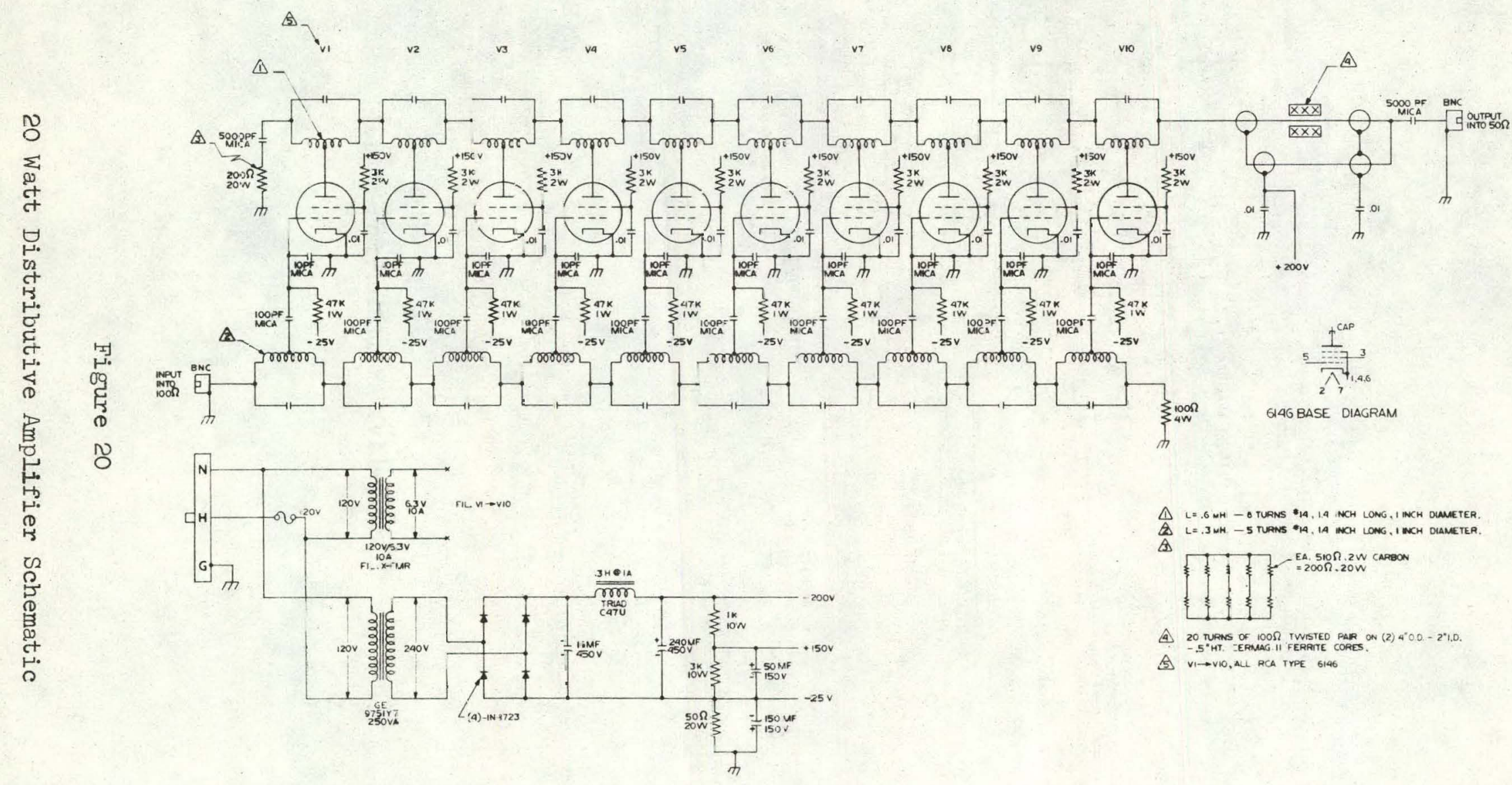




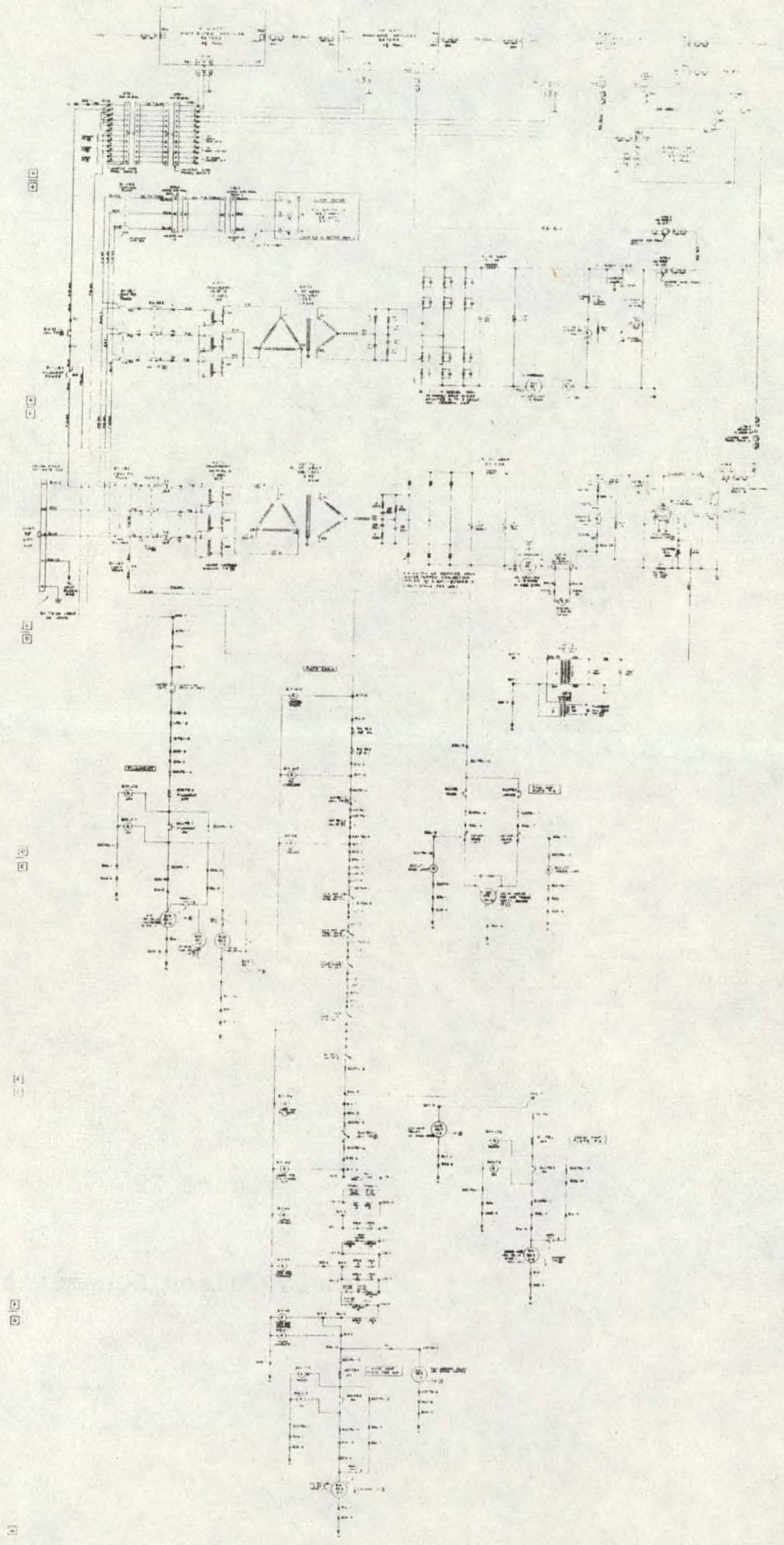

XBL $7012-7353$

Figure 21

Broad Band Distributive Amplifier System Master Schematic 


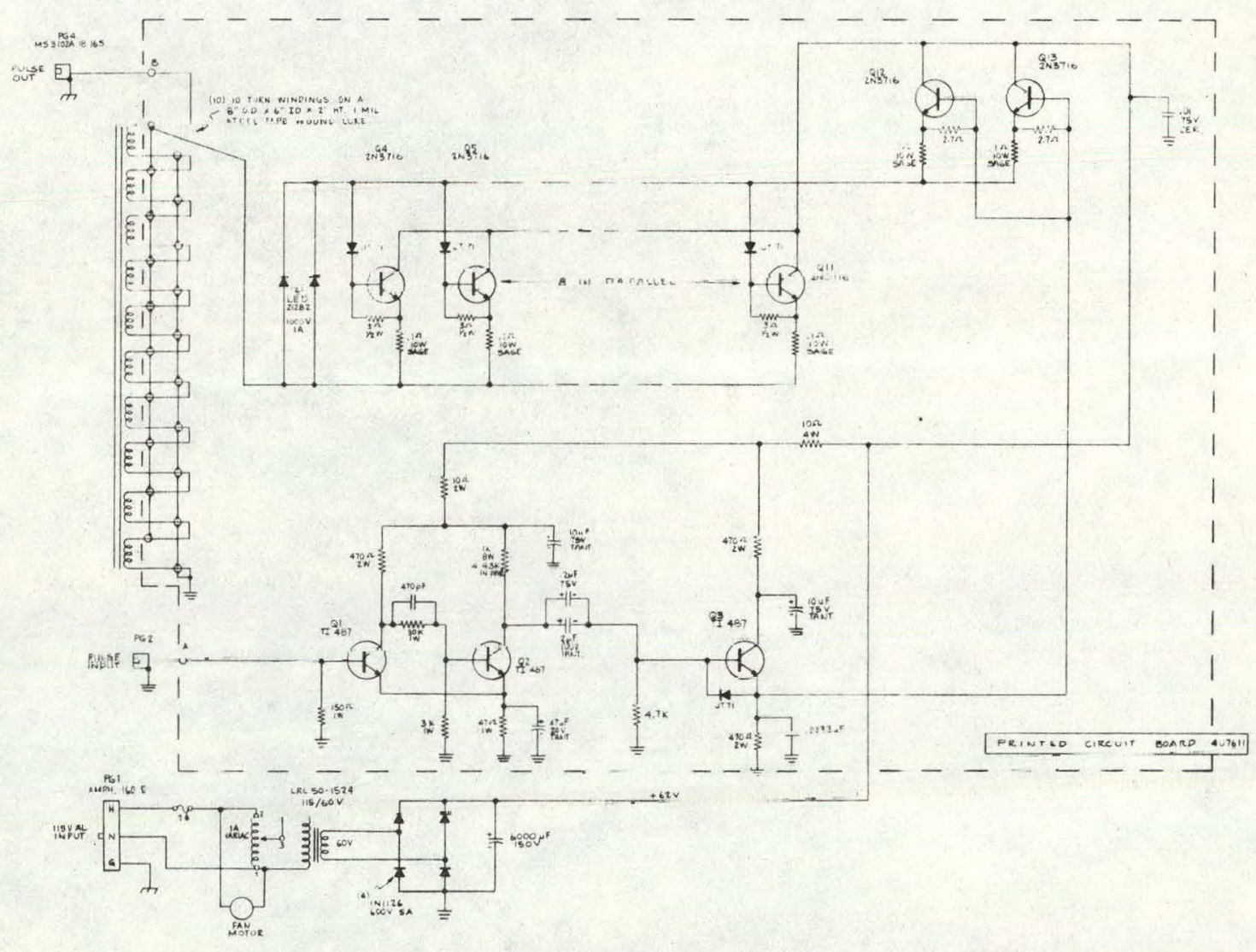

XBL $7012-7351$

Figure 22

Grid Pulser Schematic 


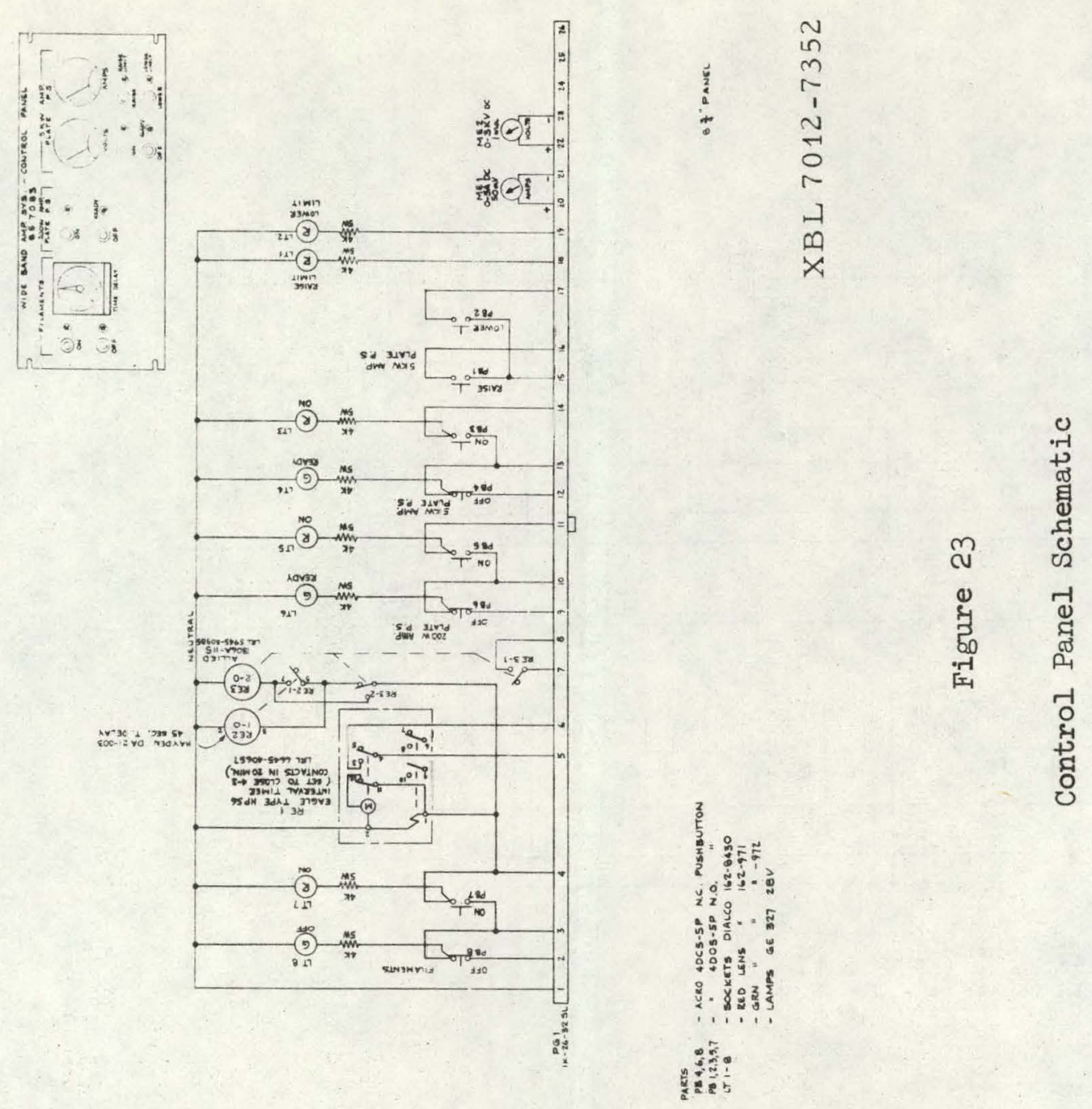




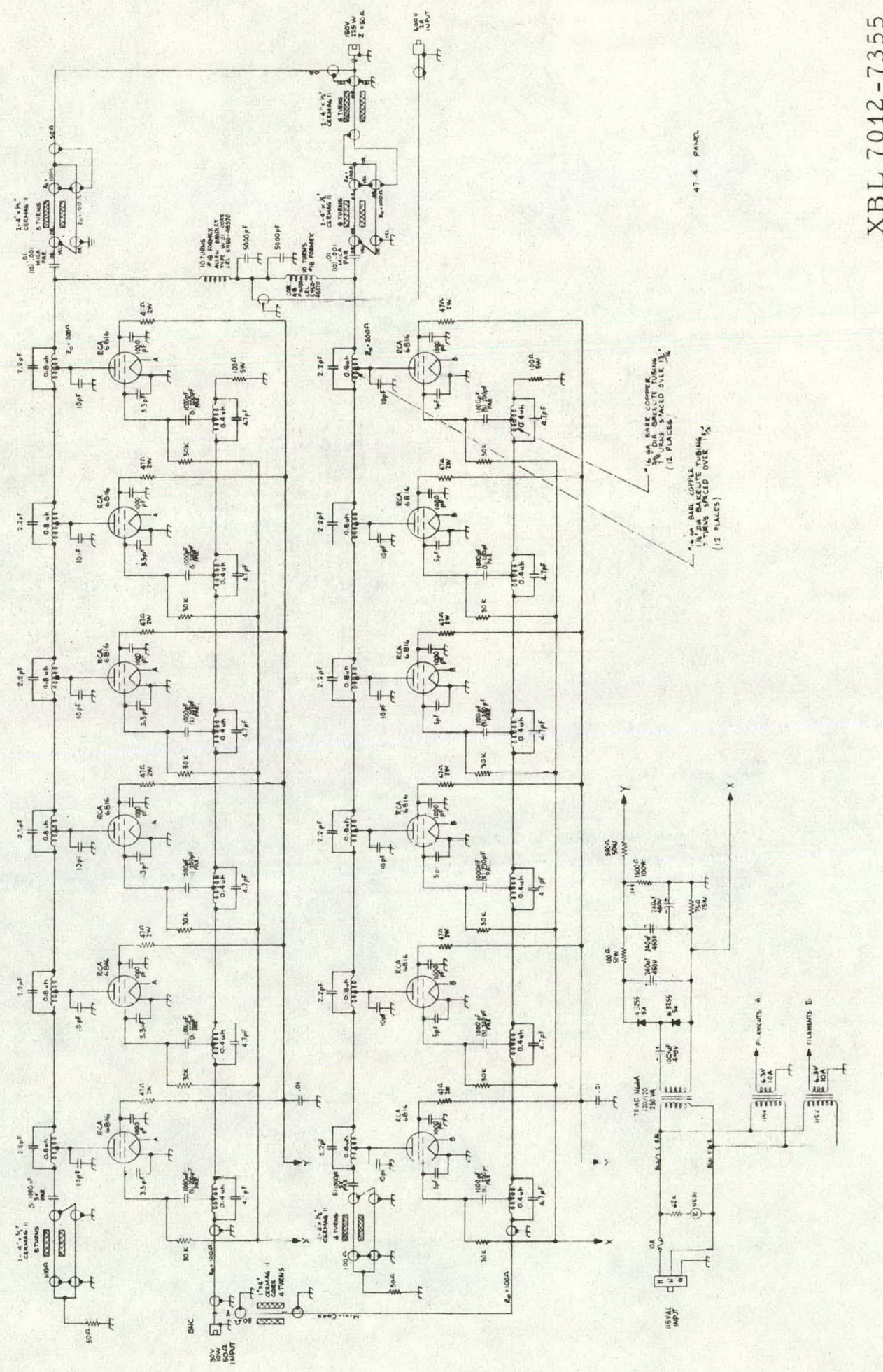

Figure 24 


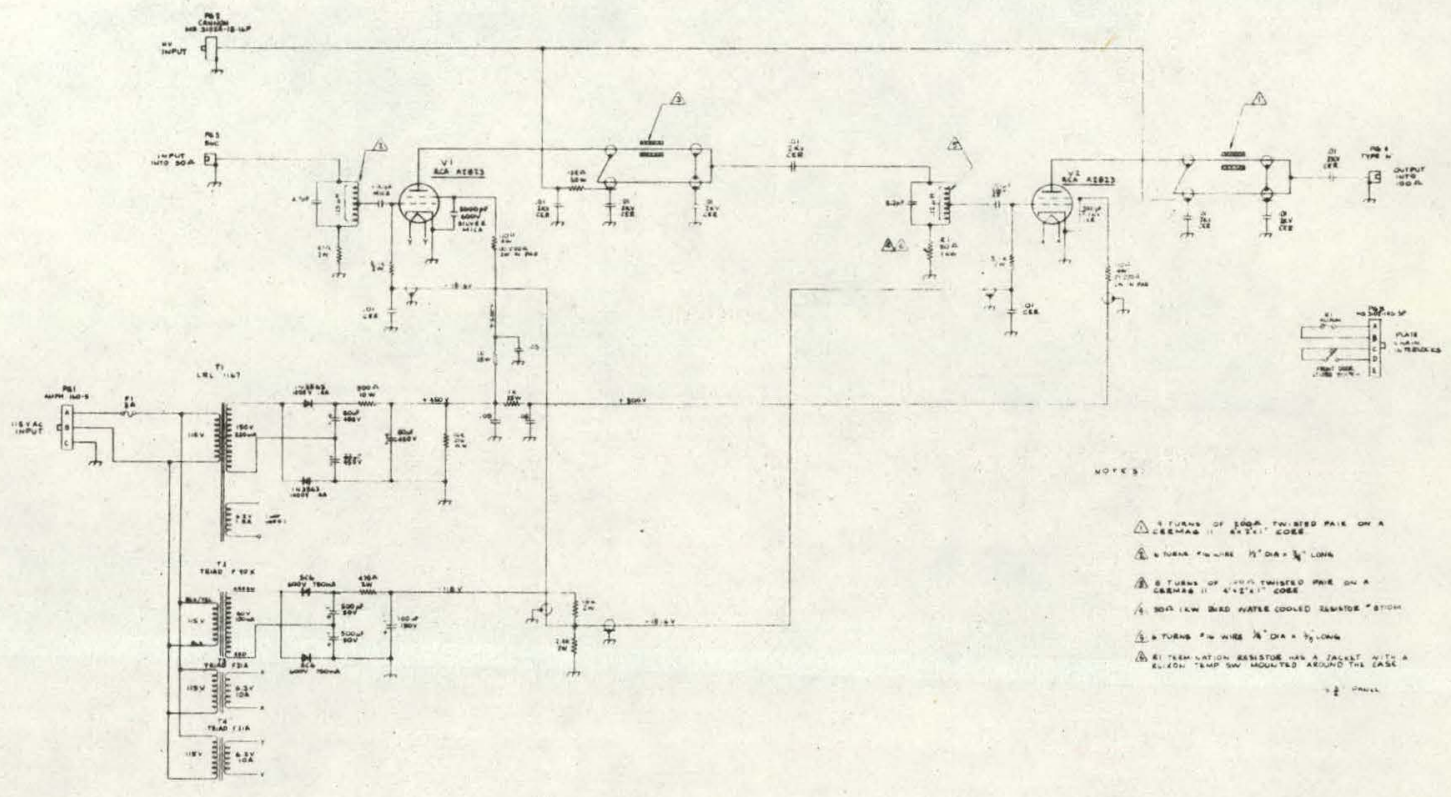

XBL $7012-7277$

Figure 25,

200 Watt Distributive Amplifier Schematic 


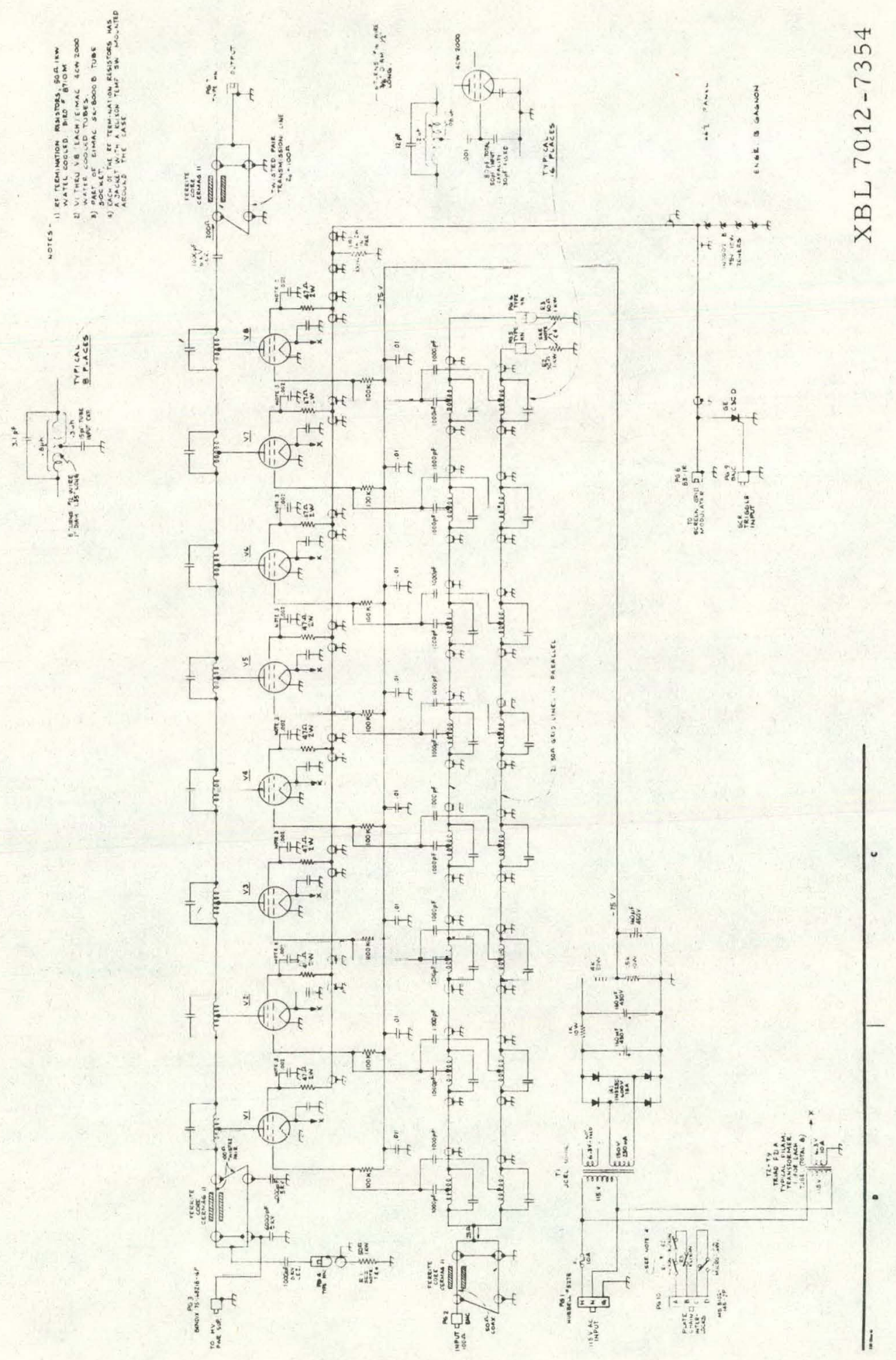

Figure 26

10,000 Watt Distributive Amplifier Schematic 


\section{Variations of the Basic Experiments}

The optical detection of magnetic resonance permits several additional parameters to be experimentally adjusted. These include the energy and bandwidth of the phosphorescence that is monitored as well as the energy, bandwidth and intensity of the exciting light. In addition, the power of the microwave field $\mathrm{H}_{1}$ may be adjusted over a wider range than in experiments in which the absorption of microwave power is monitored. This is due to the fact that saturation gives the maximum signal strength using optical detection techniques, while with absorption experiments the signal strength will decrease as the power is increased above that needed for saturation. The advantage of this is that the signal strength of weak "forbidden". transitions may be improved by the application of large $\mathrm{H}_{1}$ fields without a decrease in the signal strength of the allowed transitions.

Some of the most useful variations of the basic experiment are listed in Table IV. If a high resolution spectrometer is employed to isolate the phosphorescence emission, the optically detected ESR may be used to simplify the phosphorescence spectrum by amplitude modulation of the $\mathrm{H}_{1}$ field while saturating an ESR transition. The modulation of the phosphorescence is detected with a phase sensitive amplifier while sweeping the optical spectrum. Since only two of the three triplet levels are coupled by the $H_{1}$ field, only the emission from these two levels will be detected. Therefore, by repeating the experiment while saturating the remaining two ESR transitions, three spectra are obtained, each including only the emission from two of the three zero field levels. 
electrometer through an adjustable load resistor. The output of the electrometer is connected to the signal channel input of a PAR model HR-8 lockin amplifier.

The microwave field is supplied by a Hewlett Packard sweep oscillator model $8690 B$. equipped with plug-in units to cover the range from $\cap .1$ to 18 GHz. The output is connester tin a Servo serp. traveling wave tubs nmplifier ( 1 to $12 \mathrm{GHz}$ at 1 watt, \#3003, 1 to $2 \mathrm{GHz}$ at 20 watts, \#2210; and 2 to $4 \mathrm{GHz}$ at 20 watts, \#2220). The output of the amplifier is then fed consecutively through a directional coupler, a band-pass filter, and an isolator to the rigid coaxial line on which the helix is mounted.

The microwave sweep oscillator is amplitude modulated with a HewlettPackard model 21 IR square wave generator which is also connected to the reference channel of the lock-in amplifier. The output of the lock-in amplifier drives the $\mathrm{y}$ axis of a Hewlett Packard model $004 \mathrm{~B}$ recorder while the ramp voltage from the microwave sweep oscillator drives the $x$ axis.

The temperature of the sample is usually lowered to approximately $1.3^{\circ} \mathrm{K}$ by pumping on the liquid helium with three Kinney model KTC-2l vacuum pumps operated in parallel.

The ESR experiment is performcd by monitoring the change in twissiun of the sample while varying the frequency of the modulated microwave field. As explained in section II, the signal may either increase or decrease. With a lock-in amplifier a decrease in emission intensity corresponds to a phase shift of 180 degrees relative to the signal obtained for an increase in emission intensity. 
The information obtained from the analysis of phosphorescence spectra is extremely useful by itself in characterizing the triplet state, and complementary to the information obtained from the analysis of the ESR spectrum.

In ENDOR experiments the radiofrequency field $\mathrm{H}_{2}$ may also be adjusted. These experiments are usually performed by saturating an ESR transition while varying the frequency of the $\mathrm{H}_{2}$ field. Either the $\mathrm{H}_{1}$ or $\mathrm{H}_{2}$ fields may be modulated; however, it is usually preferable to modulate the $\mathrm{H}_{2}$ field since, in this case, only the change in intensity of the phosphorescence due to the ENDOR resonance is detected with a lock-in amplifier. On the other hand, if the $\mathrm{H}_{1}$ field is modulated, there is a constant signal due to the ESR transition which changes in intensity when the $\mathrm{H}_{2}$ field is swept through resonance. A useful modification of this technique is achieved by modulation of the $\mathrm{H}_{2}$ field while simultaneously saturating an ENDOR transition and sweeping the $\mathrm{H}_{1}$ field. In this case, only the ESR transitions that connect energy levels simultaneously coupled by the $\mathrm{H}_{I}$ and the $\mathrm{H}_{2}$ fields are detected. This method is useful in analyzing the structure of the ESR transition since the contribution to the spectrum due to different isotopes and/or nuclei may be isolated.

If both an ESR and an ENDOR transition are saturated while modulating the $\mathrm{H}_{2}$ field and scanning the phosphorescence spectrum, it is possible to isolate the contribution to the phosphorescence spectrum from molecules containing different nuclear isotopes. As an example, if the phosphorescence from a molecule such as chlorobenzene is monitored and a $\mathrm{Cl}^{35}$ ENDOR transition saturated while modulating the $\mathrm{H}_{2}$ field, only the 
contribution to the phosphorescence spectrum from molecules containing the $\mathrm{Cl}^{35}$ isotope will be detected. The same experiment may then be re: peated detecting only the contribution from the molecules containing the $\mathrm{Cl}^{37}$ isotope. The difficulty in this experiment is that only small, if any, variations in the phosphorescence spectrum would be expected and con= sequently, the maximum resolution of the optical spectrometer must be employed. This severely limits the signal to noise ratio of the ENDOR signal. 
TABLE IV

Techniques of Optical Detection of ESR

Excitation

Light

1) C.W.

2) C.W.

3) Chopped

4) C.W.

5) C.W.

6) C.W.

7). Sweep
Optical Spectrometer

No

Yes

Optional

Optional

Optional

Sweep

Yes
Microwave

Modulation

No

No

No

A.M.

F.M.

A.M.

A.M.

\section{Advantages}

measure absolute change in total emission

Measure absolute change in emission of particular vibronic bands

Improvement in $\mathrm{S} / \mathrm{N}$ over Methods 1 and 2 by narrow band phase sensitive detection of the phosphorescence

detect only the change in emission from either the total emission or a particular vibronic band

detect the derivative of the spectrum, helpful in resolving spectra

detect only the emission from 2 of the 3 sublevels while sweeping the optical spectrum

useful in studying the pathways of intersystem crossing 


\section{RESULTS AND DISCUSSION}

\section{A. The $\pi^{*}$ state of 8 -Chloroquinoline}

One of the primary objectives of our research is the measurement of nuclear quadrupole coupling constants of nuclei in molecules in their excited triplet state. In particular we are interested in measuring the nuclear quadrupole coupling constants of chlorine since this is probably the most thoroughly investigated of a.t. nuclei for moleculeo in their ground state. The zero field detection of the electron spin resonance a dul chlorlne ElLUR of 8-chloroquinoline in its $\pi \pi^{*}$ state has been observed and the spectrum interpreted in terms of a spin Hamiltonian which includes the ${ }^{14} \mathrm{~N},{ }^{35} \mathrm{Cl}$, and ${ }^{37} \mathrm{Cl}$ hyperfine and nuclear quadrupole interactions. In addition, chlorine satellites have been observed in the ESR and chlorine ENDOR observed for the $\pi^{*}$ state of 2-3 dichloroquinoxaline.

The sample consisted of a einglc cryotal of dureme dujed wilh approximately 0.1 mole percent 8-chloroquinoline grown from the melt. The sample was mounted inside a helical slow-wave structure with a $2 \mathrm{~mm}$ inside diameter. The helix is matched to a rigid coaxial line which is supported vertically inside a liquid helium dewar which was maintained at $1.7^{\circ} \mathrm{K}$ by pumping on the liquid helium. The $3100 \AA$ region of the mercury short arc lamp was used as the exciting source, the sample being irradiated with light through the open helix windings. The $0-0$ band of the phosphorescence of 8-chloroquinoline was detected at a $90^{\circ}$ angle to the exciting source and isolated with the Jarrell-Ash optical spectrometer. All ESR experiments were performed by using continuous 
microwave power. The resonance was detected by monitoring the change in intensity of the phosphorescence as a function of microwave power. The apparatus used for both the ESR and ENDOR experiments is essentially the same as that shown in Figures 16 and 17 except that the output of the electrometer was plotted directly since no modulation was used. The ENDOR experiments used the arrangement shown in Figure 18 which allowed both the nitrogen and chlorine resonances to be observed without having to change coils.

Two of the three electron transitions, those associated with the $\tau_{y} \rightarrow \tau_{z}$ and $\tau_{x} \rightarrow \tau_{z}$ electron spin manifolds, were observed with satisfactory resolution. The $\tau_{y} \rightarrow \tau_{z}$ multiplet is centered at 860 MHz while the $\tau_{x} \rightarrow \tau_{z}$ multiplet is centered at $3483 \mathrm{MHz}$. In both cases, under steady state exciting light conditions, the $0-0$ band phosphorescence emission increased when the microwave field coupled the respective spin manifolds. At low microwave power (10 $\mu$ watts) the strong "allowed" electron transitions were found. As the microwave power was increased (100 $\mu$ watts), "forbidden". satellites split off the major transitions by $\sim 3 \mathrm{MHz}$ were observed. At higher powers (100-1000 $\mu$ watts) "forbidden" satellites saturated and an additional multiplet of satellites were observed split $\sim 25-40 \mathrm{MHz}$ from the center section. Figure 27. illus trates a microwave power study of the $\tau_{x} \rightarrow \tau_{z}$ transition. The same basic pattern was observed for the $\tau_{\mathrm{y}} \rightarrow \tau_{\mathrm{z}}$ transitions. ${ }^{35} \mathrm{Cl}$ and ${ }^{37} \mathrm{Cl}$ ENDOR were observed by monitoring the change in the phosphorescence intensity of the $0-0$ band while saturating one of the $\tau_{y} \rightarrow \tau_{z}$ microwave transitions in the outer satellite multiplet and simultaneously sweeping 


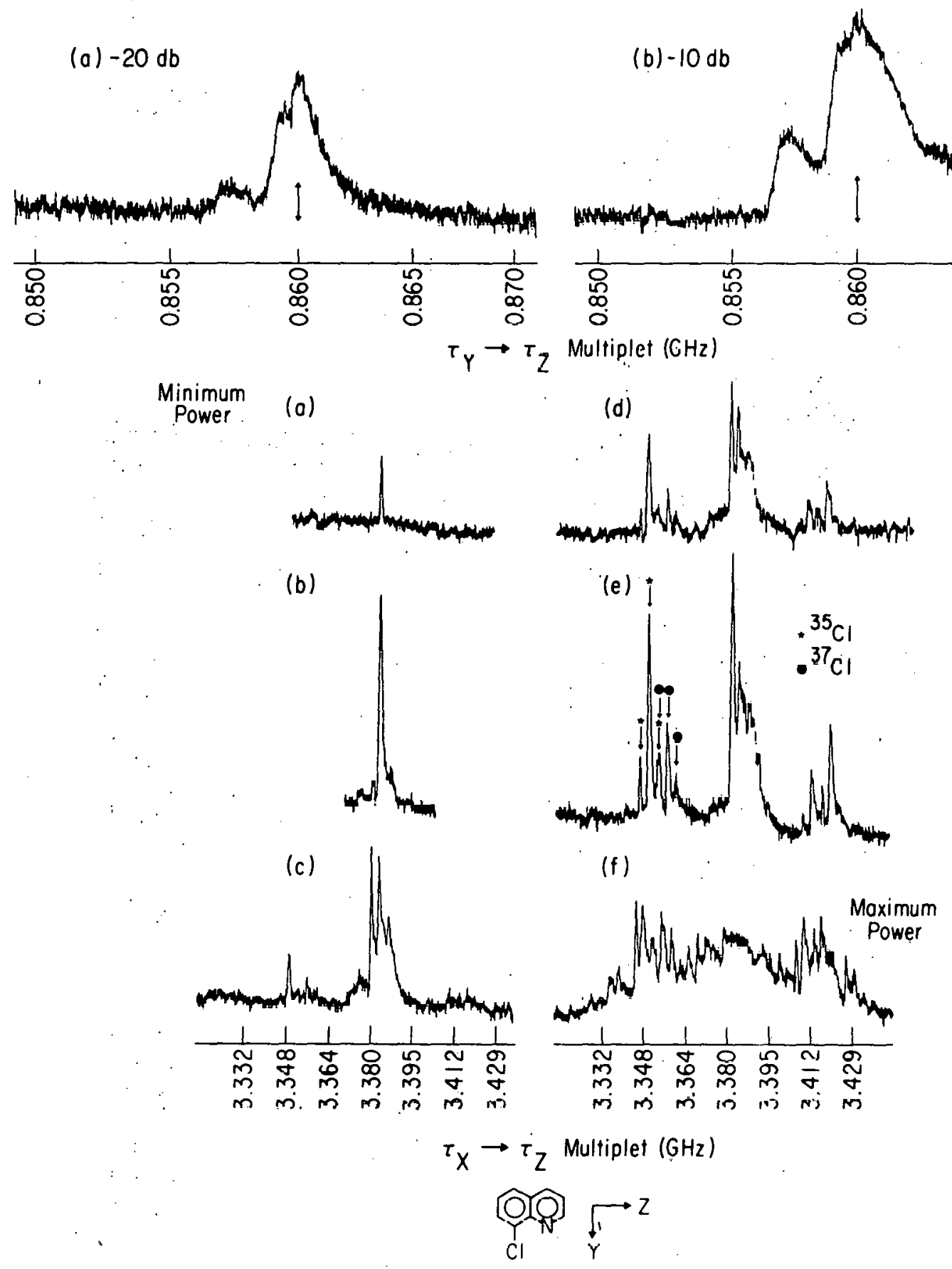

$X B L 7012-7258$

\section{Figure 27}

ODMR Transitions Observed in 8-Chloroquinoline 
the rf frequency. An example of an optically detected ${ }^{35} \mathrm{Cl}$ ENDOR in zero field is shown in Figure 28. All observed frequencies and relative intensities are given in Tables 5, 8, and 9. Measurement of the transition frequencies with an accuracy better than $\sim 0.4 \mathrm{MHz}$ was generally not possible due to the line widths associated with the individual lines ( $1.0 \mathrm{MHz}$ ) and their overlap with other transitions.

The structure of the zero field transition $\tau_{y} \rightarrow \tau_{z}$ and $\tau_{x} \rightarrow \tau_{z}$ and the accompanying ENDOR observed in 8-chloroquinoline can be understood in terms of the spin Hamiltonian already developed,

$$
\mathrm{H}=\mathrm{H}_{\mathrm{SS}}+\mathrm{H}_{\mathrm{Q}}^{\mathrm{N}}+\mathrm{H}_{\mathrm{Q}}^{\mathrm{Cl}}+\mathrm{H}_{\mathrm{HF}}^{\mathrm{N}}+\mathrm{H}_{\mathrm{HF}}^{\mathrm{Cl}}
$$

where $\mathrm{H}_{\mathrm{SS}}$ is the zero field splitting Hamiltonian

$$
\mathrm{H}_{\mathrm{SS}}=-\left(\mathrm{XS}_{\mathrm{x}}^{2}+\mathrm{YS}_{\mathrm{y}}{ }^{2}+\mathrm{ZS}_{\mathrm{z}}^{2}\right)
$$

and $\mathrm{H}_{Q}^{N}, \mathrm{H}_{Q}^{\mathrm{Cl}}$, and $\mathrm{H}_{\mathrm{HF}}^{\mathrm{N}}, \mathrm{H}_{\mathrm{HF}}^{\mathrm{Cl}}$ are the nuclear quadmupole interaction and electron-nuclear hyperfine interaction for nitrogen and chlorine. Equation 1 may be simplified by the following assumptions.

First, we assume that the $y$ and $z$ components of $\mathrm{H}_{\mathrm{HF}}^{\mathrm{N}}$ and $\mathrm{H}_{\mathrm{HF}}^{\mathrm{Cl}}$ may be neglected. This is justified insofar as the EPR measurements of Maki et al. 42,43 on quinoline have shown that $A_{x x}>A_{y y}, A_{z z}$ for the ${ }^{14} \mathrm{~N}$ hyperfine interaction. Moreover, since the perturbations due to reasonable in-plane components for either ${ }^{14} \mathrm{~N}$ or ${ }^{35,37} \mathrm{Cl}$ would be much smaller than those due to $A_{x x}$, they can, to a first approximation, be ignored. We shall also assume that the principal axes of the tensor 


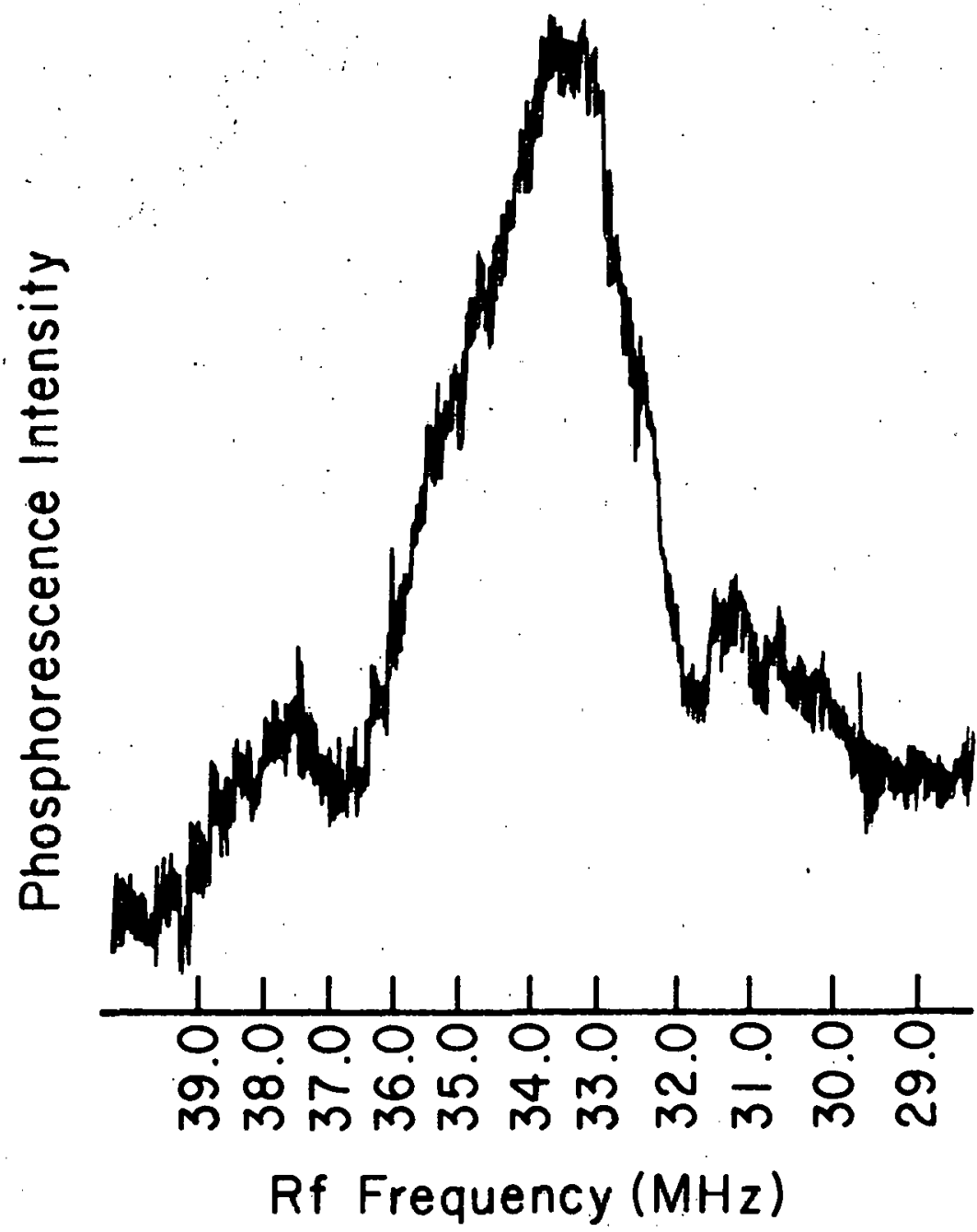

XBL $7.012-7272$

Figue 28

Optically Detected ${ }^{35} \mathrm{Cl}$ ENDOR Observed While Saturating the Microwave Transition at $826.2 \mathrm{MHz}$ in the $\tau_{\mathrm{y}} \rightarrow \tau_{z}$ Multiplet 
interactions. $\mathrm{H}_{\mathrm{SS}}, \mathrm{H}_{\mathrm{Q}}^{\mathrm{N}}$, and $\mathrm{H}_{\mathrm{Q}}^{\mathrm{Cl}}$, coincide. It is reasonable to assume that the principal axis of $\mathrm{H}_{\mathrm{Q}}^{\mathrm{N}}$ and $\mathrm{H}_{\mathrm{Q}}^{\mathrm{Cl}}$ would be close to the direction of the nitrogen lone pair orbital and along the $\mathrm{C}-\mathrm{Cl}$ bond respectively (approximately along the $Y$ molecular axis). Furthermore, in quinoline the nitrogen lone pair direction and the $Y$ axis of $H_{S S}$ are within a few degrees of each other. ${ }^{42,43}$ we assume that 8-chloroquinoline does not deviate appreciably in this respect. With these approximations we can write*

$$
\begin{aligned}
& H_{Q}^{N}=-x_{x}^{2}-y I_{y}^{2}-2 I_{z}^{2} \\
& \mathrm{H}_{Q}^{\mathrm{Cl}}=\mathrm{H}_{\mathrm{Q}}^{\mathrm{Cl}}=\mathrm{e}^{2} \mathrm{qQ}(\mathrm{Cl})\left[3 \mathrm{I}_{\mathrm{z}}{ }^{2}-15 / 4\right] / 12+\mathrm{H}_{\mathrm{Cl}}^{\mathrm{Cl}}(\eta) . \\
& \mathrm{H}_{\mathrm{Q}}^{\mathrm{Cl}}(\eta)=\mathrm{e}^{2} \mathrm{qQ}(\mathrm{Cl}) \eta\left[\mathrm{I}_{+}{ }^{2}+\mathrm{I}_{-}^{2}\right] / 24 \\
& \dot{H}_{H F}^{N}=\cdot A_{x x}(N) I_{x} S_{x} \\
& H_{H F}^{C l}=A_{X X}(C l)(I / 2 i)\left[I_{+} S_{X}-I_{-S}\right]
\end{aligned}
$$

With this coordinate system the nitrogen and molecular axis are coincident, the chlorine $x$ axis is along the molecular $x$ axis, the chlorine $y$ axis along the molecular $z$ axis and the chlorine $z$

* We have defined $I_{x}$ as $(I / 2 i)\left[I_{+}-I_{-}\right]$rather than the conventional (I/2) $\left[I_{+}+I_{-}\right]$: In our definition the Hamiltonian matrix is thus real so long as $A_{y z}(C l)$ and $A_{z y}(C l)=0$. We could have reversed the molecular $x$ and $y$ axes and used the standard convention but we chose to have the axis system for quinoline 25,26 and 8 -chloroquinoline the same. 
axis is along the molecular $y$ axis. Therefore we will designate the out of plane chlorine hyperfine component as $\mathrm{A}_{\mathrm{xx}}$ ' the component along the molecular $y$ axis as $A_{z y}$ and the component along the molecular $z$ axis as $A_{y z}$. In order to simplify the following discussion we will initially consider only the ${ }^{35} \mathrm{Cl}$ isotope.

The product functions $<u f v .\left.\right|^{ \pm}={ }_{\tau_{u}} \chi_{f}{ }^{ \pm} \chi_{v}$ form a set of eigenfunctions which diagonalize $\mathrm{H}_{S S}, \mathrm{H}_{\mathrm{Q}}^{\mathrm{N}}$ and $\mathrm{H}_{\mathrm{Q}}^{\mathrm{Cl}}$ (excluding $\mathrm{H}_{Q}^{\mathrm{Cl}}(\eta)$ ). $\tau u^{\prime} \chi_{f}^{ \pm}$and $\chi_{v}$ are the electron, chlorine nuclear and nitrogen spin. functions respectively. $u$ and $v$ correspond to $x, y$ and $z$ while $\chi_{f}^{ \pm}$take on values $+3 / 2,-3 / 2,+1 / 2$, and $-1 / 2$. With this choice of basis functions, only $\mathrm{H}_{\mathrm{HF}}^{\mathrm{N}}, \mathrm{H}_{\mathrm{HF}}^{\mathrm{Cl}}$ and the parameter containing the $\mathrm{Cl}$ field gradient asymetry, ${ }_{\mathrm{Q}}^{\mathrm{Cl}}(\eta)$, are off-diagonal in the Hamiltonian matrix. An energy level diagram appropriate to the $\tau_{\mathrm{y}} \rightarrow \tau_{\mathrm{z}}$ transition in 8-chloroquinoline is given in Figure 29. with the zern field energies arranged in the order $\mathrm{E}_{z}>\mathrm{E}_{\mathrm{y}}>\mathrm{E}_{\mathrm{x}}$. The result of $\mathrm{H}_{Q}^{\mathrm{Cl}}(\eta)$ is to mix the states $<\left.u f v\right|^{ \pm}$differing in $f$ by \pm 2 ; thus, $\eta$ cannot give intensity to "forbidden" transitions between the different. spin manifolds. However, sinse $H_{Q}^{C l}(\eta)$ ooupleo otatco that are also cuupled lo states in a ditferent electron spin manifold by $\mathrm{H}_{\mathrm{HF}}^{\mathrm{Cl}}$, $\eta$ may give risc to small frequency $(\sim 10 \mathrm{kHz})$ shifts and intensity variations via the interaction with $\mathrm{H}_{\mathrm{HF}}^{\mathrm{Cl}}$. Since these variations are very small, $\eta$ cannot be obtained $r \in l i-$ ably from our data. Therefore, the ${ }^{35} \mathrm{Cl}$ first order nuclear quadrupole' splitting between $<z 3 /\left.2\right|^{ \pm}$and $<z 1 /\left.2\right|^{ \pm}$, or $<y 3 /\left.2\right|^{ \pm}$and $<\mathrm{Y} 1 /\left.2\right|^{ \pm}$will be written in terms of one parameter ${ }^{35} \mathrm{Q}^{\prime}$, by defining 

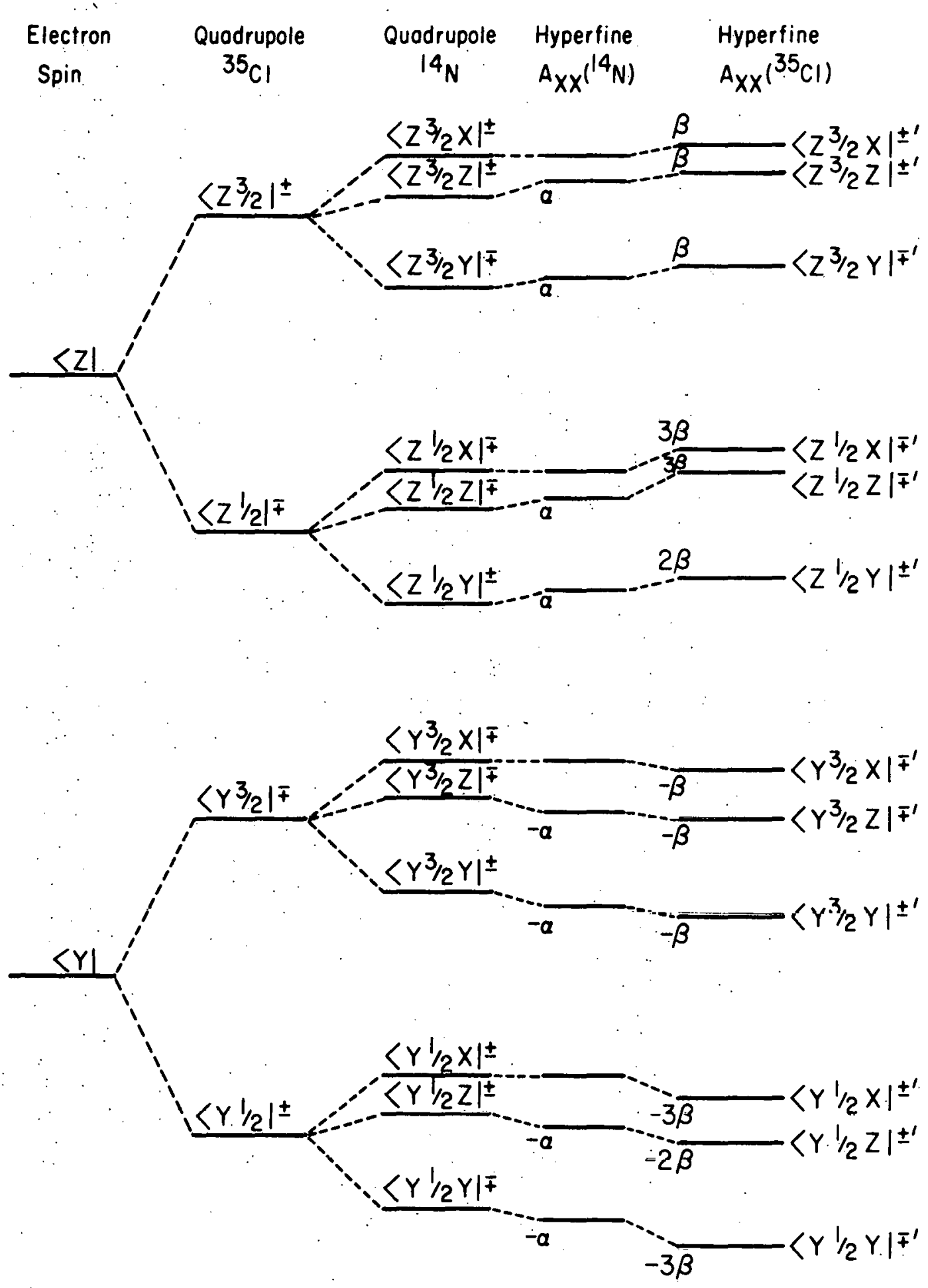

$X B L 7012-7273$

Figue 29

Energy Level Diagram for the $\tau_{z}$ and $\tau_{y}$ Triplet Manifolds. 


$$
{ }^{35} Q^{\prime}=e^{2} \mathrm{qQ}\left({ }^{35} \mathrm{Cl}\right)(1+2 / 3)^{1 / 2} .
$$

In the case of the nitrogen quadrupole interaction it has been shown ${ }^{26}$ that in the approximation $A_{y y}(N), A_{z z}(N)=0, e^{2} q Q\left({ }^{14} N\right)$ and $\eta$ cannot be obtained separately therefore, we shall use the meaoured parameter $v_{\text {- }}$ with the assumption that both the nitrogen asymetry parameter, $\eta$, and the nitrogen quadrupole coupling constant, e $e^{2} q\left({ }^{14} N\right)$, are positive.

The principal result of $\mathrm{H}_{\mathrm{HF}}^{\mathrm{N}}$ acting as a perturbation is that the levels $<\mathrm{Z} 3 /\left.2 \mathrm{Z}\right|^{ \pm},<\mathrm{Z} 3 /\left.2 \mathrm{Y}\right|^{ \pm},<\mathrm{Z} 1 /\left.2 \cdot \mathrm{Z}\right|^{ \pm}$, and $<\mathrm{Z} 1 /\left.2 \mathrm{Y}\right|^{ \pm}$shift to a higher energy by an amount $a=A_{x x}{ }^{2}(N) /(Z-Y)$ and the levels $<\mathrm{Y} 3 /\left.2 \mathrm{Z}\right|^{ \pm},<\mathrm{Y} 3 /\left.2 \mathrm{Y}\right|^{ \pm},<\mathrm{Y} 1 /\left.2 \mathrm{Z}\right|^{ \pm}$, and $<\mathrm{Y} 1 /\left.2 \mathrm{Y}\right|^{ \pm}$shift to lower energies by an equal amount (cf. Figure 29). Similarly, the result of $\mathrm{H}_{\mathrm{HF}}^{\mathrm{Cl}}$ is the mix the states in the $\tau_{\mathrm{y}}$ and $\tau_{z}$ manifolds. The order of the plus and minus sign, $<1^{ \pm}$corresponds to the components of Kramers pair which are not split in zero field. Table 6 lists the states mixed . under these perturbations. Because $\mathrm{H}_{\mathrm{HF}}^{\mathrm{Cl}}$ mixes states involving two or more basis states in the same electron spin manifold, $\mathrm{H}_{\mathrm{HF}}^{\mathrm{Cl}}$ cannot be adequately treated by first order perturbation theory. Therefore computer diagonalization of the Hamiltoninn matrix waś employed. It was l'ound that under the perturbation $\mathrm{H}_{\mathrm{HF}}^{\mathrm{Cl}}$, the states shifted to a first approximation by energies related to multiples of $\beta=A_{X X X}{ }^{2}(C I) \rho(Z-Y)$. An analysis of the problem revealed that this approximation was good to within $0.3 \mathrm{MHz}$ over a reasonable range of $\mathrm{A}_{\mathrm{Xx}}(\mathrm{Cl})$ values. We should 
emphasize that this is only an approximation but is empirically* verified. Since we resolve no splittings in the spectra directly attributable of $\mathrm{H}_{\mathrm{HF}}^{\mathrm{Cl}}$, the $\beta$ 's are employed to treat $\mathrm{H}_{\mathrm{HF}}^{\mathrm{Cl}}$ as a "pseudo" first order perturbation.

From an inspection of the states mixed by $\mathrm{H}_{\mathrm{HF}}^{\mathrm{N}}$ and $\mathrm{H}_{\mathrm{HF}}^{\mathrm{Cl}}$ (See table 6) four types of transition between the electron spin manifolds can be classiffed using the electron magnetic dipole moment transition operator. These can be described as: (a) electron spin transitions only, (b) simultaneous electron and nitrogen spin transitions, (c) simultaneous electron and chlorine spin transitions, and (d) simultaneous electron, nftrogen and chlorine spin transitions. The approximate transition energies are given in Table 5 in terms of the spin Hamiltonian parameters. It should be noted that with the approximation that both the chlorine and nitrogen in-plane hyperfine elements can be neglected, the $\tau_{x}$ manifold is not mixed with $\tau_{y}$ or $\tau_{z}$; thus, the basis functions $|u f v\rangle^{ \pm}$are not perturbed. The primed states in the tables and figures correspond to the perturbed basis functions. With the above approximation it is immediately apparent from table 5 that all parameters except. $A_{x x}(C l)$ can be obtained easily with reasonable accuracy. For example, $A_{x x}(N)$ can be estimated from the energy difference between the transitions $<Y 3 /\left.2 X\right|^{\prime} \rightarrow$ $<\mathrm{Z} 3 /\left.2 \mathrm{X}\right|^{\prime}$ and the degenerate pair $<\mathrm{Y} 3 /\left.2 \mathrm{Z}\right|^{\prime}:<\mathrm{Y} 3 /\left.2 \mathrm{Y}\right|^{\prime} \rightarrow<\mathrm{Z} 3 /\left.2 \mathrm{Z}\right|^{\prime}:$ $<\mathrm{Z} 3 /\left.2 \mathrm{Y}\right|^{*}$ while $v_{-}\left({ }^{i 4} \mathrm{~N}\right)$ can be obtained to a first approximation from * The use of empirical connotes the observation of the shifts resulting in the computer diagonalization of the Hamiltonian matrix aver a range of $A_{x x}(C l)$ values. 
the difference in the transition energies $<Y 3 /\left.2 \mathrm{Z}\right|^{\prime} \rightarrow<\mathrm{Z} 3 /\left.2 \mathrm{Y}\right|^{\prime}$ and $<\mathrm{Y} 3 /\left.2 \mathrm{Y}\right|^{\prime} \rightarrow<\mathrm{Z} 3 /\left.2 \mathrm{Z}\right|^{\prime}$. This region of the electron spin transitions $\tau_{y} \rightarrow \tau_{z}$ is shown in Figure 27 . In a similar fashion the ${ }^{35} \mathrm{Cl}$ field gradient parameter ${ }^{3}{ }^{5} \mathrm{Q}$ ' can be estimated from transitions associated with either the $\tau_{x} \rightarrow \tau_{z}$ or $\tau_{y} \rightarrow \tau_{z}$ manifolds. For instance, the transitions $<\mathrm{XI} / 2 \mathrm{X}|\rightarrow<\mathrm{Z} 3 / 2 \mathrm{X}|$, and $<\mathrm{X} 3 / 2 \mathrm{X} \mid \rightarrow<\mathrm{ZI/2X}$, or

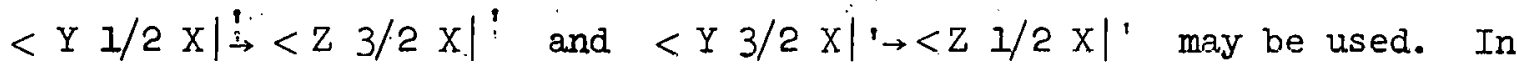
fact all parameters except $A_{x x}(C l)$ can be obtained from many different combinations of transitions as shown in Table 5. Table 7 lists the values and standard deviations obtained by averaging many of the different combinations possible in Table 5. The difficulty in obtaining an accurate measurement of $A_{x x}(C l)$ is reflected in the large error assigned to its value.

As in the cases of quinoline, ${ }^{25,26}$ quinoxaline 25 and 2,3 -dichloroquinoxaline ${ }^{2 !}$ optically detected ENDOR is predicted in 8-chloroquinoline. The intensity of the ENDOR transitions can be accounted for solely in terms of the electron magnetic dipole transition operator. Thus, ENDOR is expected between nuclear levels in only the: $\tau_{y}$ and $\tau_{z}$ manifolds since within our approximations, the $\tau_{x}$ manifold is not mixed with other electron spin states. 'l'hree different types of ENDOR are predicted and can be described as: (a) nitrogen spin transitions only, (b) chlorine spin transitions only, and (c) simultaneous chlorine and nitrogen spin transitions. In addition the ENDOR transitions should be associated with specific microwave transitions between the $\tau_{y} \rightarrow \tau_{z}$ or $\tau_{x} \rightarrow \tau_{z}$ manifolds. Table 8 lists the observed ENDOR transitions and 
assigns each of them to one of the above classifications in addition to the microwave transitions with which they are associated. Indeed all three types are observed and excellent agreement between the transition energies and associated microwave transitions is obtained. These data provide a sensitive test of the uniqueness of the assignments made in Table 5 and the parameters used to obtain the transition energies.

Although we have dealt specifically with ${ }^{35} \mathrm{Cl}$, we can explain and assign transitions associated with ${ }^{37} \mathrm{Cl}$ nuclear quadrupole and nuclear electron hyperfine interactions. The observed microwave transitions and assignments are listed in Table 9 along with the ENDOR transitions for the low frequency $\tau_{\mathrm{y}} \rightarrow \tau_{\mathrm{z}}$ microwave transitions. Naturally the intensity of these transitions are a factor of three lower than the corresponding ${ }^{35} \mathrm{Cl}$ transition because of their relative isotopic abundance. Although the difference between the ${ }^{35} \mathrm{Cl}$ and ${ }^{37} \mathrm{Cl}$ nuclear quadrupole moment is clearly observed, the difference in the nuclear magnetic moment cannot be observed since the splittings arising from the chlorine hyperfine interaction is not sufficiently resolved.

The value of the chlorine coupling constant $e^{2} q Q\left({ }^{35} \mathrm{Cl}\right)\left(1-\eta^{2} / 3\right)^{1 / 2}=$ 68.4 $\mathrm{MHz}$ is approximately the same as that reported for the ground state of 6-chloroquinoline $(69.256 \mathrm{MHz})$ and 7 -chloroquinoline $(69.362 \mathrm{MHz}), 79,80$ indicating that in the $\pi \pi^{*}$ triplet state the electron environment of the chlorine nucleus is not changed significantly from that of the ground state.

Although our analysis of the observed spectra:accounts for the major features of the data, there are several minor discrepancies which 
cannot be understood within our approximations* First, it appears that we must consider finite $A_{y y}(N), A_{z z}(N), A_{y z}(C l)$ and $A_{z y}(C l)$ values to fit all transition energies more accurately and to account for the line widths. Secondly, at maximum power (cf. Figure $l(f)$ ), six pairs of satellites appear in the $\tau_{\mathbf{x}} \rightarrow \tau_{\mathbf{z}}$ multiplet centered at $\sim 3.339,3.367$, $3.373,3.398,3.404$ and $3.431 \mathrm{GHz}$. These satellites may be due to ${ }^{13} \mathrm{C}$ or ${ }^{1} \mathrm{H}$ interactions or alternatively guest-host interactions. The possibillties should be distinguishahle by isotope substitution and/or small Zeeman perturbations.

In order to find out if the observation of the interaction of the chlorine nuclei is generally observable, we repeated the experiments of Tinti ${ }^{23}$ et al. on 2-3 dichloroquinoxaline. The power dependence of the $\tau_{y} \rightarrow \tau_{z}$ transition is shown in Figure 30. As can be seen, at minimum power (Figure 30a) the resonance shows only the structure due to the nitrogen hyperfine and quadrupole interactions. As the power is increased (Figures $30 \mathrm{~b}$ and $30 \mathrm{c}$ ), the chlorine satellites split approximately $35 \mathrm{MHz}$ from the center section are observable and increase in intensity, unti.l with maximum power ( 100 mWat.t.s) the chlorine satellitco arc almost as strong as the center or "allowed" section and transitions split approximately $70 \mathrm{MHz}$ from the center section due to simultaneous electron and two chlorine spin transitions are observed. In addition ${ }^{35} \mathrm{Cl}$ and ${ }^{37} \mathrm{Cl}$ ENDOR resonances were observed while saturating the $\tau_{y} \rightarrow \tau_{z}$ multiplet. * Since the completion of this experiment the weak emission from a second site of 8 -chloroquinoline in durene has been identified. ${ }^{36}$ The zero field splitting for the two sites are different $(\Delta \mathrm{D}=2.4 \mathrm{MHz}$ and $\triangle \mathrm{E}=150 \mathrm{MHz})$ which may increase the experimental line width and perhaps partially explain the anomalous power dependence of the ESR transitions. 

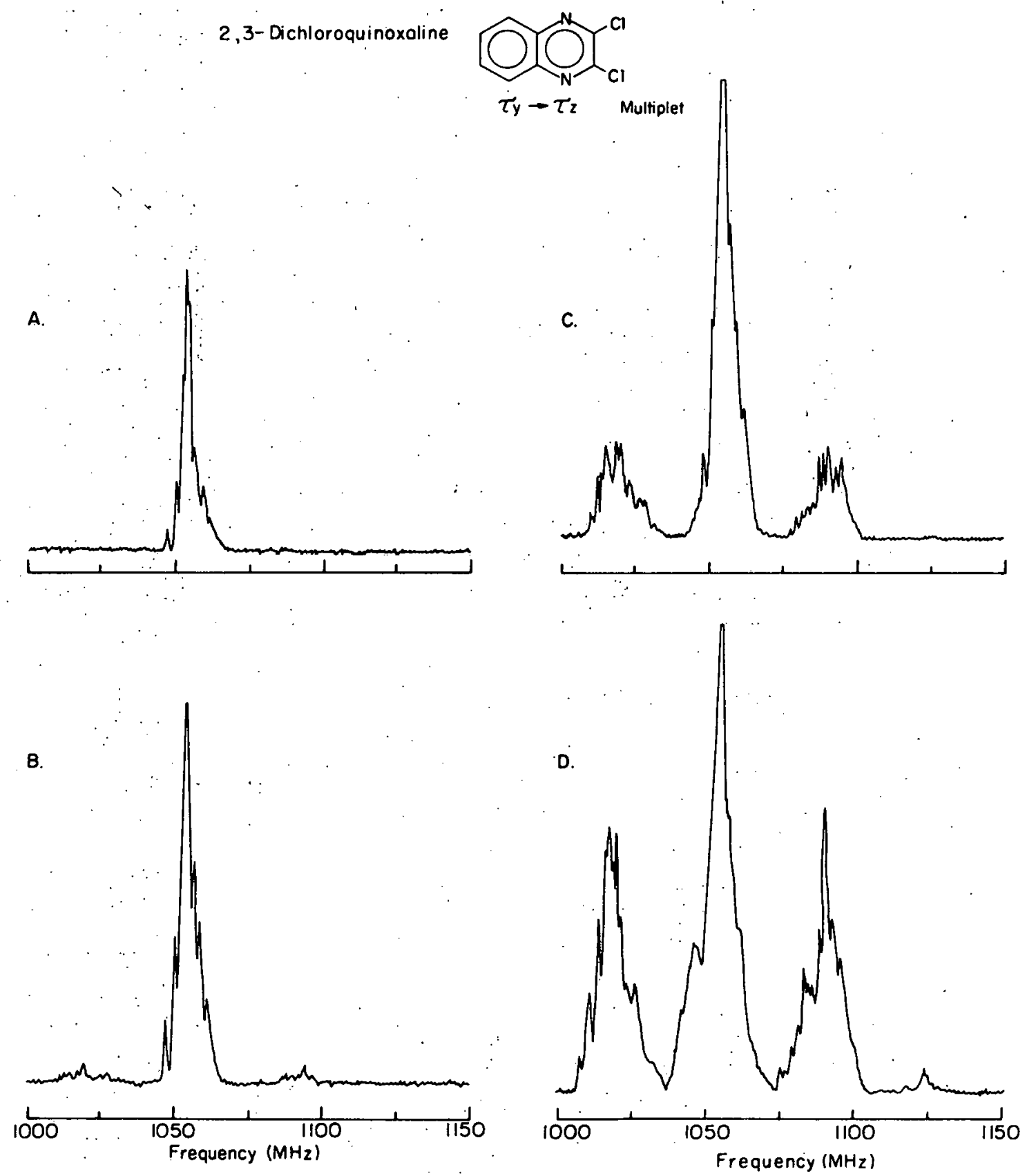

Figure 30

ODMR Spectra of $\tau_{y} \rightarrow \tau_{z}$ Multiplet of $2-3$. Dichloroquinoxaline 
No attempt was made to make a quantitative analysis of the spectra since there are two chlorine nuclei and two nitrogen nuclei in addition to the triplet electrons. Since this system has a total spin of 6 , it is not a Kromers doublet and therefore, a $432 \times 432$ matrix would have to be diagonalized in order to fit the spectra. However, the two lines in the center section of the spectra that could not. be assigned and the small splitting observed in the ${ }^{14} \mathrm{~N}$ FNDOR (see Reference 24 ) are moot likely due to the hyperfine interaction of the chlorine nuclei. 
Table 5

Energies and assignments of observed frequencies involving electron. ${ }^{14} \mathrm{~N}$, and ${ }^{35} \mathrm{Cl}$ transitions

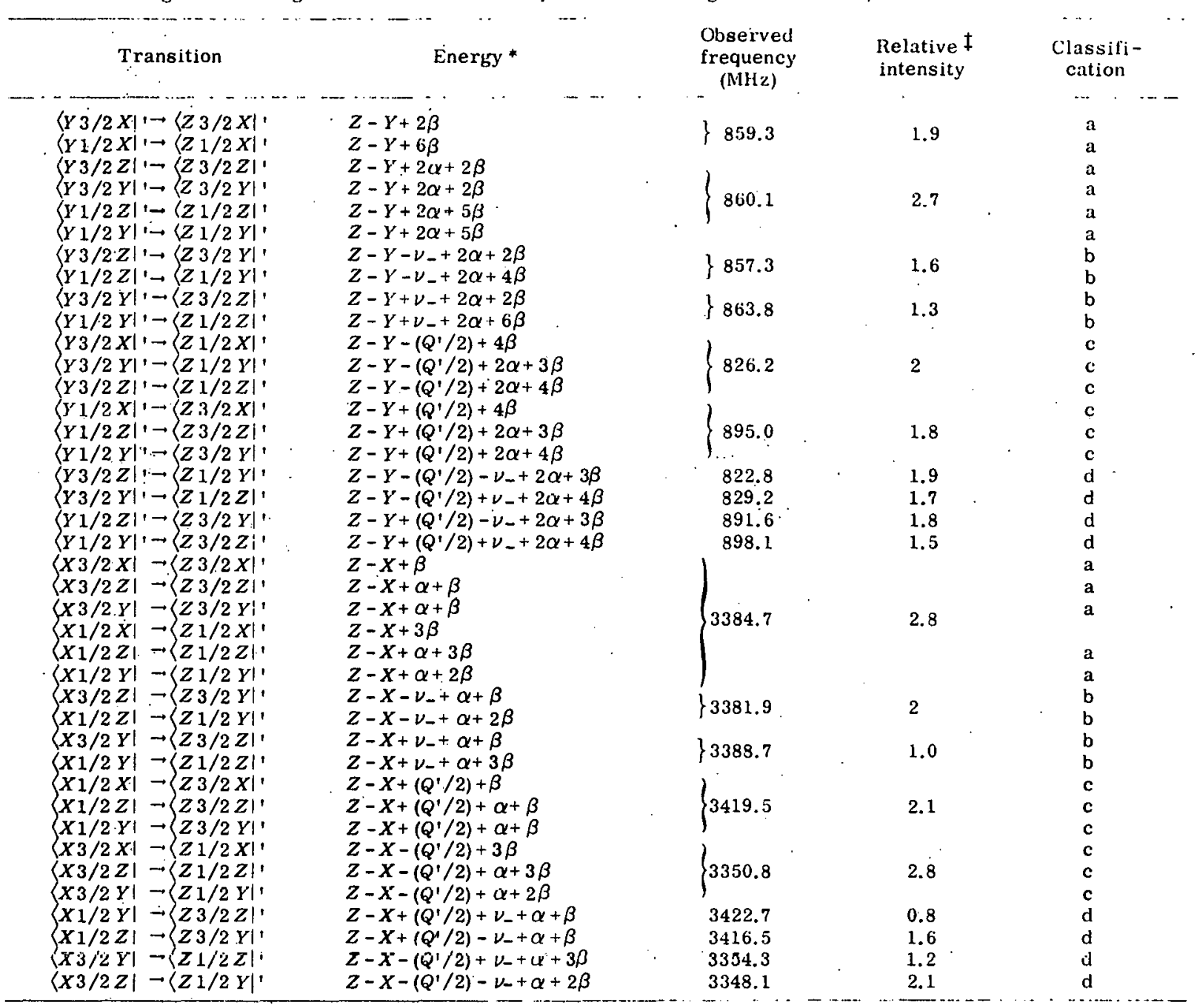

$\left.* \nu_{-}=3 e^{2} q Q\left({ }^{14} \mathrm{~N}\right)(1-\eta / 3) / 4 ; Q^{\prime}=e^{2} q Q{ }^{35} \mathrm{Cl}\right)\left(1-\eta^{2} / 3\right)^{1 / 2}$.

$¥$ Relative within the same classification only.

a Electron spin transition.

b Simultaneous electron and nitrogen spin transition.

c Simultaneous electron and chlorine spin transition.

d Simultaneous electron, nitrogen, and chlorine spin transition. 


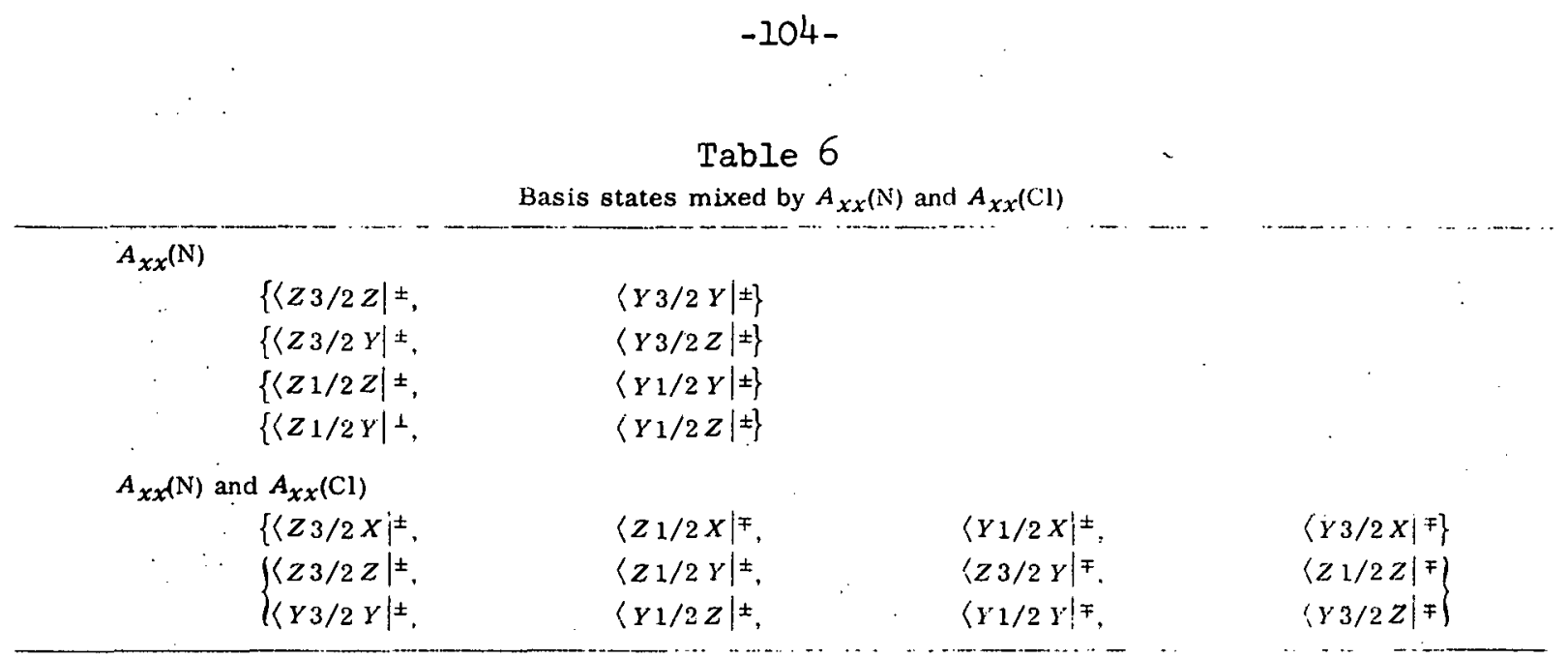

I indicate Kramers pairs.

Table 7

\begin{tabular}{|c|c|c|c|c|c|}
\hline Parameter & $\begin{array}{l}\text { Value } \\
(\mathrm{MHz})\end{array}$ & . & $\begin{array}{l}\text { Standard deviation } \\
\text { (MHz) }\end{array}$ & & $\begin{array}{l}\text { Number of combinations } \\
\text { used frnm tables } 1 ; 1\end{array}$ \\
\hline$x$ & $-1970.0^{a j}$ & & \pm 0.5 & $\therefore$ & 7 \\
\hline$Y:$ & $555.5^{\mathrm{a})}$ & & \pm 0.5 & & 7 \\
\hline$z$ & $1414.5^{a)}$ & & \pm 0.5 & & 7 \\
\hline$\left|A_{x x}(\mathrm{~N})\right|$ & 19.5 & & \pm 1.0 & . & 3 \\
\hline$\left|A_{y y}(\mathrm{~N})\right|$ & $b$ & & - & & - \\
\hline$\left|A_{z z}(\mathrm{~N})\right|$ & b & & - & & - \\
\hline$\left|A_{x x}(\mathrm{Cl})\right|$ & 15 & & $-10 .+15$ & & 12 \\
\hline$\left|A_{y z}(C)\right|$ & b & & - & & - \\
\hline$\left|A_{z y}(\mathrm{Cl})\right|$ & b & & - & & - \\
\hline$\left|\nu_{-}(\mathrm{N})\right|$ & 3.2 & & \pm 0.2 & & 20 \\
\hline$\left|Q^{\prime}\left({ }^{35} \mathrm{Cl}\right)\right|$ & 68.4 & & \pm 0.6 & & 17 \\
\hline
\end{tabular}

a) Obtained from the convention $x+y+z=0$. and assuming the same order of the zero field levels as in quinoline.

b) Date does not provide reliable values. 


\section{Table 8}

Energies and assignments of $14 \mathrm{~N}$ and $35 \mathrm{Cl}$ ENDOR

\begin{tabular}{|c|c|c|c|c|c|}
\hline $\begin{array}{c}\text { ENDOR * Transition } \\
\vdots\end{array}$ & $\begin{array}{c}\text { Nicrowave } \\
\text { transition } \\
\text { saturated } \\
\text { (MHz) }\end{array}$ & $\begin{array}{l}\text { ENDOR } \\
\text { energy }\end{array}$ & $\begin{array}{l}\text { Observed } \\
\text { ENDOR } \\
\text { frequency } \\
(\mathrm{MHz})\end{array}$ & $\begin{array}{l}\text { Relative } \\
\text { intensity }\end{array}$ & $\begin{array}{l}\text { Classifi- } \\
\text { cation }\end{array}$ \\
\hline$\left\langle Z 3 /\left.2 Y\right|^{\prime} \div\langle Z 3 / 2 Z|\right.$ & & $\nu_{-}$ & & & $\mathbf{a}$ \\
\hline$\left\langle Z_{1} /\left.2 Y\right|^{\prime} \rightarrow\left\langle Z_{1 / 2} Z^{\prime}\right.\right.$ & & $\nu_{-}+\beta$ & & & a \\
\hline $\left.\begin{array}{l}\left\langle Y 3 /\left.2 Y\right|^{\prime} \rightarrow\left\langle Y 3 /\left.2 Z\right|^{\prime}\right.\right. \\
\left\langle Y 1 /\left.2 Y\right|^{\prime} \rightarrow\left\langle Y 1 /\left.2 Z\right|^{\prime}\right.\right.\end{array}\right\}$ & 857.3 & $\begin{array}{l}\nu_{-} \\
\nu_{-}+\beta\end{array}$ & 3.2 & 10 & $\begin{array}{l}\mathbf{a} \\
\mathbf{a}\end{array}$ \\
\hline$\left\langle Z_{1} 1 / 2 X\right| 1-\langle Z 3 / 2 X|$ & & $\left(Q^{\prime} / 2\right)-2 \beta$ & .. & & b \\
\hline$\left\langle Z 1 /\left.2 Z\right|^{\prime} \rightarrow\left\langle Z 3 /\left.2 Z\right|^{\prime}\right.\right.$ & & $\left(Q^{\prime} / 2\right)-2 \beta$ & . & & b \\
\hline$\left\langle Z 1 /\left.2 Y\right|^{\prime}-\left\langle Z 3 /\left.2 Y\right|^{\prime}\right.\right.$ & & $\left(Q^{\prime} / 2\right)-\beta$ & & & b \\
\hline$\{r 1 / 2 X \mid \cdot \rightarrow\langle Y 3 / 2 X|$ & 826.2 & $\left(Q^{\prime} / 2\right)+2 \beta$ & 33.5 & 11 & b \\
\hline$\left\langle\mathrm{Y}^{\prime} \mathrm{L} / 2 Z\right| \cdot \rightarrow\left\langle\mathrm{Y}^{\prime}: 3 / 2 \geq\right|$ & 822.8 & $\left(Q^{\prime} / 2\right)+\beta$ & .33 .9 & 2 & $b$ \\
\hline$\left\langle Y 1 /\left.2 Y\right|^{\prime} \rightarrow\left\langle Y 3 /\left.2 Y\right|^{\prime}\right.\right.$ & $829.2 ; 826.2$ & $\left(Q^{\prime} / 2\right)+2 \beta$ & 33.5 & $2: 11$ & b \\
\hline$\left\langle Z 1 /\left.2 Z\right|^{\prime} \rightarrow\left\langle Z 3 /\left.2 Y\right|^{\prime}\right.\right.$ & & $\left(Q^{\prime} / 2\right)-\nu_{-}-2 \beta$ & & & $\mathrm{c}$ \\
\hline$\left\langle Z 1 /\left.2 Y\right|^{\prime}-\left\langle Z 3 /\left.2 Z\right|^{\prime}\right.\right.$ & & $\left(Q^{\prime} / 2\right)-\nu_{-}-\beta$ & & & c \\
\hline$\left\langle Y^{\prime} 1 /\left.2 Z\right|^{\prime} \rightarrow\left\langle Y 3 /\left.2 Y\right|^{\prime}\right.\right.$ & $829.2 ; 826.2$ & $\left(Q^{\prime} / 2\right)-\nu_{-}+\beta$ & 31.5 & $2 ; 2$ & c. \\
\hline$\left\langle Y^{\prime} 1 / 2 Y Y^{\prime} \rightarrow\left\langle Y^{\prime} 3 /\left.2 Z\right|^{\prime}\right.\right.$ & $822.8 ; 826.2$ & $\left(Q^{\prime} / 2\right)+\nu_{-}-2 \beta$ & 37.5 & $7 ; 2$ & c \\
\hline
\end{tabular}

* We do not see ENDOR while saturating microwave transition in the $\tau_{x} \rightarrow \tau_{z}$ multiplet. Since ENDOI is not predicted in the $\tau_{x}$ manifold and presumably some relaxation mechanism interferes in the $\tau_{z}$ manifold. the observed

ENDOR is assigned to the $\tau_{y^{\prime}}$ manifold.
$\nu_{-}=3 e^{2} q Q\left({ }^{14} \mathrm{~N}\right)(1-\eta / 3) / 4 ; Q^{\prime}=e^{2} q Q\left({ }^{35} \mathrm{Cl}\right)\left(1-\eta^{2} / 3\right)^{1 / 2}$.

a $14 \mathrm{~N}$ nuclear spin transition.

b $35 \mathrm{Cl}$ nuclear spin transition.

c Simultaneous $14 \mathrm{~N}$ and $35 \mathrm{Cl}$ nuclear spin transition. 
Table $9 a$

Energies and assignments of observed frequencies involving electron, ${ }^{14} \mathrm{~N}$, and ${ }^{37} \mathrm{Cl}$ transitions

\begin{tabular}{|c|c|c|c|c|c|c|c|}
\hline 'Transition & Energy * & & $\begin{array}{l}\text { Ubserved } \\
\text { Irequency } \\
(\mathrm{MH} \%)\end{array}$ & $\begin{array}{l}\text { Relative } \\
\text { intensity }\end{array}$ & & $\begin{array}{c}\text { Classifi } \\
\text { cation }\end{array}$ & \\
\hline$\langle Y 3 / 2 X|\cdot \rightarrow| Z 1 / 2 X|$ & $Z-Y-\left(Q^{\prime} / 2\right)+4 \beta$ & & - & $-\cdot$. & & $a$ & $\cdots$ \\
\hline$\langle Y 3 / 2 Y| \cdot \rightarrow\langle Z 1 / 2 Y|$ & $Z-Y-\left(Q^{\prime} / 2\right)+2 \alpha+3 \beta$ & & 833.0 & 1.5 & & a & \\
\hline$\langle y 3 / 2 Z| \cdot-\left\langle Z_{1 / 2} z\right|$ & $Z-Y-\left(Q^{\prime} / 2\right)+2 \alpha+4 \beta$ & & & & & $\mathfrak{a}$ & \\
\hline$|Y 1 / 2 X| \cdot \rightarrow Z 3 / 2 X \mid$ & $Z-\gamma+\left(Q^{*} / 2\right)+4 \beta$ & & & & & a & . \\
\hline$\left\langle Y_{1 / 2 Z \mid 1} \rightarrow\left\langle Z 3 /\left.2 Z\right|^{\prime}\right.\right.$ & $Z-Y+\left(Q^{\prime} / 2\right)+2 \alpha+3 \beta$ & . & 887.9 & 1.3 & & a & \\
\hline$i Y 1 /\left.2 Y\right|^{\prime} \rightarrow\left\langle Z 3 /\left.2 Y\right|^{\prime}\right.$ & $Z-\gamma+\left(Q^{\prime} / 2\right)+2 \alpha+4 \beta$ & & & & & a & \\
\hline$\{Y 3 / 2 Z|\rightarrow| Z 1 / 2 Y \mid$ & $Z-Y-\left(Q^{\prime} / 2\right)-\nu_{-}+2 \alpha+3 \beta$ & & 829.2 & 1.7 & & b & \\
\hline$\left\langle Y 3 /\left.2 y\right|^{\prime}-\left\langle Z 1 /\left.2 Z\right|^{\prime}\right.\right.$ & $Z-Y-\left(Q^{\prime} / 2\right)+\nu_{-}+2 \alpha+4 \beta$ & & 835.9 & 1.1 & & b & \\
\hline$\left\langle Y 1 /\left.2 Z\right|^{\prime} \rightarrow\left\langle Z 3 /\left.2 Y\right|^{\prime}\right.\right.$ & $Z-Y+\left(Q^{\prime} / 2\right)-\nu_{-}+2 \alpha+3 \beta$ & & 884.1 & 1.9 & & b & \\
\hline$\therefore Y 1 / 2 Y^{\prime}|+| Z 3 / 2 Z \mid$ & $\ddot{Z}-\gamma+\left(Q^{\prime} / 2\right)+\nu_{-}+2 \alpha+4 \beta$ & & 891.6 & 1.5 & & h & \\
\hline$\langle X 1 / 2 X|\rightarrow| Z 3 / 2 X|$ & $Z-X+\left(Q^{\prime} / R\right)+\beta$ & & & & & a & \\
\hline$\langle x|/ \angle Z| \rightarrow ! \angle 3 / 2 \angle|$ & $Z-X+\left(Q^{+} / 2\right)+\alpha+\beta$ & & 3412.0 & 1.9 & & a & \\
\hline$\left\{X\left|/ 2 \gamma^{\prime}\right| \cdots|Z 3 / 2 Y|\right.$ & $Z=X+\left(Q^{\prime} / 2\right)+\alpha+\beta$ & & & & & a & \\
\hline$(x|3 / 2 x| \rightarrow|z 1 / 2 x|$ & $Z-X-\left(Q^{\prime} / 2\right)+3 \beta$ & & · & & . & a & \\
\hline$\langle X 3 / 2 Z| \rightarrow\left\langle Z 1 /\left.2 Z\right|^{\prime}\right.$ & $Z-X-\left(Q^{1} / 2\right)+\alpha+3 \beta$ & & $3: 158 \cap$ & 2.1 & & a & $\cdot$ \\
\hline$\left\langle X 3 / 2 Y|\rightarrow| Z 1 /\left.2 Y\right|^{\prime}\right.$ & $Z-X-\left(Q^{\prime} / 2\right)+\alpha+2 \beta$ & & & & & a & \\
\hline$\langle X 1 / 2 Y| \cdots\langle Z 3 / 27|$ & $Z-X+\left(Q^{\prime} / 2\right)+\nu_{-}+\alpha+\beta$ & & 3416.5 & 1.2 & & $\mathrm{~b}$ & \\
\hline $\mid X_{1 / 2 Z \mid}{ }^{\prime}\langle\langle Z 3 / 2 Y|$ & $Z-X+\left(Q^{\prime} / 2\right)-\nu_{-}+\alpha+\beta$ & & 3409.2 & 0.8 & & $\mathrm{~b}$ & \\
\hline$(X 3 / 2 Y) \cdots(Z 1 / 2 ?\}$ & $Z-X-\left(Q^{\prime} / 2\right)+\nu_{-}+\alpha \cdot 3 \beta$ & ' & 3361.5 & 0.8 & & b & $:$ \\
\hline$\langle X 3 / 2 Z| \rightarrow\langle Z 1 / 2 Y|$ & $Z-X-\left(Q^{\prime} / 2\right)-\nu_{-}+\alpha+2 \beta$ & & 3354.3 & 1.2 & & b & \\
\hline
\end{tabular}

Table 9b 


\section{B. The ${ }^{3} n \pi^{*}$ State of Pyrazine}

Since pyrazine is an example of the class of molecules in which an electron is excited from a non-bonding ( $n$ ) orbital to an anti-bonding $\left(\pi^{*}\right)$ orbital, it has been the subject of considerable interest for many

years. $81-84$ The purpose of our investigation is to gain a measure of the electron distribution in the excited $n \pi^{*}$ state of pyrazine by measuring the electron spin-spin, nitrogen hyperfine and nitrogen nuclear quadrupole interactions.

In contrast to $\pi^{*}$ triplets, where because of the Pauli Principle the unpaired electrons may not be on the same atomic centers, in $n \pi^{*}$ triplets the unpaired electrons are in orthogonal orbitals and therefore may reside on the same atomic center. The $n \pi^{*}$ triplet therefore has large one-center contributions to both the spin-orbit and zero field Hamiltonians. Experimentally, this results in both a short lifetime for the excited $n \pi^{*}$ state due to the larger spin-orbit coupling contrijution and higher frequency ESR transitions due to the larger electron spin-spin interaction.

Both of these effects have contributed to the failure to detect triplets using conventional absorption techniques. Since the sensitivity of experiments in which the absorption of energy is monitored is directly dependent on the concentration of spins, the short lifetime results in a reduction in sensitivity directly proportional to the shorter lifetime of the excited state. With ODMR on the other hand, the sensitivity is dependent on the number of photons detected per unit time which is approximately equal to the steady state population divided by 
$-108-$

the lifetime: Since, to a first approximation, the steady state population is proportional to the lifetime, the sensitivity using optical detection techniques is independent of the lifetime of the excited state.

The sample consisted of a single crystal of paradichlorobenzene (pDB) doped with $1 \%$ pyrazine $h-4$ or $d-4$ grown from the melt. The starting material was recrystallized, vacuum sublimed and zone refined. The experimental arrangement was essentially the same as that shown in Figure 16. The sample was maintained at $2.0^{\circ} \mathrm{K}$ while being irradiated with the $3100 \AA$ region of the mercury arc lamp. The 0-0 band of the pyrazine phosphorescence was detected at a $90^{\circ}$ angle to the exciting light. The microwave sweep oscillator was amplitude modulated at 40 $\mathrm{Hz}$ for the $\mathrm{D}+|\mathrm{E}|$ and $\mathrm{D}-|\mathrm{E}|$ transitions while a Hewlett-Packard mudel $3200 \mathrm{~B}$ RI oscillator was used without modulation for the $2 \mathrm{E}$ transition.

The $D+|E|$ and $D-|E|$ transitions for pyrazine-h4 and $-d 4$. are shown in Figures $3 I$ and 32 , and the power dependence of the $2 E$ transition in Figure 33. The large linewidth of the three zero field transitions is primarily due to the large hyperfine interaction. As discussed in Section III, the main features of the spectra can be explained by a serond order perturbntion treatment. The high frequency or $D+|E|$ transition (see Figures $3 I B$ and $32 B$ ) should be shifted to a higher energy by an amount $\alpha=A_{z z}{ }^{2} /\left(E_{x}-\mathbb{F}_{y}\right)$ while the $D-|E|$ transition (see Figures $31 \mathrm{~A}$ and $32 \mathrm{~A}$ ) should be shifted to lower energy by an equal amount and the $2 \mathrm{E}$ transition (see Figure 33) shifted to higher energy by an amount 2a. The measured values of $D$ and $E$ 

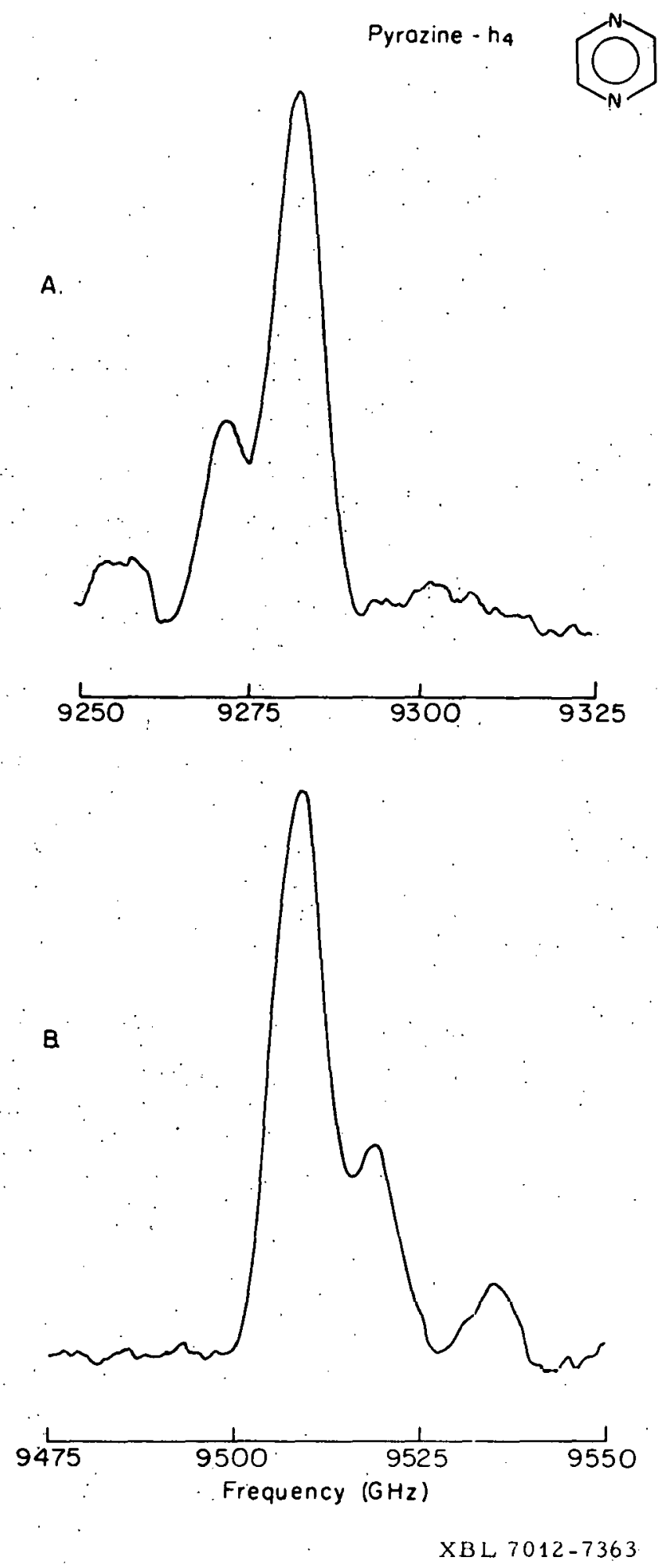

Figure 31

Pyrazine-h4 ODMR of the $D+E(a)$ and $D-E(b)$ Zero Field Transitions 
A.
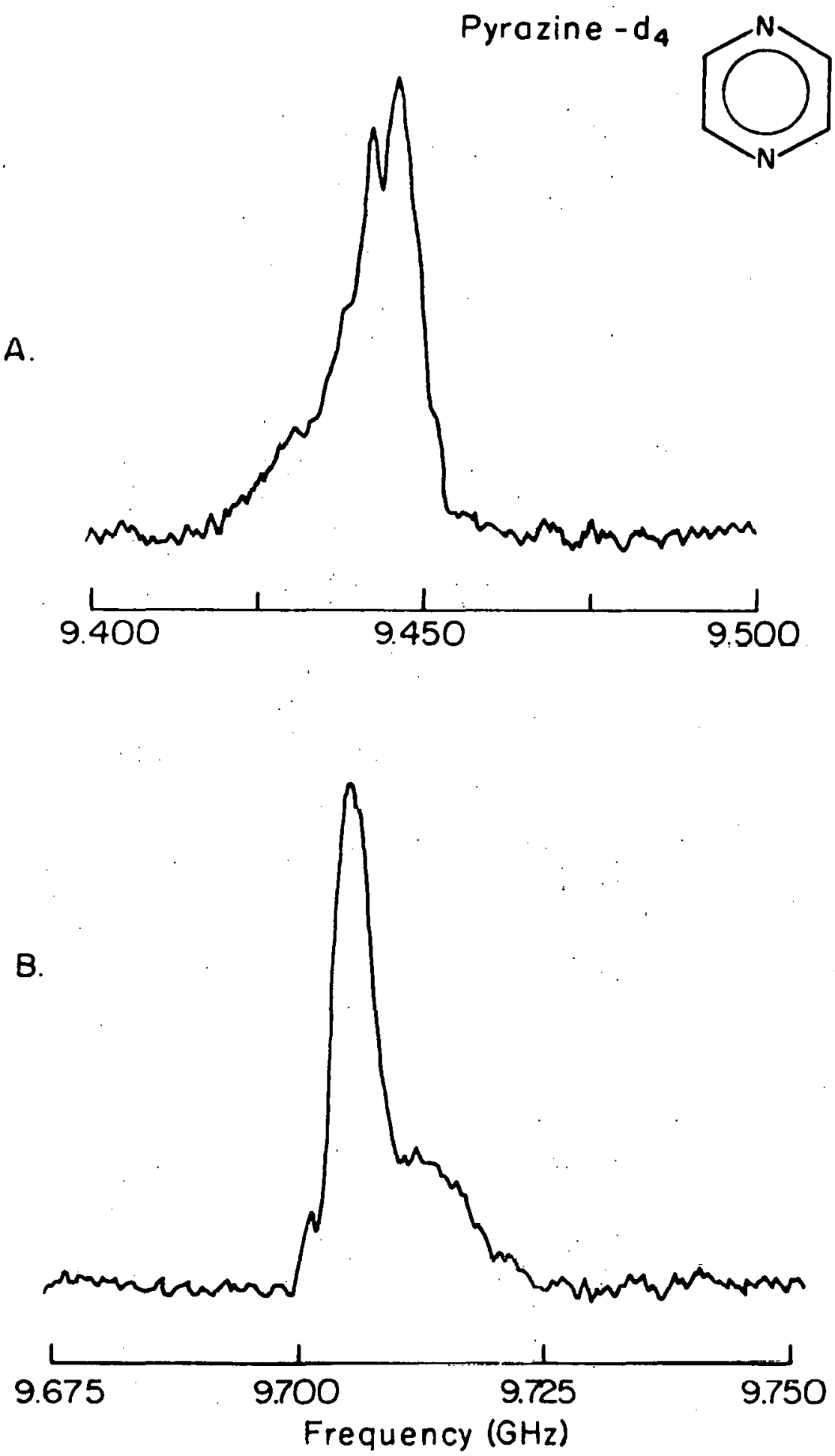

XBL $7012-7364$

H'igure 32

Pyrazine-d4 ODMR of the $D+E(a)$ and $D-E(b)$ Zero Field Transitions 
Pyrazine - $d_{4}$

$O_{N}^{N}$

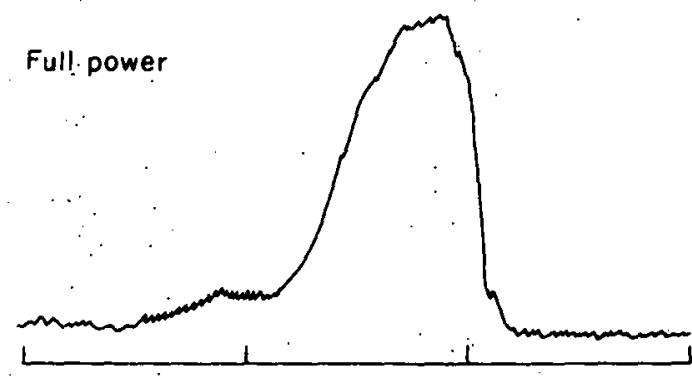

$\cdot-24 d b$
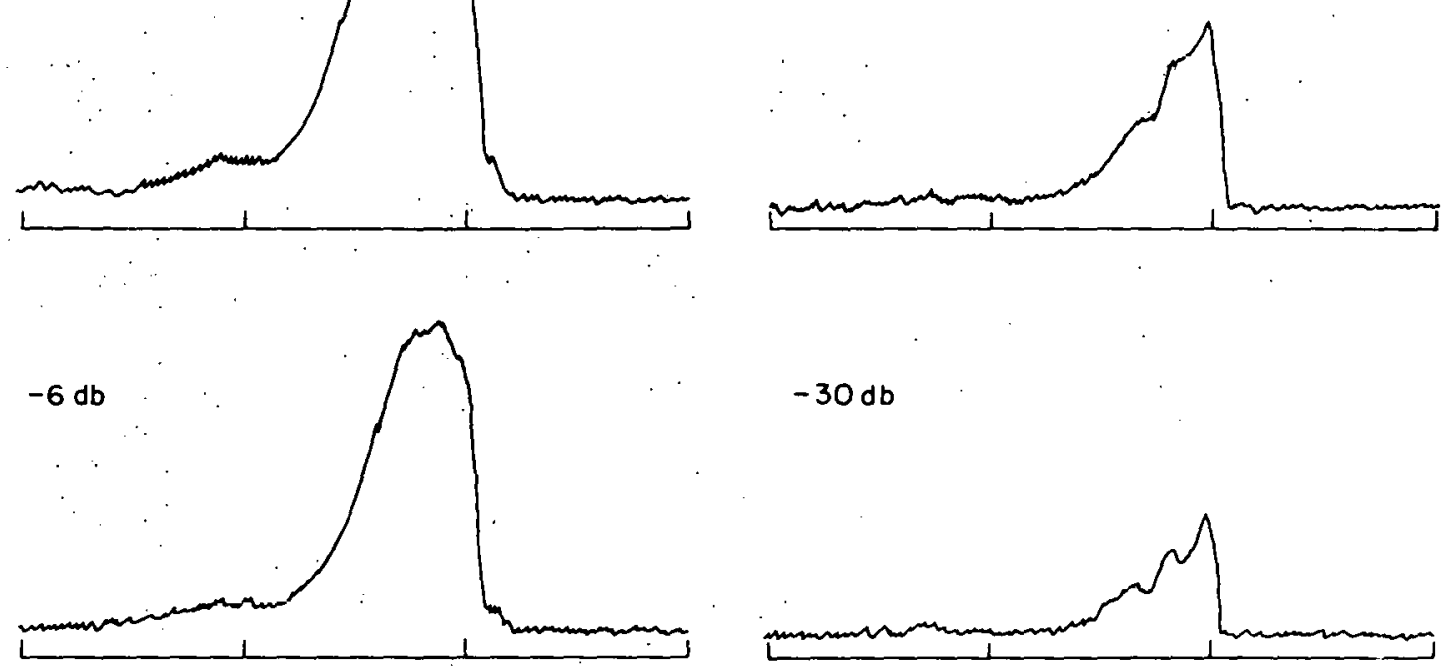

$-30 d b$
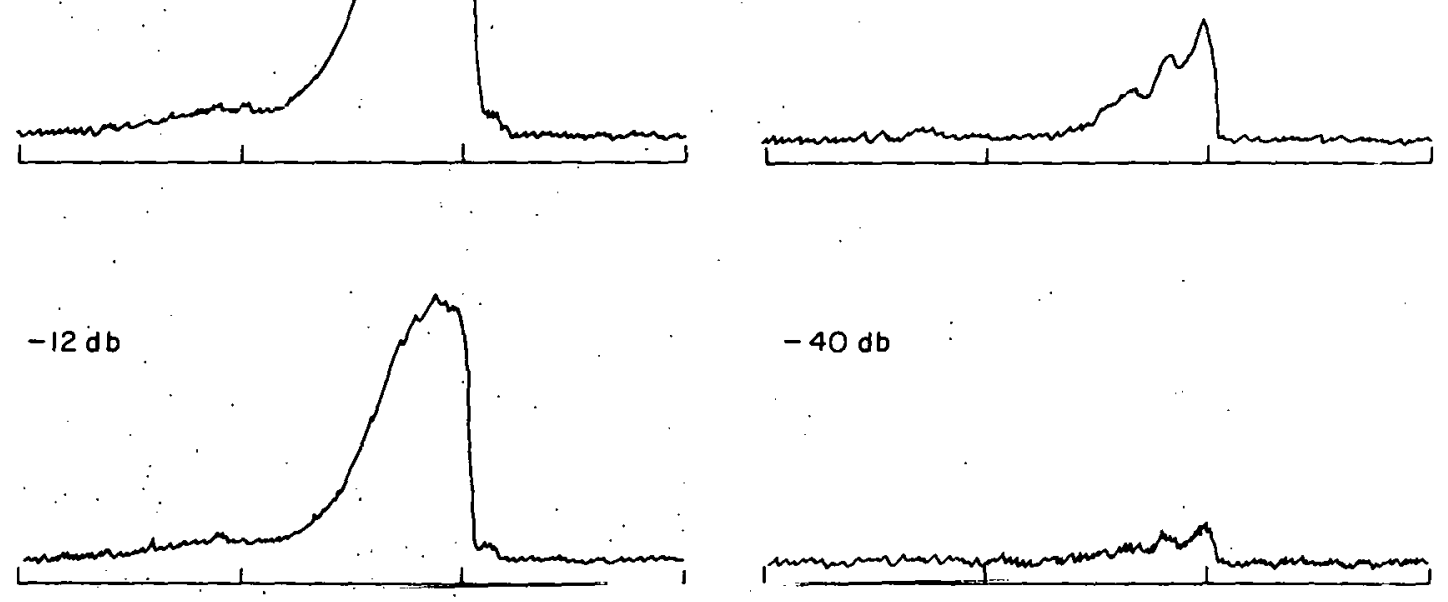

$-40 d b$
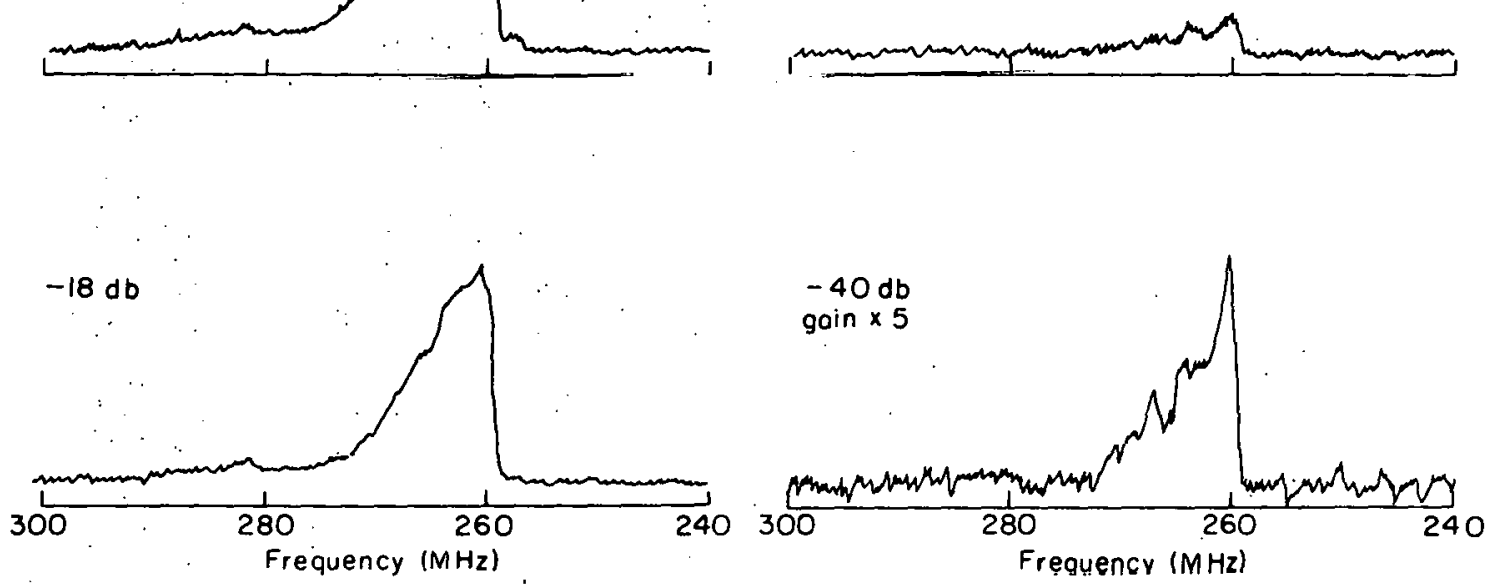

Figure 33

XBL $7012-7361$

Power Dependence of the 2E Transition of Pyrazine-d4 
for pyrazine-h4 are $|D|=9.3959 \mathrm{GHz},|\mathrm{E}|=0.1126 \mathrm{GHz}$ and for pyrazine-d4 $|\mathrm{D}|=9.5782 \mathrm{GHz}$ and $|\mathrm{E}|=0.1286 \mathrm{GHz}$.

The high field optical detection of the pyrazine-h4 ESR has been reported by Sharnoff $\mathrm{f}^{37}$ with the values $\mathrm{D}=+9.264 \mathrm{GHz}$ and $E=-0.180 \mathrm{GHz}$, while Cheng and Kwiram ${ }^{38}$ have reported the low field and zero field optical detection of the $D+|E|$ and $D-|E|$ transitions. The values they report are, for pyrazine-h4, $D= \pm 10.1698 \mathrm{GHz}$, $F=\mp 0.2155 \mathrm{GHz}$; and for pyraxille-d4, $D=+10.2043 \mathrm{iH} \bar{z}$ and $E=\mp 0.2057 \mathrm{GHz}$. In addition, Hochstrasser and Lin $^{85}$ have observëd the zero field splitting of pyrazine in the optical spectra by use of a large Zeeman field. They report the value $D=+0.3 \mathrm{~cm}^{-1}$ (9 GHz) which is consistent with the ESR studies.

The discrepancy between nur reanlt.s and Sharnoff's arc most likely due to the lower accuracy inherent in his high field experiments; while the difference between our results and those of Cheng and Kwiram are believed to be due to differences in sample preparation. Chenge and KwIram performed their experiments shortly after the crystal was grown from the melt, while our experiments were performed approximately one year after growing the crystal. The sensitivity of pDB to its recent thermal history will be discussed in more detail in the section on the ESR of that molecule, but most likely the sample used by Cheng and Kwiram contained an appreciable concentration of the triclinic (or high temperature) form of $\mathrm{pDB}$. The environmental difference between the monoclinic and triclinic forms of $\mathrm{pDB}$ are believed to account for the approximately 10\% variation in the values of $D$. 
On the basis of the optical spectra, the spacial symetry of the ${ }^{3} \pi^{*}$ state of pyrazine has been assigned as $B_{34}{ }^{86}$ in $D_{2 h}$ using an axis system with the $N-N$ axis as the $y\left(B_{1 u}\right)$ axis, the other in-plane axis as $z\left(B_{1 . u}\right)$ and the out-of-plane axis as $x\left(B_{3 u}\right)$. The three triplet levels transform as the product of the space and spin representations and therefore the total symetry of the triplet levels may be expressed as,

$$
\begin{aligned}
& \tau_{x}=B_{3 u} \times B_{3 g}=A_{u} \\
& \tau_{y}=B_{3 u} \times B_{2 g}=B_{1 u} \\
& \tau_{z}=B_{3 u} \times B_{2 g}=B_{2 u}
\end{aligned}
$$

In contrast to ESR experiments using a magnetic field, experiments at zero magnetic field do not give any information as to the sign of $D$ and E. The experiments of Cheng and Kwiram using a magnetic field have established that $D$ and $E$ must be of opposite signs. This requires that the energy levels be ordered $E_{z}<E_{y}<E_{x}$ or vice versa. A simple analysis of the electron spin-spin interaction centered on the nitrogen predicts that $E_{x}$ and $E_{y}$ are positive and $E_{z}$ negative which requires that $D$ be $>0$ and $E<0$. In addition, the excited nitrogen atom fragments are isoelectronic with the $>\mathrm{C}$ : fragment in diphenylmethylene and fluorenylidene 47 in which $D$ is $>0$ and $E<0$ using our axis system. This assignment of the order of the energy levels is further supported by Sharnoff, except that he gives the opposite signs for $D$ and $E$ with the same ordering of the energy levels. The energies of the triplet levels for pyrazine-h4 are therefore: 


$$
X=3.2446 \quad Y=3.0194 \quad Z=-6.2640 \mathrm{GHz}
$$

and for pyrazine-d4,

$$
X=3.1213 \quad Y=3.0641 \quad Z=-6.3854 \mathrm{GHz}
$$

An accurate interpretation of the zero field values is of course dependent on an $a b$ initio calculation of the excited $n \pi^{*}$ state of pyrazine. However, several conclusions can be drawn from a simple analysis of the spacial dependence of the zero field interaction for two unpaired electrons in orthagonal $\mathrm{p}$ orbitals located on the same atomic center.

The approximate equivalence of the $X$ and $Y$ triplet levels requires with this model that the electron density in the lone pair and $\pi^{*}$ orbitals on the nitrogen atoms be approximately equivalent. This would imply that the electron in the $\pi^{*}$ orbital does not spend much time on the carbon atoms.

Considering only the nitrogen one-center contributions to the zero field splitting, the smali ratio of $\mathrm{E} / \mathrm{D}$ indicates a small amount of $s$ character in the nitrogen lone pair orbital. Sternlicht, 87 on the basis of $\mathrm{sp}^{2}$ hybridization calculated a ratio of $\mathrm{E} / \mathrm{D}$ of -0.5 while the measured valuco nre for pysazint-h/, $E / D=-0.0180$ and f'or pyrazine-d4, $E / D=-0.0134$. Without any $s$ character in the lone pair orbital the value of $E$ would of course be zero.

The nitrogen hyperfine interaction has been obtained by fitting the spectra to a Hamiltonian incorporating the zero field, nitrogen hyperfine and nitrogen nuclear quadrupole terms. Due to the large line 
widths it was not possible to obtain an accurate measure of the nitrogen quadrupole coupling constant and therefore the ground state values 88 were used in fitting the spectra. The values obtained for the nitrogen hyperfine interaction are

$$
\begin{aligned}
& A_{x x}{ }^{\prime} \approx 70 \mathrm{MHz} \\
& A_{y y}=80 \mathrm{MHz} \\
& A_{z z}, \approx 30 \mathrm{MHz}
\end{aligned}
$$

It must be emphasized that these are only approximate values and subject to an error on the order of $\pm 20 \%$, but are essentially the same as those reported by Cheng and Kwiram. These values give for the isotropic component of the hyperfine interaction $a_{c}=60 \mathrm{MHz}$ and. for the: anisotropic components

$$
\begin{aligned}
& A_{x x}=10 \\
& A_{y y}=20 \\
& A_{z z}=-30
\end{aligned}
$$

The anisotropic hyperfine coupling may be interpreted with the aid of the tables given by Asycough. 89 One electron in a $p$ orbital will contribute an amount $2 \mathrm{~A}\left(\mathrm{~A}=47.8 \mathrm{MHz}\right.$ for $\left.{ }^{14} \mathrm{~N}\right){ }^{89}$ in the direction of the orbital and an amount $-A$ in the other two orthagonal directions to the anisotropic hyperfine interaction. The triplet electrons are in the $\pi^{*}$ and Ione pair orbital on the nitrogens with electron densities $\rho_{\pi}$ and $\rho_{L}$ respectively. In our axis system we may express the contribution of each of the triplet electrons to the anisotropic hyperfine components as, 


$$
\begin{aligned}
& A_{x x}=\left(2 A \rho_{\pi}-A \rho_{L}\right) \\
& A_{y y}=\left(2 A \rho_{L}-A \rho_{\pi}\right) \\
& A_{z z}=\left(-A \rho_{\pi}-A \rho_{L}\right)
\end{aligned}
$$

The spin densities $\rho_{\pi}$ and $\rho_{I}$ may be obtained by solving Equation 9 , which gives us,

$$
\begin{aligned}
& \rho_{\pi}=\frac{1}{3 A}\left(A_{x x}-A_{z z}\right) \\
& \rho_{I}=\frac{1}{3 A}\left(A_{y y}-A_{z z}\right)
\end{aligned}
$$

Substituting the measured values of the anisotropic hyperfine tensor we find that $\rho_{\pi} \approx 0.28$ and $\rho_{L}=0.35$ for both mitrogen atoms.

Hirota, Hutchison and Palmer 94 have shown that the experimental results for naphthalene bear out McLachlan's 95 theoretical prediction that the spin density of the lowest triplet state is approximately the same as the negative ion.

our results f'or the $3_{n \pi^{*}}$ state of pyrazine are consistent with this since our value for the spin density of the electron in the $\pi^{*}$ orbital on the nitrogen atoms $(0.28)$ is almost identical with the experimental value for the pyrazine anion of 0.278 .96

The theoreticall isotropic hyperfine interaction due to one electron In a nitrogen $2 \mathrm{~s}$ orbital is $1540 \mathrm{MHz} .{ }^{89}$ since this contribution should dominate over the induced polarization of the $s$ electrons by the triplet electrons in $p$ orbitals, we will assume that only the direct contribution need be considered in interpreting the observed isotropic hyperfine 
interaction. Therefore, the triplet spin density in the nitrogen 2s orbital $\rho_{s}$ is given by

$$
\rho_{s}=(60 / 1540)=0.04
$$

The coefficient of the nitrogen $2 s$ atomic orbital in the nitrogen lone pair molecular orbital is therefore equal to $(0.04)^{\frac{1}{2}}=0.20$. This gives us for the wave function of the nitrogen lone pair orbital

$$
\psi_{L}=0.20 \phi_{2 s}+0.98 \phi_{2 p_{y}}
$$

This small amount of $s$ character in the lone pair orbital is consistent with the zero field values and indicates a bent bond with an angle $>120^{\circ}$.

A quarititative estimate of the angle may be obtained by using the equation given by Higuchi ${ }^{91}$ for the bond angle

$$
\psi_{L}=\left[\frac{(1+\cos \theta)}{(1-\cos \theta)}\right]^{\frac{1}{2}} \phi_{2 s}+\left[\frac{-2 \cos \theta}{(1-\cos \theta)}\right]^{\frac{1}{2}} \cdot 3_{2 p_{y}}
$$

where $\Psi_{I}$ is the lone pair orbital and $\theta$ is the $\mathrm{C}-\mathrm{N}_{2} \mathrm{C}$ angle.

Therefore, from the measured value of the isotropic hyperfine interaction we have

$$
\left[\frac{(1+\cos \theta)}{(1-\cos \theta)}\right]^{\frac{1}{2}}=0.20
$$

which gives us an angle of $157^{\circ}$. It should be noted that calculations of the ground state of pyrazine by Clementi ${ }^{92}$ indicate a small $\mathrm{s}$ character $(\approx 15 \%)$ for the lone pair orbital using a fixed geometry with $\theta=120^{\circ}$. 
Cheng and Kwiram ${ }^{38}$ raised the question of the possibility of population inversion of the triplet levels of pyrazine. This question can of course be answered if a conventional ESR experiment can be performed. Alternatively an analysis of the change in phosphorescence intensity upon saturating the three zero field transitions will eupply the same information.

It is necessary to know the lifetimes of the individual triplet levels in order to answer this question, but in the case of pyrazine the assignment is reasonably straightforward.

$$
\text { Since the } \tau_{x} \text { level transforms as } A_{u} \text { (Eq. 8) it is symmetry }
$$
forbidden to directly couple via the spin-orbit Hamiltonian with an excited singlet state. Therefore if we neglect the triplet character of the ground state, the $\tau_{x}$ level may only gain the admixture of single singlet character necessary for electric dipole radiation to the ground state by spin-orbit coupling through an intermediate state. Consequently the $\tau_{x}$ level should have the smallest amount of singlet character and therefore the longest lifetime. With this assignment of the $\tau_{x}$ level and the experimental observation that the $\tau_{z}$. level has the shortest lifetime, the rate constants for the three levels are

$$
\begin{aligned}
& k_{x}=2.5 \mathrm{sec}^{-1} \\
& k_{y}=10 \mathrm{sec}^{-1} \\
& k_{z}=167 \mathrm{sec}^{-1}
\end{aligned}
$$

where the rate constants are equal to the inverse of the lifetimes. 
As was shown in section II (see Eq. 39) the change in intensity of the phosphorescence upon saturating the ESR transition between any two of the three triplet levels is given by

$$
\Delta I_{i j} \approx\left(N_{i}-N_{j}\right)\left(K_{j}-K_{i}\right)
$$

where $1, j=x, y, z$ and $N_{i}$ are the steady state populations of the three triplet levels.

Since the ODMR of all three zero field transitions gave an increase in the phosphorescence intensity, it is clear from Eq. 14 that the populations of the triplet levels must be in an order opposite to that of their rate constants (i.e., $\mathrm{N}_{\mathrm{x}}>\mathrm{N}_{\mathrm{y}}>\mathrm{N}_{\mathrm{z}}$ ). Therefore the populations are inverted and we have a necessary but not sufficient condition for stimulated emission. It should be noted that our assignment of the ${ }^{\tau} x$ level as the level with the longest lifetime is only necessary to predict a population inversion between the $\tau_{x}$ and $\tau_{y}$ levels. The assignment of the $\tau_{z}$ level as the level with the shortest lifetime is sufficient to establish that $\mathrm{N}_{z}<\mathrm{N}_{x}, N_{y}$ and therefore a population inversion certainly exists for the $D+E$ and $D-E$ zero field transitions: 


\section{The ${ }^{3} \pi \pi$ State of Paradichlorobenzene}

This section is divided into two parts. In the first, the ODMR spectra of paradichlorobenzene $(\mathrm{pDB})$ are presented and the results interpreted in terms of a spin Hamiltonian incorporating the electron spinspin, chlorine nuclear quadrupole and chlorine hyperfine interactions. In the second part, the phosphorescence microwave double resonance technique is used in investigating the vibrational strusture of the ghosphorescence spectrum of $\mathrm{pDB}$. The vibrational structure of the phosphorescence spectra is assigned by analysis of the rclative ohune in Intensity of the phosphorescence upon saturation of the three zero field transitions. Both the ESR and phosphorescence spectra are shown to be consistent with a distortion of the excited state due to a bending of the C-CI bonds into a trans configuration.

\section{Ihe ODMR Spectra of Paradichlorobenzene}

Investigations of the first excited triplet state nf benzene and substituted benzenes has been the subjeot of considerable Interest. $107-109$ The absoriplun and phosphorescence spectra of the first excited triplet state of $\mathrm{pDB}$ by Castro and Hochstrasser ${ }^{71}$ and by George and Morris 97 have raised several questions?

I) Is the spacial symmetry of the excited triplet state of $\mathrm{pDB} \mathrm{B}_{2 \mathrm{u}}$ as proposed 71 or $\mathrm{B}_{1 \mathrm{u}}$ as is benzene? 98

2) Do the chlorines participate significantly in the excitation? 99

3) Is the molecule distorted in the excited state? ${ }^{71}$ 
4) Is the electric field gradient at the chlorines changed significantly upon excitation to the first triplet state? ODMR in zero field has been shown to be a powerful tool for studying the electron distribution of organic molecules in their first excited triplet state. Analysis of the parameters used in the spin Hamiltonian that satisfactorily accounts for the ODMR spectra of $\mathrm{pDB}$ should supply the information necessary to answer the above questions.

\section{Experimental}

The starting material (Eastman Organic white label) was degassed and zone refined for 100 passes. Single crystals of pDB were easily grown by the Bridgeman technique. The experimental arrangement used in the ESR experiments is essentially the same as that shown in Figure 15. The sample was excited with the $2800 \AA$ or $3100 \AA$ region of the mercury arc lamp by use of the appropriate interference filter.

The majority of the ESR spectra were obtained by square wave amplitude modulation of the microwave oscillator with a frequency of 10 to $20 \mathrm{~Hz}$ and a modulation depth of $\geq 25 \mathrm{db}$. The Hewlett Packard microwave sweep oscillator was modified by replacing the timing capacitor used for the range with the fastest sweep time with an assembly which permitted switching between external capacitors. With this arrangement sweep times as long as several thousand seconds were possible, permitting the use of sweep rates as low as $.025 \mathrm{MHz} / \mathrm{sec}$.

Frequency modulation of the microwave field was also employed in the course of this work. This was achieved by combining the sine wave output of the lock-in amplifier and the ramp output of an Exact model 255 function 
generator through the + and - inputs of an operational amplifier. The output of the operational amplifier consisted of a positive ramp voltage with a superimposed sine wave. The magnitudes of the ramp and sine wave voltages were independently adjusted to give the desired sweep width and modulation excursion. The modulation rate (i.e., the frequency of the sine wave) used was varied between 10 and $20 \mathrm{~Hz}$. 'The time constant of the lock in amplifier was varied from 0.3 to 30 seconds depending on the swetp rale of the microwave oscillator.

In addition, experiments were performed without modulation of the microwave field in which case the absolute change in intensity of the phosphorescence was monitored. The effective time constant of the electrometer was varied by changing the external load resistor.

The experimental arrangement used in performing the ENDOR experiments is shown in Figure 17 and the sample arrangement in Figure 18. The Hewlett Packard RF sweep oscillator was also modified to permit sweep times as long as 2000 seconds by use of an external timing capacitor.

The measurement of the ${ }^{35} \mathrm{Cl}$ pure nuclear quadrupole resonance of the ground state of $\mathrm{pDB}$ at $4.2^{\circ} \mathrm{K}$ was achieved with the use of a marginal oscillator described by Fayer and Harris. 100 The sample coil was extended by placing the leads to the coil inside a section of stainless steel tubing hent at a right angle in order to support the coil in a liquid helium dewar. The dewar used in this experiment was constructed by inserting a narrow mouth commercial dewar inside a larger wide mouth dewar. The outer dewar was filled with liquid nitrogen and the inner dewar with liquid helium. This arrangement held helium for about 30 minutes with the sample in place. 
Zeeman modulation was achieved by a selenoid wound around the outer dewar.

The ODMR spectra in zero fleld

The ODMR spectra of $\mathrm{pDB}$ in zero magnetic field have been observed while monitoring the emission from two distinct traps. However, only the ODMR spectra due to the shallow (or $\mathrm{x}$ ) trap will be discussed. The chlorine nuclear quadrupole and hyperfine structure of the ODMR spectra observed for the deep (or y) trap were the same as those for the $\mathrm{x}$ trap; however, the zero field values were about $5 \%$ lower.

The observed ODMR spectra of $\mathrm{pDB}$ are due to the interaction of three isotopically distinct molecular species. The relative natural abundances of the ${ }^{35} \mathrm{Cl}$ and ${ }^{37} \mathrm{Cl}$ isotopes are approximately $3 / 4$ and $1 / 4$ respectively. Since there are two chlorine nuclei per molecule, the fractional distribution of the molecular species are

$$
\begin{aligned}
& \text { I } \quad{ }^{35} \mathrm{Cl}-{ }^{35} \mathrm{Cl}-3 / 4 \times 3 / 4-9 / 16 \\
& \text { II } \quad{ }^{35} \mathrm{Cl}-{ }^{37} \mathrm{Cl}=2(3 / 4 \times 1 / 4)=6 / 16 \\
& \text { III } \quad{ }^{37} \mathrm{Cl}-{ }^{37} \mathrm{Cl}=1 / 4 \times 1 / 4=1 / 16
\end{aligned}
$$

The spectra obtained will therefore be considered as the superposition of the ODMR spectra due to each of the three molecular species: The $\tau_{x} \rightarrow \tau_{z}$ (high frequency) transitions observed using amplitude and frequency modulation are shown in Flgures 34 and 35 respectively. The resonance observed for this transition without modulation of the microwave field is shown in Figure 36. The two remaining electron spin 
$-124-\quad$

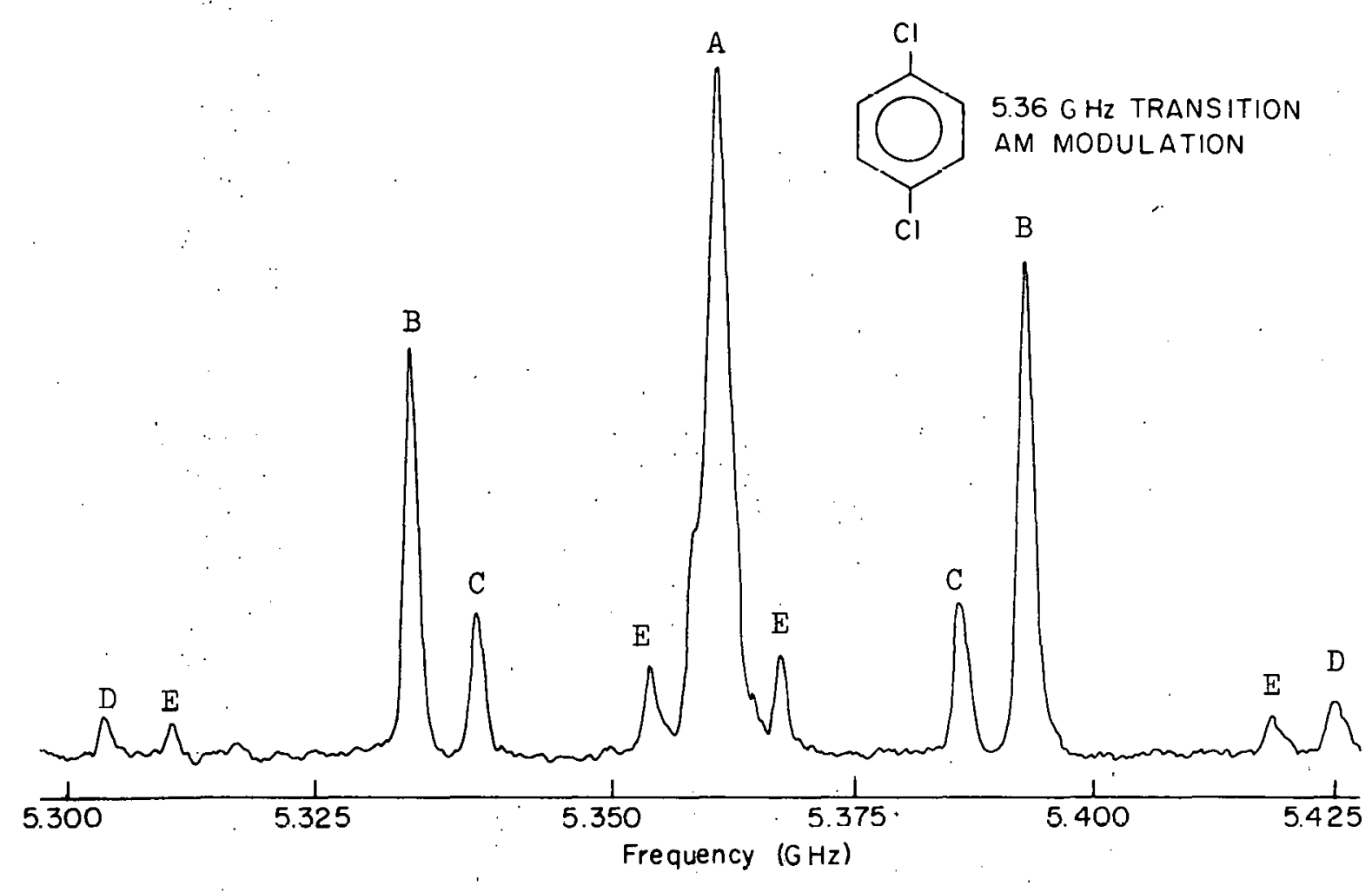

Figure 34

ODMR of the $\tau_{x} \rightarrow \tau_{z}$ multiplet of paradichlorobenzene with amplitude modulation of the microwave field 


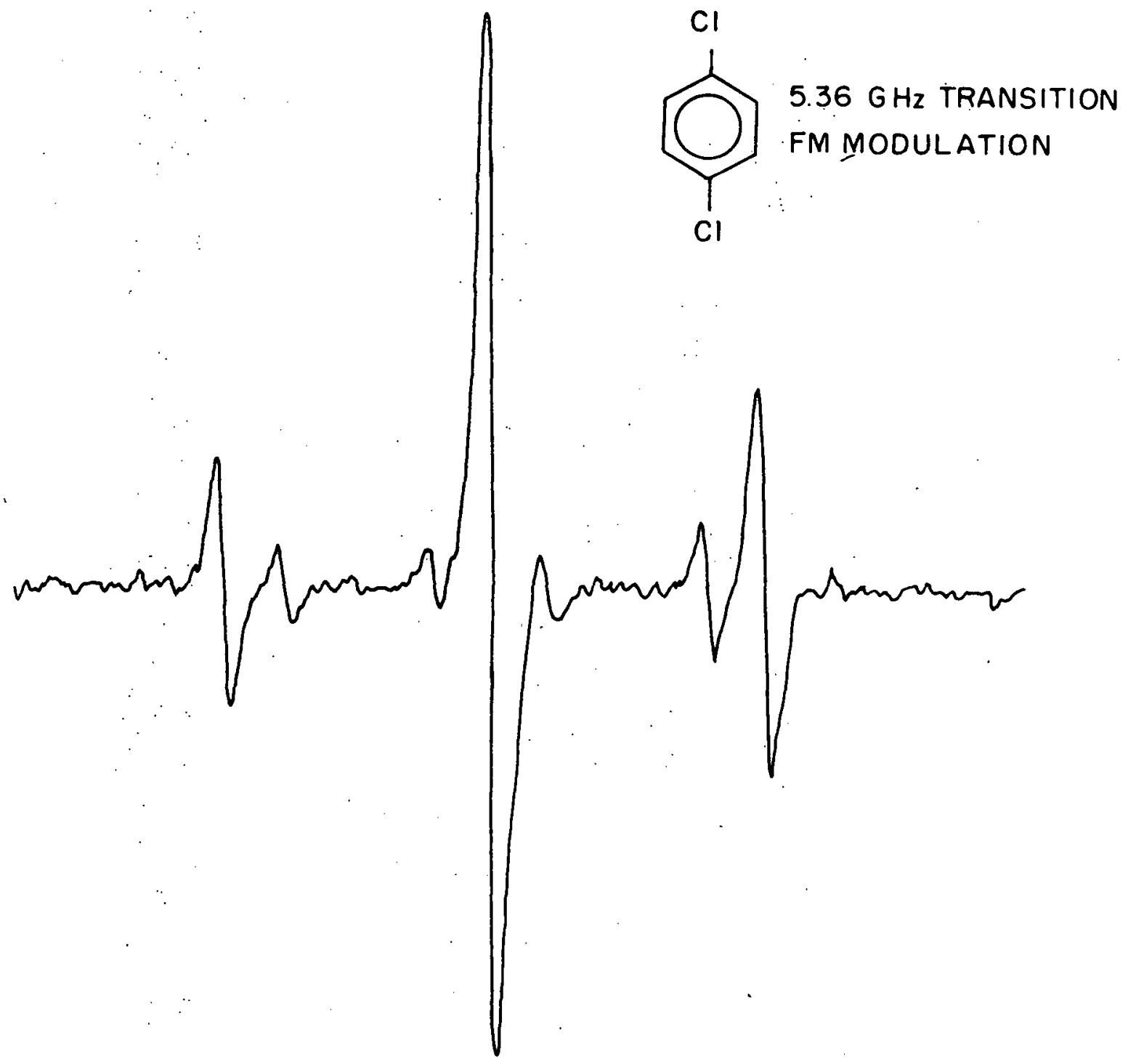

Figure 35

$$
\begin{gathered}
\text { ODMR of the } \tau_{x} \rightarrow \tau_{y} \text { multiplet of paradichlorobenzene with frequency } \\
\text { modulation of the microwave field }
\end{gathered}
$$




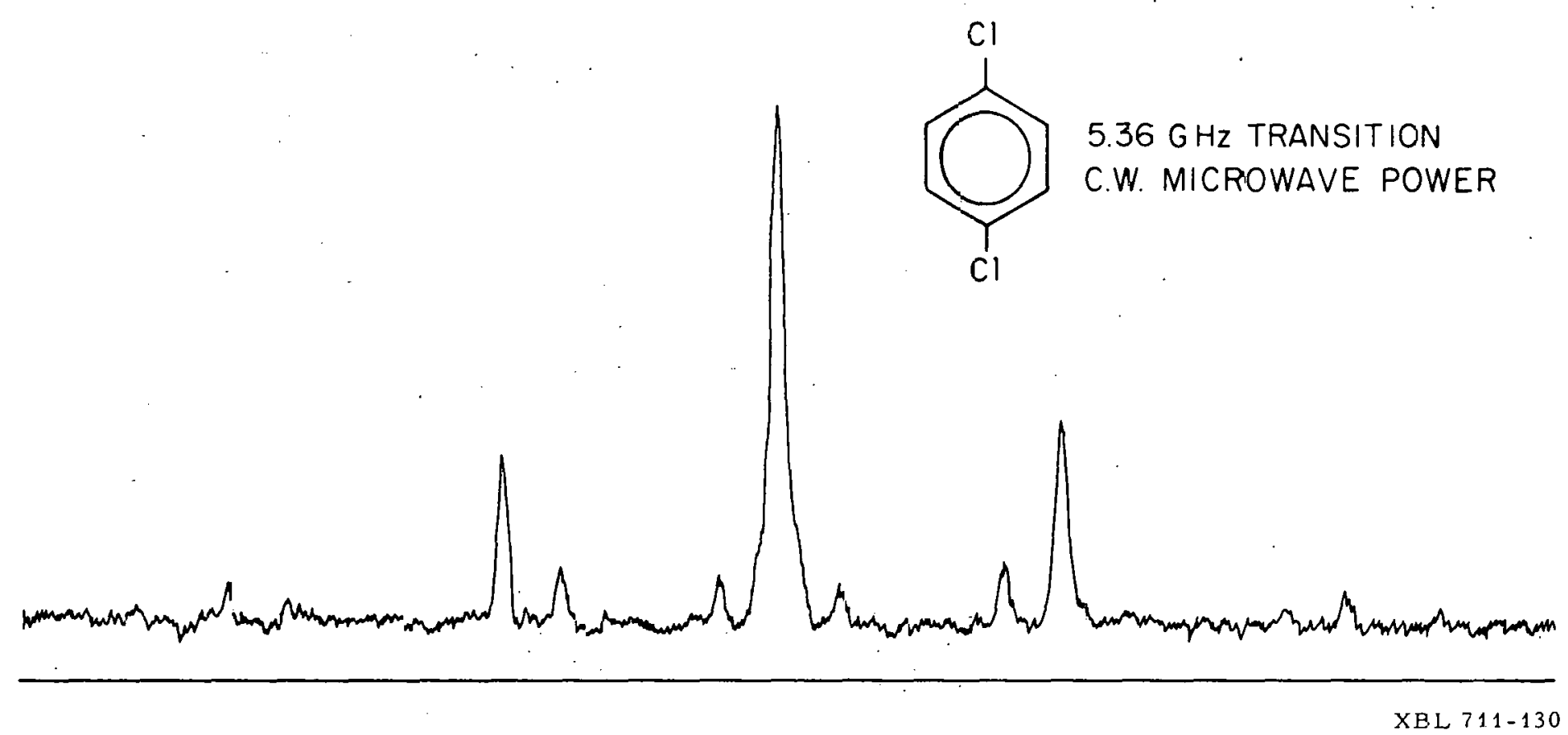

Figure 36

ODMR of the $\tau_{x} \rightarrow \tau_{z}$ nutiplet $0:$ paradichlorobenzene without moculation of the microwave field 
transitions observed using amplitude modulation are shown in Figures $37\left(\tau_{\mathrm{x}} \rightarrow \tau_{\mathrm{y}}\right)$ and $38\left(\tau_{\mathrm{y}} \rightarrow \tau_{\mathrm{z}}\right)$

In table 10 the possible ESR transitions involving the triplet electrons and one or more chlorine nuclei are listed as to type (A, $B$, $C, D, E$, or F) and the possible molecular species (I, II, or III) which can undergo each type of transition. The intensity of the transitions involving the electron and one chlorine spin ( $B$ and $C$ ) and those involving the electron and two chlorine spins ( $D, E$, and $F$ ) must be considered separately. The ratio of the intensities of the transitions involving a single ${ }^{35} \mathrm{Cl}$ spin to those involving a single ${ }^{37} \mathrm{Cl}$ spin is given by

$$
\frac{I_{B}}{I_{C}}=\frac{2(9 / 16)+6 / 16}{6 / 16+2(1 / 16)}=3
$$

Likewise the ratio of the intensities of the transitions involving two chlorine spins is:

$$
I_{D}: I_{E}: I_{F}=9: 6: 1
$$

The structure of the $\tau_{x} \rightarrow \tau_{z}$ electron spin multiplet shown in Figure 34 is labeled according to the classification given in Table 10. Since the nuclear quadrupole moment of ${ }^{35} \mathrm{Cl}$ is larger than that of ${ }^{37} \mathrm{Cl}$, the outer pair of the four strong satellites are assigned as type B $\left({ }^{35} \mathrm{Cl}\right)$ and the inner pair as type $\mathrm{C}$ transitions $\left({ }^{37} \mathrm{Cl}\right)$. As can be seen, the ratio of the intensity of the transitions labeled $B$ and $C$ is approximately 3:1 as predicted. The ratio of the outermost satellites in Figure 34 are assigned to simultaneous double chlorine transitions (labeled D and $E$ on the spectra). The intensity of these transitions is also approximately in the predicted ratio of $9: 6$. 


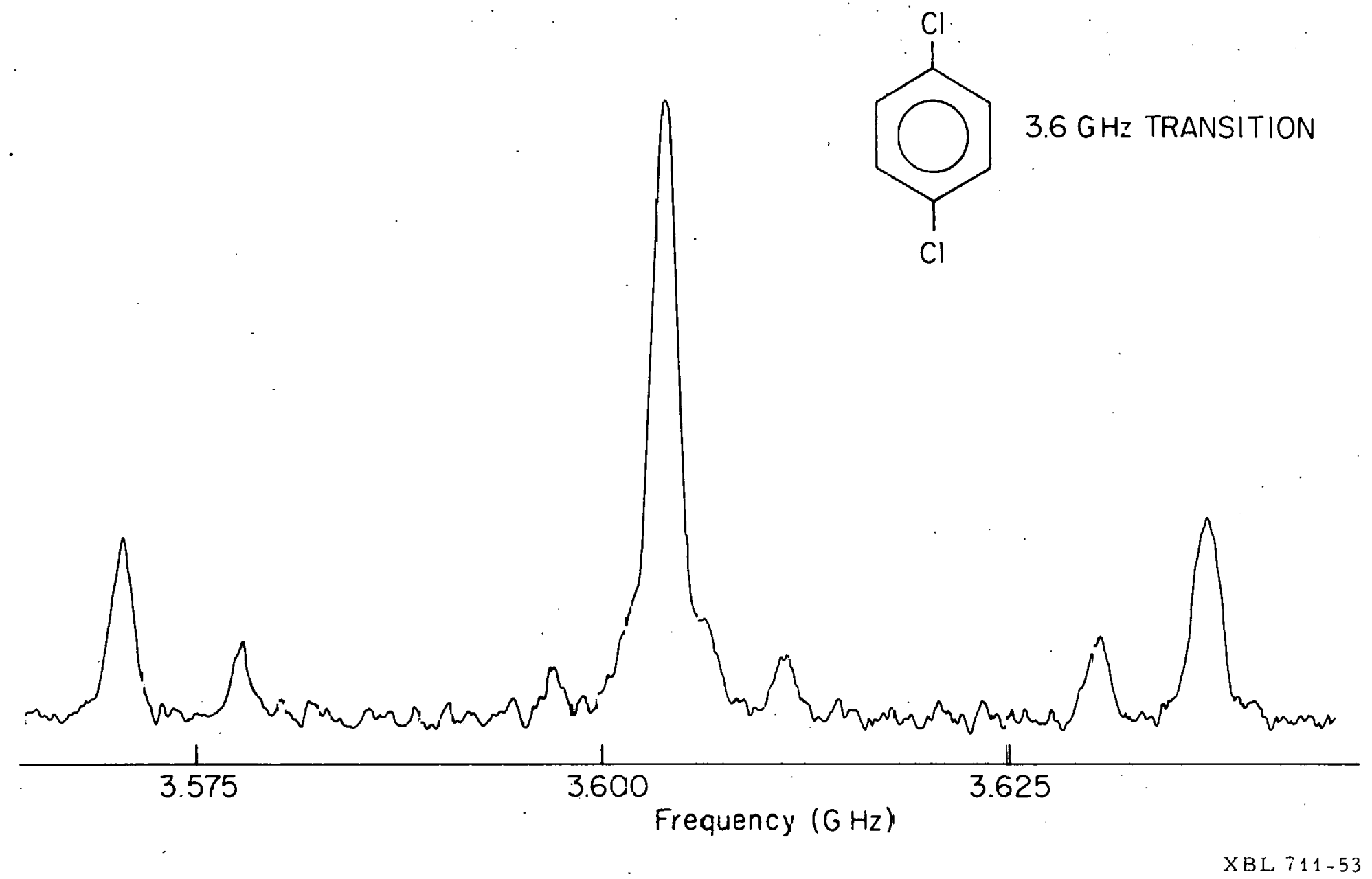

Figure 37

ODMR of the $\tau_{x} \rightarrow \tau_{y}$ multiplet of paradichlorobenzene 


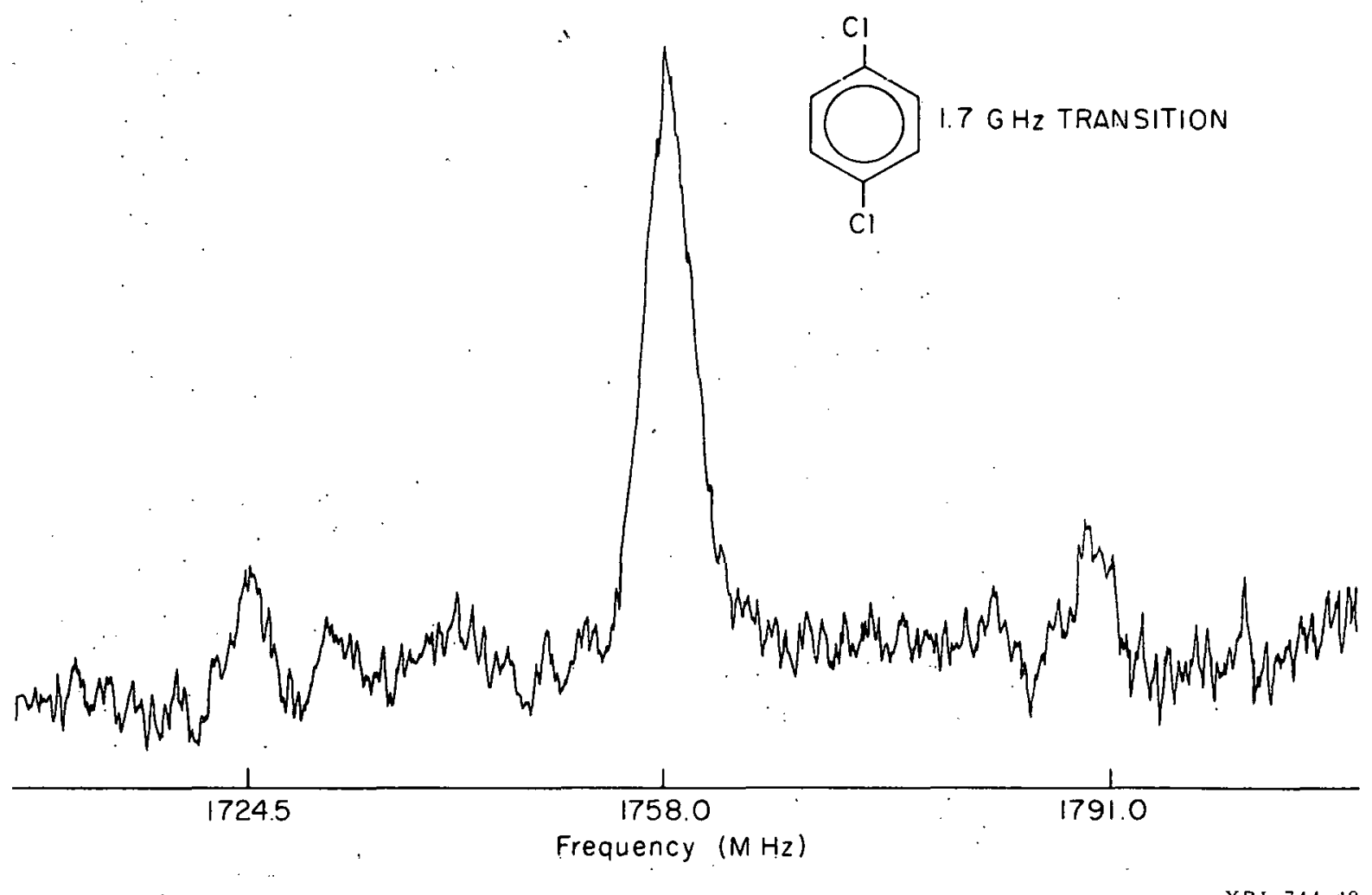

Figure 38

ODMR of the $\tau_{y} \rightarrow \tau_{z}$ multiplet of paradichlorobenzene 


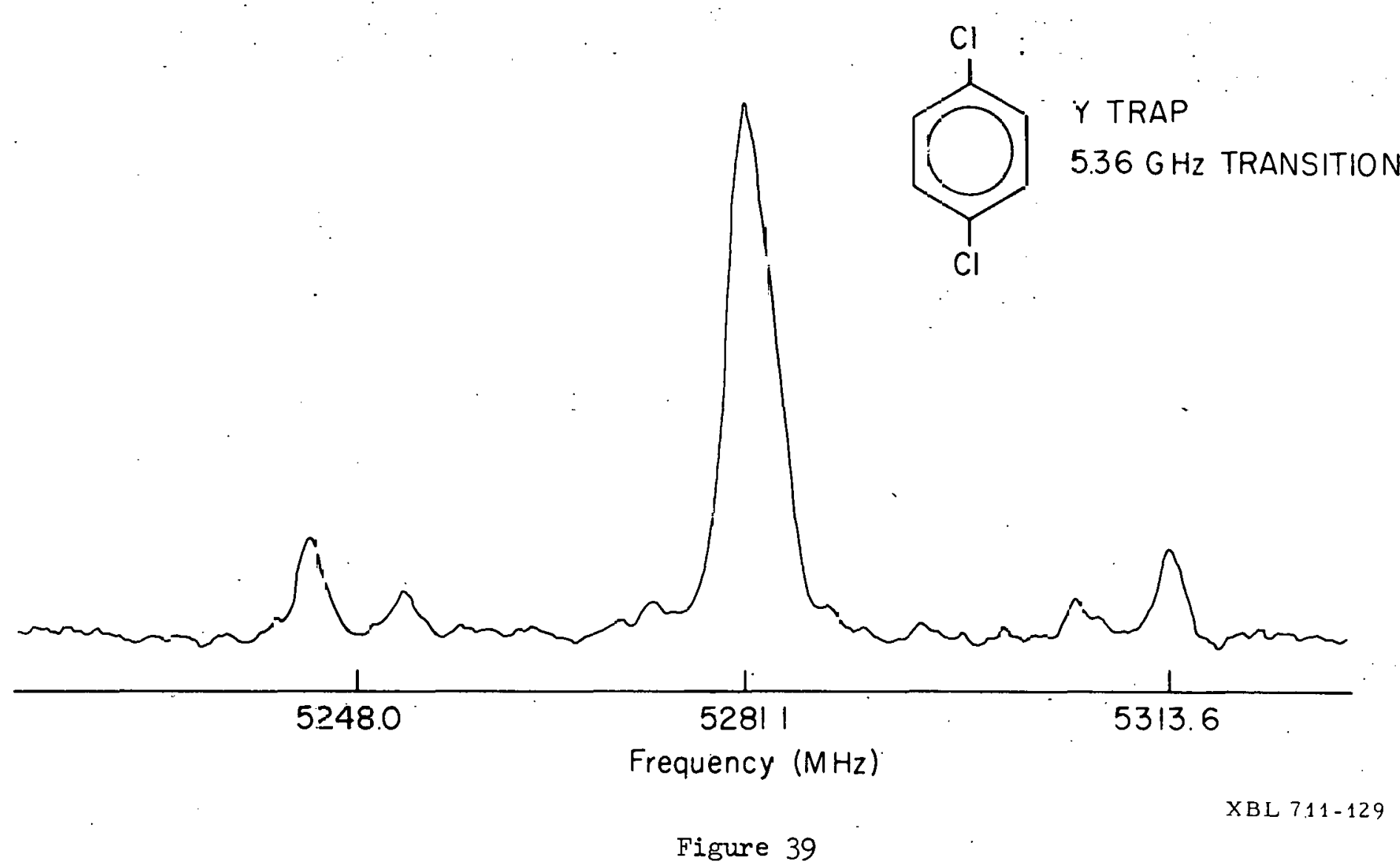

ODMF of the $\tau_{x} \rightarrow \tau_{z}$ multiplet of the $Y$ trap of paracichlorjonzene 
The transitions corresponding to simultaneous double ${ }^{37} \mathrm{Cl}$ transitions (type F) are not observed as would be expected from the small natural abundance of the molecular species responsible for these transitions. The inner pair of satellites (labeled E in Figure 34) may be considered as simultaneous ${ }^{35} \mathrm{Cl}$ and ${ }^{37} \mathrm{Cl}$ transitions, the higher frequency transition representing a ${ }^{35} \mathrm{Cl}$ flip up and a ${ }^{37} \mathrm{Cl}$ flip down, while the lower frequency transition represents a ${ }^{37} \mathrm{Cl}$ flip up and a ${ }^{35} \mathrm{Cl}$ flip down. Since the matrix elements for these double chlorine transitions are significantly different from those associated with the other double chlorine transitions, the intensity of the inner satellites labeled $E$ in Figure 34 should not be compared to the intensity of the outer satellites labeled $D$ and $E$. Chlorine ENDOR transitions were also observed by saturating the ESR transitions in the $\mathrm{D}-\mathrm{E}$ and $\mathrm{D}+\mathrm{E}$ manifold. The ${ }^{35} \mathrm{Cl}$ and ${ }^{37} \mathrm{Cl}$ ENDOR resonances observed by saturating the $D-E$ transition are shown in Figures 40 and 41 respectively, and the ${ }^{35} \mathrm{Cl}$ and ${ }^{37} \mathrm{Cl}$ ENDOR resonances observed by saturating the D + E transition in Figures 42 and 43 respectively.

As an extension of the $\mathrm{Cl}$ ENDOR experiments a ${ }^{35} \mathrm{Cl}$ ENDOR transition was saturated while sweeping the D - E microwave transition. Since only the ENDOR time dependent magnetic field was amplitude modulated and the change in phosphorescnece intensity detected with a lock in amplifier only the ESR transitions that involve a ${ }^{35} \mathrm{Cl}$ spin transition are detected. The spectrumobtained from this experiment is shown in Figure 44 . As can be seen, satellites assigned as simultaneous electron and ${ }^{37} \mathrm{Cl}$ spin transitions (labeled $C$ in Figure 34) are not observed, which confirms the assignment of the chlorine satellites. 


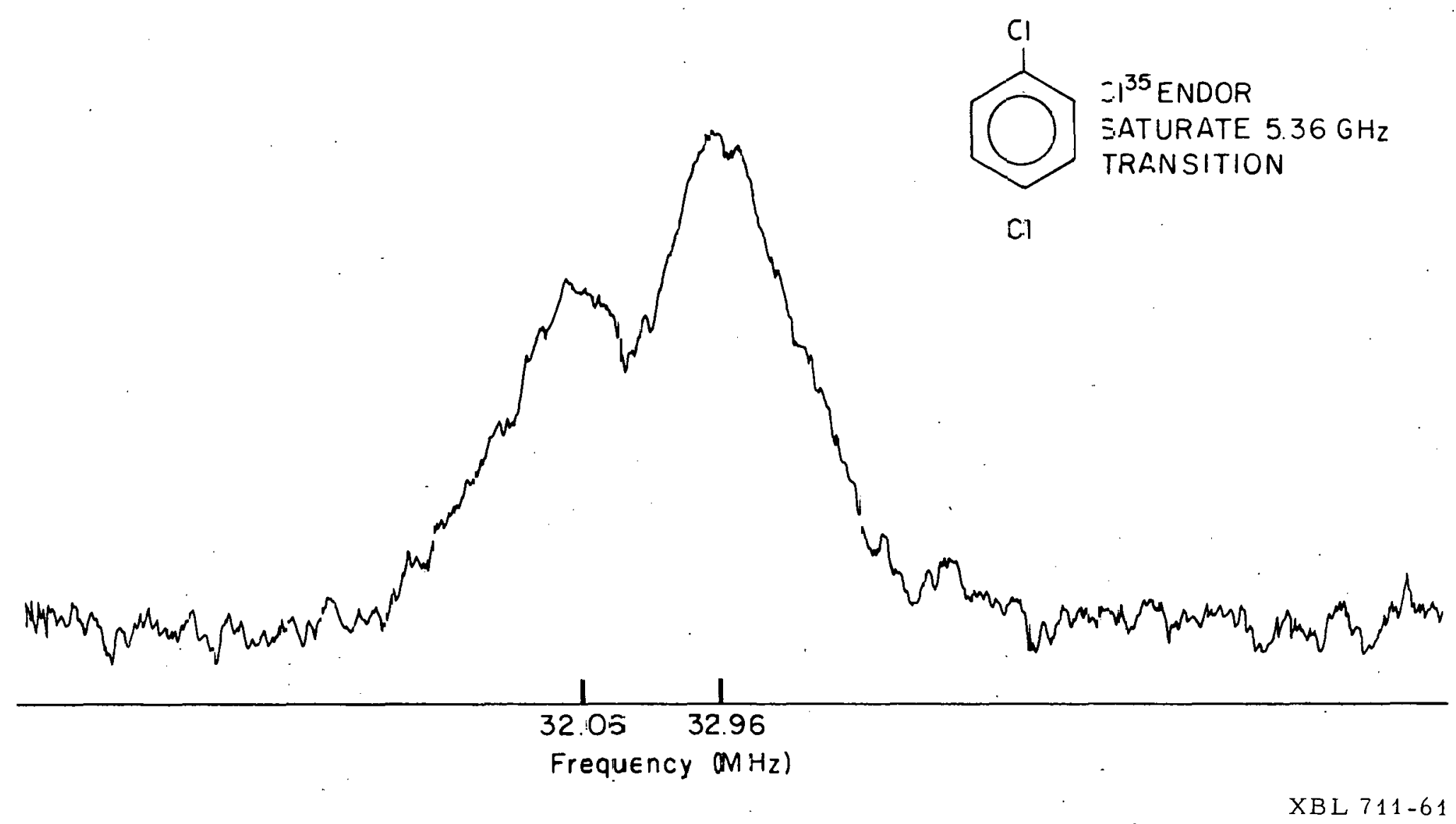

Figdre 40

${ }^{35} \mathrm{Cl}$ ENLOR assoriated with the $\tau_{x} \rightarrow \tau_{z}$ multip_et of paradichlorobenzene 


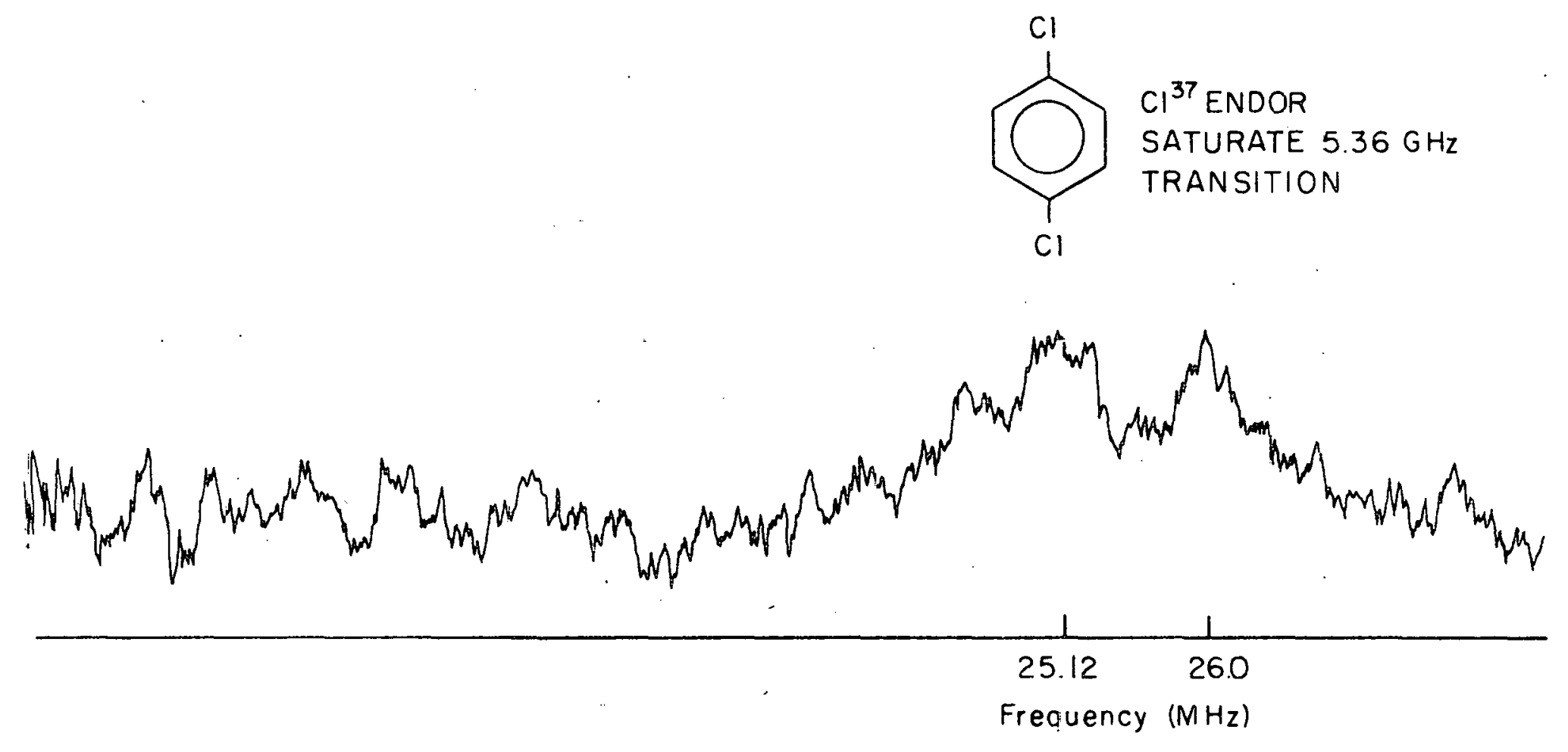

XBL $711-59$

Figure 41

${ }^{37} \mathrm{Cl}$ ENDOR associated with the $\tau_{\mathbf{x}} \rightarrow \tau_{\mathbf{z}}$ multiplet of paradichlorobenzene 


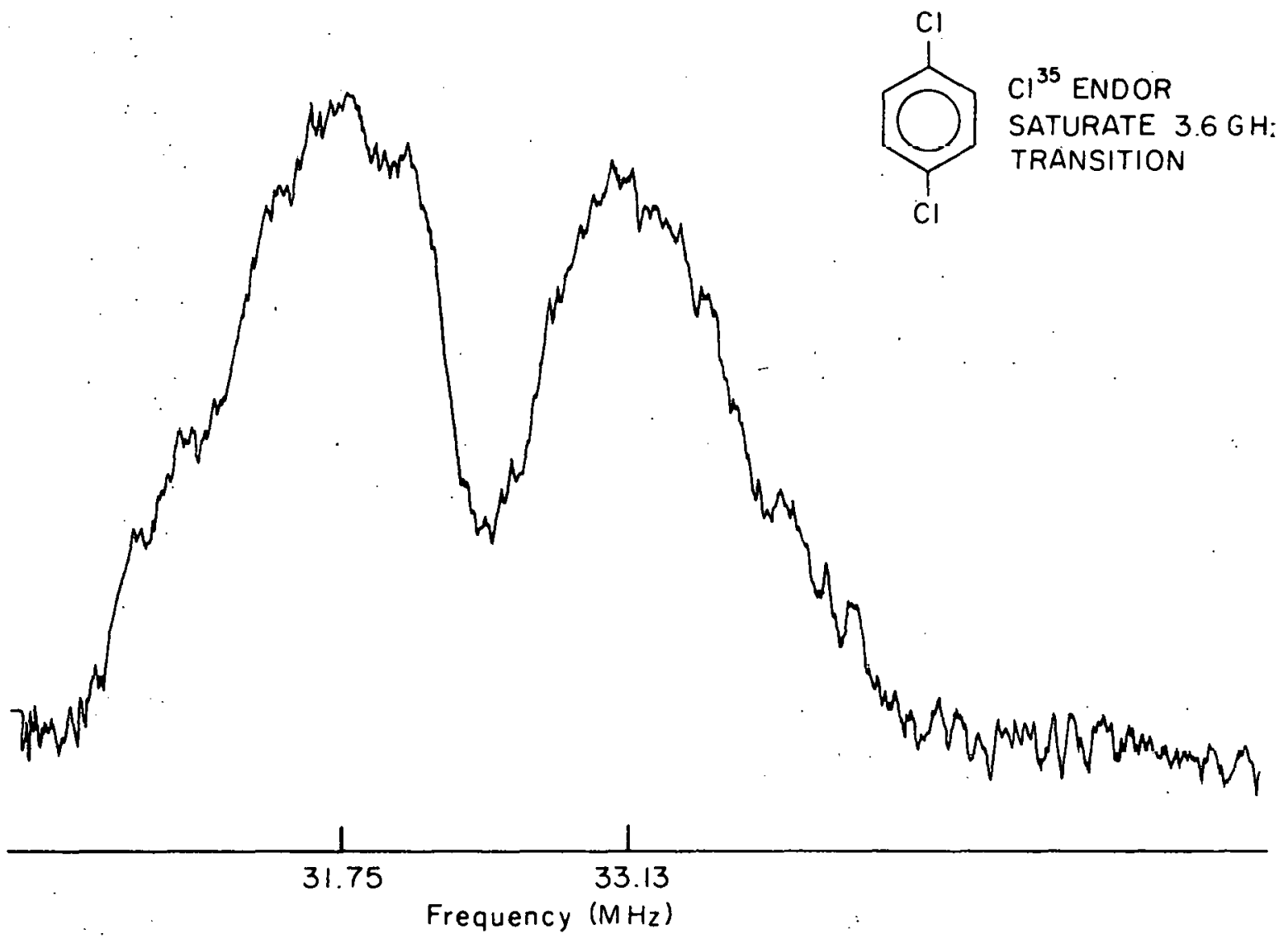

Figure 42

${ }^{35} \mathrm{Cl}$ ENDOR associated with the $\tau_{\mathrm{x}} \rightarrow \tau_{\mathrm{y}}$ multiplet of paradichlorobenzene 


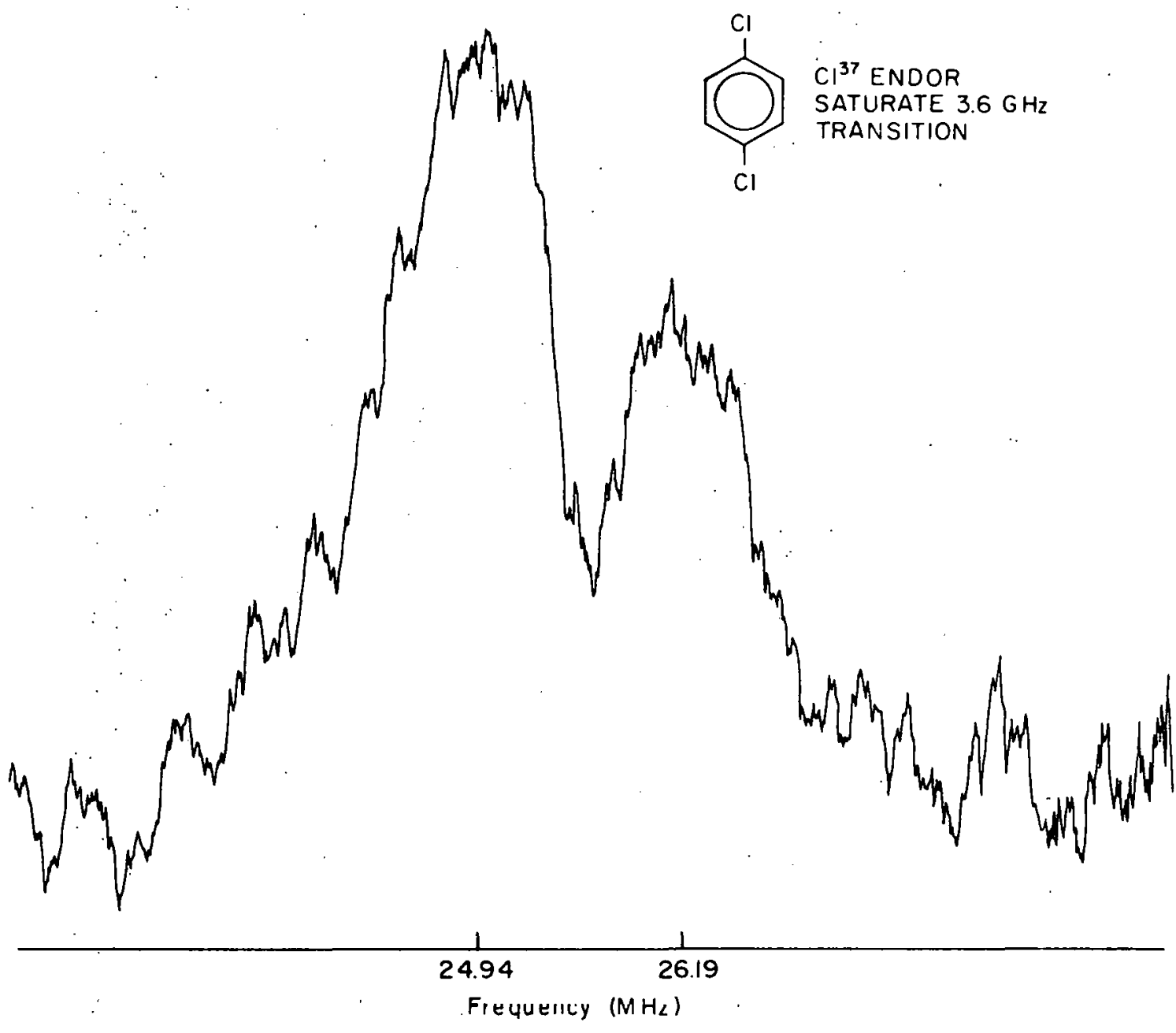

XIIL $711-50$

Figure 43

${ }^{37} \mathrm{Cl}$ ENDOR associated with the $\tau_{\mathrm{x}} \rightarrow \tau_{\mathrm{y}}$ multiplet of paradichlorobenzene 
ENDOR Pumping of Microwave Tronsition

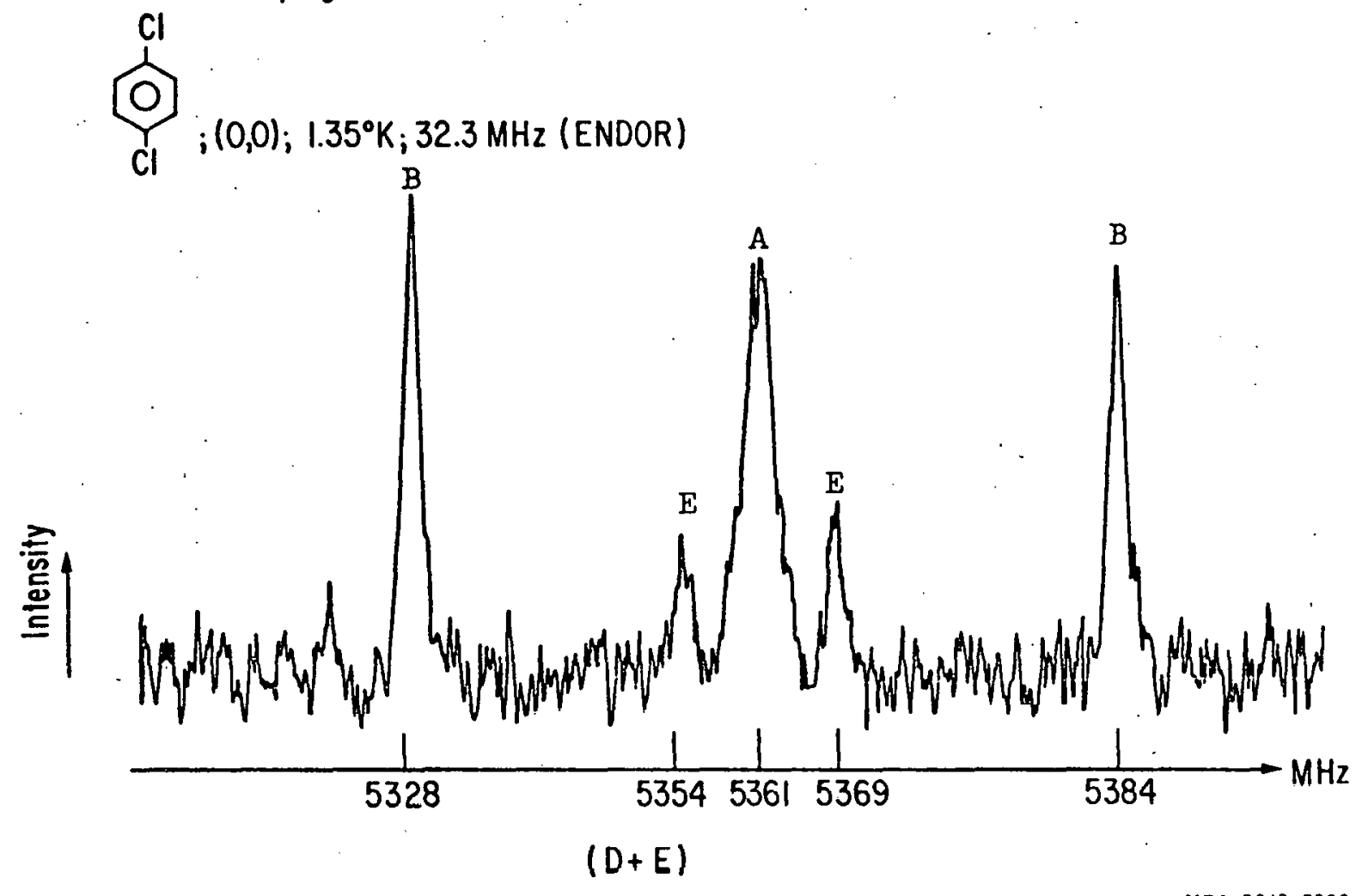

Figure 44

${ }^{35} \mathrm{Cl}$ ENDOR pumping of the $\tau_{x} \rightarrow \tau_{z}$ multiplet in paradichlorobenzene 
The pure nuclear quadrupole resonance spectrum of $\mathrm{pDB}$ in its ground state is shown in Figure 45. Due to the long spin-lattice relaxation time it was difficult to avoid saturation and consequently the signal strength is reduced compared to that obtained at room temperature.

The Spin Hamiltonian

The observed spectra may be satisfactorily explained in terms of a Hamiltonian of the form

$$
\mathrm{H}=\mathrm{H}_{\mathrm{SS}}+\sum_{i} \mathrm{H}_{\mathrm{Q}}+\sum_{i} \mathrm{H}_{\mathrm{HF}}
$$

where the summation is over the chlorine nuclei. With the axis system defined as $x$, out-of-plane; $z$, along the C-Cl bond direction; and $y$, the other in-plane axis, we have,

$$
\begin{aligned}
\mathrm{H}_{\mathrm{SS}} & =-\mathrm{XS}_{\mathrm{x}}{ }^{2}-\mathrm{YS}_{\mathrm{y}}{ }^{2}-\mathrm{ZS}_{\mathrm{z}}{ }^{2} \\
\mathrm{H}_{\mathrm{Q}} & =\frac{\mathrm{e}^{2} \mathrm{qQ}}{12}\left(3 \mathrm{I}_{\mathrm{z}}{ }^{2}-15 / 4\right) \\
\text { and } \quad \mathrm{H}_{\mathrm{HF}} & =\mathrm{A}_{\mathrm{Xx} \mathrm{S}_{\mathrm{X}} \mathrm{I}_{\mathrm{x}}} .
\end{aligned}
$$

The chlorine nuclear quadrupole asymmetry parameter has been assumed to be zero since it is only a small off-diagonal term in the spin Hamiltoni an and could therefore not be determined within the experimental accuracy of our measurements. In addition the chlorine hyperfine interaction was found to be characterized satisfactorily by considering only the out-ofplane hyperfine interaction. Since these experiments were performed in the absence of an external magnetic field, no information is obtained 


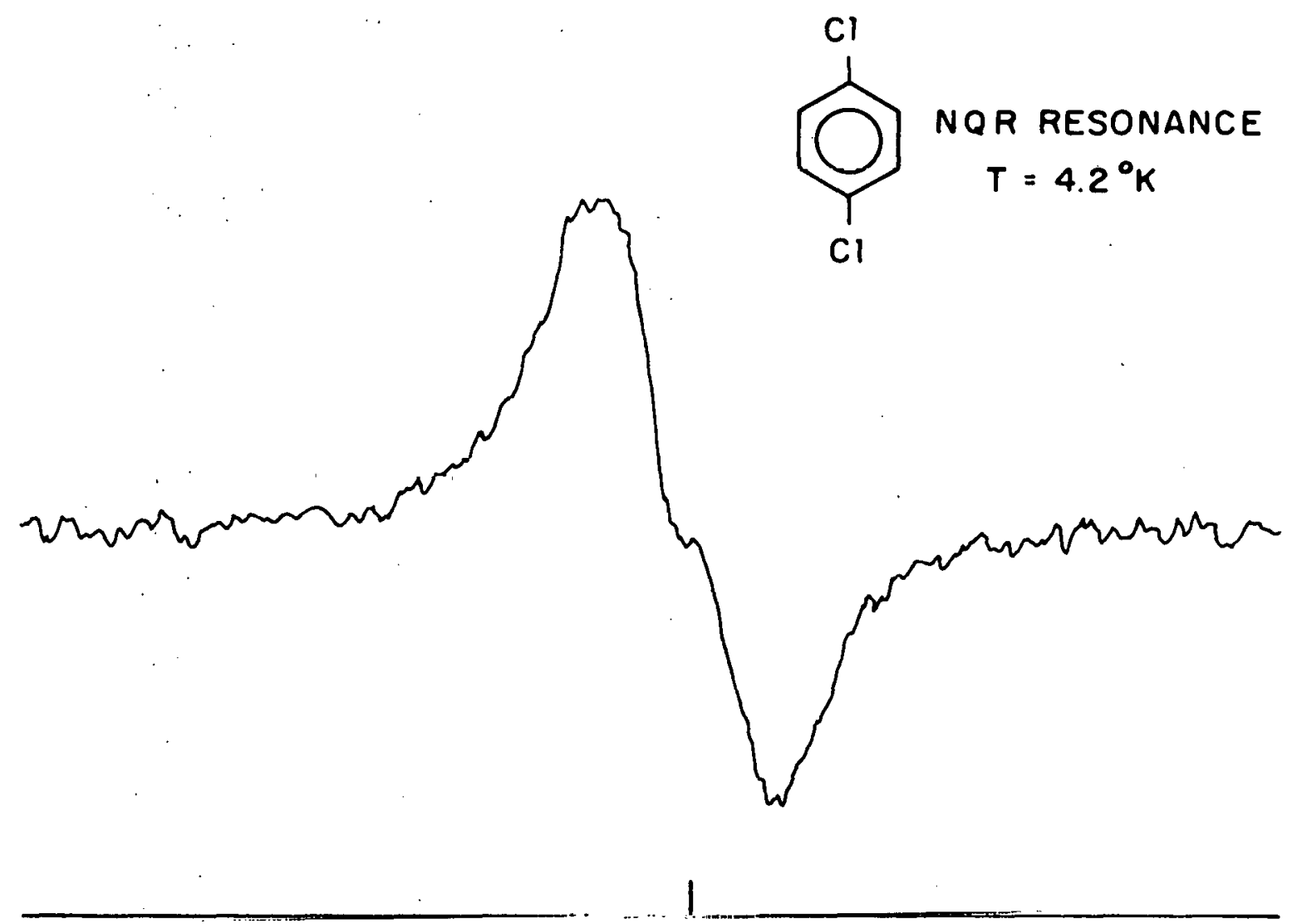

34. 831

Frequency $(\mathrm{MHz})$

XRI. $711-126$

F1gurc 45

Pure NQR resonance of the ground state of paradichlorobenzene 
from the ESR spectra as to the relative orientation of $\mathrm{H}_{S S^{\prime}} \mathrm{H}_{Q}$ and $\mathrm{H}_{H F}$. Therefore; on the basis of single crystal ESR studies of other molecules in $\pi \pi^{*}$ triplet states, 27,42 it was assumed that the axis systems are coincident. In addition, Zeeman studies of the pure nuclear quadrupole resonance of $\mathrm{pDB}$ in its ground state have shown that the chlorine nuclear quadrupole principal axis system is within one degree of the C-Cl bond direction as determined from $x$-ray studies. 110,111 We have also made the assumption that the hyperfine interaction due to the four protons may be neglected. This is justified on the basis that the interaction would cause only a small broadening and shift of the transitions and could not be resolved within our experimental accuracy. Furthermore, no resolveable hyperfine interaction attributable to protons has been reported to date for ODMR experiments with zero external magnetic field.* The basis states used in calculating the spin Hamiltonian and the effect of $\mathrm{H}_{Q}$ and $\mathrm{H}_{\mathrm{HF}}$ on the transition frequencies and intensities have been treated in detail in sections III and $V-A$ and will therefore not be repeated here.

The total spin of the system is 4 and therefore we do not have Kramers degeneracy and must consider the 48 separate energy levels of the excited triplet state. The ODMR spectra were simulated by use of a computer program (see Appendix) that diagonalized the spin Hamiltonian and calculated the transition frequencies and intensities. The spectra were fitted by calculating the spectra fior the ${ }^{35} \mathrm{Cl}-{ }^{35} \mathrm{Cl}$ species of $\mathrm{pDB}$. After the best fit to these transitions was obtained, the nuclear quadrupole coupling constant of ${ }^{35} \mathrm{Cl}$ was multiplied by the ratio of the ${ }^{37} \mathrm{Cl}$ nuclear quadrupole * However, Hutchison and his group have observed the proton hyperfine interaction in the ${ }^{3} \pi \pi^{*}$ state of naphthalene using conventional ESR techniques. 124 
moment to the ${ }^{35} \mathrm{Cl}$ nuclear quadrupole moment $(0.78815)^{112}$ in order to obtain the ${ }^{37} \mathrm{Cl}$ nuclear quadrupole coupling constant. The ${ }^{37} \mathrm{Cl}$ hyperfine interaction was obtained in a similar fashion by multiplying the ${ }^{35} \mathrm{Cl}$ hyperfine interaction $\left(A_{x x}\right)$ by the ratio of $r_{37} \mathrm{Cl}$ to $r_{35} \mathrm{Cl}(0.8322)$. The elements of $\mathrm{H}_{\mathrm{SS}}$ used in simulating the spectra observed while monitorIng the $x$ trap emission are listed in Table 11 along with the approximate values of $\mathrm{H}_{\mathrm{SS}}$ for the $\mathrm{y}$ trap and the values reported for benzene. 93 The best value of the ${ }^{35} \mathrm{Cl}$ nuclear quadmpole compling sonstant was -64.50 $\mathrm{MHz}\left({ }^{37} \mathrm{Cl}=-50.84 \mathrm{MHz}\right)$ and for the ${ }^{35} \mathrm{Cl}$ hyperfine interaction $\mathrm{A}_{\mathrm{xx}}=22$ $\mathrm{MHz}\left({ }^{37} \mathrm{Cl} ; \mathrm{A}_{\mathrm{xx}}=18.3 \mathrm{MHz}\right)$. The experimental and calculated ESR frequencico for the $x$ trap of $\mathrm{pDB}$ are listed in Table 12. With the parameters used in the spin Hamiltonian all of the calculated transition frequencies are within experimental error. In order to observe the simultaneous chlorine and electron spin transitions it was necessary to use a large microwave field (approximately one watt). Since there are several transitions iiffering slightly in frequency that correspond to a particular type of transition ( $A, B, C$, etc.), assigning one average frequency to a transition is dift'icult since the magnitude of the time dependent magnetis field and the relaxation rate constants are not known. Therefore a simple weighted average of the transitions corresponding to a particular type was made which introduces a small error in the calculated frequencies.

The observed and calculated chlorine ENDOR transitions are listed in Table 13. The ENDOR resonances associated with the D - E multiplct are due to transitions in the $x$ and $z$ triplet levels whereas those observed while saturating the $D+E$ multiplet are due to transitions in 
the $\mathrm{y}$ and $\mathrm{z}$ triplet levels. In addition to the linewidth of the ENDOR transitions the large time dependent magnetic fields used in the ENDOR experiments (approximately twenty watts) makes the proper weighting of the calculated ENDOR frequencies unknown. Therefore only the range of calculated ENDOR frequencies is listed in Table 13.

\section{Discussion}

From previous experimental 11,94 and theoretical $113-116$ studies of aromatic molecules in $\pi^{*}$ triplet states it is almost certain that in pDB the largest component of the electron spin-spin tensor in its principal axis system is along the molecular axis normal to the plane (x). Indeed this is what is observed for the $\pi^{*}$ triplet state of benzene. 93 The ordering of the interaction along the two in-plane molecular axes is not immediately apparent, From the analysis of the PMDR spectra of pDB (which is treated in the following section) the component of the electron spin-spin interaction along the molecular $z$ (or long in-plane axis) is assigned as the larger of the two in-plane components of the hyperfine tensor.

Since the zero field splitting parameters $D$ and $D^{*}\left(D^{*}=\left(D^{2}+3 E^{2}\right)^{\frac{1}{2}}\right)$ are primarily a function of the size of the $\pi$ system involved in the excitation, 117 the value of these parameters for both $\mathrm{pDB}$ and benzene should be similar if $\mathrm{pDB}$ is a $\pi^{*}$ triplet. As can be seen in Table 11 the values of $D$ and $D^{*}$ for both traps of $\mathrm{pDB}$ differ from the corresponding values for benzene by only a few percent which confirms the assignment of the excited triplet state of $\mathrm{pDB}$ as a $\pi^{*}$ state. 
The zero field splitting parameter $E$ which is a measure of the anisotropy of the triplet electron distribution in the molecular plane is however quite different for both molecules. If the benzene molecule possessed $D_{6 h}$ symmetry in the excited state, $E$ must be zero by symetry. The finite value of $E$ for benzene has been explained by deGroot and van der Waals ${ }^{101}$ on the basis of a distortion of the benzene ring from the symmetric $D_{6 h}$ benzene with bond lengths of $1.427 \AA$ to a compressed $D_{2 h}$ benzene with four long bonds $(1.448 \AA$ ) and two short bonds (1.381 $\AA$ ). Godfrey, Kern, and Karplus ${ }^{102}$ have evaluated the integrals exactly and have explained the observed zero field splitting by use of a symmetrical structure for the benzene molecule and adjusting the extent of configurational mixing. They also calculated the effect of adding a substituent along our $z$ axis with 2 electrons in a $\Gamma_{x}$ orbital und found that if the benzene configurations are assumed to maintain the same relative weights as undistorted benzene and if the small matrix elements between the charge transfer and the benzene configurations are neglected, the values of $D$, $E$ and $D^{*}$ : decrease linearly in magnitude with increasing charge transfer. As will be discussed in the next section,

$t$ he vilue of the zero field splitting parameter $E$ for $\mathrm{pDB}$ is of the opposite sign as that predicted by Godfrey, Kern and Karplus. 102 The largest in-plane zero field axis in pDB is along the molecular $z$ axis while the theoretical studies predict the largest in-plane zero field axis along the molecular $y$ axis.

The discrepancy between the zero field splitting of $\mathrm{pDB}$ and that predicted on the basis of treating the chlorines as a small perturbation of the excited state of benzene raises the possibility that the symetry 
of the excited state of $\mathrm{pDB}$ is different than that of the excited state of benzene. In any case the zero field tensor of $\mathrm{pDB}$ is consistent with a triplet spin distribution in which the spin density on the two carbon atoms to which the chlorines are bonded is much smaller than the spin density on the other four carbon atoms.

The value of the chlorine nuclear quadrupole coupling constant $\left(e^{2} q Q\right)$ In the excited state of $\mathrm{pDB}$ is significantly reduced compared to the corresponding value for the ground state.

With the assumption that the asymmetry parameter $\eta$ may be neglected, the value of $e^{2} q Q$ for the ${ }^{35} \mathrm{Cl}$ nuclei of $\mathrm{pDB}$ in its excited triplet state at $1.3^{\circ} \mathrm{K}$ is $-64.5 \mathrm{MHz}$. The measured pure nuclear quadrupole resonance frequency of $\mathrm{pDB}$ in its ground state at $4.2^{\circ} \mathrm{K}$ is $34.83 \mathrm{MHz}$ which, if $\eta$ is assumed to equal zero, corresponds to a value of $e^{2} q Q$ of $-69.662 \mathrm{MHz}$. The assumption that $\eta$ may be neglected is justified on the basis that $e^{2} q Q$ is not changed significantly for small value of $\eta$ and for the ground state of $\mathrm{pDB}$ at room temperature $\eta$ is on $7 \mathrm{y} 0.08 .^{108}$ Referring to Figure 7 , it may be seen that the assumption that the pure NQR transition frequency $v$ equals $\left|\frac{1}{2} e^{2} q Q\right|$ causes a positive error of less than $5 \%$ for $\eta \leq 0.5$. The sign of $e^{2} q Q \cdot i s$ not obtained from either the measurement of the pure NQR transition. frequency or the spin Hamiltonian; however, from other theoretical and experimental studies $e^{2} q Q$ for $C l$ is known to be negative for covalently bonded compounds.

The increase of $52 \mathrm{KHz}$ in the pure $\mathrm{NQR}$ frequency of the ground state of pDB upon lowering the temperature of the sample from $77^{\circ} \mathrm{K}(v=34.779$ $\mathrm{MHz})$ to $4.2^{\circ} \mathrm{K}(v=34.831 \mathrm{MHz})$ is consistent with Bayer's theory $11^{8}$ 
which treats the temperature dependence of the NQR frequency in terms of the molecular torsional motions. The small change in the pure NQR transition frequency indicates that there is no major physical change in the environment of the chlorine nuclei in $\mathrm{pDB}$ upon cooling. Therefore the difference in $\mathrm{e}^{2} \mathrm{qQ}$ between the ground and excited states of $\mathrm{pDB}$ is clearly due to a change in the electric field gradient $(q)$ at the chlorines urm excitation. In contrast to the electron spin-spin and hyperfine interactions which are a function of the triplet electrons only, $e^{2} q Q$ is independent of the spin of the electrons.

Since electrons in $s$ orbitals have spherical symetry, they do not contribute to the field gradient. A closed $\mathrm{p}$ shell also contributes nothing to the field gradient, and therefore following the analysis of Bersohn 103 the field gradient in pDB con bc considered as arlsing from a hole in the $p_{z}$ orbital and a partial hole in the $p_{x}$ orbital. The total contribution is due to two axially symetric tensors whose major axes are perpendicular. In Table 14, the contributions to the field gradients are expressed in terms of the number of holes in the $\mathrm{p}_{x}$ and $\mathrm{p}_{z_{\mathrm{r}}}$ chlorine orbitals.

The difference in $\mathrm{e}^{2} \mathrm{qQ}$ for the excited and ground state may be written,

$$
\Delta e^{2} q Q=e^{2}\left(q_{T}-q_{G}\right) Q
$$

where $q_{T}$ and $q_{G}$ refer to the field gradient at the chlorines in the triplel and ground states of $\mathrm{pDB}$ respectively. Equation 6 may be expressed in terms of the number of holes in the $p_{z}$ and $p_{x}$ orbitals as

$$
\Delta \mathrm{e}^{2} \mathrm{qQ}=\mathrm{e}\left[\left(\sigma_{\mathrm{T}}-\sigma_{\mathrm{G}}\right)-\frac{1}{2}\left(\delta_{\mathrm{T}}-\delta_{\mathrm{G}}\right)\right] \mathrm{Q}
$$


Since $\Delta e^{2} q Q$ is negative, one of the following three conditions must be met, a) $\sigma_{G}>\sigma_{\mathrm{T}}$, b) $\delta_{\mathrm{T}}>\delta_{\mathrm{G}}$, or c) $\sigma_{\mathrm{G}}>\sigma_{\mathrm{T}}$ and $\delta_{\mathrm{T}}>\delta_{\mathrm{G}^{*}}$. If $\sigma_{G}$ is greater than $\sigma_{T}$, the number of holes has decreased along the carbonchlorine bond, and therefore the chlorine nuclei are more successful in competing for electrons in the excited state. However since the sigma electrons are not involved in the excitation,this effect should be very small.

If $\delta_{T}$ is greater than $\delta_{G}$, the out-of-plane chlorine porbital has lost electrons. An increase in the number of holes in the $\mathrm{p}_{\mathrm{x}}$ orbital would be the most likely explanation of the decrease in $e^{2} q \dot{q}$ since the chlorine $\mathrm{p}_{\mathrm{x}}$ orbitals are allowed by symetry to interact with the carbon $\mathrm{p}_{\mathrm{x}}$ orbitals. The increase in the number of holes in the chlorine $\mathrm{p}_{\mathrm{x}}$ orbital can come about from either an increase in the double bond character of the $\mathrm{C}-\mathrm{Cl}$ bond or a "bent" $\mathrm{C}-\mathrm{Cl}$ bond. Bray, Barnes and Bersohn 104 have shown that although the overlap of the carbon and chlorine $p_{x}$ orbitals is reduced with a bent $\mathrm{C}-\mathrm{Cl}$ bond, the chlorine $\mathrm{p}_{\mathrm{x}}$ orbitals may overlap with the carbon sigma system, consequently increasing the number of holes in the $\mathrm{p}_{\mathrm{x}}$ orbital of chlorine $\left(\delta_{\mathrm{T}}\right)$ relative to the number of holes in the $\mathrm{p}_{\mathrm{x}}$ orbital. in the ground state $\left(\delta_{G}\right)$. From the analysis of the zero field tensor it appears that the triplet electrons do not have a large spin density on the carbon atoms bonded to the chlorines. Therefore an appreciable increase in $\pi$ bonding seems unlikely. This leaves us with the possibility of a bent $\mathrm{C}-\mathrm{Cl}$ bond. This would require a small value for the chlorine hyperfine interaction since the $\mathrm{C}-\mathrm{Cl} \mathrm{p}_{\mathrm{x}}$ overlap is reduced. The interpretation of the observed chlorine hyperfine interaction is limited since only one of the three components of the hyperfine tensor 
was measured. The magnitude of the chlorine hyperfine interaction is a function of both the spin density on the chlorine and the ring carbon to which it is bonded. ${ }^{105,106}$ However, we will limit our discussion to a simple analysis in which only the spin density on the chlorine is considered to contribute to the hyperfine interaction. The theoretical hyperfine interaction due to one unpaired electron in a carbon $P$ orbital is $280 \mathrm{MHz}$. 89 Neglecting any isotropic contribution to the measured hyperfine interaction, the spin density in each shlorine $p_{x}$ orbital io,

$$
\rho \approx 22 / 280=.08
$$

where $\rho$ is normalized to two. Although this is a very crude approximation, it is clear that the chlorines do not participate significantly in the excltation. This small value for $\rho$ is also consistent. with a bent $\mathrm{C}-\mathrm{Cl}$ bond rather than an increased double bond character since in the latter case a significantly larger value of $\rho$ would be expected. Since all three of the parameters obtained from the spin Hamiltonian support the hypothesis that the $\mathrm{C}-\mathrm{Cl}$ bond is bent, it seems the most reasonable conclusion. The molecule in the excited statc may therefore exist in a cis $\left(C_{2 v}\right)$ or trans $\left(D_{2 h}\right)$ configuration. In order to resolve this question; the phosphorescence.spectra have been analyzed by investigating the relative changes in the vibronic structure of the phosphorescence upon inducing ESR transitions between the three triplet sublevels. The result of this analysis is given in the following section. 


$$
-147-
$$

Table 10

ESR Transitions in Paradichlorobenzene

Transition

Type

Simultaneous Transitions Molecular Species

A Electron Spin . I, II, III

B Electron and ${ }^{35} \mathrm{Cl}$ spins I, II

C Electron and ${ }^{37} \mathrm{CI}$ Spins II, III

D. Electron, ${ }^{35} \mathrm{Cl}$ and ${ }^{35} \mathrm{Cl}$ spins I

E Electron, ${ }^{35} \mathrm{Cl}$ and ${ }^{37} \mathrm{Cl}$ spins II

F Electron, ${ }^{37} \mathrm{Cl}$ and ${ }^{37} \mathrm{Cl}$ Spins III 
Table 11

Zero Field Splitting Parameters (MHz)

\begin{tabular}{|c|c|c|c|}
\hline & \multicolumn{2}{|c|}{ Paradichlorobenzene } & $\begin{array}{c}\text { Benezene-h/ } \\
\text { In Benzene-d } 6\left(1.95^{\circ} \mathrm{K}\right)\end{array}$ \\
\hline$X$ & -2988.75 & $-2967 \cdot 7$ & -3159.8 \\
\hline$Y$ & 616.07 & 654.4 & 1769.4 \\
\hline 乙 & 2372.68 & 2313.4 & 138.5 .0 \\
\hline$D^{\ddagger}$ & $4483 \cdot 13$ & 4451.6 & 4739.7 \\
\hline$E^{\neq}$ & 878.31 & 829.5 & -192.2 \\
\hline$D^{*}$ & 4733.8 & $4677 \cdot 7$ & 4793.2 \\
\hline \multicolumn{4}{|c|}{$\begin{array}{l}\text { In order to be consistent with the standard ESR definitions we } \\
\text { have defined }\end{array}$} \\
\hline \multicolumn{4}{|c|}{$D=-3 / 2 X$ and $E=I / 2(Z-Y)$} \\
\hline
\end{tabular}


Table 12

Measured and'Calculated ESR Transitions of the ${ }^{3}{ }_{\pi \pi}^{*}$ State of Paradichlorobenzene (X Trap)

\section{Measured Frequency Classification}

a) $\tau_{x} \rightarrow \tau_{z}$

$\begin{array}{llll}5426.7 & 1.0^{*} & 5426.91 & \mathrm{D} \\ 5419.6 & 1.0^{*} & 5419.56 & \mathrm{E} \\ 5394.56 & 0.41 & 5394.62 & \mathrm{~B} \\ 5387.86 & 0.41 & 5387.79 \\ 5368.73 & 0.64 & 5368.89 & \mathrm{C} \\ 5362.20 & 0.34 & 5362.14 & \mathrm{E} \\ 5355.13 & 0.25 & 5355.12 & \mathrm{~A} \\ 5336.67 & 0.24 & 5366.50 & \mathrm{E} \\ 5329.74 & 0.28 & 5329.75 & \mathrm{C} \\ 5303.8 & 1.0^{*} & 5304.11 & \mathrm{~B} \\ 5296.5 & .1 .0^{*} & 5297.35 & \mathrm{E}\end{array}$

b) $\tau_{\mathrm{x}} \rightarrow \tau_{\mathrm{y}}$

$$
\begin{aligned}
& 3636.03 \\
& 3629.65 \\
& 3611.18 \\
& 3604.19 \\
& 3597.69 \\
& 3578.90 \\
& 3571.88
\end{aligned}
$$$$
.07
$$$$
3636.13
$$$$
\text { B }
$$$$
.18
$$$$
3629.56
$$$$
\text { C }
$$$$
.24
$$$$
3611.04
$$$$
\text { D }
$$$$
.25
$$$$
3604.10
$$$$
\text { E }
$$

.31

3597.43

A

.22

$357^{R} .89$

E

.34

3571.99

c) $\tau_{y} \rightarrow \tau_{z}$

1791.1
1758.2
1724.5

1.5

1791.13

B

1.0

1758.05

A

1.5

1726.55

B 
Table 13

Measured and Calculated Chlorine ENDOR Transitions of the $3_{\pi \pi^{*}}$ State of Paradichlorobenze (X Trap)

Measured Trequency in MH. (.$\pm \cap 5)$ Calculated Frequency $\tau_{x} \rightarrow \tau_{z}$ Manifold

${ }^{35}{ }_{\mathrm{Cl}} \quad 32.06 ; 32.96 \quad 31.56-33.03$

37C1 25.12; 26.0ก 2h.0! - 26.09

$$
\tau_{x} \rightarrow \tau_{y} \text { Manifold }
$$

${ }^{35} \mathrm{Cl} 31.75 ; \quad 33.13 \quad 31.53-32.94$

${ }^{37} \mathrm{Cl} 24.94 ; 26.19 \quad 24.79-25.90$ 
$-151-$

Table 14

Contributions to the ChIorine Nuclear

Quadrupole Coupling Constant

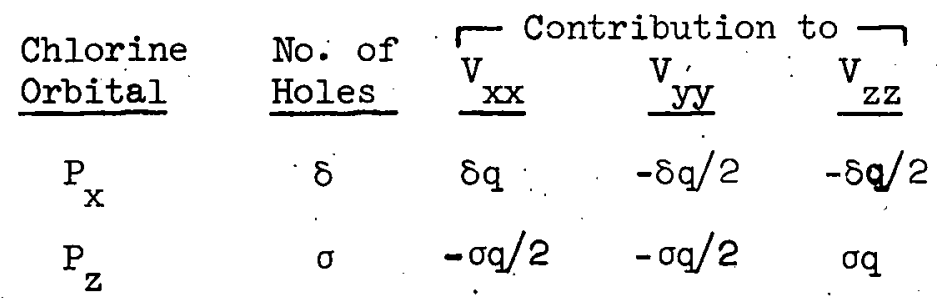

Total Contribution

$$
\begin{aligned}
& \mathrm{v}_{\mathrm{xx}}=(\delta-\sigma / 2) \mathrm{q} \\
& \mathrm{v}_{\mathrm{yy}}=-1 / 2(\delta+\sigma) \mathrm{q} \\
& \mathrm{v}_{\mathrm{zz}}=(\sigma-\delta / 2) \mathrm{q}
\end{aligned}
$$




\section{The PMDR Spectrum of Paradichlorobenzene}

The experimental technique is essentially the same as that used in the ODMR experiments except that the phosphorescence spectrum is scanned while saturating one of the three microwave transitions. The microwave field is amplitude modulated and the resulting time-dependent change in intensity of the phosphorescence converted to DC by means of a $1 n n k$-in amplifier. With this technique only the change in emission of two of the three triplet levels is detected while saturating any one of the three microwave transitions.

In addition to the previously reported exciton phosphorescence 71,97 of $\mathrm{pDB}$ (origin $=27890 \mathrm{~cm}^{-1}$ ), emission from a shallow trap which will be referred to as the $\mathrm{x}$ trap (origin $=27868 \mathrm{~cm}^{-1}$ ) and a deep trap which will be referred to as the y trap (origin $=27807 \mathrm{~cm}^{-1}$ ) was observed. The DDB sample was found to be extremely sensitive to its recent thermal history. In order to observe the weak exciton phosphorescence it was necessary to cool the sample slowly to the temperature of liquid helium over a three hour period. The emission of both the exrition and the $x$ trap was observed at $4.2^{\circ} \mathrm{K}$ with approximately equal intensity. Upon cooling the sample to $1.3^{\circ} \mathrm{K}$ only the $\mathrm{x}$ trap emission was obocrved. If the lempcrature of the pDB crystal was lowered to $4.2^{\circ} \mathrm{K}$ rapidly (approximately 20 minutes), exciton emission was not observed, but the emission from the $y$ trap was observed in addition to that from the $x$ trap. Upon cooling the sample below $4.2^{\circ} \mathrm{K}$, the intensity of the $\mathrm{x}$ trap emission increased while the intensity of the $y$ trap emission decreased until at $1.3^{\circ} \mathrm{K}$ only the $\mathrm{x}$ trap emission was observed. The $\mathrm{y}$ trap emission is 
believed to be due to triclinic inclusions in the monoclinic pDB lattice. This was tested by preparing a sample of pDB which contained approximately. 0.01 mole percent paradimethibenzene as an impurity. Francis ${ }^{121}$ has found from laser man studies that this impurity forces the pDB to remain in its triclinic crystaliine form. Since the same phosphorescence origin as well as the same ESR transition frequencies ( $\pm 20 \mathrm{MHz}$ ) were observed for both the $y$ trap and the triclinic form of $\mathrm{pDB}$, the $\mathrm{y}$ trap is due to triclinic inclusions. An example of the usefulness of the PMDR technique is the resolution of the emission from the $x$ and $y$ traps. In Figure 46 is shown the first $300 \AA$ portion of the phosphorescence spectrum of $\mathrm{pDB}$ at $1.95^{\circ} \mathrm{K}$ which is composed of both $\mathrm{x}$ and $\mathrm{y}$ trap emission. Since the FSR transition frequencies are slightly different for the two traps, the contribution to the phosphorescence spectrum due to only one of the traps may be obtained by saturating the ESR transition associated with the particular trap molecules while monitoring only the component of the phosphorescence emission that is changing in amplitude at the modulation frequency. An example of the spectra obtained from this experiment is shown in Figure 47. The emission from the y trap is no longer observed and the phosphorescence spectrum consists of only emission from the $\mathrm{x}$ trap.

In addition to resolving the emission from different molecular sites in the crystal, the use of the PMDR technique simplifies the analysis of the vibrational structure of the phosphorescence, and in favorable cases gives the orientation of the electron spin-spin tensor and the spacial symetry of the excited state.

The phosphorescence spectrum of the $x$ trap of pDB at $1.35^{\circ} \mathrm{K}$ obtained using $10 \mu$ slits is shown in Figure 48. In order to obtain a reasonable 


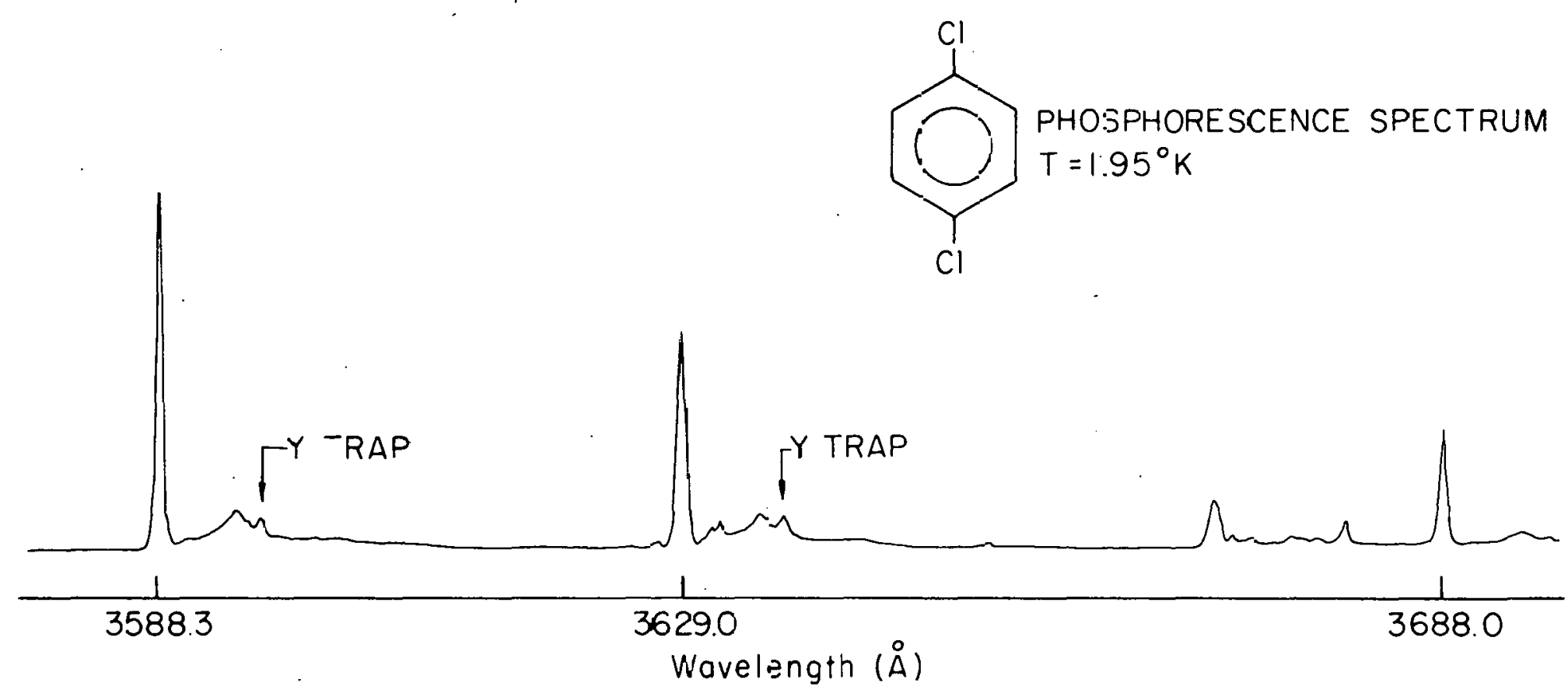

XBL $711-5 j$

Figure 46

Phosphorescence spectrum of paradichlarobenzene 


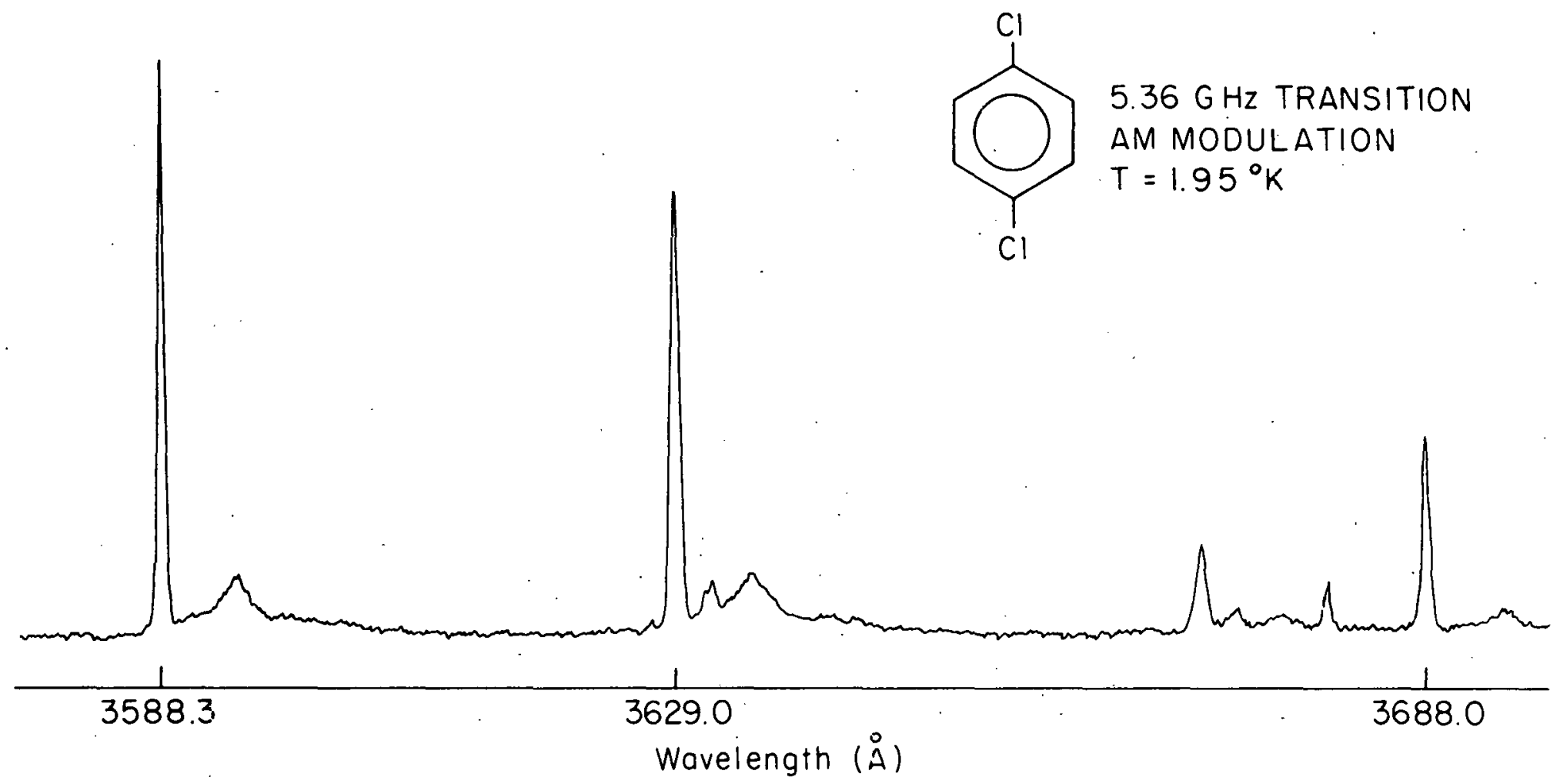

$X B L \uparrow 11-56$

Figure 47

PMDR spectrumof the $X$ trap emission of paradichlorobenzene 
signal to noise ratio for the PMDR experiments, it was necessary to use $50 \mu$ slits resulting in a lower resolution spectrum. In Figure 49 the phosphorescence spectrum using 50 slits (49a) and the PMDR spectra obtained with the same slit width while saturating the $\tau_{x} \rightarrow \tau_{z}$ (49b), $\tau_{x} \rightarrow \tau_{y}(49 c)$ and $\tau_{y} \rightarrow \tau_{z}(49 d)$ transitions are shown. All of the vibronic transitions increase in intensity for each of the PMDR experiments. Since a lock-in amplifier was used, any decrease in intensity while monitoring the emission to a vibrational level of the ground state would have caused the PMDR transition to go negative rather than positive. 'the relative increase in intensity of the vibronic band at $0-0+1583 \mathrm{~cm}^{-1}$ (denoted by * in Figure 49) compared to the origin is striking. The relative increase in intensity of the vibronic band is greatest while saturating the $\tau_{x} \rightarrow \tau_{y}$ transition (49c) while the relative incrense in Intensity of the origin is greatest for the $\tau_{x} \rightarrow \tau_{z}$ transition (49b). The other vibrations that have been assigrled as a behave in a manner similar to the origin and therefore it is clear that the vibration at $0-0+1583 \mathrm{~cm}^{-1}$ is not an a vibration as previously assigned ${ }_{\mathrm{g}}^{71,97}$ since. all vibrations with the same symmetry should maintain a constant intensity rat10 within all three $\mathrm{PMDR}$ spectra. This vibration is therefore assigned $b_{3 g}$ symmetry since from laser Raman studies both $a b_{3 g}$ and an $a_{g}$ vibration are observed at this energy. 122,123

Analysis of the polarization of the absorption spectrum $\left(\mathrm{S}_{\mathrm{O}} \rightarrow \mathrm{T}_{1}\right)$ of $\mathrm{pDB}$ shows that the transition is primarily out-of-plane polarized which has been interpreted by Castro and Hochstrasser ${ }^{71}$ as implying $B_{2 u}$ symetry for the excited state. The three levels of a $B_{2 u}$ triplet transform as 


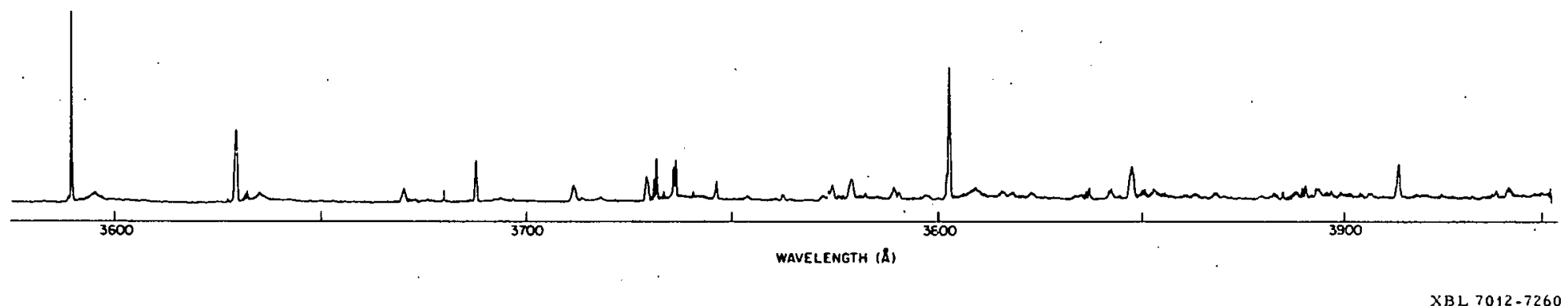

Figure 48

High resolution phosphorescence spectrumof the $X$ trap emission of paradichlorobenzene at $1.3^{\circ} \mathrm{K}$ 
(o)

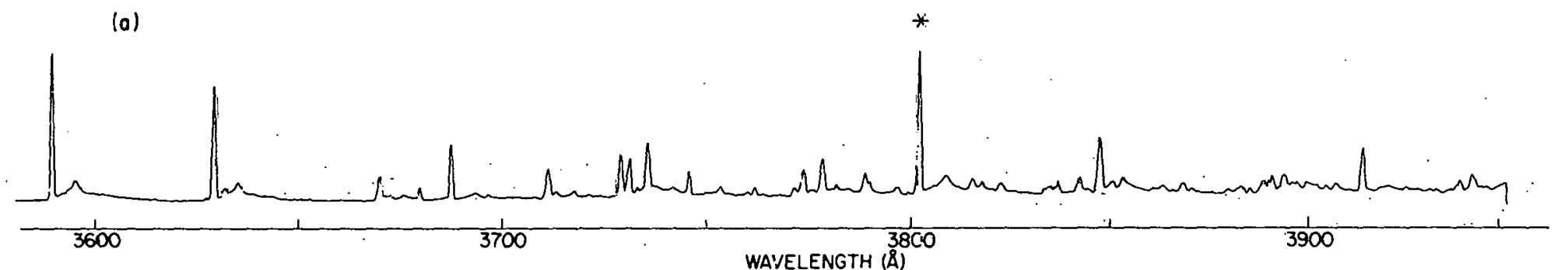

(b)

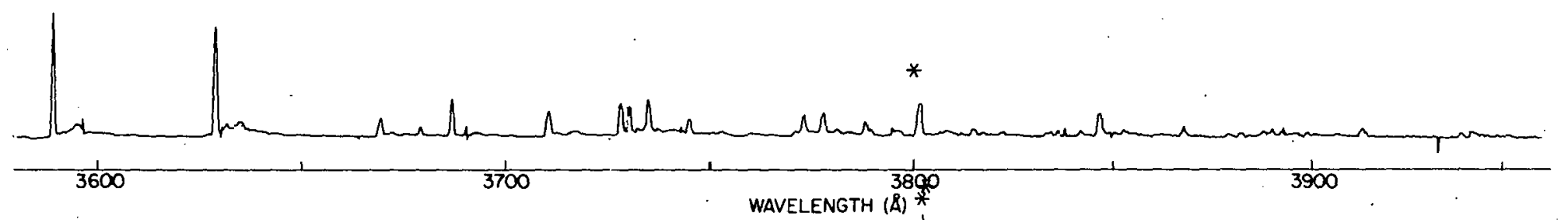

(c)
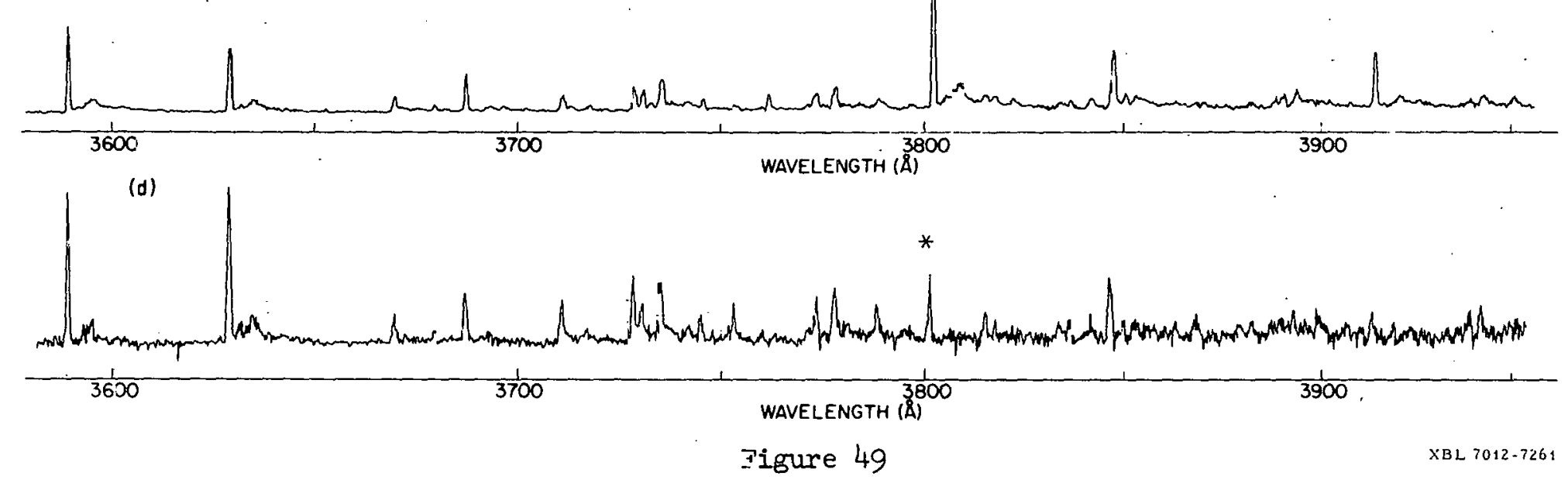

Phosphorescence spectra (a) and PMDR spectra obtained while saturating the $\tau_{x} \rightarrow \tau_{z}(b)$,

$\tau_{x} \rightarrow \tau_{y}(c)$ and $\tau_{y} \rightarrow \tau_{z}$ (d) zero fie-d transitions of paradichlorobenzene (X trap) at $1.3^{\circ} \mathrm{K}$. 


$$
\begin{aligned}
& \tau_{x}=-B_{3 g} \times B_{2 u}=B_{1 u} \\
& \tau_{y}=B_{2 g} \times B_{2 u}=A_{u} \\
& \tau_{z}=B_{I g} \times B_{2 u}=B_{3 u}
\end{aligned}
$$

As is shown in Table 15 the emission to the $b_{3 g}$ vibrations must originate primarily from the $\tau_{y}$ triplet level since the emission is primarily $x$ polarized. Since the largest relative intensity of the $b_{3 g}$ vibrations are obtained upon saturation of the ESR transition at $\sim 3.6 \mathrm{GHz}$ (see Figure 49c) that is between the frequency of the other two ESR transitions ( 5.3 and $1.7 \mathrm{GHz}$ ), the $\tau_{\mathrm{y}}$ level must be the lowest or middle triplet energy level. The possibility of the $\tau_{y}$ level as being either the highest or middle energy level is ruled out since this would require a larger value of $E$ and correspondingly smaller value of $D$ than is theoretically reasonable. As discussed in the previous section, the ${ }^{\tau} \mathrm{x}$ level is almost certainly the lowest in energy and therefore the energy of the triplet levels must be ordered,

$$
\mathrm{E}_{z}>\mathrm{E}_{\mathrm{y}}>\mathrm{E}_{\mathrm{x}}
$$

The fact that the intensity of the entire phosphorescence spectrum increases for all three PMDR experiments requires that the triplet level which emits to the ground state with the fastest radiative rate constant have the smaller initial population. This further requires that the population and radiative rate constants of the triplet levels be ordered respectively $\mathbb{N}_{x}>N_{y}>N_{z}$ and $K_{z}>K_{y}>K_{z}$ or vice versa, 
Since there is a moderate amount of in-plane polarization, the contribution to the radiative rate constants of the three triplet levels due to other additional spin-orbit coupling must be included. The resulting polarization of the emission from the triplet levels to the ground vibrational states is given in Table 15. The emission intenoity from any one triplet level may be written,

$$
I_{i}=N_{i} \sum K_{i}^{j}
$$

where $I_{i}$ in the intensity of emission from triplet level $\tau_{i}$ with population $N_{i}$, and $K_{i}^{j}$ is the radiative rate constant for emission from $\tau_{i}$ with $j$ polarization. The phosphorescence that is monitored is a sumation of the emission from each of the triplet levels to a particular vibronic band (i.e., $a_{g}, b_{2 g}$, or $b_{3 g}$ ). The intensity of the phosphorescence in terms of the symetry of the vibronic level that is monttored for the case of $a B_{z u}$ triplet is given by

$$
\begin{aligned}
& I_{a_{g}}-N_{x} K_{x}^{Z}+N_{z_{1}} K_{r}^{x} \\
& I_{b_{2 g}}=N_{x} K_{x}^{x}+N_{y} K_{y}^{y}+N_{z} K_{z}^{Z} \\
& I_{b_{3 g}}=N_{x} K_{x}^{\mathrm{N}}+N_{y} K_{y}^{X}
\end{aligned}
$$

Upon saturation of one of the three microwave transitions, the population of the two levels connected by the microwave field are equalized (see Section II). The change in intensity of the phosphorescence is proportional to 


$$
\Delta I \approx\left(N_{i}-N_{j}\right)\left(K_{j}-K_{i}\right)
$$

However, if only the emission to a particular vibronic band is monitored, the emission from both of the levels that are connected by the microwave field may not be observed. In this case, the change in intensity of the phosphorescence when triplet levels $i$ and $j$ are connected by the microwave field and the emission from level $i$ is not monitored is

$$
\Delta I \approx\left(N_{i}-N_{j}\right) K_{j}
$$

In Table 16 the change in phosphorescence intensity is predicted for the ${ }^{3} \mathrm{~B}_{2 \mathrm{u}}$ state of $\mathrm{pDB}$ upon saturating any one of the three zero field transitions while monitoring the emission to a particular vibration of the ground state with $a_{g} b_{2 g}$ or $b_{3 g}$ symmetry. With the assumption that the populations are ordered $\mathrm{N}_{x}>\mathrm{N}_{\mathrm{y}}>\mathrm{N}_{\mathrm{z}}$ end that all radiative rate constants from $\tau_{z}$ are greater than those from $\tau_{y}$ which are greater than those from $\tau$ ' the change in phosphorescence is predicted to increase for six of the cases but decrease for the other three. Since the phosphorescence was observed to always increase, this assignment is clearly incorrect. However, if the molecule is distorted from $D_{2 h}$ to $C_{2 h}$ symetry, the distinction between the $x$ and $z$ axes is lost and the triplet levels transform as

$$
\begin{aligned}
& \tau_{x}=B_{g} \times A_{u}=B_{u} \\
& \tau_{y}=A_{g} \times A_{u}=A_{u} \\
& \tau_{z}=B_{g} \times A_{u}=B_{u}
\end{aligned}
$$


and the vibrations $a_{g}, b_{2 g}$ and $b_{3 g}$ transform as $a_{g}, a_{g}$ and $b_{g}$ respectively. The intensity of emission to the vibrational levels in this case is

$$
\begin{aligned}
& I_{a_{g}}=N_{x} K_{x}^{x z}+N_{y} K_{y}^{y}+N_{z} K_{z}^{x z} \\
& I_{b_{g}}=N_{x} K_{x}^{y}+N_{y} K_{y}^{x z}+N_{z} K_{z}^{y}
\end{aligned}
$$

In table 17 the change in intensity predicted for the distorted molecule is given. As can be seen in this case, the intensity of the phosphorescence always increases. If the molecule is distorted only two basic types of vibrational structures will be observed since both $a_{g}$ and $b_{a g}$ vibrations transform as $a_{g}$ in $C_{2 h}$. As can be seen in Table 18 the intensity ratio of the vibronic structure of the phosphorescence for the three PMDR experiments shows only two distinct types of vibrations. If the molecule is distorted, the transitions will be equally polarized along the $x$ and $z$ molecular axes. From the values of the squared directional cosines of PDB listed in Table 19, equal polarization along the $\mathrm{x}$ and $\mathrm{z}$ molecular axes gives a polarization ratio along the crystal axis of $I_{c} / I_{b}=i .5$ as compared to the value of $I_{c} / I_{b}=3.4$ for polarization along the molecular $\mathrm{x}$ axis only. The measured polarization ratio of $T_{\mathrm{U}} / I_{\mathrm{b}}$ for the absorption origin in 0.9 .97 and $3.1,71$ which 1 r nul inconsistent with the conclusion that $\mathrm{pDB}$ is distorted in its excited triplet state into a trans configuration.

In summary, the observed $O D M R$ and $P M D R$ spectra of $\mathrm{PDB}$ are consistent with the hypothesis that the excited triplet state of $\mathrm{pDB}$ is a $\pi \pi^{*}$ state and that the symetry of the excited state is $B_{2 u}$. From the assigned orientation of the zero field tensor, the spin density appears to be 
localized primarily on the four carbons that are not bonded to the chlorines. The small value of the chlorine out-of-plane hyperfine element leads to the conclusion that the chlorines do not participate significantly in the excitation.

On the basis of the PMDR spectra and the reduced value of the chlorine muclear quadrupole coupling constant as compared to the value for the ground state, It is hypothesized that the $\mathrm{C}-\mathrm{Cl}$ bonds are bent and that the molecule... possesses $C_{2 h}$ rather than $D_{2 h}$ spacial symmetry in the excited state.

The assignment of the symmetry of the excited triplet state of pDB as a $\mathrm{B}_{2 \mathrm{u}}$ state is somewhat disturbing in view of the small participation of the chlorines in the excitation and the accepted assignment of the first excited triplet state of benzene as a $B_{1 u}$ state.

Therefore two experiments are proposed to remove many of the assumptions made in the preceeding discussion. The measurement of the ODMR spectra in a magnetic field would give the orientation of the principal axis systems of $\mathrm{H}_{\mathrm{SS}}, \mathrm{H}_{Q}$ and $\mathrm{H}_{\mathrm{HF}}$ and in addition permit the measurement of the two in-plane components of the chlorine hyperfine tensor. The use of a polarizer to separate the components of the phosphorescence spectra while performing the PMDR experiments would permit a more detailed analysis of the vibrational stmacture of the phosphorescence and consequently an unambiguous assignment of the symmetry of the excited triplet state of pDB. 
$-164-$

Table 15

Polarization of the Phosphorescence

to the Ground State Vibrational Levels

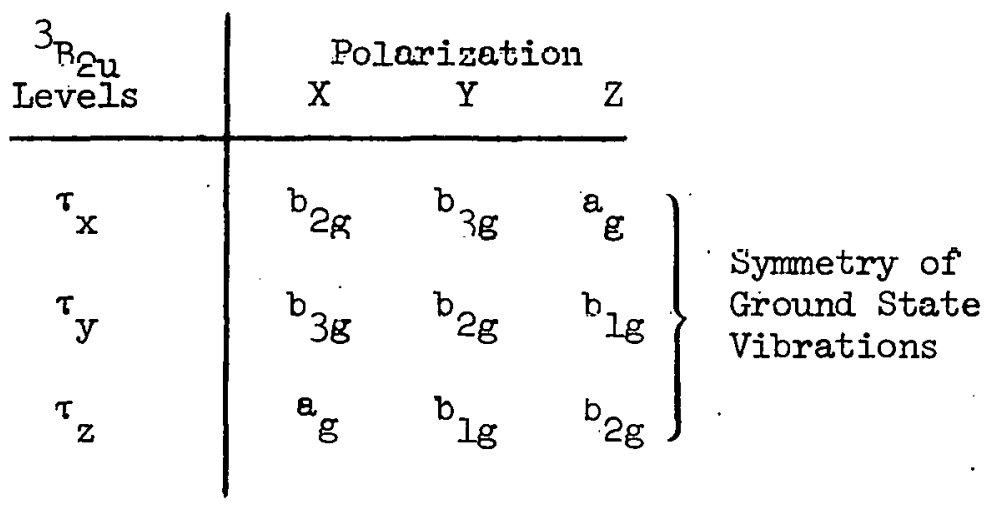


Table 16

Predicted change in intensity of the phosphorescence for a molecule in a ${ }^{3_{2 u}}$ state when monitoring the individual vibronic bonds of the phosphorescence*.

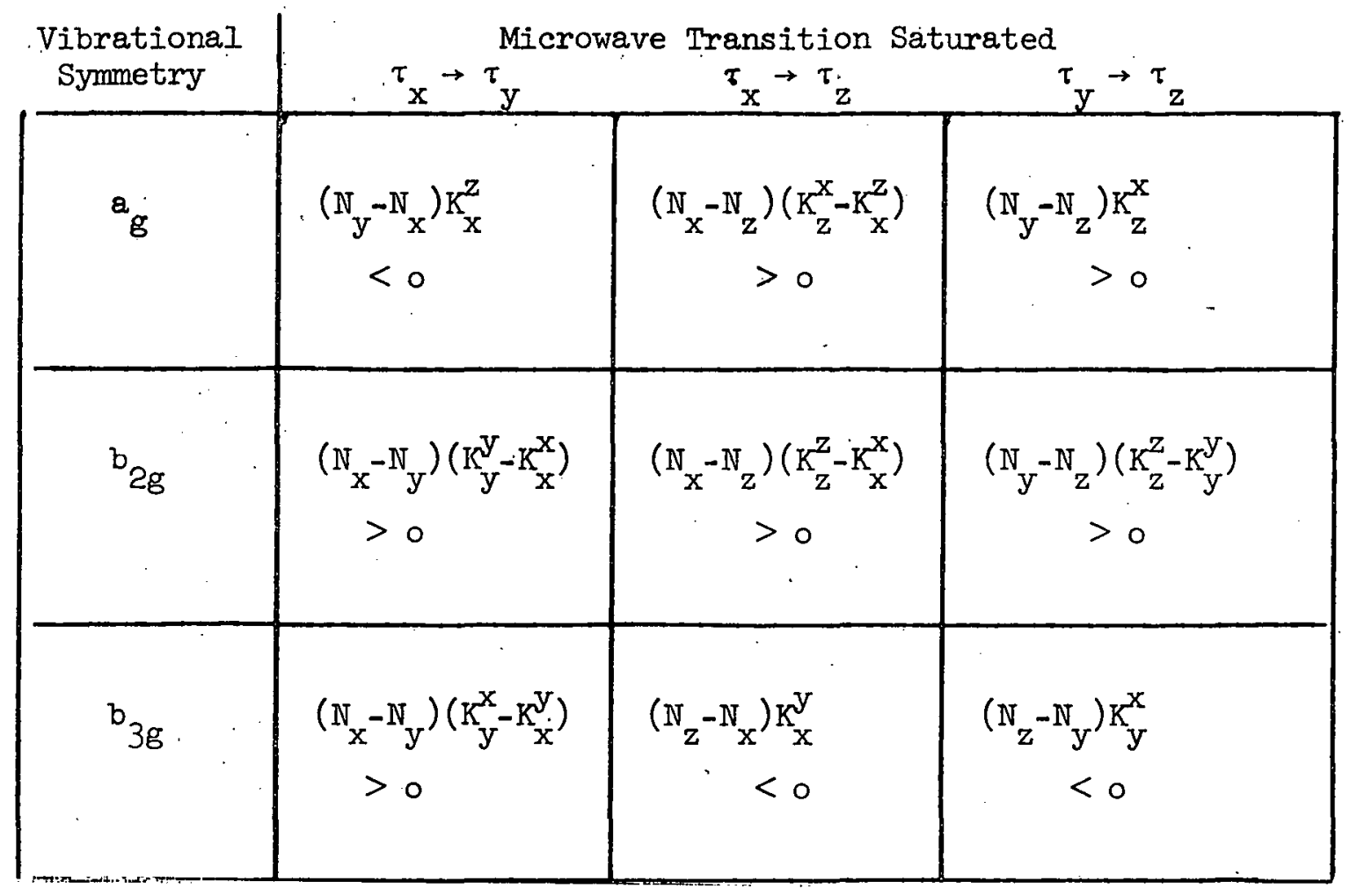

* The change in intensity is based on the assigrment $N_{x}>N_{y}>N_{z}$ and $K_{z}^{i}>K_{y}^{i}>K_{x}^{i}$ 
Predicted change in intensity of the phosphorescence for a distorted $\left(C_{2 h}\right)$ excited state.

\begin{tabular}{|c|c|c|c|}
\hline $\begin{array}{c}\text { Vibrational } \\
\text { Byimuliry }\end{array}$ & $\tau_{\mathrm{x}} \rightarrow{ }_{\mathrm{y}}^{\text {Microwe }}$ & $\begin{array}{l}\text { ve Transition Sat } \\
\tau_{x} \rightarrow \tau_{z}\end{array}$ & $\tau_{y} \rightarrow \tau_{z}$ \\
\hline${ }^{a_{g}}$ & $\begin{array}{c}\left(N_{x}-N_{y}\right)\left(K_{y}^{\mathrm{V}}-K_{x}^{x z}\right) \\
>0\end{array}$ & $\begin{array}{c}\left(N_{x}-N_{z}\right)\left(K_{z}^{X z}-K_{x}^{X z}\right) \\
>0\end{array}$ & $\begin{array}{c}\left(\mathrm{N}_{\mathrm{y}}-\mathrm{N}_{\mathrm{z}}\right)\left(\mathrm{K}_{\mathrm{z}}^{\mathrm{xz}}-\mathrm{K}_{\mathrm{y}}^{\mathrm{y}}\right) \\
>0\end{array}$ \\
\hline${ }^{b} g$ & $\begin{array}{c}\left(N_{x}-N_{y}\right)\left(K_{y}^{x z}-K_{x}^{y}\right) \\
>0\end{array}$ & $\begin{array}{c}\left(N_{x}-N_{z}\right)\left(K_{z}^{\mathrm{Y}}-K_{x}^{\mathrm{y}}\right) \\
>0\end{array}$ & $\begin{array}{c}\left(\mathrm{N}_{\mathrm{y}}-\mathrm{N}_{\mathrm{z}}\right)\left(\mathrm{K}_{\mathrm{z}}^{\mathrm{V}}-\mathrm{K}_{\mathrm{y}}^{\mathrm{Xz}}\right) \\
>0\end{array}$ \\
\hline
\end{tabular}

*The change in intensity is based on the assumption $N_{x}>N_{y}>N_{z}$ and $K_{z}^{i}>K_{y}^{i}>K_{x}^{i}$ or vice verca. 
Table 18

Analirsis of the PMDR Spectra of the X Trap

Phosphorescence of Paradichlorobenzene ( $1.3^{\circ} \mathrm{K}$ )

\begin{tabular}{|c|c|c|c|c|c|c|c|c|}
\hline \multirow[b]{2}{*}{$\vec{v}\left( \pm 5 \mathrm{~cm}^{-1}\right)$} & \multirow[b]{2}{*}{$\Delta \bar{v}$} & \multicolumn{3}{|c|}{$\begin{array}{l}\text { Intensity }{ }^{*} \text { of PMDR } \\
\text { While Saturating }\end{array}$} & \multicolumn{2}{|c|}{$\begin{array}{c}\text { Intensity } \\
\text { Ratio }\end{array}$} & \multirow[b]{2}{*}{ TYPE } & \multirow[b]{2}{*}{ Assignment ${ }^{*}$} \\
\hline & & $\tau_{x} \stackrel{A}{\rightarrow} \tau_{z}$ & $\tau_{x} \stackrel{B}{\rightarrow} \tau^{y}$ & $\tau_{\mathrm{y}} \stackrel{\mathrm{C}}{\rightarrow} \tau_{\mathrm{z}}$ & $\mathrm{A} / \mathrm{C}$ & $\mathrm{B} / \mathrm{C}$ & & \\
\hline 27868 & & 61 & 43 & 77 & 1.42 & 0.56 & $I$ & Trap origin \\
\hline 27823 & 45 & 6 & 7 & 13 & 0.86 & 0.54 & $\frac{1}{I}$ & Lattice $\left(41 \mathrm{~B}_{\mathrm{g}}\right)$ \\
\hline 27556 & 312 & 33 & 32 & 79 & 1.72 & 0.41 & $\bar{I}$ & $b_{2 g}(306)$ \\
\hline 27538 & 330 & 7 & 7 & 10 & 1.00 & 0.70 & $\bar{I}$ & as $(327)$ \\
\hline 27504 & 364 & 8 & 10 & 15 & 0.80 & 0.67 & $I$ & $312+46$ \\
\hline 27247 & 621 & 10 & 20 & 15 & 0.50 & 1.33 & $I$ & $2 \times 312$ \\
\hline 27115 & 753 & 19 & 10 & 26 & 1.90 & 0.38 & $\bar{I}$ & $a_{g}(744)$ \\
\hline 26938 & 931 & 13 & 8 & 23 & 1.63 & 0.35 & $\bar{I}$ & $3 \times 312$ \\
\hline 26808 & 1061 & 17 & 14 & 35 & 1.21 & 0.40 & $\bar{I}$ & $755+313$ \\
\hline 26793 & 1075 & 16 & 12 & 20 & 1.33 & 0.60 & $I$ & $a_{g}(1081)$ \\
\hline 26759 & 1109 & 19 & 18 & 31 & 1.06 & 0.58 & $\vec{I}$ & $\operatorname{ag}(1103)$ \\
\hline 26686 & 1182 & 10 & 7 & 15 & 1.43 & 0.47 & $\bar{I}$ & $a_{g}(1174)$ \\
\hline 26570 & 1298 & 2 & 10 & 1 & 0.20 & 10.0 & $I I$ & b $3 g(1293$ \\
\hline 26502 & 1366 & 4 & 5 & $\overline{8}$ & 0.80 & 0.63 & $I$ & \\
\hline 26487 & 1382 & 12 & 11 & 24 & 1.09 & 0.46 & $I$ & \\
\hline 26453 & 1415 & 12 & 14 & 28 & 0.86 & 0.50 & $I$ & \\
\hline 26285 & 1583 & 17 & 101 & 35 & 0.17 & 2.89 & II & $b_{3 g}(1577)$ \\
\hline 26241 & 1627 & 3 & 15 & 0 & 0.20 & $\infty$ & II & $15 \varepsilon_{3}+46$ \\
\hline 26054 & 1814 & 5 & 7 & 13 & 0.71 & 0.54 & $I$ & \\
\hline 26016 & 1852 & 4 & 8 & 16 & 0.50 & 0.5 & $\bar{I}$ & \\
\hline$\varepsilon 5980$ & 1888 & 13 & 32 & 33 & 0.41 & 0.97 & $\bar{I}$ & \\
\hline 25695 & 2173 & 5 & 10 & 14 & 0.5 & 0.71 & $I$ & \\
\hline 25542 & 2326 & 6 & 31 & 17 & 0.19 & 1.82 & II & $1583+753$ \\
\hline
\end{tabular}

* Ground state values are given in parentheses (See reference 123). 
$-168-$

Table 19

Squared Directional Cosines of the Molecular Axes with Respect to the Crystallographic Axes ${ }^{*}$

Purad1chlorobenzene $\left(133^{\circ} \mathrm{K}\right)$

Molecular Axis Crystallographic Axis

$\begin{array}{cccc} & a & b & c \\ X & 0 & 0.231 & 0.77^{8}\end{array}$

$\begin{array}{llll}Y & 0.472 & 0.412 & 0.116\end{array}$

$\begin{array}{llll}7 . & 0.537 & 0.357 & 0.106\end{array}$

*From reference 71 . 


\section{ACKNOWLEDGEMENTS}

I would like to express sincere appreciation to Professor C. B. Harris for his constant support and guidance throughout the course of this work. I am indebted to him for having shown me many fascinating and exciting aspects of science.

I would also like to thank Professor M. A. El-Sayed (University of California at Los Angeles) for supplying the samples of 8-chloroquinoline and 2-3 dichloroquinoxaline, and Professor A. H. Maki (University of California at Riverside) for supplying one of the pyrazine samples.

I would also like to express appreciation to all of Professor Harris's graduate students and research associates for many stimulating discussions and especially Dr. A. H. Francis for his lucid explanations of the fundamentals of optical spectroscopy.

Thanks are also due to the many technical support groups at both the Chemistry Department and the Lawrence Radiation Laboratory and in particular the Chemistry Department machinists for their ability to fabricate equipment from only rudimentary instructions.

Finally I would like to thank my wife Barbara for her constant encouragement and support throughout the course of this work. 


\section{APPENDIX}

Program ODMR

\section{A. Description}

This Fortran IV program written for the CDC 6600 computes the electron magnetic dipole allowed transition frequencics and probalilities as well as the energy levels for a spin system consisting of the triplet electrons and an arbitrary number of nuclei in the absence of an external magnetic field. The present form of the program accepts up to 4 different nuclear spins and will solve a spin Hamiltonian matrix up to $48 \times 48$. The number of nuclear spins may be increased to any necessary number by changing the dimensions of the appropriate matrices.

The program calculates the spin Hamiltonian matrix by evaluating the individual contributions from $\mathrm{H}_{\mathrm{SS}}, \mathrm{H}_{\mathrm{Q}}$ and $\mathrm{H}_{\mathrm{HF}}$ (see section III). The basis states of the spin Hamiltonian are the eigenstates of $S_{7}$ for both the electron and nuclear spins. In order to allow for noncoincidence of the axis systems that diagonalize $\mathrm{H}_{\mathrm{SS}}, \mathrm{H}_{\mathrm{Q}}$ and $\mathrm{H}_{\mathrm{HF}}$, a rotation of the nuclear quadrupole and/or nuclear hyperfine Hamiltonian axis systems about the $\mathrm{y}$ axis of $\mathrm{H}_{\mathrm{SS}}$ is provided.

The individual matrix elements of $\mathrm{H}_{S S}, \mathrm{H}_{Q}$ and $\mathrm{H}_{\mathrm{IL}}$ are evaluated and collected into groups depcnding on the uperators involved, such as $\mathrm{S}_{z} \mathrm{I}_{z}, \mathrm{~S}_{z} \mathrm{I}_{+}$etc. (see coment cards). The completed spin Hamiltonian is then diagonalized by subroutine HDIAG which was written by Marjorie Merwin, M.I.T:, and converted for CDC 6600 operation by Bill Dempster, IRI Berkeley. The matrix multiplication necessary to compute the transition probabilities is performed by the Compass subroutine 
FEMME (also written by Bill Dempster) which is approximately 5.5 times as fast as an equivalent Fortran subroutine. The frequencies and intensities, as well as the energy levels they connect, are then printed in order from the highest frequency transition to the lowest. In addition, the transitions are collected into groups differing in frequency by

$0.05 \mathrm{MHz}$ (see cards A-11 and A-279) and the weighted average of the transition frequencies and the sumation of their intensities printed.

ENDOR transitions due only to the electron magnetic dipole operator are sorted into the triplet manifold in which they occur and also printed.

An option is provided to suppress printing of the matrix elements of the total spin Hamiltonian (OPI) as well as an option which permits only the weighted average of the transition frequencies to be printed (OP2).

B. Input Variables

Use one set of data cards for each calculation. For a definition of the terms used see section III.

CARD \#I (Format 3F10.3)

\begin{tabular}{|c|c|}
\hline $1-10$ & $\mathrm{x}$ \\
\hline $11-20$ & $Y$ \\
\hline $21-30$ & $\mathrm{z}$ \\
\hline
\end{tabular}

CARD \#2 (Format 3II)

Col 1 Number of nuclear spins

Col 2 If set equal to zero, the input spin Hamiltonian is not printed

Col 3 If set equal to zero, the complete list of transition frequencies is not printed 
CARD \#3 (Format 7F10.3, 2F5.0)

This card is necessary for each nuclear spin

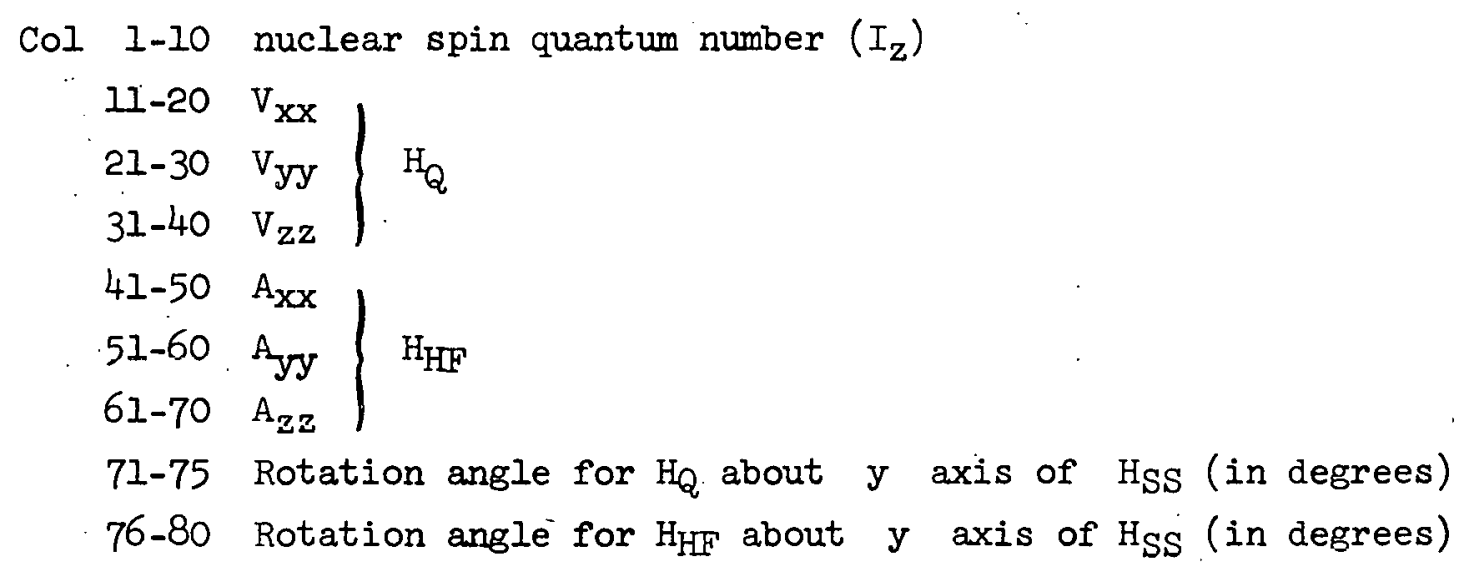

6789 Card 
D.RIIGRAM OCMR (INPIJT, CUTPUIT, TAPFS= IN!PUT )

OIMEISTICA! $\triangle$ ! (48), A $(48,48)$, TKI 48, 48), FIVA $(48,49)$

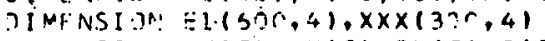

D IMFNSISTN $X \times(5), Y A(5), Z M(=), 8(5), F(5), P H(5), \times(5), J 2(5), K 2(5), I Y(5)$

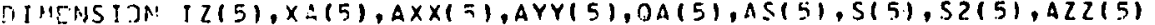

I YINSIS:N $C(E), C .2(5), T H(5), 0(5)$

EQUTVAL, ENICE $(A, \times \times \times),(E !, T K)$

E.F'AL JZ, K?

$F F=S O F+1 ?$.

$P I=17 ? 16 ? 20773250.4 ? 055 \mathrm{cA}$

$F \& S=0 . r 1$

1) CPNit I Aille

C.ALI SFCONO (ZAD)

F.FAO 720, XY(1),YM(1), 7M(1)

IF (ENF, 7$) 710$, 年

20. CUNTINUE

ก1, $3 r \cdot 1=1,1: \cdots$

A (i.) $=n . r$

30 C CART INUE

: 4 ai $L=1,5$

$12(.1)=$.

40. CONT INIIE

no $50 \quad I=1$, 600 .

ก! $50 \quad \mathrm{~J}=1,4$

$+1(.1 ;, 1)=6.6$

50 CMMTIniUe.

$x(1)=1$

$T Y(1)=3$

$n=-3 \div 2 M(1) / 2$.

$[=(Y M(1)-X M(1)) / 2$.

P.EAO .73C, $\Lambda, 1, P 1, \cap \cap 2$

$N 1=N+1$

nn $60 \quad I=2, N 1$

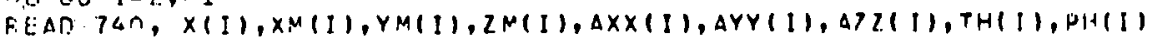

$T H F=\left(T H(I), ! R_{0},\right) * P !$

$P H P=(D H I I) / I R \cap .1 \neq P I$

$S(I)=S I$ in $(T H R)$

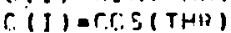

$P(I)=S I N(r H D)$

$F(I)=C, \cap S(P H D)$

$S 2(1)=(R(I)) *(R(1))$

$(2(I)=(F(I)) *(F(I))$

$x A(I)=(x(I))+(x(1))+x(1)$.

$\theta(I)=Z M(I)$

If (OI I) . FO.O.

$D A(1)=.0(1) /(A *(X(1)) * 2-4 *(X(1)))$

$A S(])=(X M(I)-Y M(I)) / Z M(I)$

60 CONTINIUF

$N Z=3$

nO $70 \quad I=2, N !$

$Y Y(I)=2 *(X \mid I))+i$

$N Z=N Z \div|Y| 1]$

7.0 CCNTINIJE

IF (DPI.FG.A) GO TO 80

PPINT 75n, NI.NZ

89 CONTINUE

กח on $I=1, N$.

nO $90 \mathrm{~J}=1 . \mathrm{NZ}$ 


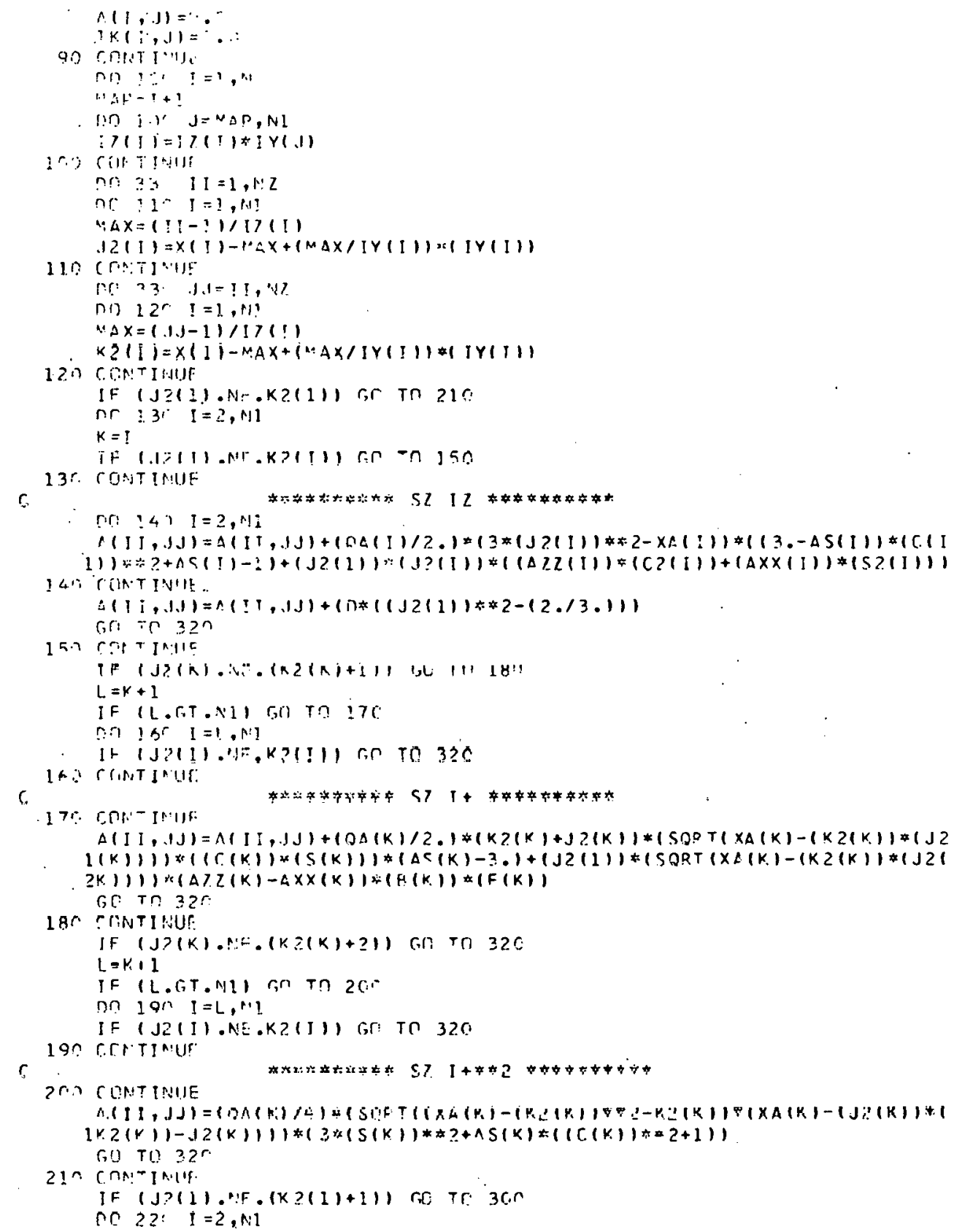




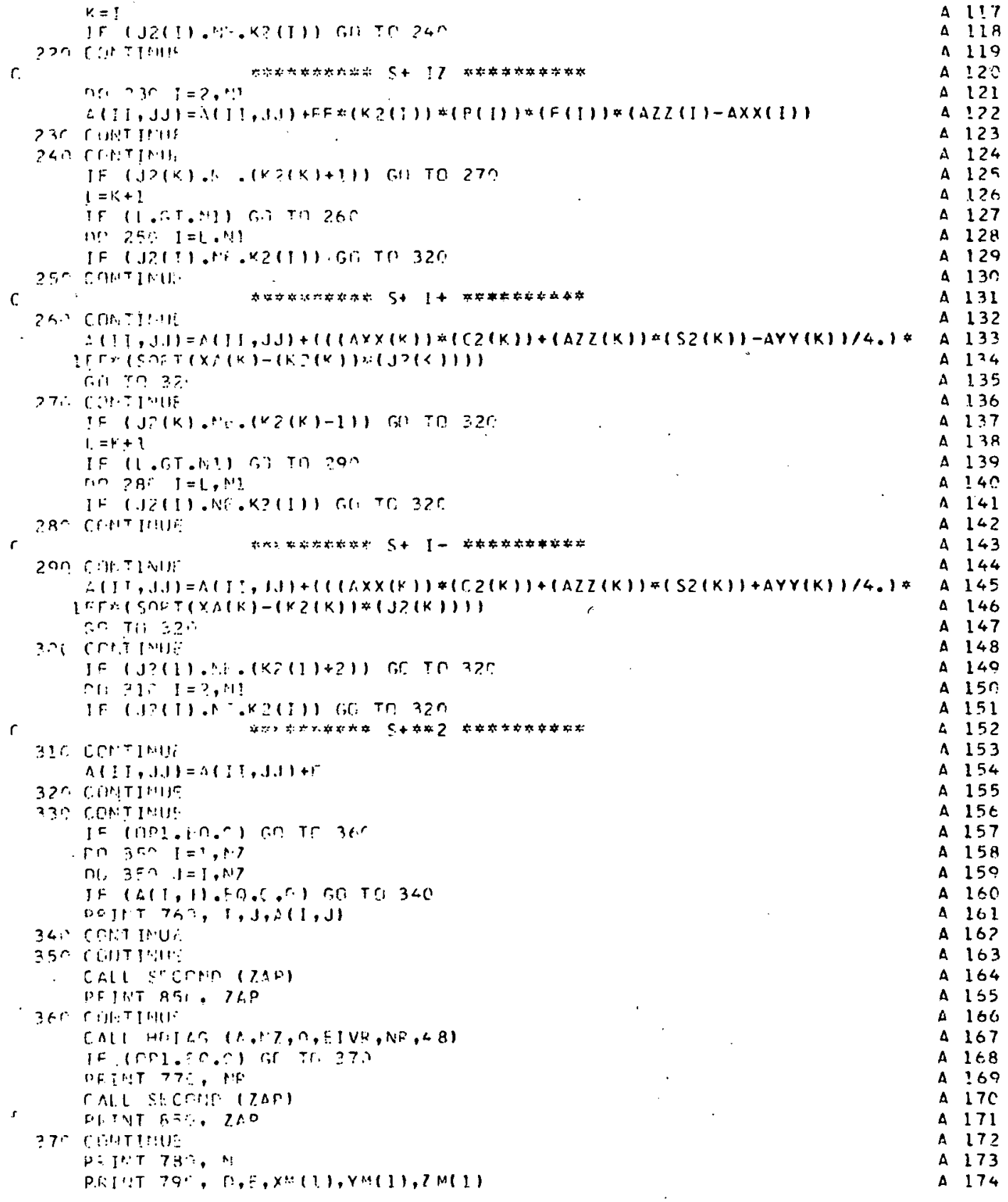

A 117

$\Delta$ í

A 119

A 120

A 121

$4 \quad 122$

$\triangle 123$

A 124

A 125

A 126

$\triangle 127$

A 128

A 129

a $13 n$

A 131

A 132

A 133

A 124

A 135

4136

A 137

A 138

A 139

A 140

A 141

A 142

A 143

A 144

A 145

A 146

A 147

A 148

A 140

A 150

A 151

$4 \quad 152$

$\wedge 153$

A 154

A 155

A 156

A 157

A 158

A 150

A 160

A 161

A 16?

A 163

A 164

A 155

A 160

$\triangle 167$

A $1 \in 8$

A 169

$\triangle 17 C$

A 171

A 172

A 173

A 174 
nO 2 Rח $I=?, N I$

$I=I-1$

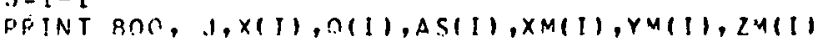

POINT BIr, AXX(1),AYY(I),A TZ(I),TH(I), PH(I)

3.8 CONT INUE.

POI I.

ni) $39 \mathrm{O} \mathrm{I}=\mathrm{I}, \mathrm{NZ}$

PEINT R37, I, A(I, !)

390 C.MHT I RiUE

nก $40, \because \quad I=1, N Z$

$\Delta, I I)=A(I, I)$

400 CONT INUE

$N 3=N Z / 3$

$N 2=2 * N 3$

$00410 \quad I=1, N$ I

$\mathrm{DO} 4 \mathrm{l}, \mathrm{J}=1, \mathrm{NZ}$

$\Delta(1, J)=C$ ?

410 CCNT IPUS

Dก $42 r: \quad 1=1, N 3$

$J=1+112$

$A(1, T)=1$.

$A(1, J)=-1$.

420 C. IINTI INUTE

DO $430 \quad 1=1, N 2$

$J=113+1$

$\Delta(I, J)=F F$

430 CONT IPIUE

C.ALL FEMNE (L, EIVR, TK, NZ, NL, 48 )

DO $4<C . \quad \mathrm{I}=1, \mathrm{NZ}$

ก) $44 ?^{\prime}, J=1, N 7$.

$A(I, J)=r$ I $\operatorname{VF}(J, I)$

440 CONTINUE

DO $45 !: I=1, N 2$

กि 4 हः $J=1, N 2$

EIVF $(I, J)=A(I, 11$

$A(I, J)=C \cdot \cap$

450 R.NNTIN!UF

CALI. FE AME (EIVP, TK, A,NZ,NZ, 48)

DO $48,2 \quad I=1, \mathrm{MI}$

nก $46, \quad t=!, N 7$

$A(I, J)=A B S(A(I, J) * A(J, 1))$

4HO CINT INIIE

CALL SECUND (ZAP)

PRINTT BSก, ZAP

I I $=\mathrm{n}$

D0 $500 \mathrm{I}=1, \mathrm{~N} . \mathrm{2}$

$J=I+1$

IF (J.TT.NL) GDTח 490

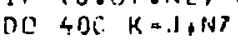

IF $(A(I, K) . L E .1 . C E-9)$ तO TC 470

$I I=I I+1$.

If $(11.07 .599)$ on TO 510

El(II, I) =ARS $(A I(1)-A I(K)$

$F 1(11,2)=A(1, K)$

F. $1(1,3)=1$

F $1(1,4)=K$

470 CONFIHULE

480 CONTINUE

A 175

A 176

$4 \quad 177$

A 178

A 179

4 18?

A 191

A 192.

A 183

A 184

4185

$\triangle 186$

A 181

A 18R

A 189

$\triangle 190$

4 19!

ค 192

A. 197

4194

$\triangle 195$

A 196

A 197

A 149

- 199

A 2C?

A 201

4 2?:

A 203

A 204

$\Delta \gg 5$

A 206

A 207

$\triangle \quad 258$

$\triangle 209$

A 210

$\wedge \geq 11$

A 218

4213

$\Delta \geq 14$

A 215

A 216

A 217

4214

$\triangle 2.19$

4221

4 2? 1

A 2??

4 223

A 224

$\triangle 225$

A $2: 5$

A 227

A 238

$A>>9$

4 231

$\triangle \quad 231$

A 232 


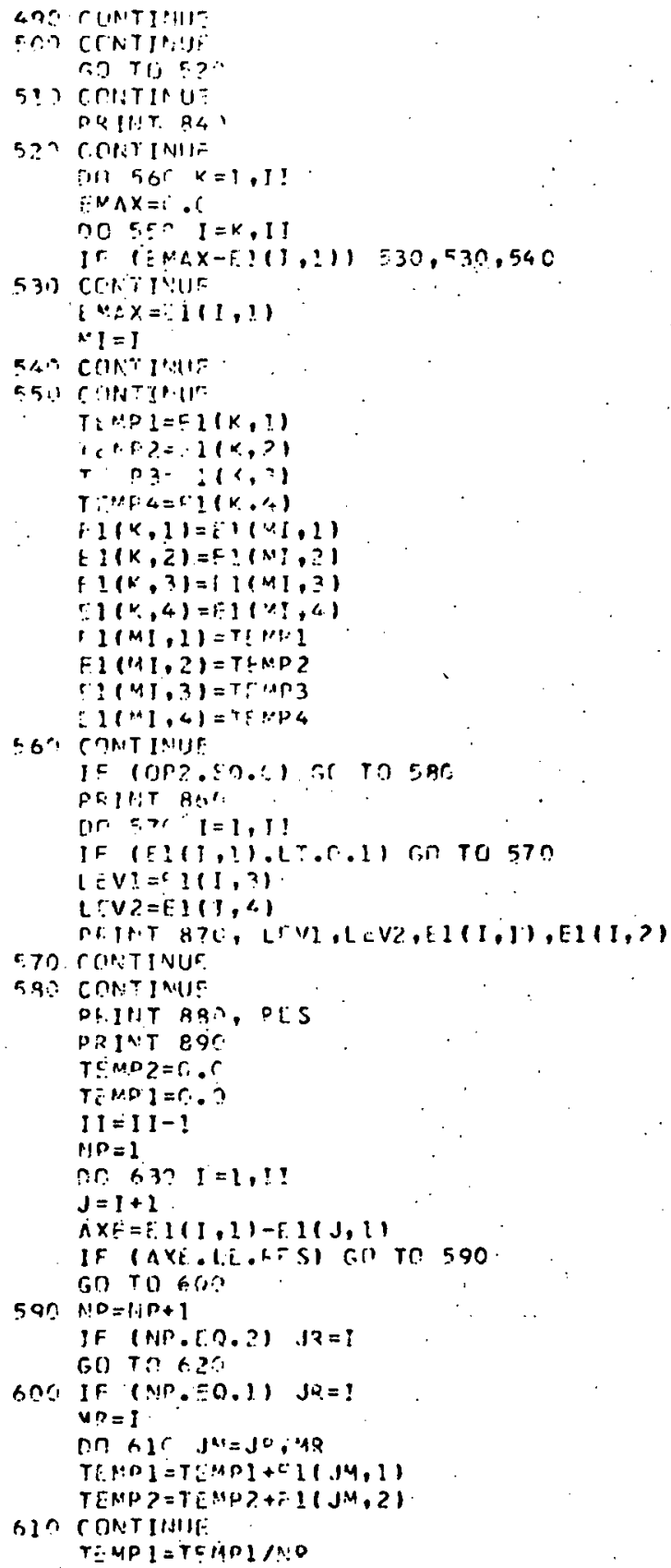




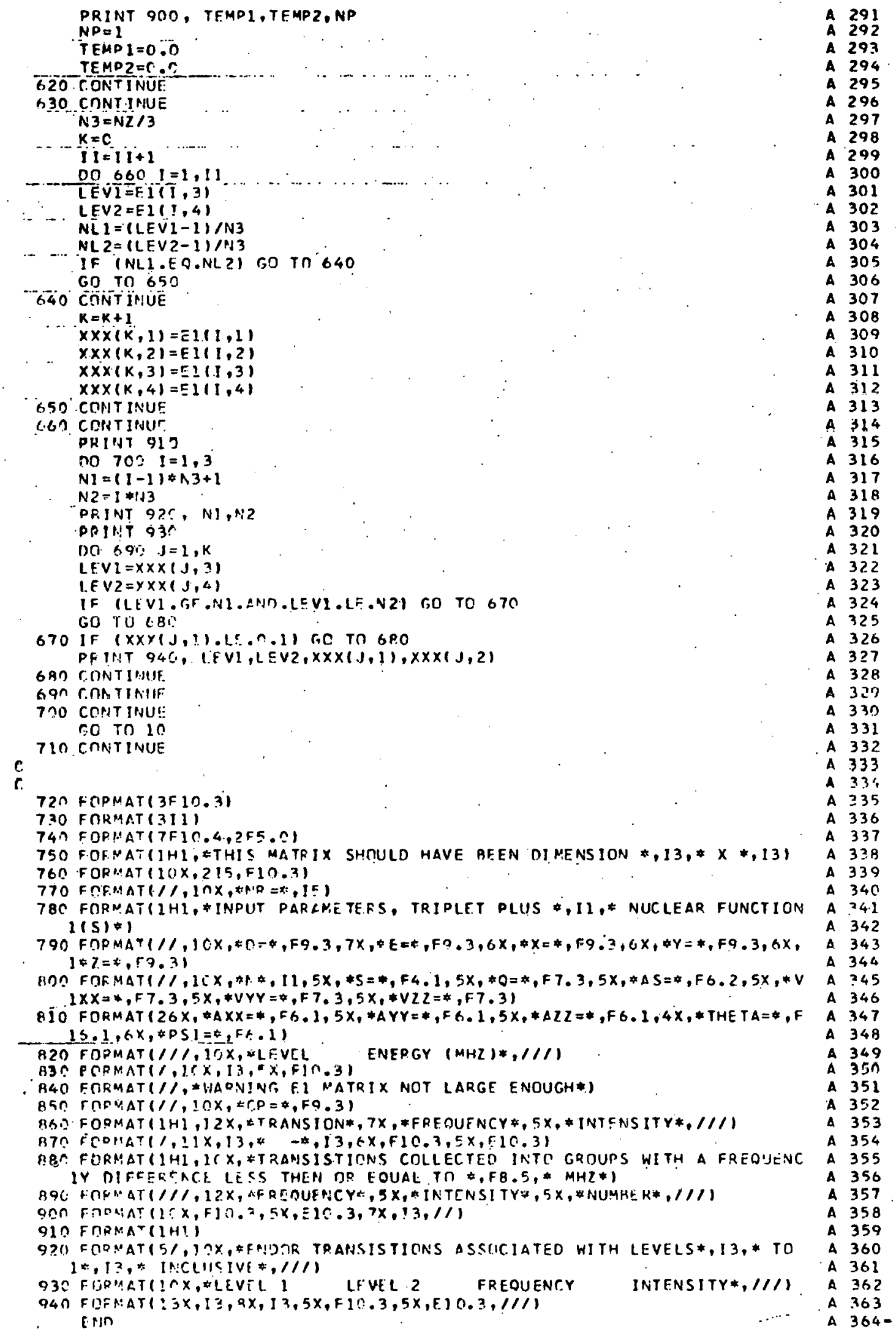


FORTRAN IV SUBRTUTINE HDIAGIH,N, IFGEN,U,N:, NOIMEN)

DIMF NSIIJN H(NOINEN, 11 , U(PIDI4EN, $11, \times(49), 101481$

IF IIFG:NI 50,2r:50

1 ? C.? $42.1=1, r$ !

on $4 C J=1, N$

If $(I-7) 3 r, ?), 30$

$20 \cup(1, j)=1$. ?

GO T. 4 ती

$30 \mathrm{U}(1, \mathrm{~J})=0, \mathrm{n}$

40 rant In!U!

$N 2=(2 * 1 !) / 3$

$50 \quad N R=0$

If $(N-1)<3 n, 43 C, 6 C$

$6 \cap N M I 1.0 N-1$

DC PD $I=1, N ! 14 ! 1$

$x(1)=\mathrm{L} .0$

$I P L I=I+1$

[io an $J=I P L ! N$

KILt: $=I-J$

IF (KILL.ER..H2) GO TO 70

IF. $(X \mid I)-\triangle A S(H(I, J) \mid): 70,70,80$

70 XII) $=A R S(H(I, j))$

$\operatorname{lol}(1)=\mathrm{T}$

BO C.ONT INIJE

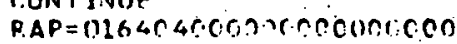

HDTEST $=1$. SE $3 \cap 7$

90 no $12 r_{1} I=1, \mu M I I$

IF $(1-1) 110,11^{n}, 100$

100 If $(x \times A x-x(1)) 12 \%, 120,120$

$1.10 \times 4.4 X=X(1)$

IP $\ V=$ I

$J P I V=\ln (1)$

120 CONT INUE

IF $\left|X^{4} A X\right| 436,4 ? R, 130$

130 IF (HOTEST) $152,150,140$

142 IF (XMAX-LDTEST) $150,150,180$

15? HOIMIN=APS(H(I,1)!

BO 17? $1=2, N$

IF I HIITMIN-ABS(H(I, I)I) $170,170,160$

15.) $H O I M I N=A B S(H I I, I)$

170 CONT INIUF

HDTEST $=$ HDIMIN $\$ R A P$

IF (HOTEST-XMAX) 1BC, 430,430

$180 N R=N R+1$

TANG=SIGNC 2.0, IHIIPIV, IPIVI-HIJPIV,JPIVI) I*HEIPIV,JPIVI/IABSIHIIPI IV, IPIV)-H(JPIV,JPIVI) +SOPT( (HIIPIV,IPIV)-HIJPIV,JPIVI) $\$ 2+4.0 * H(I D$ ZIV JPIV) $* 211$

COSINE $=1.0 / S O R T(1.0+T A N G * 2)$

SINE = IAINGRCOSINE

$H I I=H I I P I V, I P I V)$

HIIPIV,IPIV) $=$ COSI NE* $2 *(H I I+T A N G * 12.0 * H(I P I V, J P I V)+T A N G * H(J P I V$, JPI IVI)!

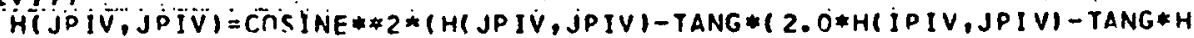
IIII!

$H(I P I V, J P I V)=0 . C$

IE (HIIDIV,IPIV)-H(JPIV, JPIV) $190,200,200$

190 HTEMP $=H($ I PIV, IPIVI

$H(I P I V, I P I V)=H(J P I V, J P I V)$ 
HI JPIV, JPI I I = HTF MP

HTR T:P=SION:(1.2, - SINE) \#C OSINE

$\operatorname{COS}[$ NTE = ABS (SINE)

SIN- =HTR ND

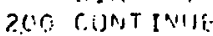

nu $\left.>7^{\circ} I=1, N !+1\right]$

IF (I-IPIV) $22: 2,70,210$

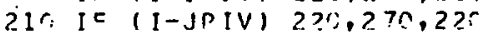

$2.2 n$ IF (IOII)-JPIV) $230,24(\cdot, 23 r$

$23 n$ IF $(I 0(I)-J P I V) \geq 7 \mathrm{C}, 24 \mathrm{C}, 270$

248 $K=I 0(i)$

$H T H M P=H(I, K)$

$H(I, K)=0 . C$.

$I P L I=I+1$

$\times(1)=? \cdot$ ?

DG $260 \mathrm{~J}=[\mathrm{PL}], \mathrm{N}$

IF: $(X(I)-\triangle P S(H(I, J))) 250,25 C, 260$

$250 \times(I)=\angle R S(H(I, J))$

$\operatorname{lo}(I)=J$

360 CONTINIJE

HII, KI I =HITEPP

290 CחIMTINU:

$x(1 P[\mathrm{P})=r .0$

$x(\mathrm{~T} F I V)=6.0$

กO $40, \quad I=1, N$

IF $(I-I P I V) 2 B C, 400, ? 28$

(B) HIK $M P=H(I$, IPIV $)$

$H(I, I N I V)=C O S I N E * H T E M P+S I N E * H(I, J P I V)$

IF (XII)-ARSIHII, IPIVI) $290,3 \cap 0,30 \mathrm{C}$

$290 \times(I)=A P S(H(I, I P I V))$ IO(1)=I?IV

$3 \cap \cap H(. I, J P I V)=-S ! N F: H T F M D+C O S I N E * H(I, J P I V I$ IF (XII)-ARSIHII, JPIVI) $310,400,40 \mathrm{C}$

$31 \cap X(1)=\triangle B S(H(1, J D I V))$

$\ln (!)=\mathrm{JP} ! \mathrm{V}$

rio to 400

320 IF $(1-J P I V) 331,400,360$

$3 ?$ ? HTFMP $=H(I P I V$, I) $H(I P[V, I)=C O S I N \&$ HTSMP+SINE*HI I, JPIVI IF (XIIPIV)-ABS(H(IDIV,I) $) 340,350,350$

$340 \times($ !PIV $)=\Delta R S(H(I P I V, I)$ IOIIIV) $=1$

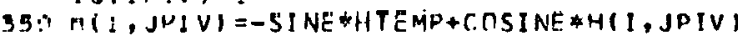
IF (XII)-APSIH(I,JPIVI) $310,400,4$ OOO

360 HTEMP $=$ HI I PIV, I ) H(IPIV,I) =CกS INF*HTEMP+SINE*HIJPIV, II IF (XIIPIVI-ABS(HIIPIV,I) $) 370,390,380$

$370 x(I \cap I V)=A C G(H(I P I V, I)$ Io(IPIV) $=1$

$3.8 \cap H(J P I V, I)=-S I N F$ \#HTEMP $+C$ CSINUE $\$ H\left(J^{n} I V, I\right)$ IF $(X(, I P I V)-\triangle R S(H(J P I V, I 1)) 390,400,400$

$390 \times(J P I V)=A B S(H(. I P I V+I))$ IO $($ I I IV IV $=I$

400 R.ONTINUF

IF (IIGFN) $90,416.90$

410 DO $420 \quad I=1,1$

$H T F M P=(I) I$, IPIV)

(II I, IPIV) =COSINE HTEMP+SINE*U(I,JPIV)

$42 n U(I, J P I V)=-S I N E$ \#TEMP+COSINE \#UII, JPIV) Go to yn

43? RETIIOH

B 59

B 6 r

B 61

R 62

B 63

A 64

B 65

B 66

A 67

B 68

B 69

B 70

B 71

ค 72

A 7.3

B 74

74
76

B 76

B 77

78

B 79

B 80

B 81

B 82

B 83

A 84

B 85

R 86

B 87

R 88

B 89

B 90

B 91

B 92

P 92

B 94

B 95

B 96

R 97

B 98

B 90

ค 100

B 101

B 122

B 103

a 104

D 105

B 106

B 107

B 108

B $1 \cap 9$

B 110

B 111

A 112

a 113

A 114

B 115

B 116

B 117

A 118

B 119

B $120-$ 


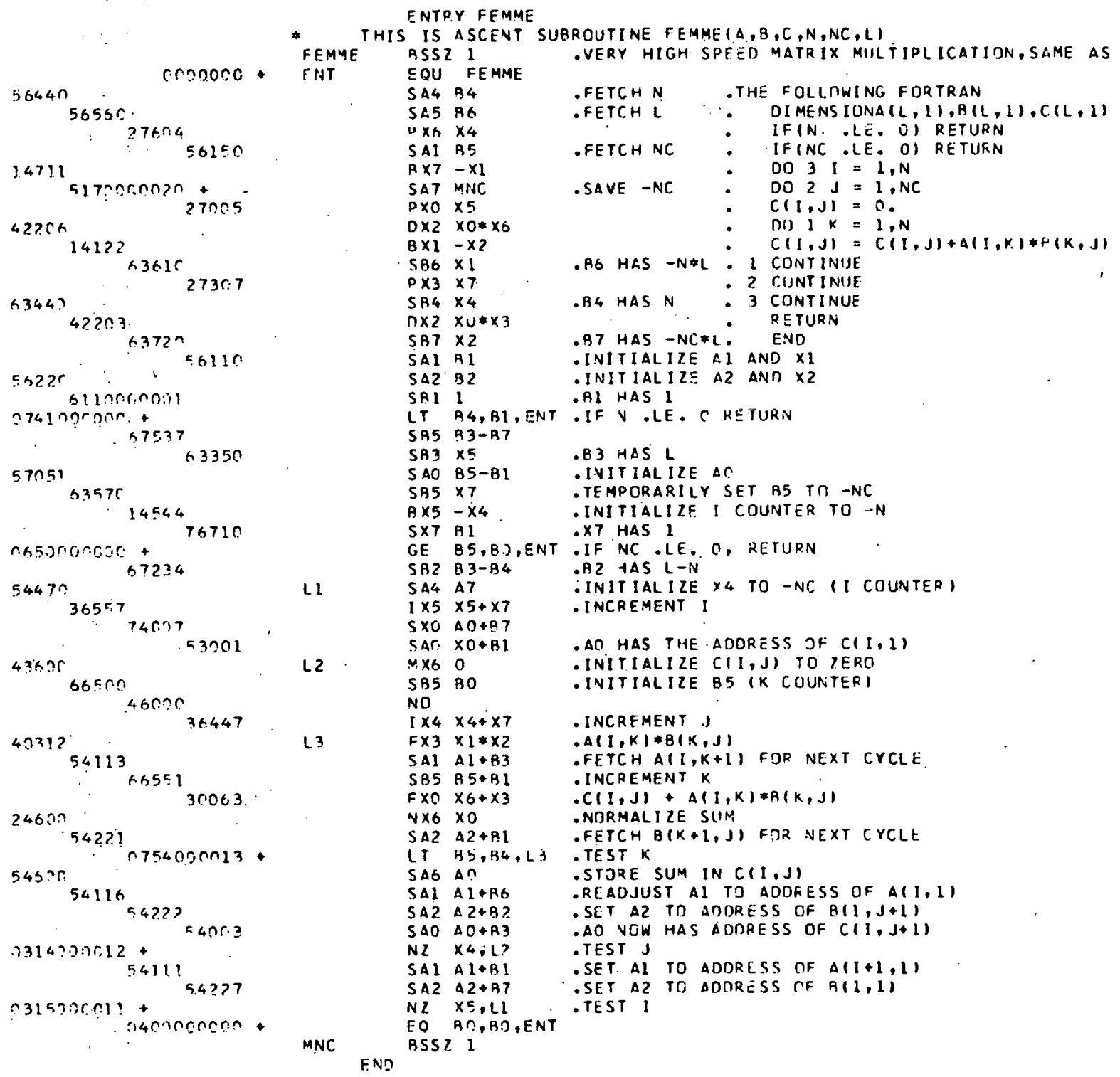




\section{References}

1. S. P. McGlynn, T. Azuml and M. Kinoshito, Molecular Spectroscopy of the Triplet State (Prentice Hall Inc., 1969).

2. R. S. Becker, Theory and Interpretation of Fluorescence and Phosphorescence (Wiley Interscience, inhn Wiley \&s Sons, Inc., 1769).

3. S. K. Lower and M. A. El-Sayed, Chem. Rev., 66, 199 (1966).

4. M. S. DeGroot, I. A. M. Hesselmann and J. H. van der Waals, Mol. Phys., 12, 259 (1967).

5. G. N. Lewis and M. J. Kasha, J. Am. Chem. Soc. 66, 2100 (1944).

6. G. N. Lewis and M. J. Kasha, J. Am. Chem. Soc. 67, 994 (1945).

7. G. N. Lewis and M. Calvin, T. Am. Chem. Soc. 67, 1832 (1945).

8. G. N. Lewis, M. J. Kasha, and M. Calvin, J. Chem. Phyo. 17, 804 (1949).

9. H. F. Hameka, thesis, Leiden (1956).

10. C. A. Hutchison and B. W. Mangum, J. Chem. Phys. 29, 952 (1958).

11. C. A. Hutehison and B. W. Mangram, J. Chem. Phys. 34, 908 (1961).

12. Ph. Kottis and R. Lefelivre, J. Chem. Phys. 39, 393 (1963).

13. Ph. Kottis and R. Lefelivre, J. Chem. Phys. 4I, 379 (1964).

14. S. Geschwind, G. E. DeVlin, R. L. Cohen, and S. R. Chinn, Phys. Rev. 137, A1087 (1965).

15. J. Brossel and A. Kastler, Compt. Rend. 229, 1213 (1949).

16. J. Brossel and F. Bitter, Phys. Rev. 86, 308 (1952). 
17. M. J. Scharnoff, J. Chem. Phys. 46, 3263 (1967).

18. A. L. Kwiram; Chem. Phys. Letters 1, 272. (1967).

19. J. Schmidt, I. A. M. Hesselmann, M. S. DeGroot and J. H. van der Waals, Chem. Phys. Letters 1 , 434 (1967).

20. J. Schmidt and J. H. van der Waals, Chem. Phys. Letters $\underline{2}, 640$ (1968).

21. R. W. Brandon, R. E. Gerkin and C. A. Hutchinson, Jr., J. Chem Phys. 41, 3717 (1968).

22. J. Schmidt and J. H. van der Waals, Chem Phys. Letters $\underline{3}, 546$ (1969).

23. D. S. Tinti, M. A. El-Sayed, A. H. Maki and C. B. Harris, Chem. Phyis. Letters $\underline{3}, 343$ (1969).

24. C. B. Harris, D. S. Tinti, M. A. El-Sayed and A. H. Maki, Chem. Phys. Letters 4 , 409 (1969).

25. I. Y. Chan, J. Schmidt and J. H. van der Waals, Chem. Phys. Letters 4, 269 (1969).

26. M. J. Buckley, C. B. Harris, and A. H. Maki, Chem. Phys. Letters 4, 591 (1970).

27. M. J. Buckley and C. B. Harris, Chem. Phys. Letters 5, 205 (1970).

28. T. S. Kuan, D. S. Tinti and M. A. El-Sayed, Chem. Phys. Letters $\underline{4}$, 507 (1970).

29. M. A. El-Sayed, D. S. Tinti and E. M. Yee, J. Chem. Phys. 51, 5721 (1969).

30. M. A. El-Sayed, D. S. Tinti and O. V. Owens, Chem. Phys. Letters $\underline{3}$, 339 (1969).

31. M. A. El-Sayed, J. Chem. Phys. 52, 6438 (1970). 
32. M. Schwoerer and H. C. Wolf, Molecular Crystals $\underline{3}, 177$ (1967).

33. M. A. El-Sayed and O. F. Kalman, J. Chem. Phys. 52, 4903 (1970).

34. M. Sharnoff, J. Chem. Phys. 51, 451 (1969).

35. M. Sharnoff, Symposium of the Faraday Society, No. 3, Magneto Optical Effects (1969).

36. D. Owens, M. A. El-Sayed and S. Ziegler, J. Chem. Phys. 52, 4315 (1970).

37. M. Sharnoff, Chem. Phys. Letters 2, 498 (1968).

38. L. Cheng and A. I. Kwiram, Chem. Phys. Trettiers 4 , 457 (1069).

39. J. Schmidt, W. S. Veeman and J. H. van der Waals, Chem. Phys. Letters 4, 341 (1969).

40. R. W. Brandon, R. E. Gerkin and C. A. Hutchison, Jr., J, Chem. Phys. 37. 447 (1962).

41. C. A. Hutchison, Jr., Phys. Soc. Japan. 17, Suppl. B-1, 458 (1962).

42. J. S. Vincent and A. H. Maki, J. Chem. Phys. 39, 3088 (1963).

43. J. S. Vincent and A. H. Maki, J. Chem. Phys. 42, 865 (1965).

44. O. H. Griffith, J. Phys. Chem. 69, 1429 (1965).

45. S. W. Charles, P. H. H. Fisher and C. A. McDowell, Mol. Phys. 2, 517 (1965).

46. R. W. Brandon, G. L. Closs and C. A. Hutchison, Jr., J. Chem. Phys. 37, 1878 (1962).

47. R. W. Brandon, G. L. Closs, C. E. Davoust, C. A. Hutchison, Jr., B. E. Kohler and R. Silbey, J. Chem. Phys. 43, 2006 (1965).

48. C. A. Hutchison, Jr., J. Phys. Chem. 71, 203 (1967). 
49. J. Ph. Grivet and J. M. Lhoste, Chem. Phys. Letters $\underline{3}, 445$ (1969).

50. J. S Vincent, J. Chem. Phys. 47, 1830 (1967).

51. W. S. Veeman and J. H. van der Waals, Chem. Phys. Letters 7, 65 (1970).

52. M. D. Fayer, D. A. Yuen and C. B. Harris, J. Chem Phys.,

53. C. B. Harris, J. Chem. Phys., in press.

54. D. Haarer and H. C. Wolf, Mol. Cryst. and Liq. Cryst. 10, 359 (1970).

55. J. Ph. Grivet, Chem.' Phys. Letters 4, 104.(1969).

56. H. F. Hameka in The Triplet State (proc. International Symposium on the Triplet State, 1967)(Cambridge University Press, 1967) p. 25.

57. A. Carrington and A. D. McLachlan, Introduction to Magnetic Resonance (Harper and Row, 1967) p. 117.

58. T. P. Das and E. L. Hahn, Nuclear Quadrupole Resonance Spectroscopy, (Academic Press, New York/London, 1958).

59. M. H. Cohen and F. Reif, Solid State Physics V.5 (Academic Press,

- New York/London, 1957).

60. C, P. Slichter, Principles of Magnetic Resonance (Harper and Row, New York, 1963).

61. A. Abragam, The Principles of Nuclear Magnetism, (Oxford University Press, 1961).

62. E. A. C. Lucken, Nuclear Quadrupole Coupling Constants (Academic Press, New York/London, 1969).

63. M. S. de Groot, I. A. M. Hesselmann, J. Schmidt and J. H. van der Waals, Mol. Phys. 15, 17. (1968). 
64. K. J. Standley and R. A. Vaughon, Electron Spin Relaxation Phenomena in Solids (Adam Hilger Ltd., London, 1969).

65. A. Abragam and B. Bleaney, Electron Paramagnetic Resonance of Transition Ions (Oxford University Press, 1970).

G6. M. A. El-Sayed, D. S. TInll and E. M. Yee, J. Chem. Phys. 5I, 5721 (1970).

67. M. A. El-Sayed, D. S. Tinti and D. V. Owens, Chem. Phys. Letters $\underline{3}$, 339 (1969).

68. E. Gilmore, G. Gibson and D. McClure, J. Chem. Phys. 20, 829 (1952); 23, 399. (1955).

69. D. S. McClure, J. Chem. Phys. 17, 665 (1949).

70. D. S. McClure, J. Chem. Phys. 17, 905 (1949).

71. G. Castro and R. M. Hochstrasser, J. Chem. Phys. 46, 3617 (1967).

72. R. H. Weul, Rev. 3il. Inslx. 33, 732 (1962).

73. M. Kasha, J. Opt. Sci. Am., 38, 929 (1948).

74. F. E. Terman, Electronic and Radio Engineering, (McGraw-Hill, 1955), p. 313 .

75. B. H. Smith, Lawrence Radiation Laboratory Engineering Note, EE-1187 (1967).

76. A. H. Francis and C. B. Harris, Chem. Phys. Letters, in press.

77. M. Tinkham and M. W. P. Strandberg, Phys. Rev. 97, 937 (1955).

78. M. S. de Groot, I. A. M. Hesselmann and J. H. van der Waals, Mol. Phys. 10, 91 (1965). 
79. S. L. Segal, R. G. Barnes and P. J. Bray, J. Chem. Phys. 25, 1286 (1956).

80. M. Dewar and E. Lucken, J. Chem. Soc. (London) 2653 (1958).

81. K. K. Innes, J. P. Byrne and I. G. Ross, J. Mol. Spectr. 22, 125 (1967):

82. L. Goodman and M. Kasha, J. Mol. Spectr. 2, 58 (1958).

83. J. E. Parkin and K. K. Innes, J. Mol. Spectr. 15, 407 (1965).

84. V. G. Krishna and I. Goodman, J. Chem. Phys. 36, 2217 (1962).

85. R. M. Hochstrasser and T. S. Iin, Symposium of the Farady Society, No. 3, Magneto Optical Effects (1969).

86. W. R. Moomaw and M. A. El-Sayed, J. Chem. Phys. 48, 2502 (1968).

87. H. Sternlicht, J. Chem. Phys. 38, 2316 (1963).

88. E. Schempp and P. J. Bray, J. Chem. Phys. 46, 1186 (1967).

89. P. B. Asycough, Electron Spin Resonance in Chemistry, Methuen and Co. Itd. London (1967).

90. M. A. El-Sayed, W. R. Moomaw and D. S. Tinti, J. Chem. Phys. 50, 1888 (1969).

91. J. Higuchi, J. Chem. Phys. 39, 1339 (1963).

92. E. Clementi, J. Chcm. Phys. 1.6, 1.737 (1967).

93. M. S. De Groot, I. A. M. Hesselman and J. H. van der Waals, Mol. Phys: 16, 45 (1969).

94. N. Hirota, C. A. Hutchison, Jr., and P. Palmer, J. Chem. Phys. 40, 3717 (1964). 
95. A. D. McLachlan, Mol. Phys. 5, 51 (1962).

96. C. L. Talcott and R. J. Myers, Mol. Phys. 12, 549 (1967).

97. G. A. George and G. C. Morris, Mol. Cryst. Liquid Cryst. 10, 187 (1970). 187. (1970).

98. R. R. Gilman and. J. De Heer, J. Chem. Phys. 52, 4287 (1969).

99. P. G. Russell and A. C. Albrecht, J. Chem. Phys. 4l, 2536 (1964).

100. M. D. Fáyer and C. B. Harris, Inorg. Chem. $\underline{8}, 2792$ (1969).

101. M. S. De Groot and J. H. van der Waals, Mol. Phys. 6, 545 (1963).

102. M. Godfrey, C. W. Kern and M. Karplus, J. Chem. Phys. 44,4459 (1966).

103. R. Bersohn, J. Chem. Phys. 22, 2078 (1954).

104. P. T. Rray, R. G. Bamco and R. Dersulu, J. Chem. Fhys. $25,8 \perp 3$ (19:60).

105. H. R. Falle, G. R. Iuckhurst, A. Horsfield and M. Ballester, J. Cher:1. Phys. 50, 2ל8 (1969).

106. D. Pooley and D. H. Whiffen, Spectrochim.Acta, 18, 291 (1962).

107. D. R. Kearns, J. Chem. Phys. 36, 1608 (1962).

108. A. C. Albrecht, J. Chem. Phys. 33, 157 (1960).

109. A. C. Albrecht, J. Chem. Phys. 38, 354 (1963).

110. C. Dean and R. V. Pound, J. Chem. Phys. 20, 195 (1952).

111. C. Dean, 'Ihesis, Harvard University (1952).

112. C. H. Townes, Handbuch der Physik, edited by S. Flügge (Springer Verlag, Berlin, 1958), Vol. 38, p. 444. 
113. M. Gouterman and W. Morfitt, J. Chem. Phys. 30, 1107 (1959).

114. M. Gouterman, J. Chem. Phys. 30, 1369 (1959).

115. H. Hamika, J. Chem. Phys. 31, 815 (1963).

116. Y. N. Chiu, J. Chem. Phys. 39, 2736 (1963).

117. A. Carrington and A. D. McLachlan, op.cit., p. 1:35-126.

118. H. Bayer, Z. Physik, 130, 227 (1951).

119. G. A. Jeffery and W. J. McVeagh, J. Chem. Phys. :3, 1165 (1955).

120. E. Frasson, C. Garbuguo and S. Bezzi, Acta Cryst. 12, 126 (1959).

121. A. H. Francis, Univ of Calif. (Berkeley), perso 1al communication (1970).

122. S. Saeki, Bull. Chem. Soc. Japan, 34, 1858 (1961).

123. M. Suzuki and M. Ito, Spectrochim. Acta, 25A, 1017 (1969).

124. C. A. Hutchison, Jr., J. V. Nicholas and G. W. Scott, J. Chem. Phys. 53, 1906 (1970). 
This report was prepared as an account of work sponsored by the United States Government. Neither the United States nor the United States Atomic Energy Commission, nor any of their employees, nor any of their contractors, subcontractors, or their employees, makes any warranty, express or implied, or assumes any legal liability or responsibility for the accuracy, completeness or usefulness of any information, apparatus, product or process disclosed, or represents that its use would not infringe privately owned rights. 
TECHNICAL INFORMATION DIVISION

LAWRENCE RADIATION LABORATORY UNIVERSITY OF CALIFORNIA

BERKELEY, CALIFORNIA 94720 